Fortschritt-Berichte VDl

VDI

Reihe 19

Wärmetechnik/

Kältetechnik

Nr. 162
Michael Schaub, M.Sc.

Berlin
Experimentelle

Betrachtung der

Wärmeübertragung

durch instationäre

freie Konvektion an

der vertikalen Platte 



\title{
Experimentelle Betrachtung der Wärmeübertragung durch instationäre freie Konvektion an der vertikalen Platte
}

\author{
vorgelegt von \\ Jan Michael Schaub, M.Sc. \\ geboren in Marburg \\ von der Fakultät III - Prozesswissenschaften \\ der Technischen Universität Berlin \\ zur Erlangung des akademischen Grades \\ Doktor der Ingenieurwissenschaften \\ - Dr.-Ing. - \\ genehmigte Dissertation
}

Promotionsausschuss:

Vorsitzender: Prof. Dr.-Ing. Felix Ziegler

Gutachter: $\quad$ Prof. Dr.-Ing. Martin Kriegel

Gutachter: Prof. Dr.-Ing. Clemens Felsmann

Tag der wissenschaftlichen Aussprache: 10.05.2019

Berlin, 2019 



\section{Fortschritt-Berichte VDI}

Reihe 19

Wärmetechnik/

Kältetechnik

Nr. 162
Michael Schaub, M.Sc.

Berlin

Experimentelle

Betrachtung der Wärmeübertragung durch instationäre freie Konvektion an der vertikalen Platte 


\title{
Schaub, Michael
}

\section{Experimentelle Betrachtung der Wärmeübertragung durch instationäre freie Konvektion an der vertikalen Platte}

\author{
Fortschr.-Ber. VDI Reihe 19 Nr. 162. Düsseldorf: VDI Verlag 2019. \\ 124 Seiten, 56 Bilder, 4 Tabellen. \\ ISBN 978-3-18-316219-2, ISSN 0178-9465, \\ $€$ 48,00/VDI-Mitgliederpreis € 43,20.
}

Für die Dokumentation: freie Konvektion - Wärmeübertragung - instationär - vertikale Platte - Luft - experimentell - analytisches Berechnungsverfahren

In der vorliegenden Dissertation werden die Auswirkungen von instationären Prozessen in freier Konvektion an der vertikalen Platte auf die Wärmeübertragung an Luft experimentell untersucht. Die dabei erhobenen Messwerte werden auf der Grundlage einer phänomenologischen Betrachtung in ein analytisches Berechnungsverfahren überführt. So legt eine physikalische Interpretation nahe, dass nach einer plötzlichen Veränderung der Wärmestromdichte ein Überschuss an potentieller Energie in der Strömungsgrenzschicht entsteht, der in einem anschließenden Ausgleichsvorgang durch zusätzliche Konvektionsstrukturen (insb. Kelvin-Helmholtz-Wirbell) in kinetische Energie umgewandelt wird. Betrachtet werden zyklische und sprungartige Variationen der Randbedingungen, deren praktische Anwendung erst durch die Etablierung der elektronischen Leistungsregelung relevant wurde. Derartige Betriebsweisen erlauben beispielsweise für die Wärmeübergabe von Raumheizsystemen eine Intensivierung der Übertragungsleistung bei gleicher mittlerer Oberflächentemperatur.

\section{Bibliographische Information der Deutschen Bibliothek}

Die Deutsche Bibliothek verzeichnet diese Publikation in der Deutschen Nationalbibliographie; detaillierte bibliographische Daten sind im Internet unter www.dnb.de abrufbar.

\section{Bibliographic information published by the Deutsche Bibliothek}

(German National Library)

The Deutsche Bibliothek lists this publication in the Deutsche Nationalbibliographie (German National Bibliography); detailed bibliographic data is available via Internet at www.dnb.de.

Alle Rechte, auch das des auszugsweisen Nachdruckes, der auszugsweisen oder vollständigen Wiedergabe (Fotokopie, Mikrokopie), der Speicherung in Datenverarbeitungsanlagen, im Internet und das der Übersetzung, vorbehalten. 


\section{Vorwort}

Die nachfolgende Dissertation entstand im Rahmen meiner Tätigkeit als wissenschaftlicher Mitarbeiter am Hermann-Rietschel-Institut, dem Fachgebiet Gebäude-Energie-Systeme der Technischen Universität Berlin.

In verschiedenen Forschungsprojekten des Instituts konnten die Potentiale einer instationären Betriebsweise von technischen Komponenten zur Steigerung der Energieeffizienz bei der Klimatisierung von Aufenthaltsräumen aufgezeigt werden. Die vorliegende Arbeit soll daher einen Beitrag zum Verständnis und zur Berechnung der damit verbundenen instationären Transportvorgänge leisten.

„Die Endlosigkeit des wissenschaftlichen Ringens sorgt unablässig dafür, dass dem forschenden Menschengeist seine beiden edelsten Antriebe erhalten bleiben und immer wieder von neuem angefacht werden: Die Begeisterung und die Ehrfurcht."

Max Planck, dt. Physiker (1858 - 1947)

Mein Dank für das entgegengebrachte Vertrauen, die inhaltlichen und methodischen Impulse, das fortdauernde Interesse und für ein stets offenes Ohr gilt dem Hauptgutachter Prof. Dr.-Ing. Martin Kriegel. Für die konstruktiven fachlichen Anregungen und die bereitwillige Übernahme der Mitbegutachtung danke ich Prof. Dr.-Ing. Clemens Felsmann. Außerdem danke ich meinen Kolleginnen und Kollegen am Institut für den fachlichen und freundschaftlichen Austausch, die begeisternde Arbeitsatmosphäre und für die Unterstützung bei der Durchführung der Experimente. Insbesondere danke ich jedoch meiner Familie und meinen Freunden für die fortdauernde Motivation, das Verständnis, die Unterstützung in vielerlei Hinsicht und für all das, was Ihr mir ermöglicht habt.

Berlin, im Frühjahr 2019

Michael Schaub 



\section{Inhalt}

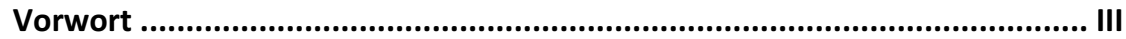

Nomenklatur............................................................................................ VIII

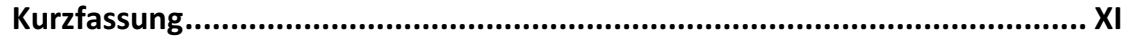

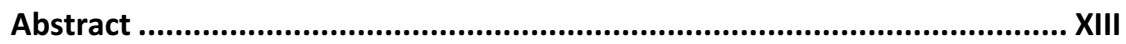

1 Freie Konvektion an der vertikalen Platte............................................... 1

1.1 Konvektive Wärmeübertragung ....................................................... 2

1.1.1 Räumlicher Bezug der Wärmeübertragungs-Intensität................... 5

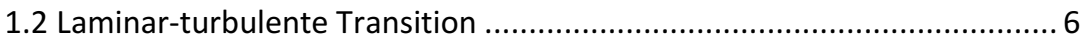

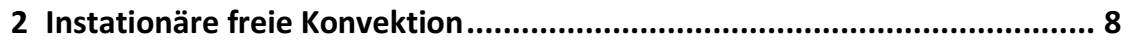

2.1 Beschreibung von instationären freien Konvektionsströmungen .............9

2.2 Berechnung von instationären Strömungsgrößen ............................... 11

2.2.1 Physikalische Vereinfachungen ............................................... 12

2.2.2 Modellbasierte Vereinfachungen ............................................. 14

2.3 Weiterführende Betrachtung der instationären Wärmeübertragung..... 17

3 Experimentelle Analyse der Wärmeübertragung durch freie Konvektion

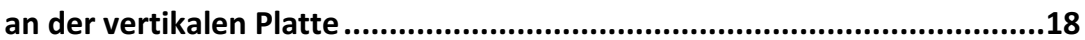

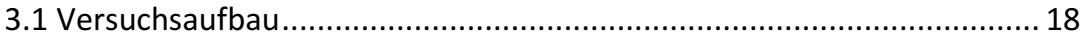

3.1.1 Konstruktion und Umgebungsbedingungen .............................. 19

3.1.2 Sandwich-Heizelemente ................................................................ 22

3.1.3 Leistungszufuhr und Regelung................................................. 24

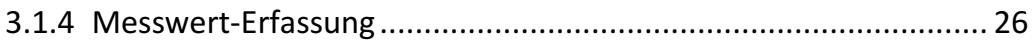

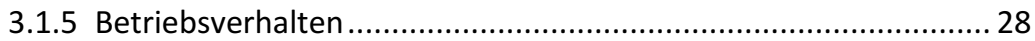

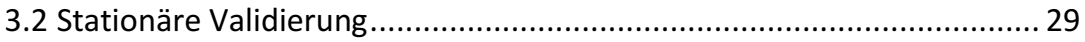

3.2.1 Bestimmung der Wärmeübertragung aufgrund von Strahlung..... 30

3.2.2 Bestimmung der konvektiven Wärmeübertragung...................... 30

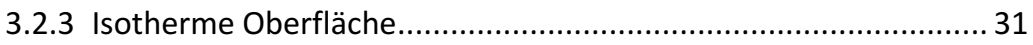

3.2.4 Homogene Wärmestromdichte ............................................... 33

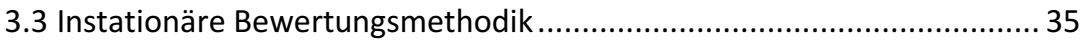

3.3.1 Quasi-stationärer Betrachtungsansatz..................................... 36

3.3.2 Bewertung der instationären Wärmeübertragung ....................... 38 
3.3.3 Thermische Kapazität der Sandwich-Heizelemente .................... 40

3.3.4 Messunsicherheits-Fortpflanzung …........................................ 42

3.4 Versuchsreihen mit veränderlichen Randbedingungen ......................... 42

3.4.1 Zyklische Variationen.............................................................. 42

3.4.2 Sprungartige Änderungen mit anschließender Beharrung............ 50

3.5 Schlussfolgerungen zur instationären Wärmeübertragung …................ 54

4 Phänomenologische Betrachtung ...........................................................55

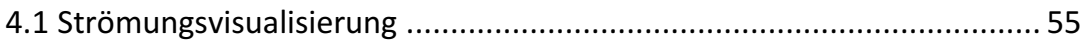

4.1.1 Versuchsaufbau zur Strömungsvisualisierung.............................. 55

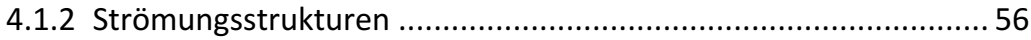

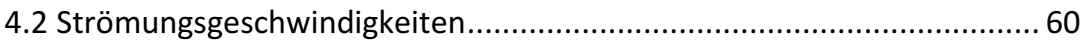

4.2.1 Versuchsaufbau zur Messung von Strömungsgeschwindigkeiten . 61

4.2.2 Geschwindigkeits-Fluktuationen .............................................. 62

4.3 Schlussfolgerungen zu instationären Strömungsstrukturen ....................65

5 Analytische Prognose der Wärmeübertragung durch instationäre

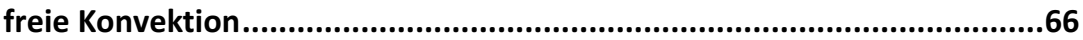

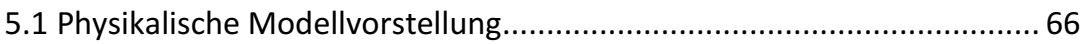

5.1.1 Potentielle und kinetische Energie der freien Konvektion .............66 66

5.1.2 Zeitliche Entwicklung der Wärmeübertragung durch

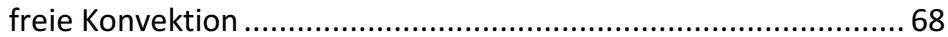

5.2 Prognosemodell zur instationären Wärmeübertragung bei

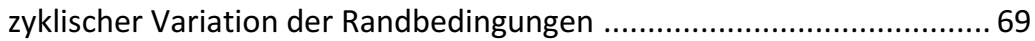

5.2.1 Validierungsmessungen für zyklische Variationen ........................ 70

5.2.2 Instationäre Phase bei zyklischen Variationen .............................. 73

5.2.3 Momentanwerte von zyklischen Variationen ............................. 76

5.2.4 Modellanwendung und Prognosegüte für zyklische Variationen.. 78

5.3 Prognosemodell zur instationären Wärmeübertragung bei

sprungartigen Änderungen mit anschließender Beharrung .................. 80

5.3.1 Validierungsmessungen für sprungartige Änderungen ................ 81

5.3.2 Instationäre Phase bei sprungartigen Änderungen........................ 83

5.3.3 Momentanwerte von sprungartigen Änderungen ........................ 85 
5.3.4 Modellanwendung und Prognosegüte für sprungartige Änderungen ...................................................... 87

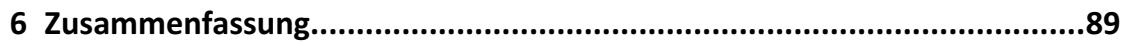

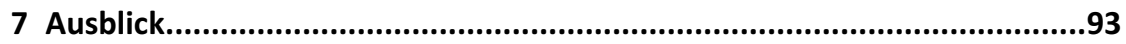

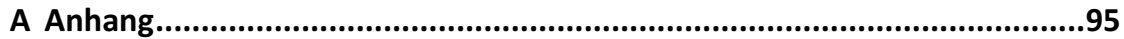

A.1 Ergebnisse der Validierungsmessungen für zyklische Variationen .......... 95

A.2 Ergebnisse der Validierungsmessungen für sprungartige Änderungen. 100

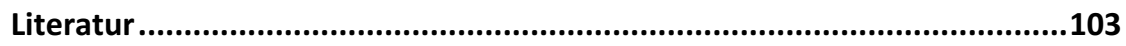




\section{Nomenklatur}

\begin{tabular}{|c|c|c|}
\hline Symbol & Einheit & Bedeutung \\
\hline \multicolumn{3}{|c|}{ Griechische Formelzeichen } \\
\hline$\alpha$ & $W /\left(m^{2} K\right)$ & Wärmeübergangskoeffizient \\
\hline$\beta$ & $1 / K$ & isobarer thermischer Volumen-Ausdehnungskoeffizient \\
\hline$\delta$ & $\mathrm{m}$ & Grenzschichtdicke \\
\hline$\Delta$ & - & Differenz \\
\hline$\varepsilon$ & - & Gesamtemissionsgrad \\
\hline$\vartheta$ & ${ }^{\circ} \mathrm{C}$ & Temperatur \\
\hline$\lambda$ & $\mathrm{W} /(\mathrm{m} \mathrm{K})$ & Wärmeleitfähigkeit \\
\hline$\mu$ & $\mathrm{kg} /(\mathrm{m} \mathrm{s})$ & dynamische Viskosität \\
\hline$v$ & $\mathrm{~m}^{2} / \mathrm{s}$ & kinematische Viskosität \\
\hline$\Pi$ & - & Verhältnis von Impulsgrößen zu mittleren Größen \\
\hline$\rho$ & $\mathrm{kg} / \mathrm{m}^{3}$ & Massendichte \\
\hline$\sigma$ & $\mathrm{Pa}$ & mechanische Spannung \\
\hline$\tau$ & s & Zeitkonstante \\
\hline \multicolumn{3}{|c|}{ Lateinische und sonstige Formelzeichen } \\
\hline$a$ & $\mathrm{~m}^{2} / \mathrm{s}$ & Temperaturleitfähigkeit \\
\hline$A$ & $m^{2}$ & Fläche \\
\hline$b$ & - & Regressionsparameter \\
\hline$c$ & $\mathrm{~J} /(\mathrm{kg} \mathrm{K})$ & spezifische Wärmekapazität \\
\hline C & $\mathrm{J} / \mathrm{K}$ & thermische Kapazität \\
\hline $\mathcal{D}$ & $\mathrm{kg} /\left(\mathrm{m} \mathrm{s}^{3}\right)$ & Diffusionsterm \\
\hline$e$ & $\mathrm{~J} / \mathrm{m}^{3}$ & spezifische Energie \\
\hline$f$ & $\mathrm{~N} / \mathrm{m}^{3}$ & volumenbezogene Kräfte \\
\hline$g$ & $\mathrm{~m} / \mathrm{s}^{2}$ & Schwerebeschleunigung \\
\hline$G r$ & - & Grashof-Zahl \\
\hline$H$ & $\mathrm{~J} / \mathrm{kg}$ & spezifische Totalenthalpie \\
\hline$L$ & $\mathrm{~m}$ & charakteristische Länge des Strömungsgebiets \\
\hline$m$ & - & Regressionsparameter \\
\hline$N u$ & - & Nußelt-Zahl \\
\hline
\end{tabular}




\begin{tabular}{|c|c|c|}
\hline Symbol & Einheit & Bedeutung \\
\hline$p$ & $\mathrm{~Pa}$ & Druck \\
\hline $\operatorname{Pr}$ & - & Prandtl-Zahl \\
\hline$\dot{q}$ & $\mathrm{~W} / \mathrm{m}^{2}$ & spezifische Leistung \\
\hline$R a$ & - & Rayleigh-Zahl \\
\hline $\operatorname{Re}$ & - & Reynolds-Zahl \\
\hline$s$ & - & Standardabweichung (Grundgesamtheit) \\
\hline$t$ & s & Zeit \\
\hline$T$ & $\mathrm{~K}$ & Temperatur \\
\hline$T u$ & $\%$ & Turbulenzgrad \\
\hline$u$ & $\mathrm{~m} / \mathrm{s}$ & Geschwindigkeitskomponente in x-Richtung \\
\hline$v$ & $\mathrm{~m} / \mathrm{s}$ & Geschwindigkeitskomponente in y-Richtung \\
\hline$V$ & $\mathrm{~m}^{3}$ & Volumen \\
\hline$\dot{V}$ & $\mathrm{~m}^{3} / \mathrm{h}$ & Volumenstrom \\
\hline$w$ & $\mathrm{~m} / \mathrm{s}$ & Geschwindigkeitskomponente in z-Richtung \\
\hline$x$ & - & Richtungskomponente (vertikal in Plattenebene) \\
\hline$y$ & - & Richtungskomponente (normal zur Plattenebene) \\
\hline$z$ & - & Richtungskomponente (horizontal in Plattenebene) \\
\hline \multicolumn{3}{|c|}{ Indices und Abkürzungen } \\
\hline * & & modifiziert (Randbedingung Wärmestromdichte) \\
\hline+ & & dimensionslos \\
\hline- & & zeitliches Mittel \\
\hline$\infty$ & & ungestörtes Umgebungsfluid \\
\hline 0 & & Referenzniveau der Stoffwerte \\
\hline $1 \tau$ & & Zeitraum für 63,2 \% der Sprungantwort \\
\hline c & & konvektiv \\
\hline char & & charakteristisch \\
\hline el & & elektrisch \\
\hline $\mathrm{F}$ & & Fluid \\
\hline is & & instationär \\
\hline is $\mathrm{Ph}$ & & instationäre Phase \\
\hline kin & & kinetisch \\
\hline
\end{tabular}




\begin{tabular}{ll}
\hline Symbol & Bedeutung \\
\hline L & geometrisches Mittel der charakteristischen Länge \\
lam & laminar \\
LSB & Bitwertigkeit (engl. least significant bit) \\
$\mathrm{m}$ & arithmetisches Mittel \\
$\max$ & Maximum \\
MB & Messbereich \\
min & Minimum \\
MW & Messwert \\
O & (Umgebungs-)Oberfläche \\
$\mathrm{p}$ & bei konstantem Druck \\
pot & potentiell \\
$\mathrm{r}$ & radiativ (Wärmestrahlung) \\
st & stationär \\
$\mathrm{t}$ & thermisch \\
turb & turbulent \\
$\mathrm{W}$ & (beheizte) Wandoberfläche \\
$\mathrm{x}$ & Ort auf Richtungskomponente x \\
$\mathrm{y}$ & Ort auf Richtungskomponente y \\
$\mathrm{z}$ & Ort auf Richtungskomponente z \\
\hline
\end{tabular}




\section{Kurzfassung}

Die vorliegende Ausarbeitung untersucht den Einfluss von instationären Strömungsstrukturen in freier Konvektion auf die Wärmeübertragung von einer vertikalen Platte an Luft. Dazu werden die mittlere und die momentane Intensität der konvektiven Wärmeübertragung für zyklische und sprungartig variierende Betriebsweisen einer direkt-elektrisch beheizten, 2 m hohen Kupferplatte experimentell erfasst.

Bei Dauerbetriebstemperaturen von maximal $100{ }^{\circ} \mathrm{C}$ und Impuls-Wärmestromdichten von bis zu $1.430 \mathrm{~W} / \mathrm{m}^{2}$ werden sowohl laminare, als auch transiente und turbulente Strömungsformen betrachtet. Im Vergleich zur einer quasi-stationären Betrachtungsweise tritt im instationären Fall eine mittlere Intensivierung der konvektiven Wärmeübertragung von maximal $23,8 \%$ bei zyklischen Variationen der Randbedingungen und von bis zu 30,2 \% bei sprungartigen Änderungen auf. Die stärkste Beeinflussung der konvektiven Wärmeübertragung durch eine instationäre Betriebsweise zeigt sich im Bereich der kritischen Grashof-Zahl, also zu Beginn der laminar-turbulenten Transition. Hinsichtlich der zeitlichen Entwicklung der Wärmeübertragungs-Intensität treten die größten Überschreitungen des quasi-stationären Vergleichsniveaus stets zum Ende eines Wärmestrom-Impulses auf.

Phänomenologische Betrachtungen der instationären Konvektionsströmung in Form von Visualisierungen zeigen bei einer impulsartigen Wärmestromzufuhr eine temporär verstärkte Durchmischung der Grenzschicht. Exemplarische Messungen zur zeitlichen Entwicklung der Strömungsgeschwindigkeiten innerhalb der Grenzschicht zeigen dabei ebenfalls stark ausgeprägte irreguläre Fluktuationen. Die phänomenologischen Beobachtungen legen daher nahe, dass überschüssige potentielle Energie, die durch einen hinreichend intensiven Wärmestrom-Impuls plötzlich in eine Auftriebsströmung eingebracht wird, zunächst durch das temporäre Aufkommen von großskaligen Rotationsbewegungen in kinetische Energie umgewandelt wird, bevor sich eine quasi-stationäre Strömungsentwicklung einstellt.

Die Grenzschichtfluktuationen stehen darüber hinaus in guter zeitlicher Übereinstimmung mit der instationären Phase der Wärmeübertragung. Die vorübergehende Intensivierung des konvektiven Wärmetransports wird dement- 
sprechend auf eine kurzzeitig verstärkte Durchmischung der Grenzschicht zurückgeführt.

Auf Grundlage der experimentellen Ergebnisse, für die eine sehr gute quantitative und qualitative Reproduzierbarkeit vorliegt, wird ein analytisches Berechnungsmodell vorgestellt. Als zentrale Einflussgrößen werden darin die mittlere Grashof-Zahl und eine dimensionslose Kennzahl $\Pi$ berücksichtigt, welche als Maß für den mittleren Überschuss der potentiellen Energie innerhalb der instationären Phase interpretiert wird.

Das Berechnungsmodell erlaubt sowohl die Prognose der mittleren, als auch der momentanen Wärmeübertragungs-Intensität durch instationäre freie Konvektionsströmungen an der vertikalen Platte. So eröffnet sich für derartige Wärmeübertrager durch eine gezielte Variation der Betriebsweise die Möglichkeit zu einer Intensivierung der Übertragungsleistung bei gleichen mittleren Oberflächentemperaturen oder zu einer Reduzierung der mittleren Oberflächentemperaturen bei gleicher Übertragungsleistung. 


\section{Abstract}

The present elaboration investigates the influence of unsteady flow structures in natural convection on the heat transfer from a vertical flat plate to air. Therefore, the mean and momentary intensity of convective heat transfer are determined experimentally for cyclic and step-like changes in the operation mode of a direct-electrically heated, $2 \mathrm{~m}$ high copper plate.

At continuous operating temperatures of max. $100{ }^{\circ} \mathrm{C}$ and impulse heat flux densities of up to $1,430 \mathrm{~W} / \mathrm{m}^{2}$, laminar, transitional and turbulent flows are considered. Compared to a quasi-stationary approach, in the unsteady case, a maximum increase in the mean convective heat transfer of $23.8 \%$ occurs for cyclic variations of the boundary conditions and of up to $30.2 \%$ for step-like changes. The strongest influence of an unsteady operation mode on the convective heat transfer occurs in the range of the critical Grashof number - i.e. at the beginning of the laminar-turbulent transition. In terms of the temporal development of the heat transfer intensity, the largest exceedances of the quasistationary comparison level consistently occur at the end of a heat flux impulse.

Phenomenological observations of the unsteady convection flow in the form of visualizations show a temporarily increased mixing of the boundary layer in the case of an impulse-like heat flux supply. Exemplary measurements of the temporal development of the flow velocities within the boundary layer also show very distinctive irregular fluctuations. Thus, the phenomenological observations indicate that surplus potential energy, which is suddenly transferred into a buoyant flow by a sufficiently intensive heat flux impulse, is initially converted into kinetic energy through the temporary appearance of large-scale rotational structures, before a quasi-stationary flow development occurs.

Furthermore, the boundary layer fluctuations are in good temporal agreement with the unsteady phase of heat transfer. The temporary enhancement of the convective heat transport is accordingly attributed to a short-time intensified mixing of the boundary layer.

Based on the experimental results, for which a very good quantitative and qualitative reproducibility prevails, an analytical calculation model is presented. The mean Grashof number and a dimensionless parameter $\Pi$ (which is interpreted 
as a measure of the mean potential energy surplus within the unsteady phase) are considered as main influencing quantities.

The calculation model allows the prediction of the mean as well as the momentary heat transfer intensity due to unsteady natural convection flows at the vertical plate. Consequently, for these type of heat exchangers, an unsteady operation mode allows an increase in the heat transfer intensity for the same average surface temperatures or a decrease in the average surface temperatures for the same heat transmission rate. 


\section{Freie Konvektion an der vertikalen Platte}

In zahlreichen natürlichen und technischen Prozessen spielen freie (oder natürliche) Konvektionsströmungen eine signifikante Rolle für den Transport von Wärme. Ein für technische Prozesse besonders relevantes Beispiel für einen derartigen, durch Gravitation und Dichteunterschiede induzierten Molekültransport sind Auftriebs- oder Fallströmungen von Fluiden in der Nähe von Festkörpern mit abweichender Temperatur.

Der Transport von Wärme zwischen Festkörper und Fluid geschieht dabei innerhalb der Grenzschicht, in der die Strömungsgeschwindigkeit $u$ des Fluids vom Wert Null an der Festkörperwand (Haftbedingung) über ein Maximum bis zum Ruhezustand des Umgebungsfluids variiert, wie in Abbildung 1.1 für den Fall einer isothermen Wand dargestellt.

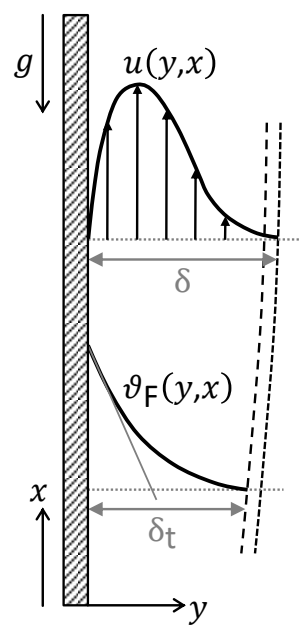

Abbildung 1.1: Grenzschichtstruktur bei freier Konvektion an der isothermen vertikalen Platte

Da der Wärmetransport bei einer Fluidgeschwindigkeit von Null in unmittelbarer Wandnähe ausschließlich aufgrund von Wärmeleitung erfolgt, charakterisiert der Temperaturgradient des Fluids $\partial \vartheta_{\mathrm{F}} / \partial y$ an der Wand gemäß Gleichung 1 (Fourier'sches Wärmeleitungsgesetz) zugleich die Intensität der kon- 
vektiven Wärmeübertragung $\dot{q}_{c}$, sofern Wärmestrahlung unberücksichtigt bleibt.

$$
\dot{q}_{\mathrm{c}}=-\lambda_{\mathrm{W}} \cdot\left(\frac{\partial \vartheta_{\mathrm{F}}}{\partial y}\right)_{\mathrm{y}=0}
$$

Bei freien Konvektionsströmungen an der vertikalen Platte weist die Strömungsgrenzschicht $\delta$ stets eine größere Dicke im Vergleich zur Temperaturgrenzschicht $\delta_{\mathrm{t}}$ auf, da am Grenzschichtrand auch temperatur- und somit dichteneutrale Teilchen des Umgebungsfluids eine Impulsübertragung von der Auftriebs- oder Fallströmung erfahren.

\subsection{Konvektive Wärmeübertragung}

Eine analytische Berechnung der Temperatur- und Geschwindigkeitsprofile gelingt bislang aufgrund der Kopplung von Energie- und Impulstransport nicht (vgl. Kapitel 2.2.1). Die Wärmeübertragung durch Transmission in unmittelbarer Wandnähe und der konvektive Molekültransport in der Grenzschicht werden daher bevorzugt in einem konvektiven Wärmeübergangskoeffizienten $\alpha_{\mathrm{c}}$ zusammengefasst (Gleichung 2).

$$
\alpha_{\mathrm{c}}=\frac{\dot{q}_{\mathrm{c}}}{\vartheta_{\mathrm{w}}-\vartheta_{\infty}}
$$

Der somit beschriebene Zusammenhang von konvektiver Wärmestromdichte $\dot{q}_{\mathrm{c}}$ und der vorliegenden Temperaturdifferenz zwischen Oberfläche und dem ungestörten Umgebungsfluid $\Delta \vartheta_{\mathrm{W}-\infty}$ wird insbesondere zur Quantifizierung der Wärmeübertragungs-Intensität einer konkreten technischen Anwendung herangezogen.

Zur Erarbeitung von Korrelationen, deren Gültigkeit nicht nur auf ein individuelles Problem oder ein willkürlich gewähltes Maßsystem beschränkt bleibt, werden darüber hinaus dimensionslose Kennzahlen gebildet. Im Falle des konvektiven Wärmeübergangskoeffizienten $\alpha_{\mathrm{c}}$ gelingt dies mit Hilfe einer charakteristischen Länge $L$ und der Wärmeleitfähigkeit des Umgebungsfluids $\lambda_{F}$ (Gleichung 3). 


$$
N u_{\mathrm{L}}=\frac{\alpha_{\mathrm{c}} \cdot L}{\lambda_{\mathrm{F}}}
$$

Die dabei resultierende $\mathrm{Nußelt-Zahl} \mathrm{Nu}$ kann folglich als dimensionslose Intensität der konvektiven Wärmeübertragung interpretiert werden.

Kennzeichnend für die Intensität von freien Konvektionsströmungen sind neben den Auftriebskräften aus temperaturbedingten Dichteunterschieden auch die entgegenwirkenden Reibungskräfte aufgrund von Zähigkeit sowie die Trägheitskräfte. Ausgehend von einer linearen Kopplung zwischen Fluidtemperatur und -dichte sowie unter Berücksichtigung von Dichteunterschieden ausschließlich im Auftriebsterm (Oberbeck-Boussinesq-Approximation, vgl. Kapitel 2.2.1) kann der Zusammenhang von Auftriebs-, Zähigkeits- und Trägheitskräften mit der dimensionslosen Grashof-Zahl Gr gemäß Gleichung 4 beschrieben werden.

$$
G r_{\mathrm{L}}=\frac{g \cdot \beta_{\infty} \cdot\left(\vartheta_{\mathrm{W}}-\vartheta_{\infty}\right) \cdot L^{3}}{v^{2}}
$$

Für den Fall einer homogenen Wärmestromdichte anstelle einer isothermen Oberfläche kann die Grashof-Zahl auch in eine modifizierte Form $G r^{*}$ überführt werden (Gleichung 5).

$$
G r_{\mathrm{L}}^{*}=G r_{\mathrm{L}} \cdot N u_{\mathrm{L}}=\frac{g \cdot \beta_{\infty} \cdot \dot{q}_{\mathrm{c}} \cdot L^{4}}{v^{2} \cdot \lambda_{\mathrm{F}}}
$$

Das Verhältnis von Impuls- und Wärmetransport in einer Grenzschicht wird durch zustandsabhängige Stoffparameter mit Hilfe der Prandtl-Zahl Pr nach Gleichung 6 wiedergegeben.

$$
\operatorname{Pr}=\frac{v}{a}
$$

Durch eine Kopplung der Kräfteverhältnisse mit den fluidspezifischen Transporteigenschaften gemäß Gleichung 7 resultiert die ebenfalls dimensionslose Rayleigh-Zahl Ra.

$$
R a_{\mathrm{L}}=G r_{\mathrm{L}} \cdot \operatorname{Pr}
$$

Sie wird bei freier Konvektion häufig für eine fluidunabhängige Charakterisierung der Strömungsform herangezogen, soll also u.a. einen Rückschluss darauf 
geben, ob eine Strömung geordnet-laminare oder chaotisch-turbulente Strukturen aufweist.

Tatsächlich weist die laminar-turbulente Transition jedoch eine Abhängigkeit vom Impuls- und Wärmetransport innerhalb eines jeweiligen Fluids, also von dessen Prandtl-Zahl, auf (vgl. [Bejan und Lage 1990] sowie [Vitharana und Lykodis 1994]). Im Folgenden wird daher die fluidspezifische Grashof-Zahl als Stabilitäts-Kriterium herangezogen. Für das Beispiel der freien Konvektion von Luft an der vertikalen, isothermen Platte vollzieht sich die Transition in einem Bereich von ca. $10^{9}<G r_{L}<2 \cdot 10^{10}$ (vgl. z.B. [Pirovano et al 1970], [Siebers et al. 1985] sowie [Tsuji und Nagano 1989]). Bei einer homogenen Wärmestromdichte liegt der Transitionsbereich bei $10^{11}<G r_{\mathrm{L}}^{*}<5 \cdot 10^{12}$ (vgl. [Miyamoto und Okayama 1982] sowie [Kitamura et al. 1985]).

Die dargestellten Kennzahlen der Ähnlichkeitstheorie erlauben es schließlich, die Intensität der konvektiven Wärmeübertragung auf der Basis von empirischen Korrelationen abzuschätzen. Im Bereich der freien Konvektion kommt dabei den Korrelationen von [Churchill und Chu 1975] in den Gleichungen 8 und 9 die größte Relevanz zu. Sie basieren auf Messdaten zahlreicher Autoren in einem sehr weiten Anwendungsbereich (ca. $10^{-1}<R a_{\mathrm{L}}<10^{12}$ ) und sind bei isothermer Oberfläche mit einer Gültigkeit für Fluide beliebiger Prandtl-Zahl angegeben.

$$
\begin{aligned}
& 0<R a_{\mathrm{L}}<10^{9}: \\
& N u_{\mathrm{L}}=0,68+\frac{0,67 \cdot R a_{\mathrm{L}}^{1 / 4}}{\left(1+\left(\frac{0,492}{P r}\right)^{9 / 16}\right)^{4 / 9}}
\end{aligned}
$$

$$
R a_{\mathrm{L}}>10^{9}:
$$

$$
N u_{\mathrm{L}}=\left(0,825+\frac{0,387 \cdot R a_{\mathrm{L}}^{1 / 6}}{\left(1+\left(\frac{0,492}{P r}\right)^{9 / 16}\right)^{8 / 27}}\right)^{2}
$$

Für den Fall einer homogenen Wärmestromdichte kann der Faktor 0,492 durch den Wert 0,437 ersetzt werden. $R a_{\mathrm{L}}$ ist dann auf Grundlage der Gleichungen 5 und 7 iterativ mit Hilfe von Gleichung 10 zu bestimmen. 


$$
R a_{\mathrm{L}}=R a_{\mathrm{L}}^{*} / N u_{\mathrm{L}}
$$

Eine Übersicht der bekannten Korrelationen für verschiedene Randbedingungen wird beispielsweise in [Khalifa 2001], [Aydin und Guessous 2001] sowie [Martynenko und Khramtsov 2005] gegeben. Die Intensität der konvektiven Wärmeübertragung wird dabei vorwiegend als Potenzfunktion in Abhängigkeit der Grashof- und Prandtl-Zahl approximiert. Für die isotherme vertikale Platte werden Exponenten in der Größenordnung von $1 / 5$ bis $1 / 4$ im laminaren und ca. $1 / 3$ bis $2 / 5$ im turbulenten Bereich angegeben (vgl. hierzu auch [Martin 1984]). Bei homogener Wärmestromdichte verringern sich die Exponenten auf zumeist 1/5 für laminare und etwa 1/4 für turbulente Strömungen.

\subsubsection{Räumlicher Bezug der Wärmeübertragungs-Intensität}

Für die dargestellte Beschreibung der stationären WärmeübertragungsIntensität durch freie Konvektionsströmungen anhand der Nußelt-Zahl in Abhängigkeit der Grashof-Zahl ist jedoch eine Unterscheidung zwischen globalen und lokalen Angaben zu beachten. Aus semi-empirischen KorrelationsGleichungen, wie z.B. den Gleichungen 8 und 9, gehen stets mittlere globale Nußelt-Zahlen für das gesamte Strömungsgebiet mit der charakteristischen Länge $L$ hervor. Für lokale Werte werden in der Literatur hingegen unterschiedliche Bilanzräume verwendet. So beziehen sich einige Autoren (vgl. z.B. [Pirovano et al. 1970] sowie [Miyamoto und Okayama 1982]) bei der Angabe von lokalen Nußelt-Zahlen tatsächlich auf die Intensität der Wärmeübertragung an einem bestimmten Ort $x$ innerhalb des Strömungsgebiets $L$. Davon abweichend ist auch ein Bezug der Wärmeübertragungs-Intensität auf ein Teilgebiet der betrachteten Strömung etabliert (vgl. z.B. [Warner und Arpaci 1968]). Die lokalen Nußelt-Zahlen korrespondieren dann mit einer Bilanzierung vom Beginn der Strömung (leading-edge, $x=0$ ) bis zu einem Ort $x / L$ (siehe auch Abbildung 3.6 in Kapitel 3.1.2). Dieser Vorgehensweise liegt die Annahme zugrunde, dass stromabwärts befindliche Bereiche keine Rückwirkungen auf das betrachtete Teilgebiet der Strömung haben.

Aus zahlreichen Quellen geht die Bilanzierungsart für lokale Angaben nicht eindeutig hervor (vgl. [Yang 2001] mit Bezug auf [Saunders 1936], [Cheeswright 1968], [Vliet und Ross 1975], [Kitamura et al. 1985], [Siebers et al. 1985], [Tsuji 
und Nagano 1988] und [Tsuji und Nagano 1989] sowie [Hattori et al. 2006]). Zumeist ist jedoch eine Zuordnung anhand des Verlaufs der einzelnen Datenpunkte möglich. So wird der Übergangsbereich von der laminaren zur turbulenten Strömungsform insbesondere bei einer Angabe der Nußelt-Zahlen von Einzelpositionen $N u_{\mathrm{x}}$ deutlich (siehe z.B. Abbildung 3.11 in Kapitel 3.2.3). Bei Bilanzierung über einen Teilbereich des Strömungsgebiets $x / L$ resultiert letztlich ein quasi-globaler Wert $N u_{\mathrm{x} / L}$, der ggf. laminare, transiente und turbulente Strömungsformen beinhaltet. In doppelt-logarithmischer Darstellung verstetigt sich in der Folge die Anordnung der Datenpunkte im Transitionsbereich (siehe u.a. Abbildung 3.12 in Kapitel 3.2.3).

Um eine eindeutige Unterscheidung der jeweils bilanzierten Strömungsbereiche zu ermöglichen, werden im Folgenden die Indices $L$ für globale Bilanzen des gesamten Strömungsgebiets, $x / L$ für quasi-globale Angaben zu einem Teilbereich und $x$ für lokale Einzelwerte eines Ortes verwendet.

\subsection{Laminar-turbulente Transition}

Der Transitions-Prozess einer stationären freien Konvektionsströmung an der vertikalen Platte ist in Abbildung 1.2 schematisch sowie interferometrisch dargestellt.

Grenzt ein ruhendes Fluid an eine beheizte vertikale Oberfläche an, so stellen sich aufgrund der in Gleichung 1 beschriebenen Wärmeleitung Temperaturund somit Dichteunterschiede im Fluid ein. Beginnend am unteren Ende der Platte setzt eine zunächst laminare Auftriebsströmung mit geordneten Stromlinien ein (leading-edge-effect). Die resultierenden Geschwindigkeitsunterschiede zwischen Strömungsgrenzschicht und ruhendem Umgebungsfluid lassen lokale Trennschichten entstehen. Dort auftretende Scherkräfte stören im weiteren Verlauf die laminare Stabilität, weshalb sich vermehrt zweidimensionale Strukturen, insbesondere Tollmien-Schlichting-Wellen, ausbilden. Mit zunehmender Strömungsweglänge werden diese durch dreidimensionale, $\Lambda$-förmige Wellen überlagert, die in Turbulenzflecken zerfallen und schließlich in eine vollturbulente Strömungsform übergehen.

In turbulenten Strömungsgebieten tritt eine verstärkte Durchmischung der Grenzschicht mit weiter entferntem Umgebungsfluid auf, was u.a. zu einer er- 
heblichen Intensivierung der Wärmeübertragung zwischen Oberfläche und Fluid führt. Ein Ansatz zur Beschreibung dieses makroskopischen konvektiven Impuls- und Energietransports besteht in Analogie zur molekularen Viskosität und Temperaturleitfähigkeit in der Einführung turbulenter Transportgrößen (siehe Kapitel 2.2.2).
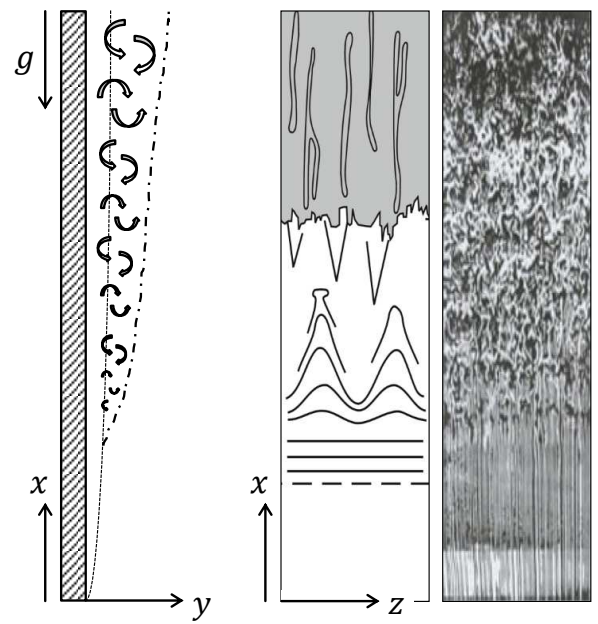

Abbildung 1.2: Laminar-turbulente Transition bei freier Konvektion an der vertikalen Platte

(Bildquelle: bearbeitet nach [Oertel jr. 2017]) 


\section{Instationäre freie Konvektion}

Insbesondere in turbulenten Strömungen müssen die Temperatur- und Geschwindigkeitswerte an jedem Ort einer Grenzschicht als zeitlich fluktuierend betrachtet werden. Die in den Kapiteln 1.1 und 1.2 dargestellten Zusammenhänge, welche für einen vollständig ausgebildeten Strömungszustand gelten, beziehen sich somit auf zeitlich konstante Mittelwerte der Schwankungsgrößen (im Folgenden: statistisch stationär). Während der Entstehungs- oder Abklingphase einer Strömung sowie bei einer gezielten Variation der Randbedingungen muss jedoch die gesamte Strömung als zeitvariabel angesehen werden (im Folgenden: instationär).

Die Intensität von Geschwindigkeits-Fluktuationen, die an einem bestimmten Ort $x$ innerhalb der Strömungsgrenzschicht auftreten, kann mit Hilfe des Turbulenzgrades $T u_{\mathrm{x}}$ gemäß Gleichung 11 quantifiziert werden.

$$
T u_{\mathrm{x}}=s\left(u_{\mathrm{x}}^{+}\right) / \bar{u}_{\mathrm{x}}^{+}
$$

Die darin verwendete dimensionsfreie Geschwindigkeit $u_{\mathrm{x}}^{+}$resultiert gemäß Gleichung 12 aus einem Bezug der dimensionsbehafteten Grenzschichtgeschwindigkeit $u_{\mathrm{x}}$ auf die charakteristische Geschwindigkeit der freien Konvektionsströmung $u_{\text {char }}$. Deren Bestimmungsgleichung 13 geht aus einer Gleichsetzung der Grashof-Zahl mit dem Quadrat der Reynolds-Zahl hervor (vgl. z.B. [Oertel jr. 2017]).

$$
\begin{aligned}
& u_{\mathrm{x}}^{+}=u_{\mathrm{x}} / u_{\text {char }} \\
& u_{\text {char }}=\sqrt{g \cdot x / L \cdot \beta_{\infty} \cdot \Delta \vartheta_{\mathrm{W}-\infty}}
\end{aligned}
$$

Weist der so bestimmte Turbulenzgrad im zeitlichen Verlauf Veränderungen auf, wird die Strömung am betrachteten Ort der Grenzschicht als instationär interpretiert (siehe Kapitel 4.2). 


\subsection{Beschreibung von instationären freien Konvektions- strömungen}

In diesem Sinne instationäre freie Konvektionsströmungen wurden v.a. hinsichtlich des laminaren Anlaufverhaltens eines anfänglich ruhenden Fluids ausführlich betrachtet. Zahlreiche theoretische (vgl. z.B. [Gebhart 1961] und [Gebhart 1963]) sowie experimentelle (vgl. z.B. [Gebhart und Adams 1963], [Sammakia et al. 1985] und [Maranzana et al. 2002]) Untersuchungen hierzu zeigen hinreichend genaue Berechnungsergebnisse, wenn für die jeweils aktuellen Randbedingungen eines Zeitpunktes die Berechnungs-Korrelationen für statistisch stationäre Strömungen herangezogen werden (im Folgenden: quasistationär). Voraussetzung ist, dass sich die Temperaturänderung an der Oberfläche langsamer vollzieht, als die Ausbildung der Grenzschicht (vgl. [Saha et al. 2012]). Zusammenfassende zeitliche Skalierungs-Korrelationen für die Grenzschichtdicken, Strömungsgeschwindigkeiten und Wärmeübergänge von laminaren Strömungen bei sinusförmigen Temperaturschwankungen geben [Lin und Armfield 2017] an. Für sehr schnelle Temperaturänderungen an der wärmeübertragenden Oberfläche wurden in der Grenzschicht von laminaren Strömungen hingegen auch Fluktuationen der Temperaturen (vgl. [Patterson et al. 2002] und [Zhao et al. 2016]) und Geschwindigkeiten (vgl. [Zhao et al. 2019]) beobachtet, die deutlich von einem quasi-stationärem Verlauf abweichen. Im Bereich der (teil-)turbulenten instationären freien Konvektionsströmungen befasst sich ein Großteil der bisherigen Betrachtungen mit der phänomenologischen und analytischen Beschreibung von Störfrequenzen, die einer statistisch stationären Strömung (teilweise zeitvariabel) aufgeprägt werden. Sowohl experimentelle, als auch numerische Untersuchungen kommen zu dem Schluss, dass bestimmte Störfrequenzen u.a. in Abhängigkeit der Grashof-Zahl entweder verstärkend oder dämpfend wirken (vgl. z.B. [Gebhart 1969], [Mollendorf und Gebhart 1970], [Cheeswright und Doan 1978] sowie [Paul und Rees 2008]). Einige Arbeiten interpretieren dies als eine Art Filter-Wirkung der Grenzschicht. Demnach sei vor allem bei den Störfrequenzen eine Anfachung der Turbulenz festzustellen, die der charakteristischen Eigenfrequenz der Temperaturgrenzschicht entsprechen (vgl. [Joshi und Gebhart 1987] sowie [Zhao et al. 2013]). 
Neben Strömungs-Beeinflussungen durch extern aufgeprägte thermische oder mechanische Störungen konnten einige Untersuchungen auch während des Aufheiz- und Abkühlvorgangs von beheizten vertikalen Oberflächen instationäre Entwicklungen in turbulenten Strömungen nachweisen. So gelang es beispielsweise [Joshi und Gebhart 1987] sowie [Joshi und Gebhart 1988] nach einer impulsartigen Erhöhung oder Reduktion der Wärmestromdichte temporär großskalige Wirbelstrukturen zu visualisieren, die im weiteren zeitlichen Verlauf wieder vollständig abklangen. Auch bei Temperatur- und Geschwindigkeitsmessungen mit Hilfe von reaktionsschnellen Thermoelementen und Faserfilm-Anemometern konnten irreguläre Schwankungen innerhalb der Grenzschicht festgestellt werden. Lokale Fluktuationen der Oberflächentemperatur zeigten darüber hinaus vorübergehende Abweichungen der konvektiven Wärmeübertragung von einer quasi-stationären zeitlichen Entwicklung. [Inagaki und Komori 1995] beschreiben in ähnlicher Weise das zufällige Auftreten von zweidimensionalen turbulenten Strukturen. [Zhao et al. 2015] konnten die zeitliche Variation der lokalen Auftriebsgeschwindigkeiten nach einer sprunghaften Erhöhung der Wärmstromdichte einer beheizten vertikalen Platte auch mit Hilfe einer Particle Image Velocimetry quantifizieren. Sie unterteilen die Strömungsentwicklung in einen eindimensionalen Anlaufvorgang (I), gefolgt von instationären Fluktuationen (II), die schließlich in einen quasi-stationären Endzustand (III) übergehen. Abbildung 2.1 veranschaulicht dies anhand der Geschwindigkeitskomponente $u_{\mathrm{x}}$ (Hauptströmungsrichtung).

Zusammenfassend beschreiben die dargestellten Untersuchungen irreguläre Fluktuationen der Zustandsgrößen in sämtlichen Grenzschichtbereichen (laminar, transient und turbulent) von freien Konvektionsströmungen, die selbst einer zeitlichen Veränderung unterliegen.

Eine Methodik zur Prognose der damit einhergehenden zeitlichen Entwicklung des Wärmetransports auf der Grundlage einer algebraischen oder analytischen Herangehensweise ist zum gegenwärtigen Zeitpunkt jedoch nicht bekannt. Gleichwohl würde eine derartige Methodik zahlreiche prozesstechnische Möglichkeiten eröffnen. Beispielhaft sei die Beeinflussung des Wärmeübertragungsvermögens von beheizten Oberflächen durch eine systematische Variation der Betriebsweise genannt. Das Ziel der nachfolgenden Untersuchung ist 
daher die Erarbeitung eines Berechnungsmodells zur Prognose der Wärmeübertragung durch instationäre freie Konvektion an der vertikalen Platte.

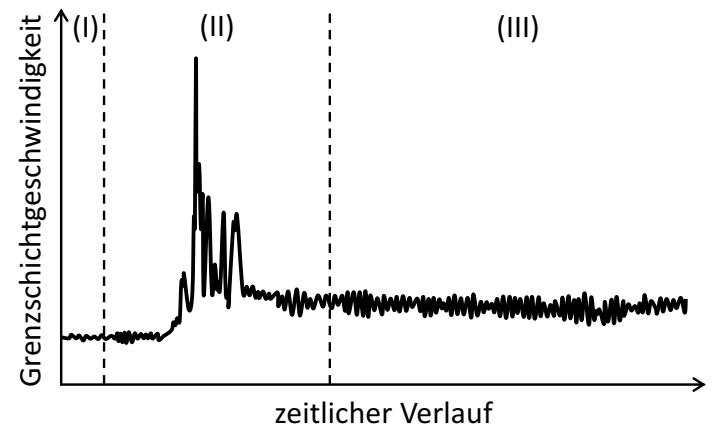

Abbildung 2.1: Lokale Grenzschichtgeschwindigkeit bei instationärer freier Konvektion

(mit I: Anlauf, II: instationäre Phase, III: quasi-stationärer Endzustand)

(Bildquelle: bearbeitet nach [Zhao et al. 2015])

\subsection{Berechnung von instationären Strömungsgrößen}

Für die Prognose von Strömungszuständen und den damit verbundenen Transportgrößen werden neben den in Kapitel 1.1 beschriebenen empirischen Korrelationen insbesondere bei anspruchsvollen Fragestellungen auch numerische Strömungssimulationen eingesetzt. Grundsätzlich kann dabei für eine beliebige Strömungskonfiguration der Transport von Masse, Impuls und Energie vollständig beschrieben werden, weshalb eine simulationsbasierte Analyse der instationären konvektiven Wärmeübertragung naheliegt. Insbesondere für die Betrachtung von freien Konvektionsströmungen treten unter den zur Durchführung derartiger Simulationen erforderlichen Vereinfachungen jedoch methodische Einschränkungen auf, deren Relevanz nachfolgend diskutiert wird.

Voraussetzung für die vollständige Beschreibung der Transportvorgänge ist eine zeitliche und räumliche Diskretisierung des gesamten Strömungsgebiets in Berechnungselemente in der Größenordnung der Kolmogorov-Skalen $(R e=1)$. Die Austauschvorgänge zwischen den Berechnungselementen resultieren dann aus einer direkten numerischen Lösung der instationären, dreidimensionalen Erhaltungsgleichungen (engl. direct numerical simulation - DNS). Mit der Kon- 
tinuitätsgleichung (Gleichung 14), den Impulserhaltungsgleichungen (Gleichungen 15 bis 17) und der Energieerhaltungsgleichung (Gleichung 18) entsteht ein gekoppeltes nichtlineares Differentialgleichungssystem (für eine ausführliche Herleitung vgl. z.B. [Herwig und Schmandt 2015]).

$$
\begin{aligned}
& \frac{\partial \rho}{\partial t}+\frac{\partial(\rho \cdot u)}{\partial x}+\frac{\partial(\rho \cdot v)}{\partial y}+\frac{\partial(\rho \cdot w)}{\partial z}=0 \\
& \frac{\partial(\rho \cdot u)}{\partial t}+\frac{\partial\left(\rho \cdot u^{2}+p-\sigma_{\mathrm{xx}}\right)}{\partial x}+\frac{\partial\left(\rho \cdot u \cdot v-\sigma_{\mathrm{yx}}\right)}{\partial y}+\frac{\partial\left(\rho \cdot u \cdot w-\sigma_{\mathrm{zx}}\right)}{\partial z}-f_{\mathrm{x}}=0 \\
& \frac{\partial(\rho \cdot v)}{\partial t}+\frac{\partial\left(\rho \cdot v \cdot u-\sigma_{\mathrm{xy}}\right)}{\partial x}+\frac{\partial\left(\rho \cdot v^{2}+p-\sigma_{\mathrm{yy}}\right)}{\partial y}+\frac{\partial\left(\rho \cdot v \cdot w-\sigma_{\mathrm{zy}}\right)}{\partial z}-f_{\mathrm{y}}=0 \\
& \frac{\partial(\rho \cdot w)}{\partial t}+\frac{\partial\left(\rho \cdot w \cdot u-\sigma_{\mathrm{xz}}\right)}{\partial x}+\frac{\partial\left(\rho \cdot w \cdot v-\sigma_{\mathrm{yz}}\right)}{\partial y}+\frac{\partial\left(\rho \cdot w^{2}+p-\sigma_{\mathrm{zz}}\right)}{\partial z}-f_{\mathrm{z}}=0 \\
& \frac{\partial(\rho \cdot H-p)}{\partial t}+\frac{\partial\left(\rho \cdot u \cdot H+\dot{\mathrm{q}}_{\mathrm{x}}\right)}{\partial x}+\frac{\partial\left(\rho \cdot v \cdot H+\dot{q}_{\mathrm{y}}\right)}{\partial y}+\frac{\partial\left(\rho \cdot w \cdot H+\dot{q}_{\mathrm{z}}\right)}{\partial z} \\
& -\left(u \cdot f_{\mathrm{x}}+v \cdot f_{\mathrm{y}}+w \cdot f_{\mathrm{z}}\right)-\mathcal{D}=0
\end{aligned}
$$

Darin gibt der erste Index der Spannungen $\sigma$ jeweils die Normalenrichtung der Oberfläche an, auf der die Spannung wirkt; der zweite Index beschreibt die Richtung der resultierenden Kraft. Die volumenbezogenen Kräfte $f$ beinhalten Schwerkraft, Zentrifugalkraft sowie die Lorentz-Kräfte. Ferner sind die Oberflächenkräfte aufgrund von Normal- und Schubspannungen in der Energiegleichung durch einen Diffusionsterm $\mathcal{D}$ gemäß Gleichung 19 zusammengefasst.

$$
\mathcal{D}=\frac{\partial\left(u \cdot \sigma_{\mathrm{xx}}+v \cdot \sigma_{\mathrm{yx}}+w \cdot \sigma_{\mathrm{zx}}\right)}{\partial x}+\frac{\partial\left(u \cdot \sigma_{\mathrm{xy}}+v \cdot \sigma_{\mathrm{yy}}+w \cdot \sigma_{\mathrm{zy}}\right)}{\partial y}+\frac{\partial\left(u \cdot \sigma_{\mathrm{xz}}+v \cdot \sigma_{\mathrm{yz}}+w \cdot \sigma_{\mathrm{zz}}\right)}{\partial z}
$$

\subsubsection{Physikalische Vereinfachungen}

Zur Lösung des Gleichungssystems sind jedoch weitere Randbedingungen zu definieren (vgl. z.B. [Oertel jr. 2017]). So gelingt die Bestimmung des Druckes durch eine Betrachtung als ideales Gas. Stoffwerte können aus kalorischen Zustandsgleichungen bestimmt und (mit Ausnahme der Dichte) vereinfachend als konstant angenommen werden. Unter der Voraussetzung eines Fourier'schen Wärmeleitungsverhaltens gelingt die Kopplung zwischen Wärmestromvektor und Temperaturfeld, wobei ein Strahlungswärmeaustausch des Fluids selbst 
nicht berücksichtigt wird. Außer der Schwerkraft werden darüber hinaus alle volumenbezogenen Kräfte vernachlässigt. Ferner kann der Spannungstensor für Newton'sche Fluide an das Geschwindigkeitsfeld gekoppelt werden, wodurch die Navier-Stoke'schen Gleichungen entstehen.

Eine elementare Reduktion der Komplexität der Transportgleichungen ergibt sich schließlich bei Verwendung der Oberbeck-Boussinesq-Approximation. Sie ermöglicht es, freie Konvektionsströmungen mit geringen Mach-Zahlen als inkompressibel zu behandeln, indem eine Veränderung der Dichte nur noch im Auftriebsterm der Impulsgleichung berücksichtigt wird. Durch den Abbruch einer Taylor-Reihe zum Zustand der Dichte $\rho$ nach dem ersten Glied gelingt eine lineare, druckunabhängige Kopplung an die Temperatur $T$ gemäß Gleichung 20.

$$
\rho(T)=\rho_{0} \cdot\left[1-\beta \cdot\left(T-T_{0}\right)\right]
$$

Zur weiteren Verringerung des Berechnungsaufwandes kann für freie Konvektionsströmungen aufgrund der niedrigen Geschwindigkeiten die viskose Dissipation in der Energiegleichung vernachlässigt werden (vgl. [Schlichting und Gersten 2006]). Bleiben für den Spezialfall der vertikalen Platte ferner die Komponenten der $z$-Achse unberücksichtigt, ergeben sich die zweidimensionalen Grundgleichungen für freie Konvektionsströmungen gemäß den Gleichungen 21 bis 24 .

$$
\begin{aligned}
& \frac{\partial u}{\partial x}+\frac{\partial v}{\partial y}=0 \\
& \rho\left(\frac{\partial u}{\partial t}+u \frac{\partial u}{\partial x}+v \frac{\partial u}{\partial y}\right)+\frac{\partial p}{\partial x}-\rho \cdot g \cdot \beta \cdot\left(T-T_{0}\right)-\mu\left(\frac{\partial^{2} u}{\partial x^{2}}+\frac{\partial^{2} u}{\partial y^{2}}\right)=0 \\
& \rho\left(\frac{\partial v}{\partial t}+u \frac{\partial v}{\partial x}+v \frac{\partial v}{\partial y}\right)+\frac{\partial p}{\partial y}-\mu\left(\frac{\partial^{2} v}{\partial x^{2}}+\frac{\partial^{2} v}{\partial y^{2}}\right)=0 \\
& \rho \cdot c_{\mathrm{p}}\left(\frac{\partial T}{\partial t}+u \frac{\partial T}{\partial x}+v \frac{\partial T}{\partial y}\right)-\lambda\left(\frac{\partial^{2} T}{\partial x^{2}}+\frac{\partial^{2} T}{\partial y^{2}}\right)=0
\end{aligned}
$$

Aus Gleichung 22 wird ersichtlich, dass bei freier Konvektion eine direkte Kopplung zwischen Temperatur- und Geschwindigkeitsfeld zu berücksichtigen ist. Energie- und Impulsgleichung können folglich nicht unabhängig voneinander gelöst werden. 
Zur Durchführung von direkten numerischen Simulationen ist jedoch auch mit den genannten Vereinfachungen und unter Berücksichtigung der rasanten informationstechnologischen Entwicklung auf absehbare Zeit die Verfügbarkeit von Großrechenanlagen (Super-Computer-Cluster) vorauszusetzen (vgl. z.B. [Oertel jr. 2017]). Selbst mit deren Rechenleistung sind die Möglichkeiten zur Betrachtung von Strömungen mit hohen Turbulenzintensitäten (große Grashofoder Reynolds-Zahlen) unter heutigen Maßstäben jedoch stark begrenzt. Darüber hinaus ergeben sich bzgl. der Verarbeitung der enormen Datenmengen weitere Problemstellungen.

Die wenigen bislang bekannten direkten numerischen Simulationen zu instationären freien Konvektionsströmungen an der vertikalen Platte konnten die in Kapitel 2.1 beschriebenen instationären Phänomene dessen ungeachtet jedoch mit sehr guter qualitativer und quantitativer Übereinstimmung abbilden (vgl. [Brooker et al. 2000], [Lin et al. 2008] sowie [Aberra et al. 2012]).

\subsubsection{Modellbasierte Vereinfachungen}

Um die genannten Restriktionen von direkten numerischen Simulationen zu umgehen und die Durchführung von Strömungssimulationen auch mit moderaten Rechenkapazitäten zu ermöglichen, wurden verschiedene (meist semiempirische) Strömungsmodelle erarbeitet, die den Berechnungsumfang zur Auswertung der strömungsmechanischen Grundgleichungen massiv reduzieren.

Im Fokus dieser Herangehensweise stehen Abstraktionen für den Transport von turbulenten Strömungsgrößen (Turbulenzmodelle). Einen wesentlichen Ansatz stellt die Modellierung von turbulenten Spannungen als äquivalente Viskosität dar (Wirbelviskositäts-Modelle oder Boussinesq-Ansatz). Voraussetzung für derartige Modelle ist eine (i.d.R. zeitliche) Mittelung von Strömungsparametern, aus der die Reynolds-gemittelten Navier-Stokes-Gleichungen (engl. Reynolds-averaged Navier-Stokes - RANS) hervorgehen. Darauf aufbauend existieren sowohl Modelle, die Isotropie voraussetzen, als auch solche, die eine Prognose von anisotropen Strömungen ermöglichen. Erstere approximieren die turbulenten Schubspannungen proportional zum mittleren Geschwindigkeitsgradienten, z.B. in Form von Zweigleichungsmodellen. Anisotrope Transportvor- 
gänge werden durch eine Modellierung der Umverteilung der Turbulenz selbst, u.a. in Reynolds-Stress-Transport-Modellen (RSTM), abgebildet.

Darüber hinaus kann der RANS-Ansatz mit Hilfe einer Ensemblemittelung grundsätzlich auch in statistisch nicht stationären Strömungen angewendet werden (engl. unsteady RANS - URANS). Für eine turbulente Strömungsgröße, die im statistisch stationären Fall typischer Weise eine zufällige Schwankung um einen Mittelwert aufweist, wird dann anstelle einer zeitlichen Mittelung für jeden Zeitschritt das arithmetische Mittel aus einer mehrmaligen Berechnung gebildet. Dies ermöglicht eine Prognose der zeitlichen Entwicklung von mittleren Strömungsgrößen. URANS-Methoden können jedoch folglich nur eine hinreichende Grundlage zur Beurteilung von instationären Phänomenen sein, solange eine Trennung zwischen turbulenzbedingten Schwankungen und den instationären Änderungen selbst möglich ist (sog. spektrale Lücke, vgl. z.B. [Wilcox 2002]). Anschaulich ausgedrückt, werden durch URANS-Methoden also nur großräumige Wirbel, nicht jedoch die kleineren Strukturen (Turbulenzballen) erfasst, da diese durch den Mittelungsprozess verschwinden.

Ein alternatives Vorgehen besteht in der direkten numerischen Simulation von großskaligen Wirbeln in Kombination mit Modellen für kleinskalige Strukturen (engl. large-eddy simulation - LES). Dem liegt die Vorstellung zugrunde, dass die größten Turbulenzelemente am stärksten zur Produktion sowie zur anisotropen Verteilung der turbulenten kinetischen Energie beitragen und maßgeblich für die Strömungsgestalt sind. Kleinskaligen turbulenten Strukturen werden hingegen hauptsächlich Dissipationsvorgänge zugeordnet; sie weisen daher einen eher universellen Charakter auf. Da die räumlich gefilterten großskaligen Turbulenzelemente ohne Modellannahmen simuliert werden, entfallen die Notwendigkeit einer Mittelung und somit auch die o.g. Restriktionen der URANS-Methodik.

Als problematisch ist bei derartigen Grobstruktursimulationen jedoch die Berücksichtigung der Wechselwirkungen zwischen den kleinen, nicht numerisch aufgelösten und den großskaligen, aufgelösten Strömungselementen anzusehen. So liegt den Feinstruktur-Modellen (engl. subgrid-scale - SGS) u.a. die Hypothese einer lokalen Isotropie zugrunde. Im Gegensatz zu ungestörten Strömungsbereichen ergibt sich bei Grenzschicht-Strömungen jedoch insbesondere im für den Wärmeübergang relevanten wandnahen Bereich aufgrund der fes- 
ten Begrenzung zwangsläufig eine Richtungsabhängigkeit. Die turbulenten Bewegungen werden dabei in Richtung der Wand aufgrund von Schubspannungen zunehmend gedämpft, bis schließlich die molekulare Viskosität dominiert. Darüber hinaus findet im Fall von freien Konvektionsströmungen aufgrund des Energietransports von der Wand an das Fluid eine Umkehr des Energieflusses statt. So wird im wandnahen Bereich aufgrund von thermischer Instabilität turbulente kinetische Energie in den kleinen, nicht numerisch aufgelösten Skalen produziert und zu den großen, aufgelösten Skalen transportiert (vgl. z.B. [Fröhlich 2006]).

Berücksichtigt werden derartige Strömungsformen durch hybride Ansätze. Die LES wird dabei im wandnahen Bereich durch zusätzliche Methoden ergänzt. Beispielhaft seien hier die Wandfunktionen sowie Niedrig-Reynolds-ZahlTurbulenzmodelle (engl. low-Re) genannt. Für letztere (im Zusammenhang mit LES oft als DES - engl. detached-eddy simulation - bezeichnet) sind ebenfalls die Einschränkungen der Reynolds-Mittelung in instationären Strömungen zu beachten. Die meist algebraischen Zusammenhänge zwischen Wandabstand und lokaler Geschwindigkeit oder Schubspannung von Wandfunktionen setzen indessen eine konstante turbulente Wirbelviskosität, also eine vollständig ausgebildete, statistisch stationäre Strömung voraus (vgl. [Schlichting und Gersten 2006]). Darüber hinaus weisen beide Ansätze bei einer Anwendung in freier und Mischkonvektion aufgrund der Kopplung von Temperatur- und Strömungsfeld bereits im stationären Fall größere Unsicherheiten auf (vgl. [Henkes und Hoogendoorn 1990] sowie [Xu et al. 1998]) und erfordern zusätzliche Modellanpassungen (vgl. z.B. [Kriegel 2005] und [Hölling 2006]).

Alternativ ist bei LES-Methoden eine lokale Anpassung der numerischen Auflösung und der räumlichen Filterung möglich, sodass auch im relevanten wandnahen Bereich sehr kleinskalige Strömungselemente ohne weitere Modellannahmen simuliert werden. Eine derartige Verfeinerung der numerischen Diskretisierung führt jedoch ebenfalls zu einem enormen Berechnungsaufwand, der letztlich in einer quasi-direkten numerischen Simulation mündet. 


\subsection{Weiterführende Betrachtung der instationären Wärme- übertragung}

Aufgrund der in Kapitel 2.2 genannten Restriktionen einer simulationsbasierten Untersuchung der Wärmeübertragung durch instationäre freie Konvektionsströmungen werden mögliche Kausalzusammenhänge zwischen einer gezielten Variation von Strömungs-Randbedingungen und der instationären Wärmeübertragungs-Intensität im Folgenden experimentell analysiert. Eine Beschreibung des Versuchsaufbaus und der Bewertungsmethodik sowie eine Darstellung und Interpretation der Messergebnisse erfolgen in Kapitel 3. Ergänzend werden die auftretenden Strömungscharakteristika in Kapitel 4 visualisiert und phänomenologisch interpretiert. Auf Grundlage der gewonnenen Erkenntnisse wird in Kapitel 5 schließlich ein analytisches Modell zur Prognose der Wärmeübertragung durch instationäre freie Konvektionsströmungen an der vertikalen Platte vorgestellt und im Rahmen von gezielten Wiederholungsmessungen validiert. 


\section{Experimentelle Analyse der Wärmeübertragung durch freie Konvektion an der vertikalen Platte}

Zur experimentellen Analyse der Auswirkungen einer instationären freien Konvektionsströmung auf die Wärmeübertragung von einer vertikalen Platte an Luft wird eine direkt-elektrisch beheizte Oberfläche nachfolgend stationären sowie zeitlich variablen (instationären) Betriebsmodi ausgesetzt. Als Bewertungsgrundlage dient dabei der Vergleich zwischen einer quasi-stationären Betrachtung und den bilanzierten Größen im instationären Fall (siehe Kapitel 3.3). Die verwendeten Stoffwerte beziehen sich jeweils auf die arithmetisch gemittelte Temperatur der Plattenoberfläche und des ungestörten Umgebungsfluids. Eine Ausnahme stellt der Volumen-Ausdehnungskoeffizient $\beta$ dar. Im Sinne einer Kopplung von Fluiddichte und Fluidtemperatur (vgl. Gleichung 20) ist für dessen Bestimmung nur das Umgebungs-Temperaturniveau von Relevanz.

\subsection{Versuchsaufbau}

Insbesondere für die messtechnische Erfassung von instationären Größen leiten sich verschiedene Anforderungen an die Beschaffenheit eines experimentellen Aufbaus ab. Vorrangig ist hier die Berücksichtigung des Einflusses von thermischen Kapazitäten der wärmeübertragenden Oberfläche sowie der verwendeten Messtechnik zu nennen. Darüber hinaus liegen der konstruktiven Ausgestaltung des Versuchsaufbaus die nachfolgend aufgeführten funktionellen Erfordernisse zugrunde.

- Berücksichtigung sämtlicher Strömungsformen, d.h. laminar, transient und vollturbulent.

- Erfassung von Momentanwerten der Wärmeübertragung im instationären Betriebsfall.

- Lokal variabel anpassbare Leistungsdichte oder Oberflächentemperatur der beheizten Platte.

- Strömungsmechanisch ungestörtes Umgebungsfluid mit nahezu konstantem Stoffzustand. 
- Möglichst geringer Einfluss anderer Wärmetransportmechanismen (insbesondere Strahlung).

\subsubsection{Konstruktion und Umgebungsbedingungen}

Als Umgebungsfluid zur Untersuchung der instationären freien Konvektion dient feuchte Luft. Der Versuchsaufbau ist dazu in einer ca. $180 \mathrm{~m}^{2}$ großen und etwa $7 \mathrm{~m}$ hohen, vollklimatisierten Halle des Hermann-Rietschel-Instituts der TU Berlin angeordnet. Die thermo-physikalischen Randbedingungen sind für die Dauer der Versuche konstant auf $20^{\circ} \mathrm{C} \pm 1 \mathrm{~K}$ und $50 \%$ r.F. $\pm 5 \%$ r.F. gehalten.

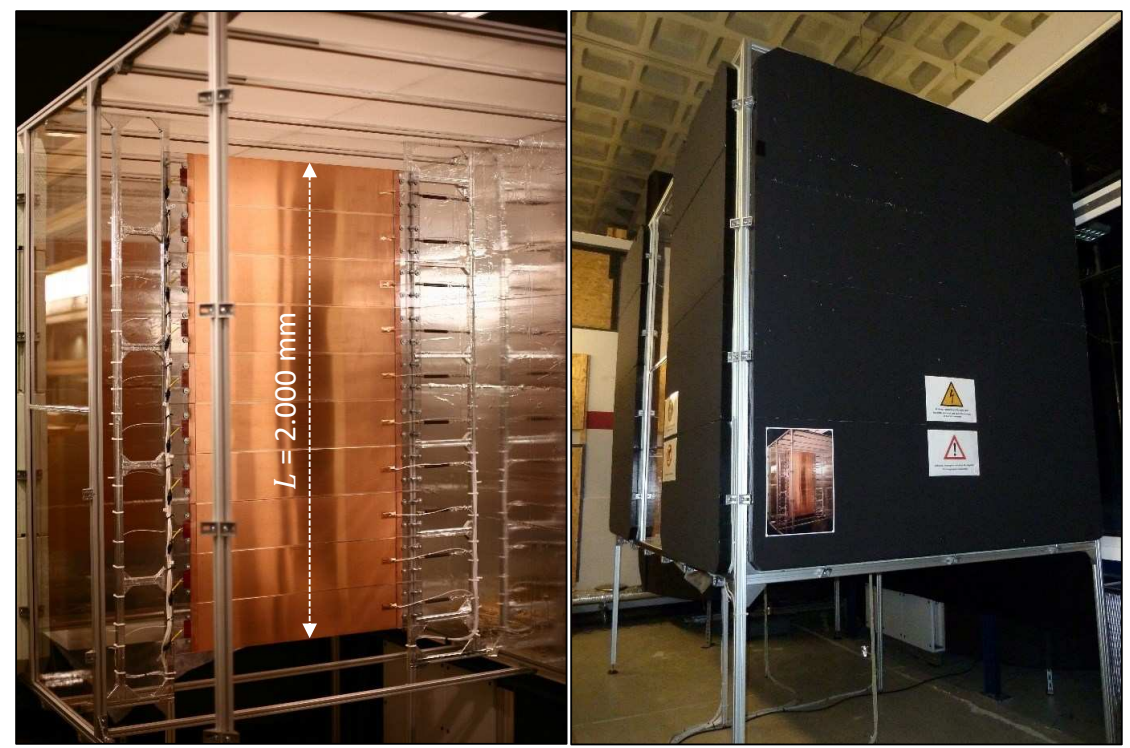

Abbildung 3.1: Strömungsmechanische Abschirmung der beheizten Platte (links: geöffnete Abschirmung; rechts: geschlossener Zustand)

Um der Anforderung eines vollturbulenten Strömungsbereichs gerecht zu werden, sind Grashof-Zahlen in der Größenordnung von $G r_{x / L}>2 \cdot 10^{10}$ erforderlich (vgl. Kapitel 1.1). Dies kann in Luft beispielweise für Temperaturdifferenzen von ca. $\Delta \vartheta_{\mathrm{W}-\infty}=70 \mathrm{~K}$ zwischen Oberfläche und Umgebungsfluid und bei einer Strömungsweglänge $a b$ ca. $x / L \geq 1,5 \mathrm{~m}$ erreicht werden. Um einen ausreichend großen vollturbulenten Bereich sicherzustellten, ist der Versuchsaufbau auf eine Strömungsweglänge von $L=2 \mathrm{~m}$ und eine maximale Übertemperatur der 
beheizten Platte von $\Delta \vartheta_{\mathrm{W}-\infty}=80 \mathrm{~K}$ ausgelegt, was einer rechnerischen GrashofZahl von ca. $G r_{\mathrm{x} / \mathrm{L}}=5,8 \cdot 10^{10}$ an der Plattenoberkante entspricht.

Zur Vermeidung einer Beeinflussung der zu betrachtenden natürlichen Konvektionsvorgänge durch die Raumluftströmungen der klimatechnischen Konditionierung der Versuchshalle, ist die beheizte Platte horizontal allseitig strömungsmechanisch abgeschirmt (Abbildung 3.1). Die Abschirmung besteht aus einer Dämmschicht mit geringer thermischer Kapazität (120 mm extrudierter Polystyrol-Hartschaum). Sie ist zudem in Richtung der beheizten Platte mit einer stark reflektierenden Aluminium-Folie beschichtet, um den Strahlungswärmeaustausch mit der Platte zu minimieren. In Richtung der Versuchshalle ist die Abschirmung matt schwarz lackiert, um möglichst im thermischen Gleichgewicht mit den Umgebungsbedingungen zu stehen.

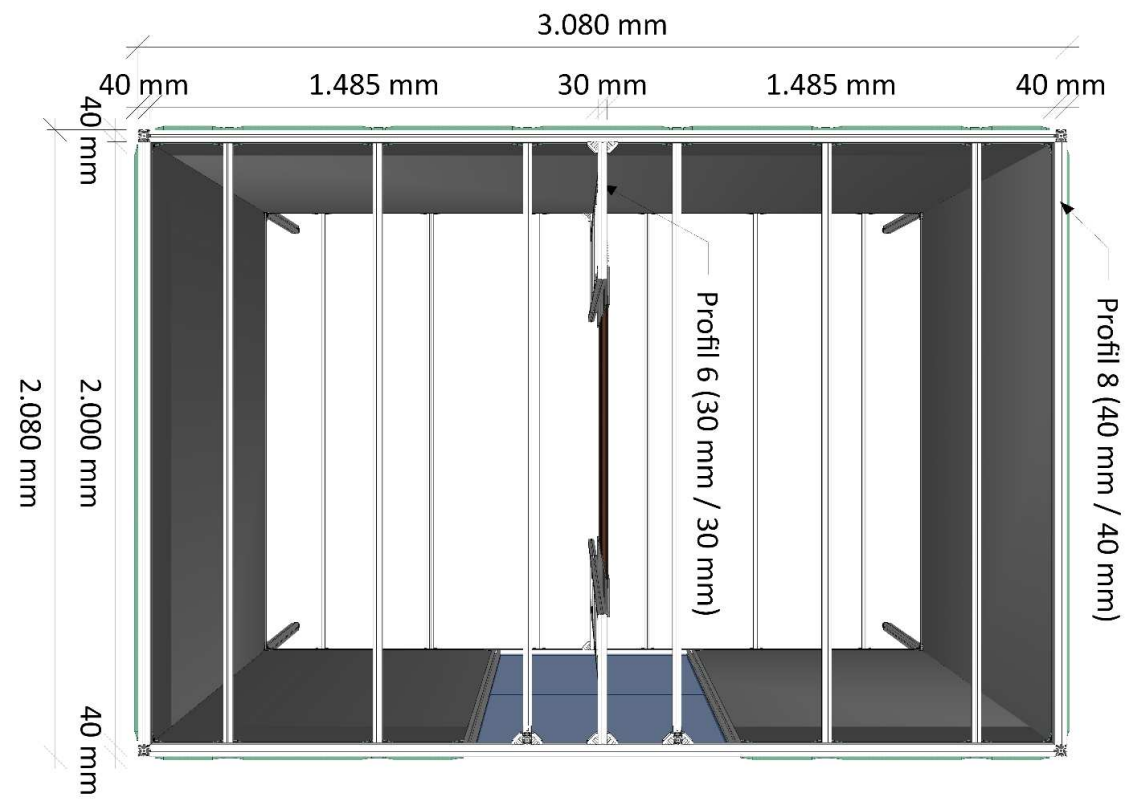

Abbildung 3.2: Abmessungen des Versuchsaufbaus (Draufsicht)

In vertikaler Richtung ist die Abschirmung durch $50 \mathrm{~mm}$ hohe StrömungsGleichrichter ausgeführt. Diese bestehen aus extrudiertem Polypropylen und weisen eine wabenartige Struktur auf, die durch nebeneinander angeordnete, vertikale Röhren mit einem Innendurchmesser von jeweils $8 \mathrm{~mm}$ entsteht und 
einen freien Querschnitt von ca. 85 \% aufweist. Die Auftriebsströmung der beheizten Platte kann so nach oben aus dem abgeschirmten Bereich austreten, während von unten Umgebungsluft aus der Versuchshalle nachströmt.

Die beheizte Platte besteht aus zehn übereinander angeordneten KupferSandwich-Elementen (siehe Kapitel 3.1.2) mit einer Höhe von jeweils 200 mm und einer Breite von einem Meter.

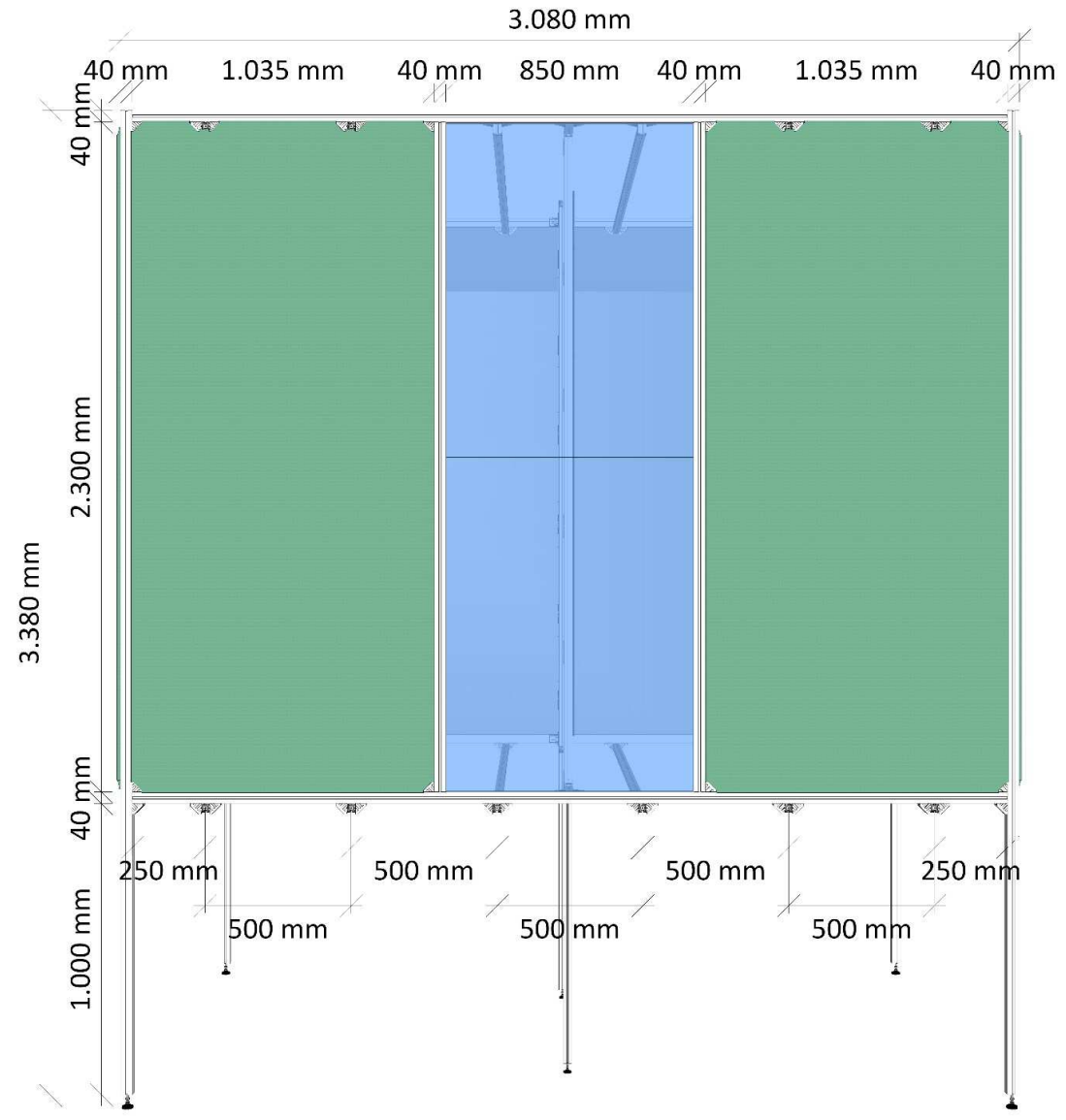

Abbildung 3.3: Abmessungen des Versuchsaufbaus (Seitenansicht)

Orthogonal zur Plattenebene befindet sich an einer Seite der horizontalen Abschirmung eine $850 \mathrm{~mm}$ breite Borosilikat-Glasscheibe mit einer Dicke von 
$5 \mathrm{~mm}$. Sie ermöglicht die optische Erfassung von Strömungsvorgängen (Kapitel 4.1) bei geringem Brechungsindex (ca. 1,47) und weist ebenfalls einen hohen Reflexionsgrad im infraroten Bereich auf.

Die Gesamtabmessungen des Versuchsaufbaus sind in Abbildung 3.2 und Abbildung 3.3 dargestellt.

\subsubsection{Sandwich-Heizelemente}

Zur Bestimmung von Momentanwerten der Intensität der konvektiven Wärmeübertragung ist eine Berücksichtigung der thermischen Kapazität der beheizten Platte erforderlich, da ein Teil des bilanzierten Wärmestroms deren Be- oder Entladung zuzurechnen ist (siehe Kapitel 3.3.2). Im Falle einer einseitig an ein Umgebungsfluid grenzenden Oberfläche, die rückseitig durch starke Dämmung als annähernd adiabat betrachtet werden kann, gelänge dies nur mit Kenntnis des zeitvariablen Temperaturprofils innerhalb der Dämmschicht.

Den durchgeführten experimentellen Untersuchungen liegt daher ein symmetrischer Aufbau zu Grunde, der beidseitig an das Umgebungsfluid grenzt. Abbildung 3.4 zeigt die Konstruktion der Sandwich-Heizelemente schematisch.

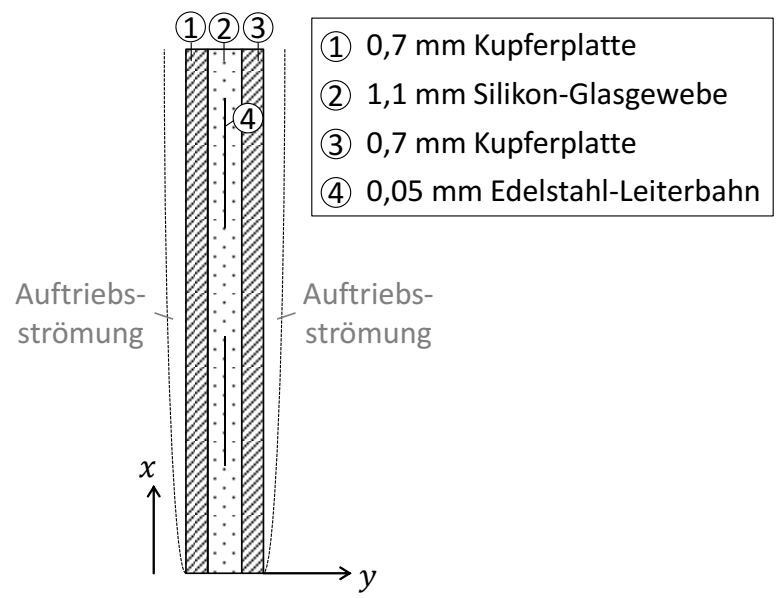

Abbildung 3.4: Aufbau der Sandwich-Heizelemente

Dabei dient ein ca. 1,1 mm dickes Silikon-Glasgewebe als Trägermaterial für Edelstahl-Leiterbahnen mit einer Breite von etwa 4,2 mm und einer Dicke von 
0,05 mm. Die Leiterbahnen sind im Abstand von rund 3,5 mm im ätztechnischen Prinzip angeordnet, woraus eine Belegungsdichte von gut $55 \%$ resultiert. Auf dem Trägermaterial der Heizleiter ist mittels Verklebung beidseitig eine 0,7 mm dicke Kupferplatte (Typ Cu-DHP_CW024A) angebracht, die eine homogene Temperaturverteilung in der Plattenebene bewirkt und ihrerseits einen sehr geringen Emissionskoeffizienten für Wärmestrahlung aufweist. Die Homogenität der Oberflächentemperatur wird für den genannten Aufbau zuvor messtechnisch mit Hilfe einer Infrarot-Thermografie an einem matt-schwarz beschichteten Probe-Heizelement überprüft. Dabei sind sowohl im stationären Fall, als auch während eines Aufheizvorganges keine nennenswerten lokalen Temperaturunterschiede feststellbar. Aufgrund der geringen Gesamtdicke der Sandwich-Heizelemente von etwa 2,5 mm und der hohen Wärmeleitfähigkeit der Kupferschichten wird auch das Temperaturprofil im Plattenquerschnitt ( $y-$ Komponente) als homogen angenommen.

Jedes der zehn Heizelemente weist einen elektrischen Wirkwiderstand von ca. $6 \Omega$ auf und kann mit einer Stromstärke von bis zu $10 \mathrm{~A}$ beaufschlagt werden. Für die dazu erforderliche Gleichspannung von $60 \mathrm{~V}$ resultiert eine Leistung von rund $600 \mathrm{~W}$ pro Sandwich-Heizelement. Dies entspricht einer flächenspezifischen elektrischen Leistung von etwa $1.500 \mathrm{~W} / \mathrm{m}^{2}$ (beidseitig).

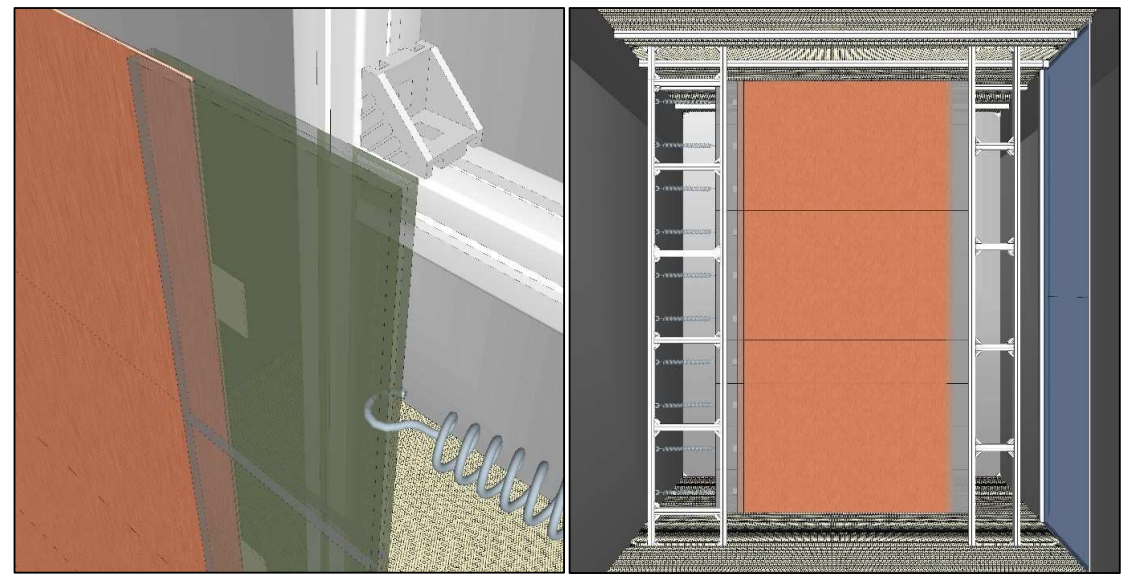

Abbildung 3.5: Befestigung der Sandwich-Heizelemente (links: Klemmvorrichtung; rechts: Gesamtaufhängung) 
Die Sandwich-Heizelemente sind einzeln mit Hilfe einer Klemmvorrichtung aus Polycarbonat (Makrolon ${ }^{\circledR}$ ) befestigt, das für Temperaturen bis $110^{\circ} \mathrm{C}$ dauerhaft beständig ist und kurzzeitig mit bis zu $135^{\circ} \mathrm{C}$ belastet werden kann. Die Lagerung der Klemmvorrichtungen ist an einer Seite der Platte beweglich ausgeführt, um die Längenausdehnung der Kupferplatten aufgrund von Temperaturänderungen auszugleichen (Abbildung 3.5). Die Zugkraft einer Feder unterstützt dabei auch während eines Aufheiz- oder Abkühlvorgangs eine möglichst ebene Plattenanordnung.

In vertikaler Richtung sind die einzelnen Sandwich-Heizelemente zur Kompensation der Materialausdehnung jeweils mit einem Abstand von ca. $1 \mathrm{~mm}$ angeordnet. Die verbleibenden Spalte sind mit einem etwa 0,06 mm dicken KupferKlebeband verschlossen, wie in Abbildung 3.6 dargestellt.

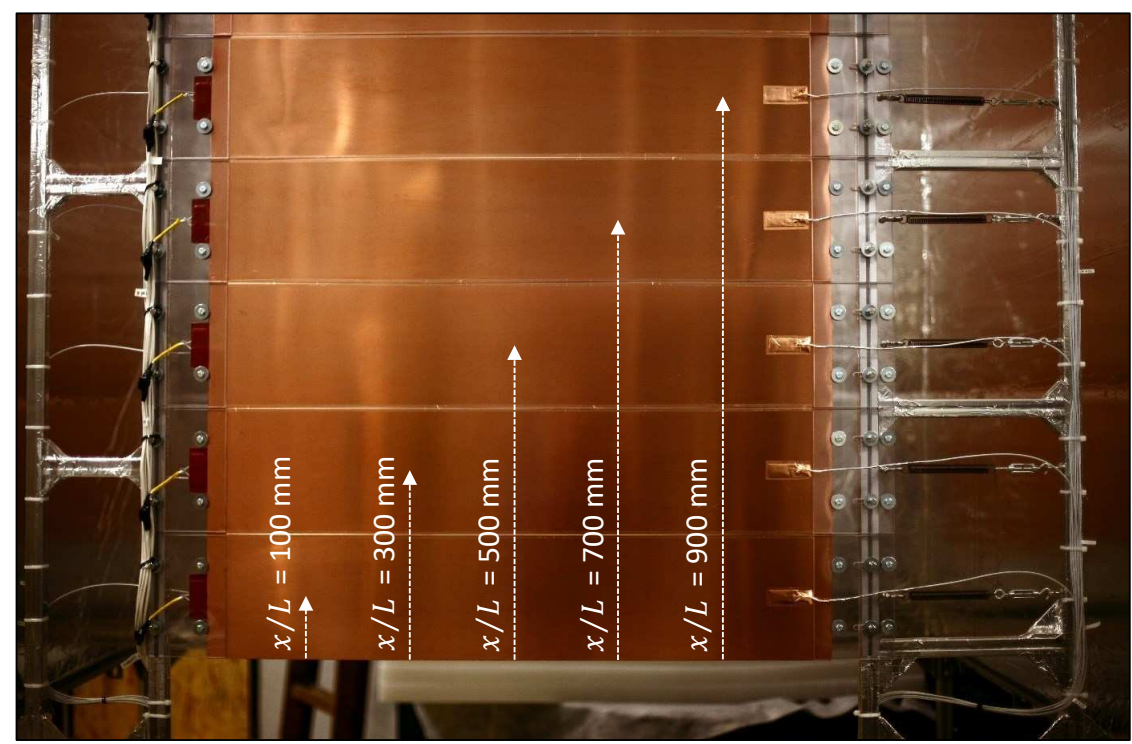

Abbildung 3.6: Ansicht der Sandwich-Heizelemente

\subsubsection{Leistungszufuhr und Regelung}

Die beschriebene Aufteilung der betrachteten vertikalen Platte in zehn Einzelelemente wird vorrangig durch die Anforderung einer lokal individuellen Anpassung der Wärmestromdichte oder der Oberflächentemperatur begründet. 
Die Versorgung der Heizelemente erfolgt durch zehn elektronische Labornetzgeräte (Typ Manson HCS 3404), wie in Abbildung 3.7 dargestellt.

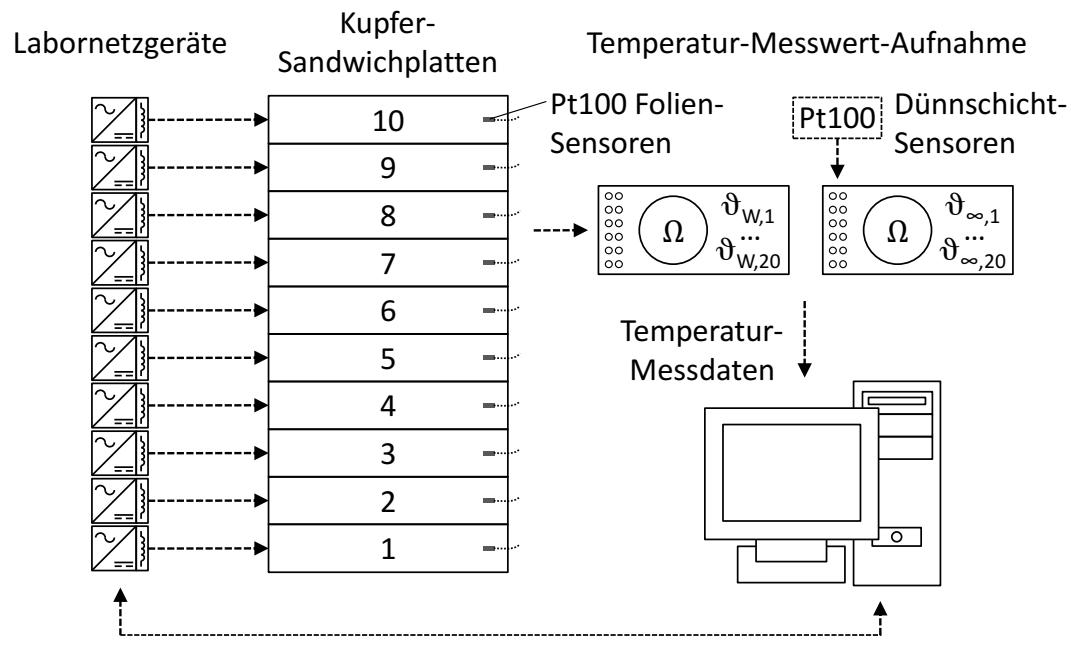

Regelung und Messung der elektrischen Leistungszufuhr

\section{Abbildung 3.7: Struktur des Mess- und Regelungssystems}

Die Netzgeräte werden mit Hilfe einer zentralen Mess-, Steuer- und Regelungsapplikation in der Entwicklungsumgebung LabVIEW ${ }^{\mathrm{TM}}$ betrieben. Für jedes der Sandwich-Heizelemente können somit nahezu beliebige zeitliche Profile der zugeführten Wärmestromdichte vorgegeben werden. Alternativ kann jedes der Sandwich-Heizelemente mit Hilfe eines eigenen Regelkreises auf eine individuelle Oberflächentemperatur geregelt werden.

Neben der Anpassung der zugeführten elektrischen Leistung dienen die Netzgeräte auch zur Messung ebendieser. Dabei wird für jede der zehn Kombinationen aus Netzgerät, elektrischer Versorgungsleitung und Heizelement eine Korrektur zwischen der eingestellten Ausgangsleistung des Netzgeräts und der tatsächlichen Leistung am Heizelement auf der Basis von individuellen Kalibrierungs-Messungen vorgenommen. Im Mittel beträgt der Spanungsabfall zwischen Netzgerät und Heizelement ca. 2,7 \% bei einer Stromstärke von $1 \mathrm{~A}$ und rund $4 \%$ bei $10 \mathrm{~A}$. Die Korrektur der zugeführten Leistung erfolgt dabei mit sehr guter Regressions-Güte durch Polynome zweiten Grades. Da die Netzgerä- 
te nach dem Konstant-Strom-Prinzip arbeiten, bleibt der relative Spannungsabfall unabhängig von einer temperaturabhängigen Veränderung des Wirkwiderstandes der Heizelemente und der Versorgungsleitungen.

\subsubsection{Messwert-Erfassung}

Zur Bestimmung der konvektiven Wärmeübertragungs-Intensität (siehe Kapitel 3.2 und 3.3) werden die Oberflächentemperaturen der Sandwich-Heizelemente messtechnisch erfasst. Dies geschieht mit Platin-Widerstandssensoren der Kategorie Pt100. Sie sind in einer $0,3 \mathrm{~mm}$ dicken, folienartigen PolyimidTrägerschicht mit einer Breite von $22 \mathrm{~mm}$ und einer Länge von $55 \mathrm{~mm}$ eingefasst (Typ KIMO SFSC50-4-TB-10). Die Sensor-Folien sind mit Hilfe von Wärmeleitpaste $\left(\lambda=8,5 \frac{\mathrm{W}}{\mathrm{m} \cdot \mathrm{K}}\right)$ auf der Oberfläche der Kupferplatten befestigt und durch ein ebenfalls 0,06 mm dickes Kupfer-Klebeband überdeckt, um eine erhöhte Strahlungswärme-Abgabe der Sensorflächen zu vermeiden (siehe auch Abbildung 3.6).

Pro Sandwich-Heizelement befindet sich ein Oberflächentemperatursensor an jeder der beiden an das Umgebungsfluid grenzenden Seiten. Dies ermöglicht eine zweifache messtechnische Erfassung der konvektiven Wärmeübertragungs-Intensität bei jedem Einzelversuch.

Die Bestimmung der Temperatur des Umgebungsfluids erfolgt mit Hilfe von Pt100-Dünnschichtsensoren (Typ Heraeus M222). Bei maximalen Abmessungen von etwa 1 bis $2 \mathrm{~mm}$ weisen diese in Luft eine Zeitkonstante von ca. $\tau=4 \mathrm{~s}$ auf. Wie in Abbildung 3.8 dargestellt ist, sind auf jeder Seite der beheizten Platte fünf Lufttemperatursensoren in einem horizontalen Abstand von ca. $750 \mathrm{~mm}$ gleichmäßig über das Höhenprofil verteilt angeordnet. Außerdem erfasst jeweils ein Sensor unmittelbar die An- und Abströmtemperaturen der Platte und die Lufttemperatur außerhalb der strömungsmechanischen Abschirmung. Zur bilanziellen Berücksichtigung der verbleibenden Strahlungswärmeabgabe der Sandwich-Heizelemente wird auch die Temperatur der gegenüberliegenden Innenoberflächen der strömungsmechanischen Abschirmung mit insgesamt vier Sensoren des gleichen Typs erfasst (vgl. Abbildung 3.8).

Um eine Beeinflussung der Lufttemperatursensoren durch WärmestrahlungsEmissionen der beheizten Platte zu vermeiden, werden diese horizontal umlau- 
fend mit einem Strahlungsschutz versehen, wie in Abbildung 3.9 dargestellt. Die Messwert-Erfassung erfolgt bei allen Temperatursensoren im VierleiterPrinzip mit geschirmten Messleitungen.

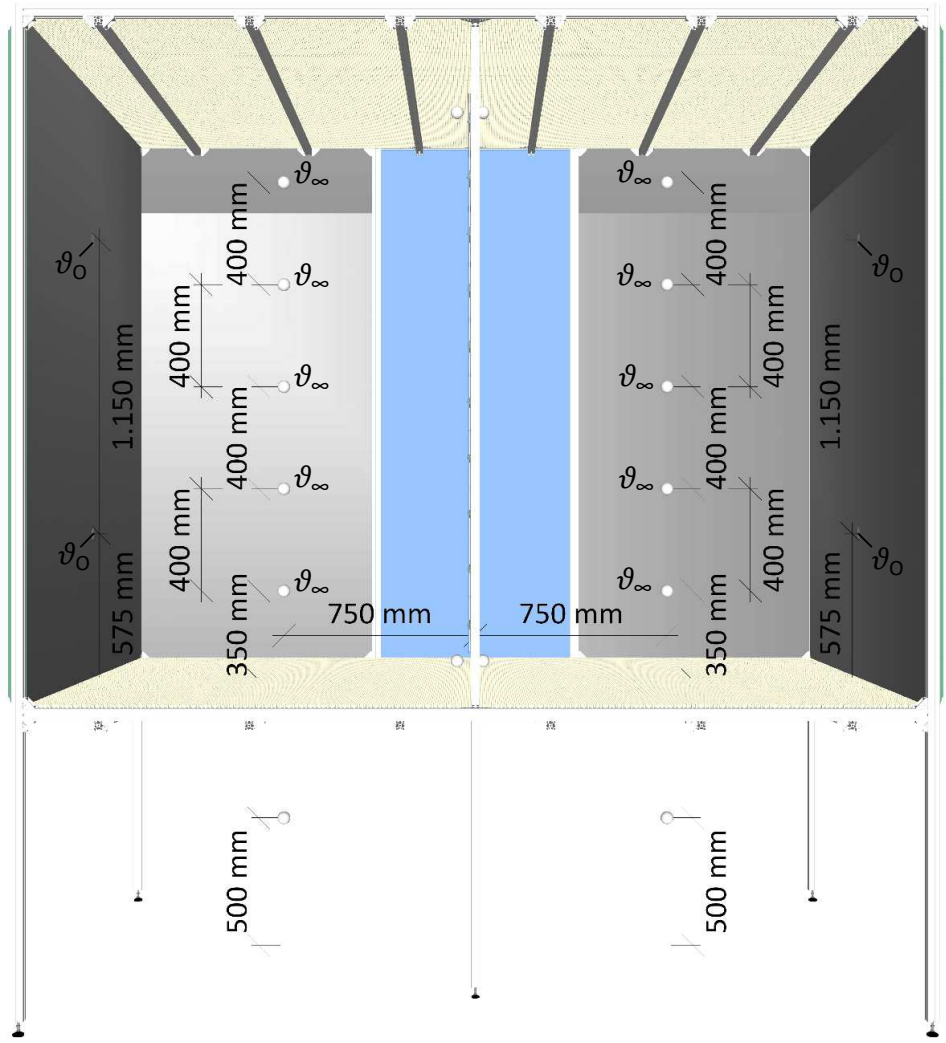

Abbildung 3.8: Anordnung der Temperatursensoren

Tabelle 3.1: Messunsicherheiten

\begin{tabular}{l|l} 
Komponente & Messunsicherheit \\
\hline Oberflächentemperatursensoren & $\pm\left(0,15^{\circ} \mathrm{C}+0,2 \%\right.$ v. MW $)$ \\
Umgebungstemperatursensoren & $\pm\left(0,1{ }^{\circ} \mathrm{C}+0,17 \%\right.$ v. MW $)$ \\
Netzgeräte & $\pm 0,2 \%$ v. MW \pm 3 LSB \\
Multimeter-Datenlogger & $\pm(0,01 \%$ v. MW $+0,004 \%$ v. MB $)$
\end{tabular}




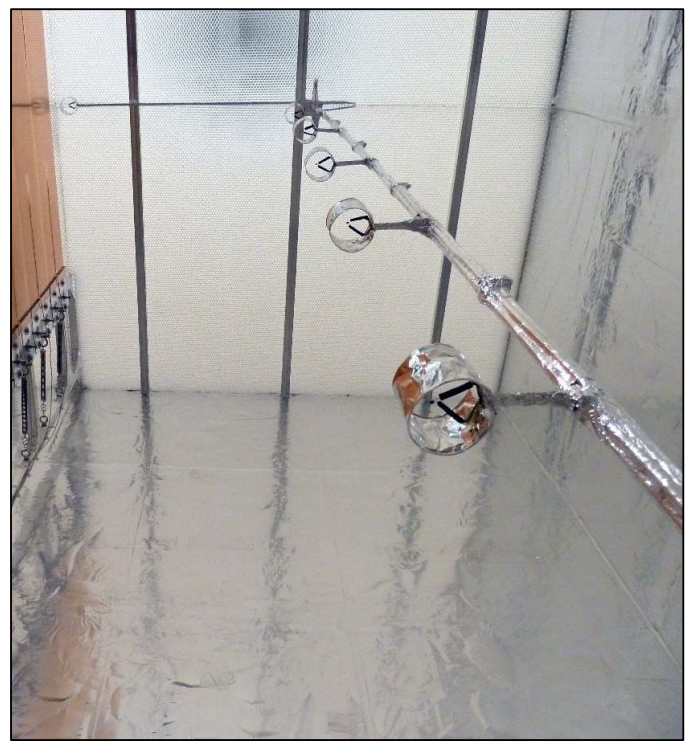

Abbildung 3.9: Strahlungsabschirmung der Lufttemperatursensoren

Als Messwert-Aufnehmer dienen zwei Multimeter-Datenlogger vom Typ Agilent 34970A mit je zwei Multiplexer-Modulen (Typ Keysight 34901A). Das Mess- und Regelungs-Intervall liegt für sämtliche Versuchsreihen bei $2 \mathrm{~s}$. Tabelle 3.1 gibt eine Übersicht der relevanten Messunsicherheiten; eine Betrachtung der Messunsicherheits-Fortpflanzung erfolgt in Kapitel 3.3.4.

\subsubsection{Betriebsverhalten}

In Abbildung 3.10 ist der Aufheizvorgang für die maximale konvektive Wärmestromdichte der Sandwich-Heizelemente von ca. $\dot{q}_{\mathrm{c}, \mathrm{st}}=1.430 \mathrm{~W} / \mathrm{m}^{2}$ (siehe Gleichung 27 in Kapitel 3.2.2) bis zu einer Oberflächentemperatur von knapp $\vartheta_{\mathrm{W}}=100^{\circ} \mathrm{C}$ sowie das anschließende Auskühlverhalten ohne Leistungszufuhr dargestellt. Die Aufheizdauer beträgt dabei etwa $270 \mathrm{~s}$. Bezogen auf den Zeitraum mit der stärksten Änderung $\left(63,2 \%\right.$ von $\Delta \vartheta_{\mathrm{W}}$ innerhalb $\left.1 \tau\right)$ liegt die mittlere Aufheizgeschwindigkeit bei rund ${\overline{\partial \vartheta_{\mathrm{W}} / \partial t_{1 \tau}}}_{1 \mathrm{~T}}=0,33 \mathrm{~K} / \mathrm{s}$ und die mittlere Auskühlgeschwindigkeit bei ca. ${\overline{\partial \vartheta_{\mathrm{W}} / \partial t_{1 \tau}}}_{1}=0,055 \mathrm{~K} / \mathrm{s}$. Die Totzeit der beheizten Platte liegt bei deutlich unter $1 \mathrm{~s}$. 


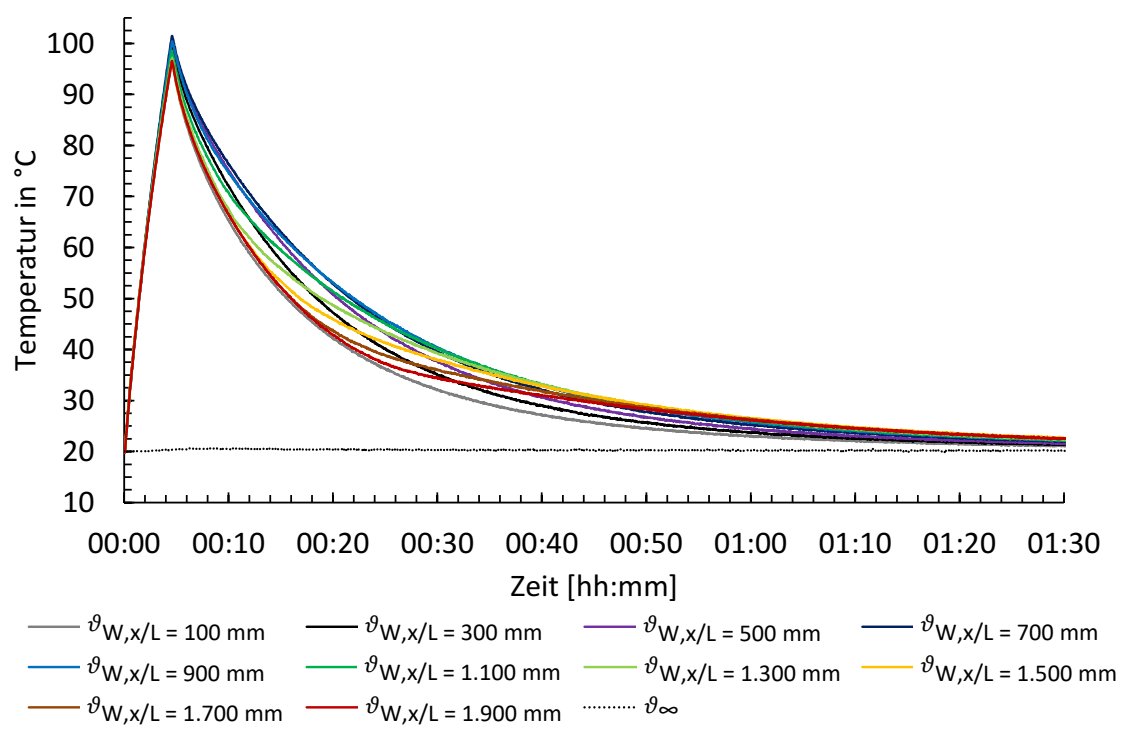

Abbildung 3.10: Aufheiz- und Auskühlverhalten der Sandwich-Heizelemente

Für die isotherme Oberfläche resultieren im stationären Fall Grashof-Zahlen von bis zu $G r_{\mathrm{L}} \approx 5,5 \cdot 10^{10}$, wodurch sämtliche Strömungsformen abgebildet werden können (vgl. Kapitel 1.1). Während des Wärmestrom-Impulses liegen kurzzeitig Grashof-Zahlen von bis zu $G r_{\mathrm{L}}^{*} \approx 2 \cdot 10^{13}$ vor.

Zu Beginn des Aufheizvorgangs weisen zunächst alle zehn SandwichHeizelemente nahezu dieselbe Oberflächentemperatur auf. Für $\vartheta_{\mathrm{W}} \geq 80^{\circ} \mathrm{C}$ sowie während des Abkühlvorgangs zeigen sich hingegen deutliche Temperaturunterschiede zwischen den einzelnen Heizelementen. Das Maximum tritt rund 12 Minuten nach Beginn des Aufheizvorgangs auf und beträgt ca. $\vartheta_{\mathrm{W}, \mathrm{x} / \mathrm{L}=700 \mathrm{~mm}}-\vartheta_{\mathrm{W}, \mathrm{x} / \mathrm{L}=100 \mathrm{~mm}} \approx 12 \mathrm{~K}$. Dies ist auf die erwartungsgemäße lokale Variation in der Intensität der konvektiven Wärmeübertragung zurückzuführen, die in den Kapiteln 3.2.3 und 3.2.4 diskutiert wird (siehe insbesondere Abbildung 3.13 sowie Abbildung 3.16).

\subsection{Stationäre Validierung}

Zur Überprüfung der Belastbarkeit von Messergebnissen des in Kapitel 3.1 beschriebenen Versuchsaufbaus wird zunächst das Wärmeübertragungsvermö- 
gen im stationären Fall erfasst. Ein Vergleich mit den Daten aus bekannten Literaturquellen dient als Validierungsgrundlage.

\subsubsection{Bestimmung der Wärmeübertragung aufgrund von Strahlung}

Der Wärmeaustausch zwischen der beheizten Platte und der strömungsmechanischen Abschirmung aufgrund von Strahlung $\dot{q}_{r}$ wird gemäß Gleichung 25 nach dem Prinzip der grauen Strahler bestimmt.

$$
\dot{q}_{\mathrm{r}}=\varepsilon \cdot 5,67 \cdot 10^{-8} \cdot\left(T_{\mathrm{W}}{ }^{4}-T_{\mathrm{O}}{ }^{4}\right)
$$

Dabei werden vereinfachend wellenlängenunabhängige Emissionskoeffizienten verwendet. So wird für die polierte Kupferoberfläche der SandwichHeizelemente (vgl. Kapitel 3.1.2) ein Emissionskoeffizient von $\varepsilon_{W}=0,03$ und für die umgebende strömungsmechanische Abschirmung mit hochglanzpolierter Aluminium-Oberfläche ein Wert von $\varepsilon_{0}=0,04$ angenommen (vgl. [VDI 2013]). Der Gesamtemissionsgrad wird auf der Grundlage der Sichtverhältnisse von sich umhüllenden Körpern gemäß Gleichung 26 ermittelt und resultiert mit einem Wert von $\varepsilon \approx 0,027$.

$$
\varepsilon=\frac{1}{\frac{1}{\varepsilon_{\mathrm{W}}}+\frac{A_{\mathrm{W}}}{A_{\mathrm{O}}} \cdot\left(\frac{1}{\varepsilon_{\mathrm{O}}}-1\right)}
$$

Der berechnete Anteil der Strahlungswärmeabgabe variiert folglich in Abhängigkeit der Übertemperatur der beheizten Platte. Bei einer Oberflächentemperatur der strömungsmechanischen Abschirmung von $\vartheta_{\mathrm{O}}=20^{\circ} \mathrm{C}$ resultiert für eine Plattentemperatur von $\vartheta_{\mathrm{W}}=40^{\circ} \mathrm{C}$ ein spezifischer Strahlungswärmestrom von ca. $\dot{q}_{\mathrm{r}}=3,4 \mathrm{~W} / \mathrm{m}^{2}$; bei $\vartheta_{\mathrm{W}}=90^{\circ} \mathrm{C}$ sind es etwa $\dot{q}_{\mathrm{r}}=15,1 \mathrm{~W} / \mathrm{m}^{2}$. Im Vorgriff auf Kapitel 3.2.3 entspricht dies einem Anteil der Wärmestrahlung an der Gesamtwärmeabgabe der beheizten Platte von $4,3 \%$ bei $\vartheta_{\mathrm{W}}=40^{\circ} \mathrm{C}$ und $3,8 \%$ bei $\vartheta_{\mathrm{W}}=90^{\circ} \mathrm{C}$ (bezogen auf das geometrische Mittel des gesamten Strömungsgebiets, vgl. Kapitel 1.1.1).

\subsubsection{Bestimmung der konvektiven Wärmeübertragung}

Die Bestimmung der konvektiven Wärmeübertragungs-Intensität im stationären Fall $\dot{q}_{c, s t}$ erfolgt auf der Grundlage eines Bilanzierungsansatzes. Dazu wird 
die vorhandene Strahlungswärmeabgabe $\dot{q}_{r, s t}$ der Sandwich-Heizelemente von der zugeführten elektrischen Leistung $\dot{q}_{\mathrm{el}, \text { st }}$ subtrahiert, wie in Gleichung 27 dargestellt.

$$
\dot{q}_{\mathrm{c}, \mathrm{st}}=\dot{q}_{\mathrm{el}, \mathrm{st}}-\dot{q}_{\mathrm{r}, \mathrm{st}}
$$

Gemäß Kapitel 1.1 resultieren der konvektive Wärmeübergangskoeffizient des stationären Falls $\alpha_{c, s t}$ schließlich nach Gleichung 2 und die dimensionsfreie konvektive Wärmeübertragungs-Intensität $N u$ gemäß Gleichung 3.

\subsubsection{Isotherme Oberfläche}

Die im stationären Fall gemessenen konvektiven WärmeübertragungsIntensitäten der isotherm beheizten Platte sind in Abbildung 3.11 als lokale Einzelwerte $N u_{\mathrm{x}, \mathrm{st}}$ und in Abbildung 3.12 für quasi-globale Teilbereiche $N u_{\mathrm{x} / \mathrm{L} \text {,st }}$ (vgl. Kapitel 1.1.1) angegeben.

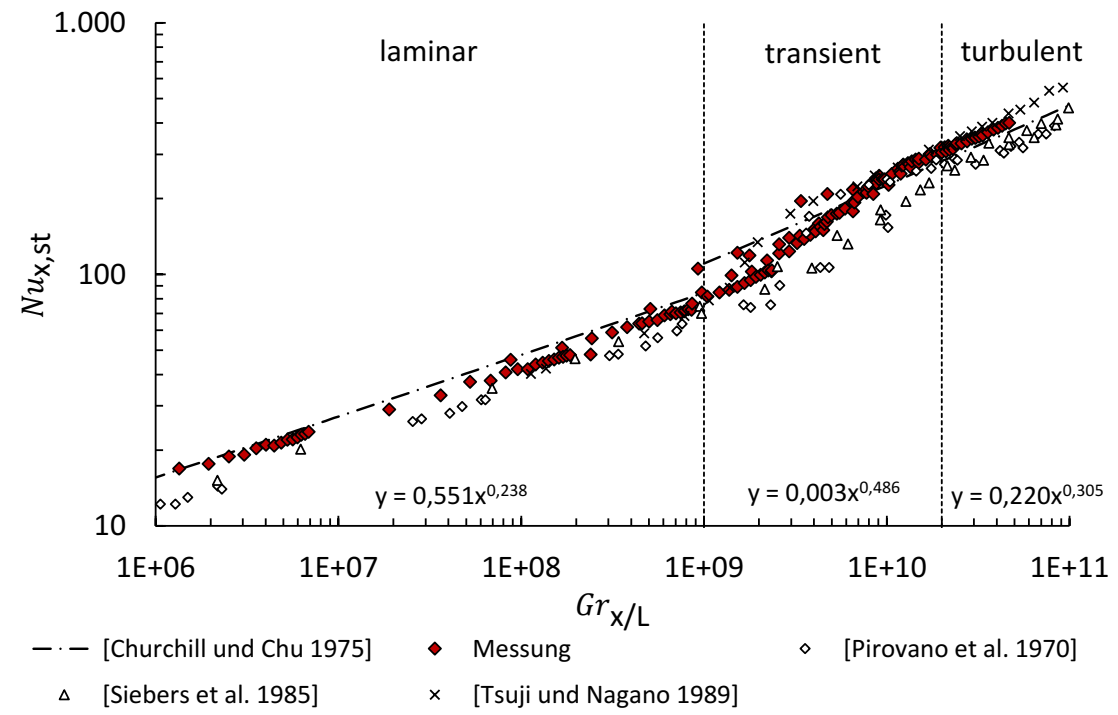

Abbildung 3.11: Lokale Nußelt-Zahlen der isothermen Platte (stationär)

In beiden Fällen ist eine sehr gute Übereinstimmung mit den in der Literatur verfügbaren Vergleichsdaten festzustellen. Auch die empirischen Korrelationen 
gemäß der Gleichungen 8 und 9 zeigen eine gute Kongruenz zu den hier erhobenen Messdaten. Darüber hinaus bestätigen Regressions-Funktionen in Form von Potenzgleichungen die charakteristischen Exponenten in der Größenordnung von etwa $1 / 4$ im laminaren und $1 / 3$ im vollturbulenten Bereich (vgl. Kapitel 1.1).

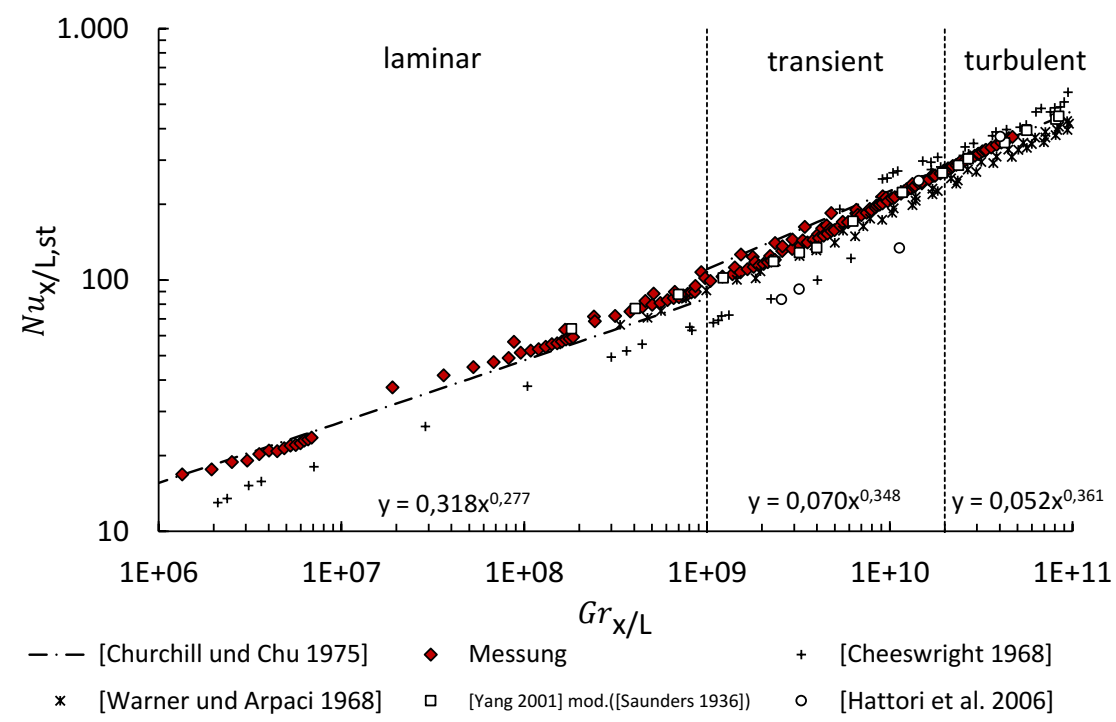

Abbildung 3.12: Quasi-globale Nußelt-Zahlen der isothermen Platte (stationär)

Wie aus Abbildung 3.13 ersichtlich wird, tritt die größte konvektive Wärmestromdichte $\dot{q}_{\mathrm{c}, \mathrm{st}}$ am untersten der zehn Sandwich-Heizelemente auf, da dort der konvektive Wärmeübergang $\alpha_{c, s t}$ aufgrund der Anströmung mit kaltem Umgebungsfluid am größten ist. Mit zunehmender Strömungsweglänge $x / L$ reduziert sich die Intensität der konvektiven Wärmeabfuhr $\dot{q}_{c, s t}$ zunächst deutlich, da die Grenzschicht der betreffenden Bereiche nun mit bereits erwärmtem Fluid durchströmt wird. Im oberen Bereich der beheizten Platte bewirkt die zunehmend turbulente Strömung hingegen eine verstärkte Durchmischung der Grenzschicht und somit eine Induktion von kühlerem Umgebungsfluid, weshalb die konvektive Wärmeabfuhr $\dot{q}_{c, s t}$ dort wieder zunimmt. Die lokalen Gradienten der konvektiven Wärmeübertragung $\partial \dot{q}_{\mathrm{c}, \mathrm{st}} / \partial x$ nehmen dabei mit steigender Temperaturdifferenz $\Delta \vartheta_{\mathrm{W}-\infty} \mathrm{zu}$. 


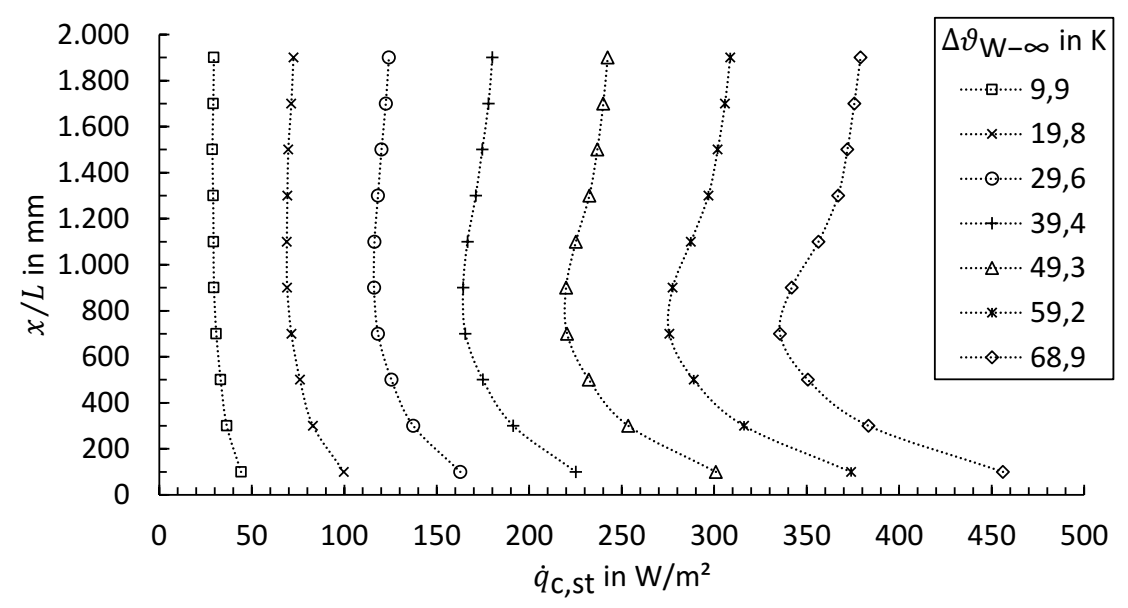

Abbildung 3.13: Lokale Variation der konvektiven Wärmestromdichte (isotherm, stationär)

\subsubsection{Homogene Wärmestromdichte}

Ergänzend zur Regelung der Heizelemente auf eine einheitliche Oberflächentemperatur wird die stationäre konvektive Wärmeübertragung für den Fall einer homogenen Leistungszufuhr $\dot{q}_{\mathrm{el}, \mathrm{st}}$ in sämtlichen Heizelementen betrachtet. Dabei zeigt sich sowohl für lokale Messwerte $N u_{x, s t}$ (Abbildung 3.14), als auch für den quasi-globalen Betrachtungsansatz $N u_{\mathrm{x} / \mathrm{L}, \mathrm{st}}$ (Abbildung 3.15) eine ähnlich gute Übereinstimmung mit Literaturdaten, wie im isothermen Fall.

Die Variation der Oberflächen-Übertemperatur $\Delta \vartheta_{\mathrm{W}-\infty}$ bei homogener Leistungszufuhr $\dot{q}_{\mathrm{el}}$ ist in Abbildung 3.16 für die resultierende konvektive Wärmestromdichte $\dot{q}_{\mathrm{c}, \mathrm{st}}$ nach Gleichung 27 dargestellt. In Analogie zum isothermen Fall (vgl. Abbildung 3.13) charakterisiert das Maximum der OberflächenÜbertemperatur $\Delta \vartheta_{\mathrm{W}-\infty}$ hier den Bereich mit dem geringsten konvektiven Wärmeübergang $\alpha_{\mathrm{c}, \mathrm{st}}$. Dementsprechend nehmen die lokalen Gradienten der Oberflächen-Übertemperatur $\partial \vartheta_{\mathrm{W}-\infty} / \partial x$ hier mit steigender konvektiver Wärmestromdichte $\Delta \dot{q}_{\mathrm{c}, \mathrm{st}} \mathrm{zu}$.

Der beschriebene Verlauf der lokalen konvektiven Wärmeübertragung steht in guter Übereinstimmung mit vorhergehenden Untersuchungen, wie z.B. [Warner und Arpaci 1968], [Pirovano et al 1970] sowie [Miyamoto und Okayama 1982]. 


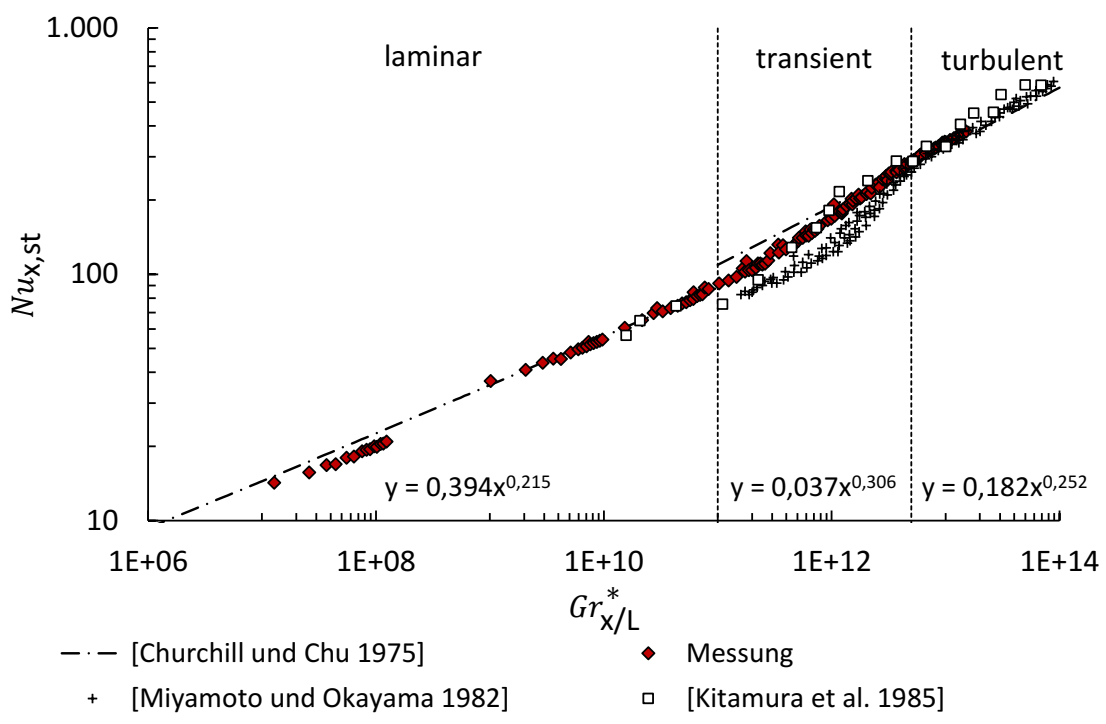

Abbildung 3.14: Lokale Nußelt-Zahlen bei homogener Wärmestromdichte (stationär)

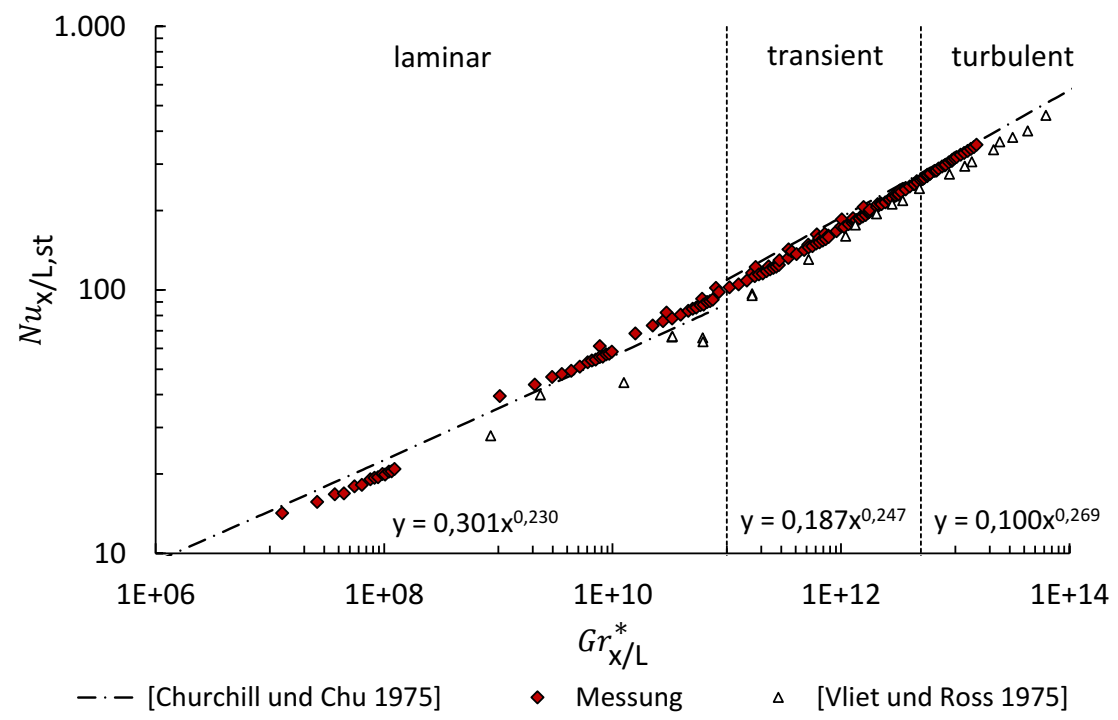

Abbildung 3.15: Quasi-globale Nußelt-Zahlen bei homogener Wärmestromdichte (stationär) 


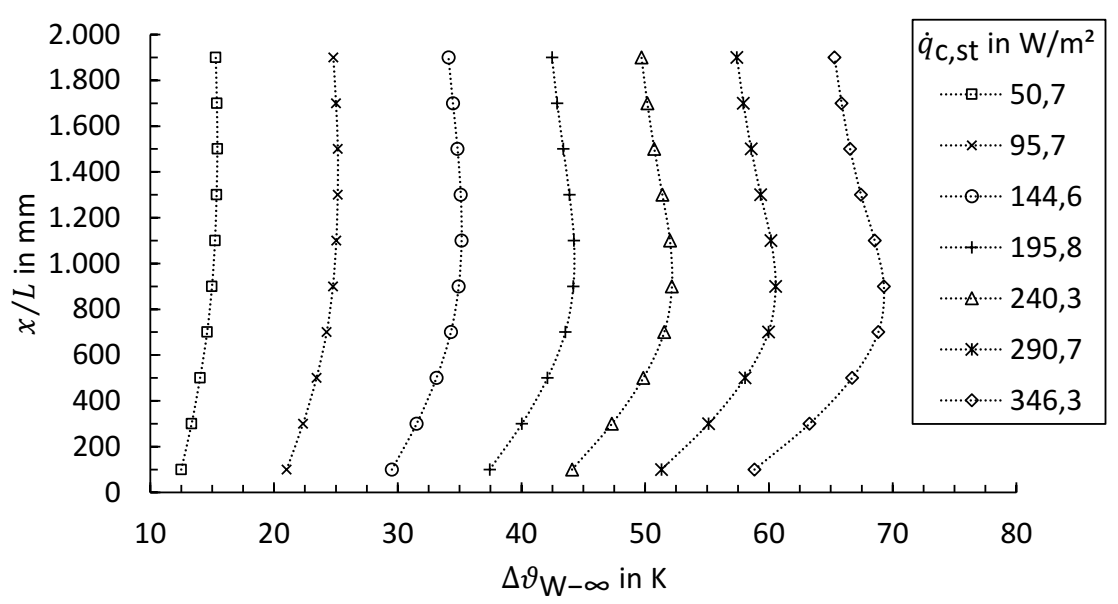

Abbildung 3.16: Lokale Variation der Oberflächentemperatur (homogen, stationär)

\subsection{Instationäre Bewertungsmethodik}

Eine gezielt instationäre Betriebsweise von technischen Einrichtungen zur Übertragung von Wärme durch freie Konvektion wird davon motiviert, die Intensität der konvektiven Wärmeübertragung zu beeinflussen. Für den Fall einer beabsichtigen Intensivierung der Wärmeübertragung zwischen Oberfläche und Umgebungsfluid eröffnen sich im Sinne der Energieerhaltung zwei mögliche Zielpfade:

- eine Reduzierung der mittleren Temperaturen bei gleicher Übertragungsleistung oder

- eine verstärke Übertragungsleistung bei gleichen mittleren Temperaturen.

Konkret bezeichnet eine instationäre Betriebsweise im Folgenden eine Änderung der Randbedingungen, d.h. eine zeitliche Variation der Oberflächentemperatur oder der Wärmestromdichte. Der Fokus der Betrachtungen liegt dementsprechend auf einer Quantifizierung der Auswirkungen solcher Änderungen auf die Intensität der konvektiven Wärmeübertragung. Dies erfolgt anhand eines Vergleichs zwischen einer quasi-stationären und einer instationären Bewertung, wie nachfolgend erläutert. 


\subsubsection{Quasi-stationärer Betrachtungsansatz}

Die quasi-stationäre Vergleichsgrundlage beruht in Analogie zu Kapitel 2.1 auf der Annahme, dass eine im zeitlichen Verlauf veränderliche (instationäre) Strömung zu jedem Zeitpunkt genau die Wärmeübertragungs-Intensität aufweist, die in einer vollständig ausgebildeten (statistisch-stationären) Strömung auftreten würde, in der die Randbedingungen des aktuellen Zeitpunkts dauerhaft vorliegen. Dementsprechend wird bei der quasi-stationären Bewertung einer instationären Versuchsreihe für jeden gemessenen Temperaturdifferenzwert $\Delta \vartheta_{\mathrm{W}-\infty}$ (Messintervall $2 \mathrm{~s}$, vgl. Kapitel 3.1.4) die zugehörige konvektive Wärmestromdichte des stationären Falls $\dot{q}_{c, s t}$ berechnet.

Die Berechnung dieser quasi-stationären Momentanwerte $\dot{q}_{c, s t}$ erfolgt für jeden Strömungs-Teilbereich $x / L$ mit Hilfe eines individuellen Regressions-Polynoms auf Basis der in Kapitel 3.2 dargestellten stationären Messdaten. In Abbildung 3.17 ist dies beispielhaft für zwei Teilbereiche des Strömungsgebiets dargestellt. Die verwendeten Regressions-Polynome dritten Grades repräsentieren die stationären Betriebspunkte des Versuchsaufbaus dabei mit sehr guter Übereinstimmung.

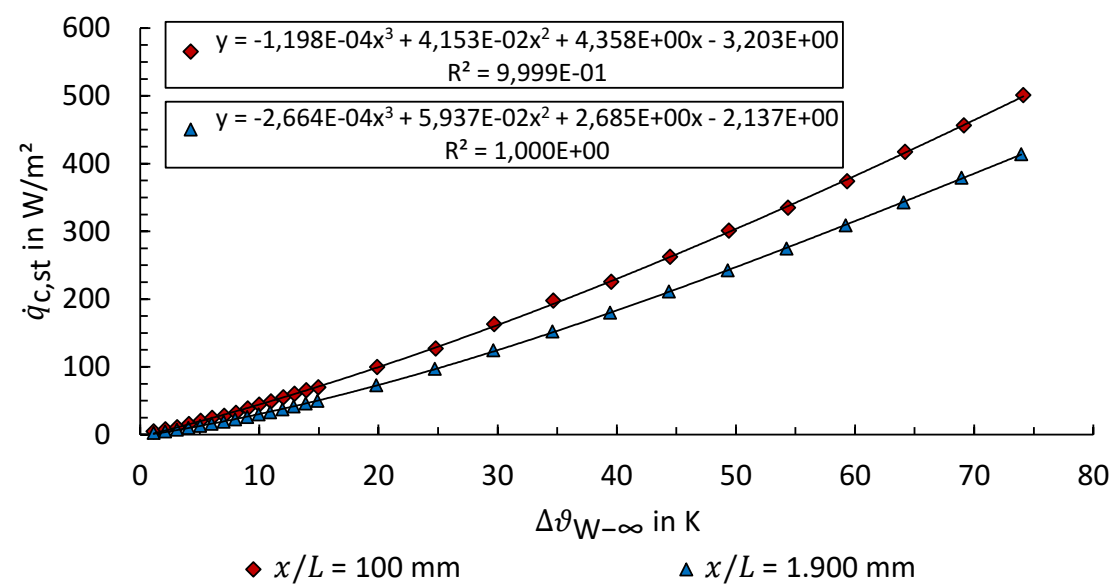

Abbildung 3.17: Regression der konvektiven Wärmestromdichte im stationären Fall

Entsprechend der in Kapitel 1.1.1 beschriebenen Konvention handelt es sich bei der verwendeten Oberflächen-Übertemperatur $\Delta \vartheta_{\mathrm{W}-\infty}$ und der konvektiven 
Wärmestromdichte $\dot{q}_{\mathrm{c}, \mathrm{st}}$ um quasi-globale Mittelwerte des jeweiligen Strömungs-Teilgebiets $x / L$. Diese Betrachtung auf der Grundlage einer räumlichen Mittelung begründet sich primär durch eine Egalisierung der Randbedingungsart. So ist die Korrelation zwischen der mittleren Platten-Übertemperatur und der mittleren konvektiven Wärmestromdichte sowohl für den Fall einer isothermen Platten-Oberfläche, als auch bei einer homogenen Wärmestromdichte identisch, wie Abbildung 3.18 für einen transienten Teilbereich des Strömungsgebiets zeigt.

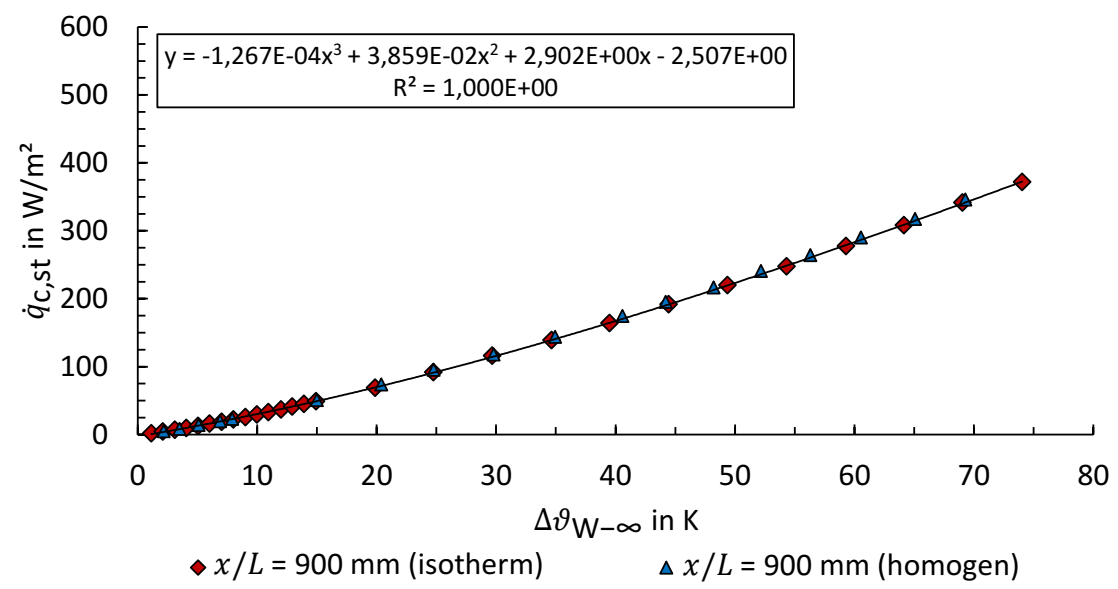

Abbildung 3.18: Quasi-globale Mittelung bei unterschiedlichen Randbedingungen

Die quasi-stationären Momentanwerte $\dot{q}_{\mathrm{c}, \mathrm{st}}$ werden schließlich durch eine zeitliche und temperaturdifferenz-bezogene Mittelung zusammengefasst (Gleichung 28).

$$
\bar{\alpha}_{\mathrm{c}, \mathrm{st}}=\frac{\int \dot{q}_{\mathrm{c}, \mathrm{s}}(t) d t}{\int\left[\vartheta_{\mathrm{W}}(t)-\vartheta_{\infty}(t)\right] d t}
$$

Der resultierende Vergleichswert $\bar{\alpha}_{c, s t}$ repräsentiert folglich den mittleren konvektiven Wärmeübergangskoeffizienten eines Betriebsintervalls mit beliebigen zeitlichen Variationen der Oberflächen-Übertemperatur $\Delta \vartheta_{\mathrm{W}-\infty}$ unter der Annahme, dass die Intensität der konvektiven Wärmeübertragung ein vollständig quasi-stationäres Verhalten aufweist. 


\subsubsection{Bewertung der instationären Wärmeübertragung}

Die Ermittlung der konvektiven Wärmeübertragungs-Intensität, in der auch mögliche Auswirkungen einer instationären Betriebsweise berücksichtigt werden, erfolgt durch eine rein messtechnische Erfassung der relevanten Bilanzgrößen des jeweiligen Betrachtungsintervalls. Dabei ist jedoch zu beachten, dass die Messgrößen aufgrund von thermischen Kapazitäten im instationären Fall zeitlich verschoben sind. So erfolgt beispielsweise auch nach einem Abschalten der elektrischen Leistungszufuhr weiterhin eine konvektive Abgabe der in den Sandwich-Heizelementen gespeicherten Wärme. Folglich ist bei der Bilanzierung der instationären konvektiven Wärmestromdichte $\dot{q}_{c, \text { is }}$ eine teilweise Be- oder Entladung der thermischen Eigenkapazität der wärmeübertragenden Oberfläche $C_{\mathrm{W}}$ zu berücksichtigen, was durch eine Erweiterung der Bilanzgleichung 27 um einen Speicherterm gemäß Gleichung 29 gelingt.

$$
\dot{q}_{\mathrm{c}, \mathrm{is}}=\dot{q}_{\mathrm{el}}-\dot{q}_{\mathrm{r}}-\frac{\partial \vartheta_{\mathrm{w}}}{\partial t} \cdot \frac{C_{\mathrm{W}}}{A_{\mathrm{W}}}
$$

Die messtechnische Erfassung der erforderlichen Momentanwerte der Bilanzgrößen in Gleichung 29 erfolgt durch eine Auswertung der jeweiligen Messgrößen in möglichst kleinen Zeitabschnitten $\Delta t$. Dabei wird die Änderung der gemessenen Oberflächentemperatur innerhalb eines solchen Bilanz-Zeitschritts $\Delta \vartheta_{\mathrm{W}} / \Delta t$ aufgrund der in Kapitel 3.1.5 beschriebenen sehr geringen Reaktionszeit des Versuchsaufbaus und der als homogen angenommenen Temperaturverteilung innerhalb der Heizelemente (vgl. Kapitel 3.1.2) als repräsentativ für die Temperaturänderung des gesamten Speicherterms betrachtet. Bei sehr kleinen Bilanz-Zeitschritten $\Delta t$ oder sehr geringen Temperaturänderungen $\Delta \vartheta_{\mathrm{W}}$ ist jedoch unvermeidbar von einer Zunahme der messtechnischen Unsicherheiten auszugehen. Für den beschriebenen Versuchsaufbau resultieren für BilanzZeitschritte $a b \Delta t \geq 90 \mathrm{~s}$ auch für sehr langsame Temperaturänderungen plausible Momentanwerte der konvektiven Wärmeübertragung $\dot{q}_{c, \text { is }}$ (vgl. Kapitel 3.4). Der Kapazitätsterm aus Gleichung 29 wird ferner ab einer Temperaturänderung von $\Delta \vartheta_{\mathrm{W}} \geq 0,1 \mathrm{~K}$ innerhalb des Bilanz-Zeitschritts von $\Delta t=90 \mathrm{~s}$ berücksichtigt.

Liegt für den Bilanz-Zeitschritt eine Abweichung des instationären Momentanwerts der konvektiven Wärmeübertragung $\dot{q}_{c, \text { is }}$ gemäß Gleichung 29 von dessen 
quasi-stationären Pendant $\dot{q}_{\mathrm{c}, \mathrm{st}}$ nach Gleichung 27 vor, wird dies als Beeinflussung der Intensität der konvektiven Wärmeübertragung durch eine instationäre Betriebsweise interpretiert und gemäß Gleichung 30 als relative Abweichung $\Delta_{\text {is/st }}$ angegeben.

$$
\Delta_{\mathrm{is} / \mathrm{st}}=\frac{\dot{q}_{\mathrm{c}, \mathrm{is}}-\dot{q}_{\mathrm{c}, \mathrm{st}}}{\dot{q}_{\mathrm{c}, \mathrm{st}}}
$$

Beläuft sich die Abweichung $\Delta_{\text {is/st }}$ eines Bilanz-Zeitschritts $\Delta t$ auf mehr als ein Prozent, so wird der Zeitschritt im Folgenden der instationären Phase $\Delta t_{\text {isPh }}$ zugeordnet (Gleichung 31).

$$
\Delta_{\mathrm{is} / \mathrm{st}}(\Delta t) \geq 0,01 \Rightarrow \Delta t \in \Delta t_{\mathrm{isPh}}
$$

Für beliebige Betrachtungszeiträume gelingt eine Quantifizierung des mittleren instationären konvektiven Wärmeübergangs $\bar{\alpha}_{c, \text { is }}$ schließlich in Analogie zu Gleichung 28 durch eine separate zeitliche Integration der Bilanzgrößen gemäß Gleichung 32.

$$
\bar{\alpha}_{\mathrm{c}, \mathrm{is}}=\frac{\int \dot{q}_{\mathrm{e}}(t) d t-\int \dot{q}_{\mathrm{r}}(t) d t-\int \frac{\partial \vartheta_{\mathrm{W}}}{\partial t}(t) \cdot \frac{c_{\mathrm{W}}}{A_{\mathrm{W}}} d t}{\int\left[\vartheta_{\mathrm{W}}(t)-\vartheta_{\infty}(t)\right] d t}
$$

Wird der Integrations-Zeitraum so gewählt, dass eine vollständig ausgeglichene Be- und Entladung der thermischen Kapazitäten vorliegt, entfällt darin die Notwendigkeit zur Berücksichtigung des Speicherterms. Dies gelingt beispielsweise bei der Bilanzierung einer vollständigen Periode von zyklischen Änderungen.

In Analogie zu Gleichung 30 kann die mittlere relative Abweichung der instationären Wärmeübertragung von der quasi-stationären Vergleichsgrundlage $\bar{\Delta}_{\text {is/st }}$ für beliebige Betrachtungszeiträume durch eine zeitliche Integration der jeweiligen Momentanwerte der konvektiven Wärmeübertragung $\dot{q}_{c, i s}$ und $\dot{q}_{c, s t}$ oder durch eine Auswertung der mittleren konvektiven Wärmeübergangskoeffizienten $\bar{\alpha}_{\mathrm{c}, \text { is }}$ und $\bar{\alpha}_{\mathrm{c}, \mathrm{st}}$ erfolgen, wie in Gleichung 33 dargestellt.

$$
\bar{\Delta}_{\mathrm{is} / \mathrm{st}}=\frac{\int \dot{q}_{\mathrm{c}, \mathrm{s} s}(t) d t-\int \dot{q}_{\mathrm{cst}}(t) d t}{\int \dot{q}_{\mathrm{c}, \mathrm{st}}(t) d t}=\frac{\bar{\alpha}_{\mathrm{c}, \mathrm{si}}-\bar{\alpha}_{\mathrm{c}, \mathrm{st}}}{\bar{\alpha}_{\mathrm{c}, \mathrm{st}}}
$$


Der Strahlungswärmeaustausch zwischen den beteiligten Oberflächen wird unter Vernachlässigung der Strahlungsabsorption von feuchter Luft als unabhängig vom Umgebungsfluid und von dessen Strömungsstrukturen betrachtet. Die Berücksichtigung der Wärmestrahlung gemäß Kapitel 3.2.1 erfolgt daher in Analogie zu Kapitel 3.3.1 auch für veränderliche Randbedingungen stets auf der Grundlage eines quasi-stationären Betrachtungsansatzes.

\subsubsection{Thermische Kapazität der Sandwich-Heizelemente}

Zur Bestimmung von Momentanwerten der instationären konvektiven Wärmeübertragung gemäß Kapitel 3.3.2 ist die Kenntnis der thermischen Kapazität der Sandwich-Heizelemente $C_{\mathrm{W}}$ erforderlich. Für das Trägermaterial der Heizleiterbahnen liegen jedoch aufgrund eines Werkstoffverbundes (Silikon-Glasgewebe, vgl. Kapitel 3.1.2) keine gesicherten Stoffdaten zur spezifischen Wärmekapazität vor. Aus einer Abschätzung auf der Grundlage von Stoffwerten für reine Silikon-Elastomere $(90 \%)$ und reines Glas $(10 \%)$ resultiert eine spezifische Wärmekapazität von ca. $1.400 \mathrm{~J} /(\mathrm{kg} \mathrm{K})$, welche gut mit Angaben für glasfaserverstärkte Kunststoffe übereinstimmt. Für den in Kapitel 3.1.2 beschriebenen Aufbau berechnet sich die Gesamtkapazität der zehn Heizelemente somit wie in Tabelle 3.2 dargestellt.

Tabelle 3.2: Thermische Gesamtkapazität der zehn Sandwich-Heizelemente

\begin{tabular}{l|cccc} 
Schicht & $\begin{array}{c}\mathbf{V} \\
\text { in } \mathbf{~ m}^{\mathbf{3}}\end{array}$ & $\begin{array}{c}\boldsymbol{\rho} \\
\text { in } \mathbf{~ k g} / \mathbf{m}^{\mathbf{3}}\end{array}$ & $\begin{array}{c}\mathbf{c} \\
\text { in J/(kg K) }\end{array}$ & $\begin{array}{c}\mathbf{C} \\
\text { in J/K }\end{array}$ \\
\hline $\begin{array}{l}2 \times 0,7 \text { mm Kupfer } \\
\text { [Cu-DHP_CW024A] }\end{array}$ & $28 \cdot 10^{-4}$ & 8.940 & 389,5 & 9.750 \\
$\begin{array}{l}1,1 \text { mm Silikon- } \\
\text { Glasgewebe [kSil }\end{array}$ & $21,45 \cdot 10^{-4}$ FA60] & 1.250 & $\begin{array}{c}1.400 \\
\text { (Annahme) }\end{array}$ & 3.754 \\
$\begin{array}{l}\text { 0,05 mm Leiterbahnen, } \\
\text { Belegung ca. 55\% } \\
\text { [AK Steel 18 SR }{ }^{\mathrm{TM}} \text { ] }\end{array}$ & $55 \cdot 10^{-6}$ & 7.450 & 500 & 205 \\
\hline $\begin{array}{l}\text { Summe } \\
\text { Summe }\end{array}$ & & & & $\mathbf{1 3 . 7 0 9}$
\end{tabular}

Darüber hinaus wird im Rahmen einer Versuchsreihe die tatsächlich vorhandene Wärmekapazität näherungsweise messtechnisch ermittelt. Dem liegt die Annahme zugrunde, dass bei hinreichend langsamen Temperaturänderungen 
keine instationären Effekte zu erwarten sind und die konvektive Wärmeabfuhr zu jedem Zeitpunkt als quasi-stationär betrachtet werden kann. Sämtliche Heizelemente werden dazu zeitgleich mit einer Temperaturänderungsrate von $\partial \vartheta_{\mathrm{w}} / \partial t=1 \mathrm{~K} / \mathrm{Min}$ kontrolliert isotherm auf eine Übertemperatur von $\Delta \vartheta_{\mathrm{W}-\infty}=75 \mathrm{~K}$ erwärmt. Dabei wird zu jedem Zeitpunkt des Aufheizvorgangs die Wärmeabfuhr aufgrund von Strahlung $\dot{q}_{\mathrm{r}}$ (vgl. Kapitel 3.2.1) und Konvektion $\dot{q}_{\mathrm{c}, \mathrm{st}}$ (vgl. Kapitel 3.3.1) für den quasi-stationären Vergleichsfall bestimmt und von der zugeführten elektrischen Leistung $\dot{q}_{\text {el }}$ subtrahiert. Die verbleibende Differenz spiegelt in Analogie zu Gleichung 29 folglich die Wärmemenge zur Beladung der thermischen Kapazitäten wider. Abbildung 3.19 zeigt die EnsembleMittel der Gesamt-Wärmekapazität $C_{\mathrm{W}}$ der zehn Heizelemente aus einer fünffach wiederholten Messung für eine Bilanzschrittweite von jeweils $5 \mathrm{~K}$.

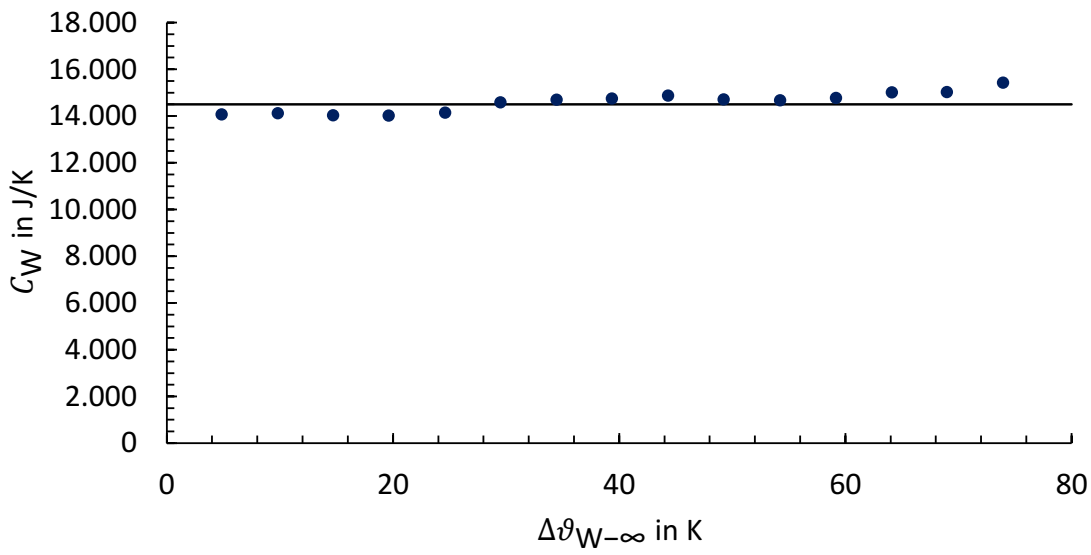

Abbildung 3.19: Gemessene thermische Gesamtkapazität der Heizelemente

Das arithmetische Mittel aller Bilanzabschnitte ergibt sich dabei mit $C_{\mathrm{W}}=14.589 \mathrm{~J} / \mathrm{K}$ bei einer Standardabweichung von $s=415 \mathrm{~J} / \mathrm{K}$. Für eine Bilanz der Wärmemengen im gesamten Aufheizvorgang ( $75 \mathrm{~K}$ ) resultiert eine Wärmekapazität von $C_{\mathrm{W}}=14.508 \mathrm{~J} / \mathrm{K}$. Die messtechnisch ermittelten Werte weichen folglich ca. $6 \%$ von der unter Annahmen berechneten Kapazität ab und bestätigen somit deren Plausibilität. Im Folgenden wird daher die GesamtWärmekapazität der zehn Sandwich-Heizelemente mit einem Wert von $C_{\mathrm{W}}=14.500 \mathrm{~J} / \mathrm{K}$ berücksichtigt. 


\subsubsection{Messunsicherheits-Fortpflanzung}

Mit Hilfe der Angaben zur Genauigkeit der verwendeten Messtechnik (siehe Kapitel 3.1.4) kann die messtechnische Gesamtunsicherheit aufgrund von Fortpflanzungseffekten für die dargestellte Bewertungsmethodik angegeben werden. Diese ist jedoch nicht als konstant zu betrachten, sondern variiert in Abhängigkeit der jeweils vorliegenden Randbedingungen. So beträgt die kombinierte Messunsicherheit aus Sensor und Messwertaufnahme zur Erfassung der Umgebungstemperatur etwa $\pm 0,14 \mathrm{~K}$ bei $\vartheta_{\infty}=20^{\circ} \mathrm{C}$. Für die Oberflächentemperatur der Sandwich-Heizelemente liegt bei $\vartheta_{\mathrm{W}}=40^{\circ} \mathrm{C}$ eine Unsicherheit von ca. $\pm 0,23 \mathrm{~K}$ vor, bei $\vartheta_{\mathrm{W}}=90^{\circ} \mathrm{C}$ sind es $\pm 0,33 \mathrm{~K}$. Temperaturdifferenzen zwischen der beheizten Oberfläche und der Umgebung können folglich mit einer Unsicherheit von ca. $1,9 \%$ bei $\Delta \vartheta_{\mathrm{W}-\infty}=20 \mathrm{~K}$ und $0,7 \%$ bei $\Delta \vartheta_{\mathrm{W}-\infty}=70 \mathrm{~K}$ erfasst werden. Die Messunsicherheit der Leistungszufuhr liegt nach Kalibrierung (vgl. Kapitel 3.1.3) bei ca. 1,4 \% für eine Wärmestromdichte von $\dot{q}_{\mathrm{el}}=98 \mathrm{~W} / \mathrm{m}^{2}$ und bei rund 0,6 \% für eine Wärmestromdichte von $\dot{q}_{\mathrm{el}}=1.418 \mathrm{~W} / \mathrm{m}^{2}$.

Die messtechnische Gesamtunsicherheit für Angaben zur Abweichung der Intensität der konvektiven Wärmeübertragung des instationären Falls gegenüber der quasi-stationären Betrachtung $\bar{\Delta}_{\text {is/st }}$ gemäß Gleichung 33 bewegt sich somit in einer Größenordnung von ca. 0,9 \% bis 2,4\%.

\subsection{Versuchsreihen mit veränderlichen Randbedingungen}

Die in Kapitel 3.3 beschriebenen Methoden zur Quantifizierung eines möglichen Einflusses von instationären Strömungsphänomenen auf die konvektive Wärmeübertragung werden nachfolgend zur Bewertung verschiedenartiger Änderungen der Strömungs-Randbedingungen herangezogen.

\subsubsection{Zyklische Variationen}

Im Fokus der experimentellen Untersuchungen stehen zyklische Variationen der Wärmestromzufuhr mit variablen Frequenzen und Amplituden. Dazu werden zunächst 52 Versuchsreihen mit einer möglichst großen Bandbreite von Variations-Randbedingungen durchgeführt, die entsprechend der in Kapitel 1.1.1 getroffenen Konvention zur Bilanzierung von Teilabschnitten des Strömungsgebiets und zehn messtechnisch separat erfassten Sandwich-Heizele- 
menten (vgl. Kapitel 3.1) zu einer Stichprobe von insgesamt 520 Betrachtungsfällen führen. Der prinzipielle Versuchs-Ablauf ist jedoch für alle zyklischen Variationen identisch. So wird den Heizelementen zunächst für eine definierte Dauer $\Delta t_{\text {Impuls }}$ eine konstante und homogene Leistung $\dot{q}_{\text {el }}$ aufgeprägt. Im Anschluss erfolgt für einen ebenfalls festgelegten Zeitabschnitt $\Delta t_{\text {Auskühlung }}$ eine Auskühlung ohne aktive Zufuhr von Wärme. Diese Abfolge wird im Rahmen einer Versuchsreihe so lange wiederholt, bis im Vergleich zum vorherigen Zyklus keine Änderungen der minimalen, maximalen und mittleren Größen mehr feststellbar sind.

Zur eindeutigen Charakterisierung der instationären Betriebsweise wird für die zyklischen Variationen anstelle der elektrischen Leistungszufuhr $\dot{q}_{\text {el }}$ die ImpulsWärmestromdichte $\dot{q}_{\text {Impuls }}$ als Randbedingung angegeben, die gemäß Gleichung 34 eine um die Strahlungswärmeabgabe $\dot{q}_{\mathrm{r}}$ bereinigte Leistungszufuhr repräsentiert.

$$
\dot{q}_{\text {Impuls }}=\dot{q}_{\mathrm{el}}-\dot{q}_{\mathrm{r}}
$$

Für den stationären Fall entspricht $\dot{q}_{\text {Impuls }}$ folglich der konvektiven Wärmestromdichte $\dot{q}_{c, s t}$ (vgl. Gleichung 27). Bei einer instationären Betriebsweise wird ein Teil der Impulswärmestromdichte $\dot{q}_{\text {Impuls }}$ jedoch der thermischen Kapazität der beheizten Platte $C_{\mathrm{W}}$ zugeführt (siehe Gleichung 29).

Im Rahmen der 52 Versuchsreihen werden Impuls-Wärmestromdichten in einem Bereich von $57 \mathrm{~W} / \mathrm{m}^{2} \leq \dot{q}_{\text {Impuls }} \leq 1.430 \mathrm{~W} / \mathrm{m}^{2}$ aufgeprägt. Die Zyklendauer variiert von $510 \mathrm{~s} \leq \Delta t_{\text {Zyklus }} \leq 2.700 \mathrm{~s}$ und beinhaltet Impulszeiten von $15 \mathrm{~s} \leq$ $\Delta t_{\text {Impuls }} \leq 1.800$ s. Die zeitlichen Mittelwerte der Grashof-Zahlen liegen dabei im Bereich $1,1 \cdot 10^{8}<\overline{G r}_{\text {x/L,Zyklus }}^{*}<1,7 \cdot 10^{13}$.

Ein Vergleich zwischen der gemessenen mittleren WärmeübertragungsIntensität der Zyklen und der quasi-stationären Betrachtungsweise zeigt für die durchgeführten Versuchsreihen teils deutliche Unterschiede. In Abbildung 3.20 ist dazu eine Übersicht der mittleren prozentualen Abweichung $\bar{\Delta}_{\mathrm{is} / \mathrm{st}}$ gemäß Gleichung 33 in Abhängigkeit des zeitlichen Mittelwerts der Grashof-Zahl des jeweiligen Zyklus $\overline{G r}_{\text {x/L,Zyklus }}^{*}$ dargestellt. Die Unterschiede belaufen sich, bezogen auf die Gesamtdauer der jeweiligen Zyklen, im Maximum auf ca. $\bar{\Delta}_{\text {is/st }}=9 \%$. Festzustellen ist dabei insbesondere eine ausgeprägte Abhängigkeit 
der mittleren Abweichung $\bar{\Delta}_{\text {is/st }}$ vom zeitlichen Mittel der Grashof-Zahl

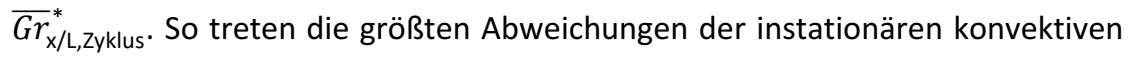
Wärmeübertragung vom quasi-stationären Vergleichsfall $\bar{\Delta}_{\text {is/st }}$ im Bereich um $\overline{G r}_{\mathrm{x} / \mathrm{L}, \mathrm{zyklus}}^{*} \approx 10^{11}$ auf, also zu Beginn des laminar-turbulenten Transitionsbereiches.

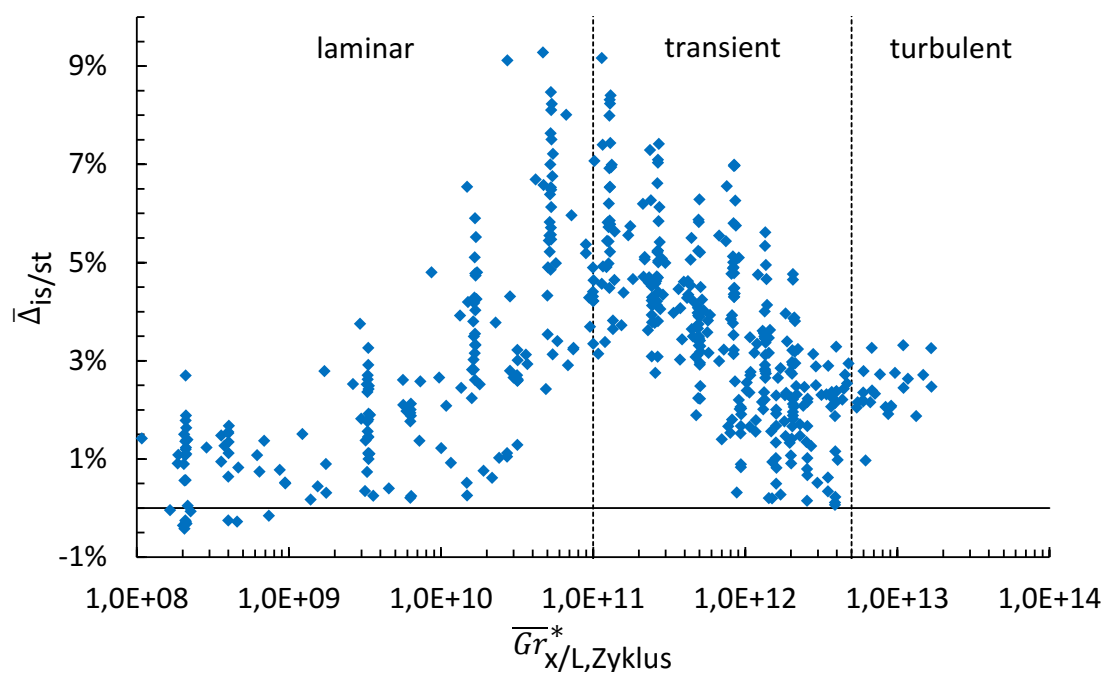

Abbildung 3.20: Zusätzliche konvektive Wärmeübertragung bei zyklischen Variationen

Mit zunehmender Entfernung der mittleren Grashof-Zahl von diesem kritischen Wert zeigt sich sowohl im laminaren Strömungsgebiet, als auch im transienten und vollturbulenten Bereich eine Tendenz zu einer weniger starken Intensivierung der konvektiven Wärmeübertragung durch die instationäre Betriebsweise. Die Bandbreite, in der der Unterschied zwischen der quasi-stationären Betrachtung und der tatsächlichen instationären Wärmeübertragung $\bar{\Delta}_{\text {is/st }}$ bei gleicher mittlerer Grashof-Zahl variiert, verdeutlicht jedoch eine zusätzliche Relevanz weiterer Einflussgrößen.

Ergänzend zur Analyse von zeitlichen Mittelwerten sind in Abbildung 3.21 beispielhaft die Momentanwert-Verläufe der Oberflächentemperatur $\vartheta_{\mathrm{W}}$ und der Umgebungstemperatur $\vartheta_{\infty}$ als geometrische Mittelwerte des Strömungs- 
Teilgebiets $x / L=1.900 \mathrm{~mm}$ (vgl. Kapitel 1.1.1) sowie des zugeführten Wärmestroms $\dot{q}_{\text {Impuls }}$ einer zyklischen Variation aufgetragen.

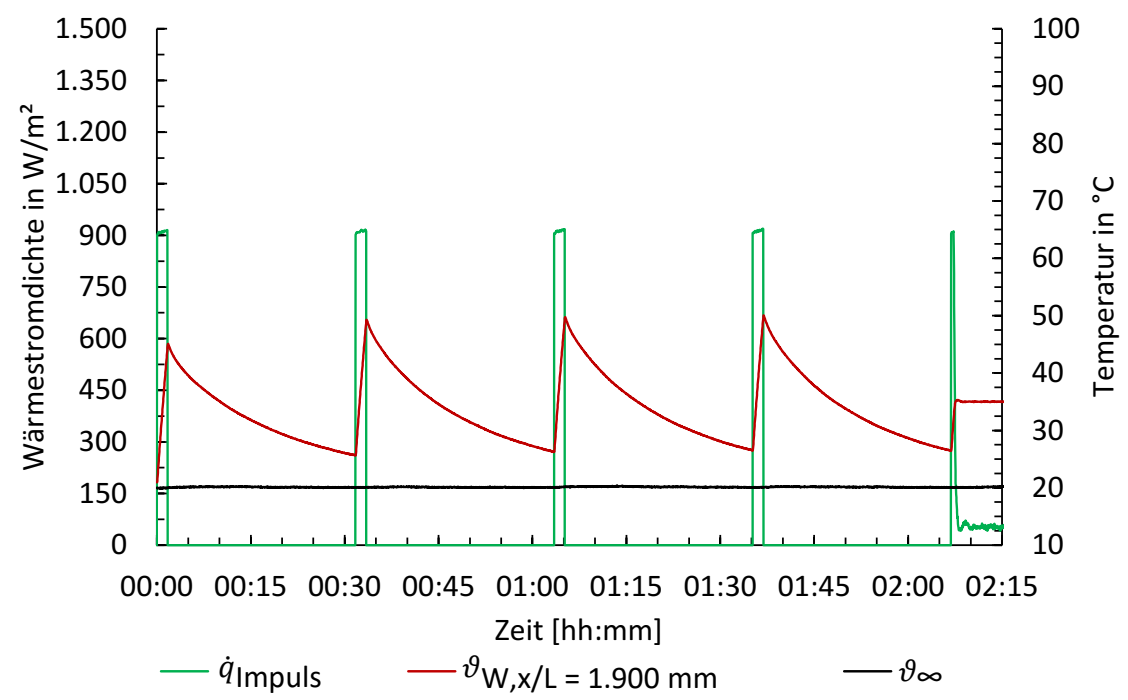

Abbildung 3.21: Temperatur- und Leistungsverlauf bei zyklischen Variationen (mit $\dot{q}_{\text {Impuls }}=913 \mathrm{~W} / \mathrm{m}^{2}, \Delta t_{\text {Impuls }}=102 \mathrm{~s} \mathrm{und} \Delta t_{\text {Auskühlung }}=1.800 \mathrm{~s}$ )

In der dargestellten Versuchsreihe wird für eine Impulsdauer von $\Delta t_{\text {Impuls }}=102 \mathrm{~s}$ eine Wärmestromdichte von $\dot{q}_{\text {Impuls }}=913 \mathrm{~W} / \mathrm{m}^{2}$ aufgeprägt. Im Anschluss folgt eine Auskühlphase von $\Delta t_{\text {Auskühlung }}=1.800 \mathrm{~s}$, wodurch sich gemäß Gleichung 35 eine auf die Gesamtdauer des Zyklus von $\Delta t_{\text {Zyklus }}=1.902 \mathrm{~s}$ bezogene mittlere Wärmestromdichte von etwa $\overline{\dot{q}}_{\text {Zyklus }}=49 \mathrm{~W} / \mathrm{m}^{2}$ ergibt.

$$
\overline{\dot{q}}_{\text {Zyklus }}=\frac{\dot{q}_{\text {Impuls }} \cdot \Delta t_{\text {Impuls }}}{\Delta t_{\text {Zyklus }}}
$$

Betrachtet wird der vierte Zyklus (ca. 01:35 bis 02:07), da die erfassten Messgrößen für diesen Zyklus erstmals identisch zum vorherigen sind. Die Temperaturdifferenz zwischen Wandoberfläche und Umgebungsfluid $\Delta \vartheta_{\mathrm{W}-\infty}$ bewegt sich dabei bezogen auf das geometrische Mittel des gesamten Strömungsgebiets zwischen 6,4 K und $30 \mathrm{~K}$; das zeitliche Mittel liegt bei 14,3 K. Die zugehörigen Grashof-Zahlen variieren zwischen $1,1 \cdot 10^{8}<G r_{x / L}^{*}<9,4 \cdot 10^{8}$ am untersten 
Heizelement $(x / L=100 \mathrm{~mm})$ und $1,4 \cdot 10^{12}<G r_{\mathrm{x} / L}^{*}<9,1 \cdot 10^{12}$ an der obersten der zehn Platten $(x / L=1.900 \mathrm{~mm})$. Die zeitlichen Mittelwerte der GrashofZahlen dieser Versuchsreihe liegen im Bereich $4 \cdot 10^{8}<\overline{G r}_{x / L, \text { Zyklus }}^{*}<3,9 \cdot 10^{12}$. Abbildung 3.22 zeigt den zugehörigen zeitlichen Verlauf der konvektiv von den Heizelementen abgeführten Wärmestromdichte.

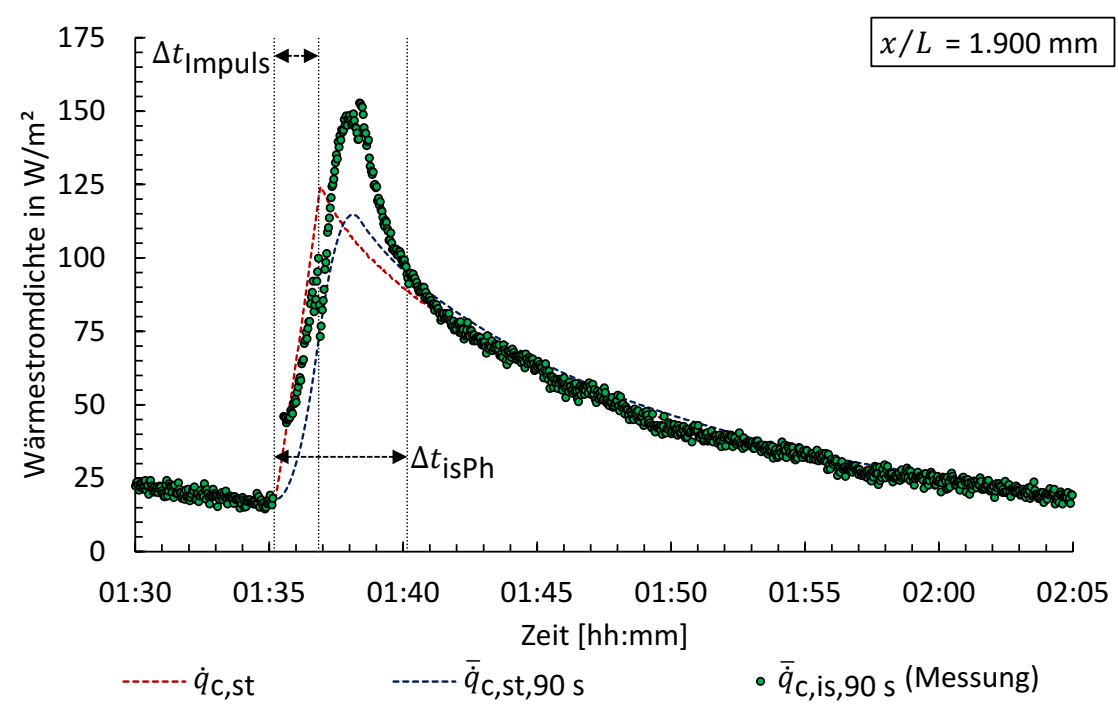

Abbildung 3.22: Konvektive Wärmeübertragung bei zyklischen Variationen (mit $\dot{q}_{\text {Impuls }}=913 \mathrm{~W} / \mathrm{m}^{2}, \Delta t_{\text {Impuls }}=102 \mathrm{~s}$ und $\Delta t_{\text {Auskühlung }}=1.800 \mathrm{~s}$ )

Dargestellt sind in Rot die Werte der quasi-stationären Betrachtung $\dot{q}_{\mathrm{c}, \mathrm{st}}$, wie sie mit Hilfe der in Kapitel 3.3.1 beschriebenen Regressionspolynome für die jeweils aktuelle Temperaturdifferenz $\Delta \vartheta_{\mathrm{W}-\infty}$ eines Zeitpunkts resultieren. Aufgrund der in Kapitel 3.3.2 erläuterten Notwendigkeit zur Bilanzierung eines Zeitintervalls von min. $90 \mathrm{~s}$ bei der messtechnischen Erfassung der grün dargestellten Momentanwerte $\overline{\dot{q}}_{\mathrm{c}, \mathrm{is}, 90 \mathrm{~s}}$ wird in Blau zusätzlich der gleitende Mittelwert der letzten 90 s für die quasi-stationäre Vergleichsgrundlage $\overline{\dot{q}}_{c, s t, 90 \text { s }}$ angegeben. Dabei ist für die im instationären Fall gemessenen Momentanwerte $\overline{\dot{q}}_{\mathrm{c}, \mathrm{s}, 90 \mathrm{~s}}$ insbesondere während des Wärmestrom-Impulses sowie einige Zeit danach eine deutlich intensivere konvektive Wärmeabfuhr zu erkennen, als sie die quasi-stationäre Betrachtung $\overline{\dot{q}}_{\mathrm{c}, \mathrm{st}, 90 \mathrm{~s}}$ erwarten lassen würde. Im weiteren 
Verlauf gleichen sich die gemessenen Momentanwerte $\overline{\dot{q}}_{\mathrm{c}, \mathrm{is}, 90 \mathrm{~s}}$ asymptotisch dem quasi-stationären Niveau $\overline{\dot{q}}_{c, s t, 90 \mathrm{~s}}$ an. Das Maximum der Abweichung zwischen den instationären Messwerten $\overline{\dot{q}}_{\mathrm{c}, \mathrm{is}, 90 \mathrm{~s}}$ und der quasi-stationären Betrachtungsweise $\overline{\dot{q}}_{\mathrm{c}, \mathrm{st}, 90 \mathrm{~s}}$ beläuft sich für das dargestellte Strömungsteilgebiet $(x / L=1.900 \mathrm{~mm})$ auf ca. $\Delta_{\mathrm{is} / \mathrm{st}, \max }=30,2 \%$ und tritt zum Ende des Wärmestrom-Impulses auf.

Während des Auskühlvorgangs resultiert am untersten der zehn SandwichHeizelemente zudem eine bilanzielle Unterschreitung der quasi-stationären Vergleichswerte $\overline{\dot{q}}_{\mathrm{c}, \mathrm{st}, 90 \mathrm{~s}}$ durch die gemessenen Momentanwerte $\overline{\dot{q}}_{\mathrm{c}, \mathrm{is}, 90 \mathrm{~s}}$, wie in Abbildung 3.23 ersichtlich wird.

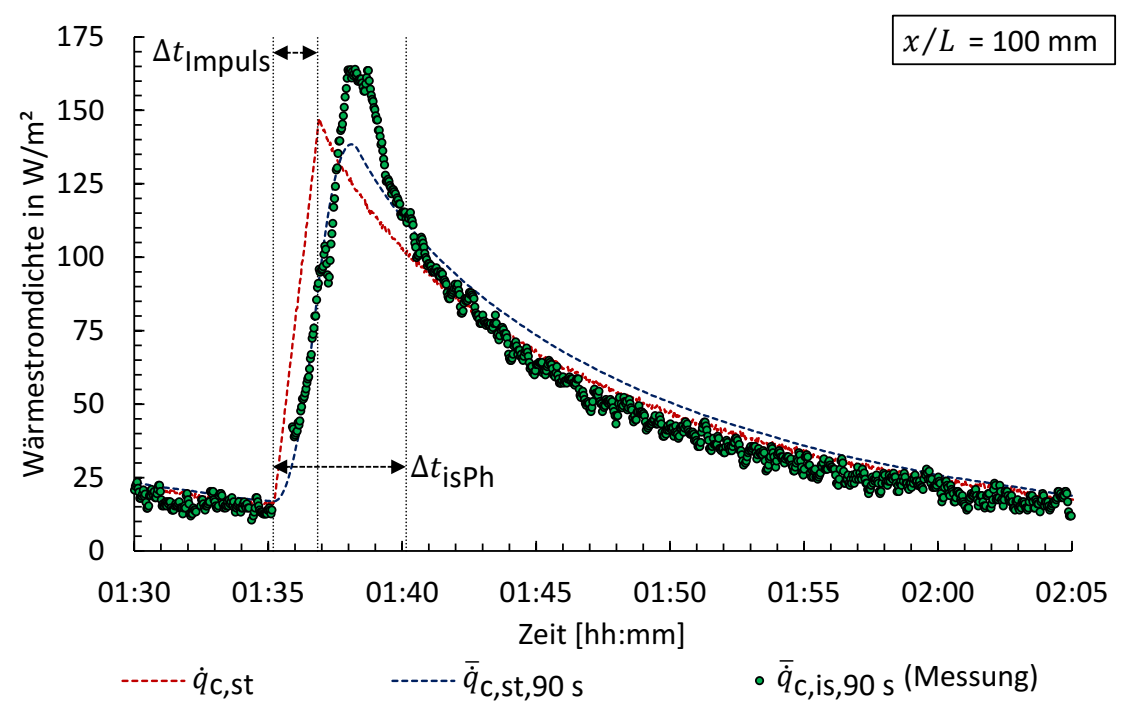

Abbildung 3.23: Konvektive Wärmeübertragung am untersten Element bei zyklischen Variationen (mit $\dot{q}_{\text {Impuls }}=913 \mathrm{~W} / \mathrm{m}^{2}, \Delta t_{\text {Impuls }}=102 \mathrm{~s}$ und $\Delta t_{\text {Auskühlung }}=1.800 \mathrm{~s}$ )

Für die darüber liegenden Heizelemente tritt dieser Effekt nicht auf; lediglich am obersten Heizelement ist ebenfalls ein ähnlicher Verlauf festzustellen, dort jedoch in einer deutlich schwächeren Ausprägung (vgl. auch Abbildung 3.22). In ergänzenden Versuchsreihen ist dasselbe Phänomen auch dann zu beobachten, wenn nur ein beliebiger Teilbereich der zehn Heizelemente einer zyklischen Variation der Randbedingungen unterzogen wird. So ist für das unterste und 
oberste Element des jeweils beheizten Teilbereichs während des Auskühlvorgangs stets eine geringfügige Unterschreitung des quasi-stationären Vergleichsniveaus $\overline{\dot{q}}_{\mathrm{c}, \mathrm{st}, 90 \mathrm{~s}}$ durch die gemessenen Momentanwerte $\overline{\dot{q}}_{\mathrm{c}, \mathrm{is}, 90 \mathrm{~s}}$ festzustellen. Strömungs-geometrische Ursache, wie z.B. ein möglicher Einfluss der am unteren und oberen Ende der Platte befindlichen Kanten, können somit ausgeschlossen werden. Eine detaillierte Analyse der lokalen Temperaturverteilung in Plattenebene $\partial \vartheta_{\mathrm{w}} / \partial x$ zeigt jedoch, dass während des Auskühlvorgangs in ebendiesen Randbereichen eine nicht vollständig isotherme Oberfläche vorliegt. So ergaben stichprobenartige Messungen mit einem reaktionsschnellen Kontakt-Thermometer während des Auskühlvorgangs in Abhängigkeit des jeweiligen Temperaturniveaus maximale Unterschiede von $1 \mathrm{~K}$ bis $5 \mathrm{~K}$ zwischen der Mitte des untersten Heizelements und dessen unterstem Randbereich. In der Folge wird die Entladung der thermischen Kapazität $C_{\mathrm{W}}$ des untersten Heizelements gemäß Gleichung 29 und somit die lokale konvektive Wärmeabfuhr $\overline{\dot{q}}_{\mathrm{c}, \mathrm{is}, 90 \mathrm{~s}}$ im Auskühlvorgang unterschätzt. Die Wärmemenge, die während der scheinbaren Unterschreitung des quasi-stationären Niveaus $\overline{\dot{q}}_{c, s t, 90 \mathrm{~s}}$ im Auskühlvorgang am untersten Heizelement gemessen wird, entspricht unter Bezug auf die Wärmekapazität $C_{\mathrm{W}}$ des untersten Heizelements im Mittel aller Versuchsreihen einer Abweichung der mittleren Oberflächentemperatur $\vartheta_{\mathrm{W}}$ von rund $3 \mathrm{~K}$ und liegt somit in guter Übereinstimmung mit den stichprobenartig erfassten lokalen Temperaturgradienten $\partial \vartheta_{\mathrm{w}} / \partial x$.

Die Intensität und die räumliche Ausdehnung des nicht-isothermen Bereichs fällt am untersten Heizelement ebenfalls erkennbar größer aus, als es am obersten beheizten Element der Fall ist. Dies ist insbesondere auf den im Anströmbereich (leading-edge) maximalen räumlichen Gradienten der Intensität der konvektiven Wärmeübertragung $\partial \dot{q}_{c} / \partial x$ zurückzuführen (vgl. Abbildung 3.13). Für eine korrekte messtechnische Erfassung der lokalen Momentanwerte der konvektiven Wärmeübertragung $\overline{\dot{q}}_{\mathrm{c}, \mathrm{is}, 90 \mathrm{~s}}$ des Auskühlvorgangs wäre daher im unteren Plattenbereich eine feinere räumlich Diskretisierung der Heizelemente erforderlich. Für die übrigen Heizelemente sind während der Auskühlphase keine lokalen Temperaturunterschiede feststellbar.

Da die Auskühlvorgänge im Vergleich zu den betrachteten Aufheizvorgängen nur sehr langsame zeitliche Änderungen der Oberflächentemperatur aufweisen (siehe Kapitel 5.2.2), wird die konvektive Wärmeübertragung nach der asymp- 
totischen Annäherung der Momentanwerte des Aufheiz-Impulses $\overline{\dot{q}}_{\mathrm{c}, \mathrm{is}, 90 \mathrm{~s}}$ an die quasi-stationäre Vergleichsbasis $\overline{\dot{q}}_{c, s t, 90 \mathrm{~s}}$ im Folgenden als quasi-stationär betrachtet. Für laminare Auftriebsströmungen, wie sie am untersten Heizelement ausschließlich vorliegen, wurden quasi-stationäre Verhältnisse bei hinreichend langsamen Temperaturänderungen der wärmeübertragenden Oberfläche bereits mehrfach experimentell bestätigt (vgl. Kapitel 2.1).

Während des Aufheizvorgangs und im stationären Fall sind an keinem Heizelement örtliche Temperaturgradienten feststellbar, was auf die dann vollflächige Wärmestrom-Zufuhr und eine Homogenisierung durch die Wärmeleitung in der Plattenebene zurückgeführt wird (vgl. Kapitel 3.1.2 und 3.2.3).

Der Zeitraum einer Überschreitung der quasi-stationären Vergleichswerte $\overline{\dot{q}}_{\mathrm{c}, \mathrm{st}, 90 \mathrm{~s}}$ durch die Momentanwerte des instationären Falls $\overline{\dot{q}}_{\mathrm{c}, \mathrm{is}, 90 \mathrm{~s}}$ erstreckt sich in der betrachteten Versuchsreihe auf ca. $16 \%$ der Gesamt-Zyklusdauer $\Delta t_{\text {zyklus. }}$ Gemäß Kapitel 3.3.2 werden hierfür Abweichungen $\Delta_{\mathrm{is} / \mathrm{st}} \geq 1 \%$ berücksichtigt. Mit Bezug auf alle betrachteten zyklischen Versuchsreihen variiert die Dauer dieser instationären Phase $\Delta t_{\text {isph }}$ zwischen $12 \%$ und $51 \%$ der GesamtZyklusdauer $\Delta t_{\text {Zyklus }}$ das arithmetische Mittel liegt bei $25 \%$.

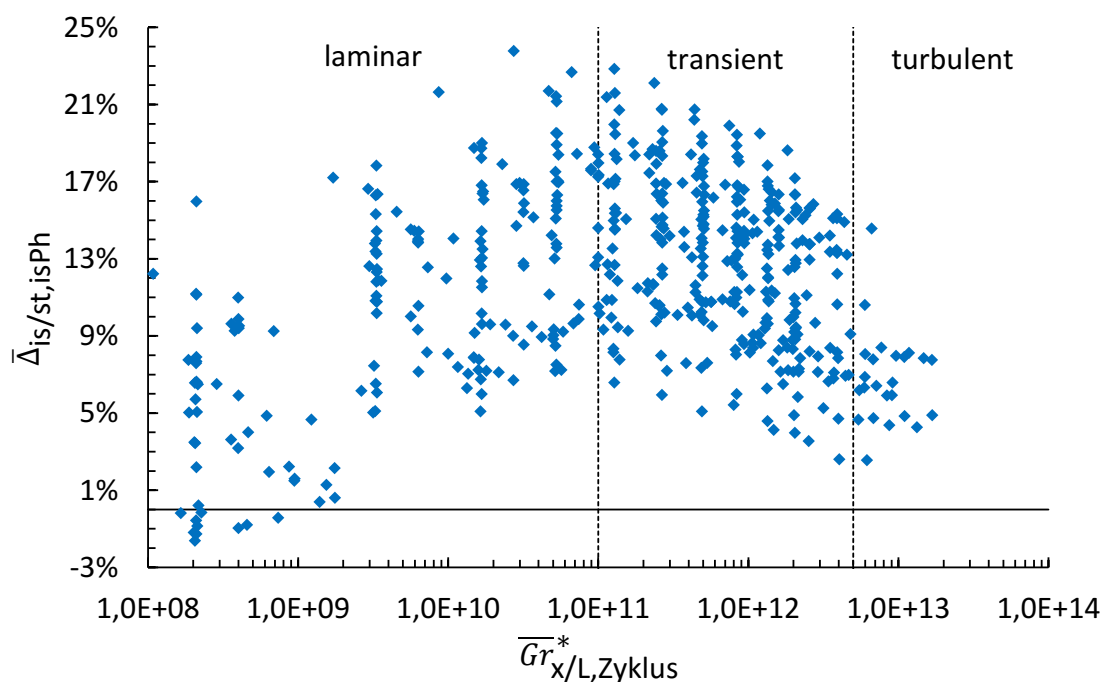

Abbildung 3.24: Zusätzliche konvektive Wärmeübertragung bei zyklischen Variationen (isPh) 
Aufgrund der starken Variation des Anteils der instationären Phase $\Delta t_{\text {isph }}$ an der Gesamt-Zyklusdauer $\Delta t_{\text {Zyklus }}$ werden nachfolgend ausschließlich die mittleren relativen Abweichungen zwischen instationärer und quasi-stationärer Bewertung innerhalb der jeweiligen instationären Phase $\bar{\Delta}_{\mathrm{is} / \mathrm{st}, \text { isph }}$ angegeben. Deren Abhängigkeit von den mittleren Grashof-Zahlen des Gesamt-Zyklus $\overline{G r}_{\mathrm{x} / L, \text { zyklus }}^{*}$ zeigt Abbildung 3.24. Aus der entstandenen Anordnung der Datenpunkte wird zunächst ersichtlich, dass der grundsätzliche Zusammenhang zwischen der Abweichung der instationären Messwerte von der quasi-stationären Betrachtung $\bar{\Delta}_{\text {is/st,isph }}$ und der mittleren Grashof-Zahl $\overline{G r}_{\text {x/L,Zyklus }}^{*}$ unverändert bleibt (vgl. Abbildung 3.20). Eine stärkere Differenzierung der bilanzierten Unterschiede für ähnliche mittlere Grashof-Zahlen lässt jedoch eine deutlichere Ausprägung möglicher Kausalzusammenhänge bei der Analyse sonstiger Einflussgrößen erwarten (siehe Kapitel 5).

\subsubsection{Sprungartige Änderungen mit anschließender Beharrung}

Neben den Versuchsreihen mit zyklisch variierenden Randbedingungen werden auch Änderungen in Form von Sprungantworten mit anschließender Beharrung bei einer definierten (isothermen) Oberflächentemperatur $\vartheta_{\mathrm{W}}$ betrachtet. Hierzu werden 29 Versuchsreihen durchgeführt, aus denen entsprechend der Bilanzierung von Teilabschnitten gemäß Kapitel 1.1.1 insgesamt 290 Betrachtungsfälle resultieren. Die anfangs unbeheizten Sandwich-Heizelemente $\left(\vartheta_{\mathrm{W}}=\vartheta_{\infty}\right)$ werden dabei wiederum einer plötzlichen Leistungszufuhr $\dot{q}_{\text {el }}$ ausgesetzt, die im weiteren Verlauf jedoch mit Hilfe eines reaktionsschnellen PI-Reglers (Verstärkungsfaktor 2,5; Integrationszeit $15 \mathrm{~s}$ ) angepasst wird, um einen vorgegebenen Oberflächen-Übertemperatur-Sollwert $\Delta \vartheta_{\text {Sprung }}$ der Heizelemente zu erreichen und beizubehalten.

In Analogie zu Kapitel 3.4.1 wird zur Beschreibung der WärmestromRandbedingungen der um die Strahlungswärmeabgabe $\dot{q}_{\mathrm{r}}$ bereinigte Wert der elektrischen Leistungszufuhr $\dot{q}_{\text {Impuls }}$ verwendet. Aufgrund der fortlaufenden Anpassung des Wärmestrom-Impulses $\dot{q}_{\text {Impuls }}$ nach Erreichen des OberflächenÜbertemperatur-Sollwerts $\Delta \vartheta_{\text {Sprung }}$ wird im Folgenden dessen Maximalwert $\dot{q}_{\text {max }}$ entsprechend Gleichung 36 zur Charakterisierung der Randbedingungen angegeben. 


$$
\dot{q}_{\max }=\max \left(\dot{q}_{\text {Impuls }}\right)
$$

Im Rahmen der 29 Versuchsreihen mit einer sprungartigen Änderung der Randbedingungen wird die Wärmestromzufuhr auf verschiedene Höchstwerte zwischen $74 \mathrm{~W} / \mathrm{m}^{2} \leq \dot{q}_{\max } \leq 1.430 \mathrm{~W} / \mathrm{m}^{2}$ begrenzt. Die Sollwerte der Oberflächen-Übertemperatur $\Delta \vartheta_{\text {Sprung }}$ werden ferner mit einer Schrittweite von $15 \mathrm{~K}$ zwischen $10 \mathrm{~K} \leq \Delta \vartheta_{\text {Sprung }} \leq 70 \mathrm{~K}$ variiert.

Abbildung 3.25 zeigt dazu beispielhaft den Verlauf der bereinigten Leistungszufuhr $\dot{q}_{\text {Impuls }}$ sowie der Oberflächentemperatur $\vartheta_{\mathrm{W}}$ bei einer maximalen Wärmestromdichte von $\dot{q}_{\max }=1.429 \mathrm{~W} / \mathrm{m}^{2}$ und einem Beharrungs-Sollwert von $\vartheta_{\mathrm{W}}=60^{\circ} \mathrm{C}$.

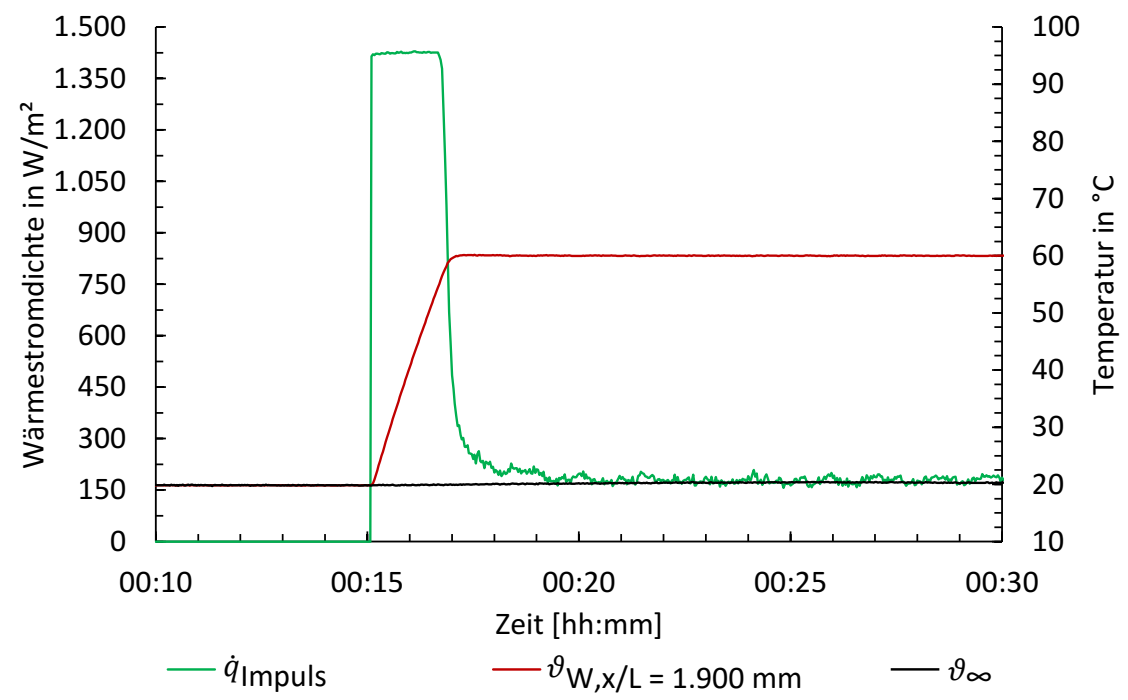

Abbildung 3.25: Temperatur- und Leistungsverlauf bei sprungartigen Änderungen (mit $\dot{q}_{\max }=1.429 \mathrm{~W} / \mathrm{m}^{2}$ und $\Delta \vartheta_{\text {Sprung }}=40 \mathrm{~K}$ )

Die Dauer des Aufheizvorgangs beträgt in der dargestellten Versuchsreihe $\Delta t_{\text {Sprung }}=120 \mathrm{~s}$. Dabei treten mittlere Grashof-Zahlen im Bereich von 5,6 $10^{6}<$ $G r_{\mathrm{x} / \mathrm{L}, \mathrm{m}}<2,7 \cdot 10^{10}$ auf.

In Abbildung 3.26 ist der zugehörige Vergleich zwischen der quasi-stationären Betrachtungsweise $\overline{\dot{q}}_{\mathrm{c}, \mathrm{st}, 90 \mathrm{~s}}$ und den instationären Momentanwerten der konvektiven Wärmeübertragung $\overline{\dot{q}}_{c, i s, 90 \mathrm{~s}}$ dargestellt (vgl. Kapitel 3.4.1). 


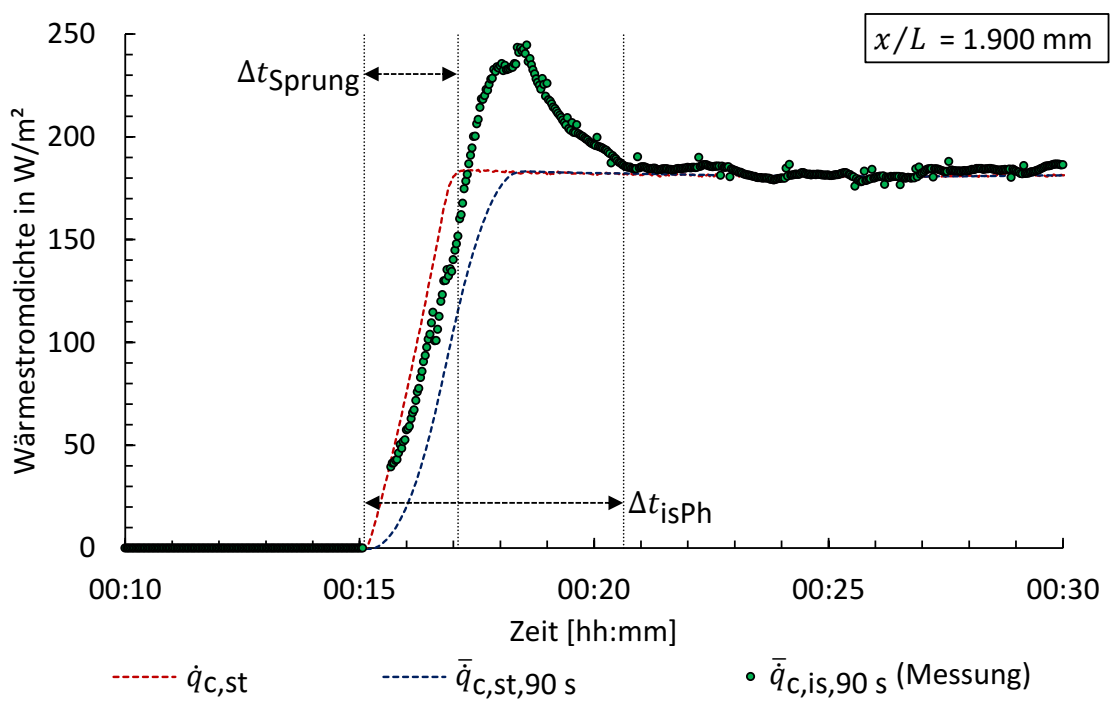

Abbildung 3.26: Konvektive Wärmeübertragung bei sprungartigen Änderungen (mit $\dot{q}_{\text {max }}=1.429 \mathrm{~W} / \mathrm{m}^{2}$ und $\Delta \vartheta_{\text {Sprung }}=40 \mathrm{~K}$ )

In Analogie zu den zyklischen Variationen der Randbedingungen ist auch bei einer sprungartigen Änderung mit anschließender Beharrung eine ausgeprägte Überschreitung der quasi-stationären Vergleichswerte $\overline{\dot{q}}_{c, s t, 90 \text { s }}$ durch die im instationären Fall gemessenen Momentanwerte $\overline{\dot{q}}_{\mathrm{c}, \mathrm{is}, 90 \mathrm{~s}}$ festzustellen. Das Maximum dieser Abweichung tritt ebenfalls zum Zeitpunkt einer rapiden Reduzierung der zugeführten Wärmestromdichte auf und beläuft sich für das dargestellte Strömungsteilgebiet $(x / L=1.900 \mathrm{~mm})$ auf ca. $\Delta_{\mathrm{is} / \mathrm{st}, \max }=36,2 \%$. Die darauffolgende Angleichung der gemessenen Momentanwerte $\overline{\dot{q}}_{\mathrm{c}, \mathrm{is}, 90 \mathrm{~s}}$ an das Niveau der quasi-stationären Betrachtungsweise $\overline{\dot{q}}_{\mathrm{c}, \mathrm{st}, 90 \mathrm{~s}}$ erfolgt bei der hier vorliegenden fortdauernden Leistungszufuhr des Beharrungsniveaus jedoch langsamer, als es bei einer vollständigen Abschaltung der Heizleistung zu beobachten ist (vgl. Kapitel 3.4.1).

Die Dauer der instationären Phase $\Delta t_{\text {isph }}$ erstreckt sich in der dargestellten Versuchsreihe auf $332 \mathrm{~s}$ und entspricht somit in etwa dem 2,8-fachen des Aufheizvorgangs $\Delta t_{\text {sprung. }}$ Im Mittel aller 29 Versuchsreihen zu sprungartigen Variationen der Randbedingungen mit anschließender Beharrung dauert die instationä- 
re Phase $\Delta t_{\text {isph }}$ knapp 2,7-mal länger an, als der eigentliche Aufheizvorgang $\Delta t_{\text {sprung. }}$.

Abbildung 3.27 zeigt eine Übersicht der mittleren Abweichungen zwischen der im instationären Fall erfassten konvektiven Wärmeübertragung und der quasistationären Vergleichsgrundlage innerhalb der jeweiligen instationären Phase $\bar{\Delta}_{\text {is/st,isph }}$ gemäß Gleichung 33 in Abhängigkeit der arithmetischen Mittelwerte der zugehörigen Grashof-Zahlen $G r_{\mathrm{x} / \mathrm{L}, \mathrm{m}}$ für alle 29 Versuchsreihen.

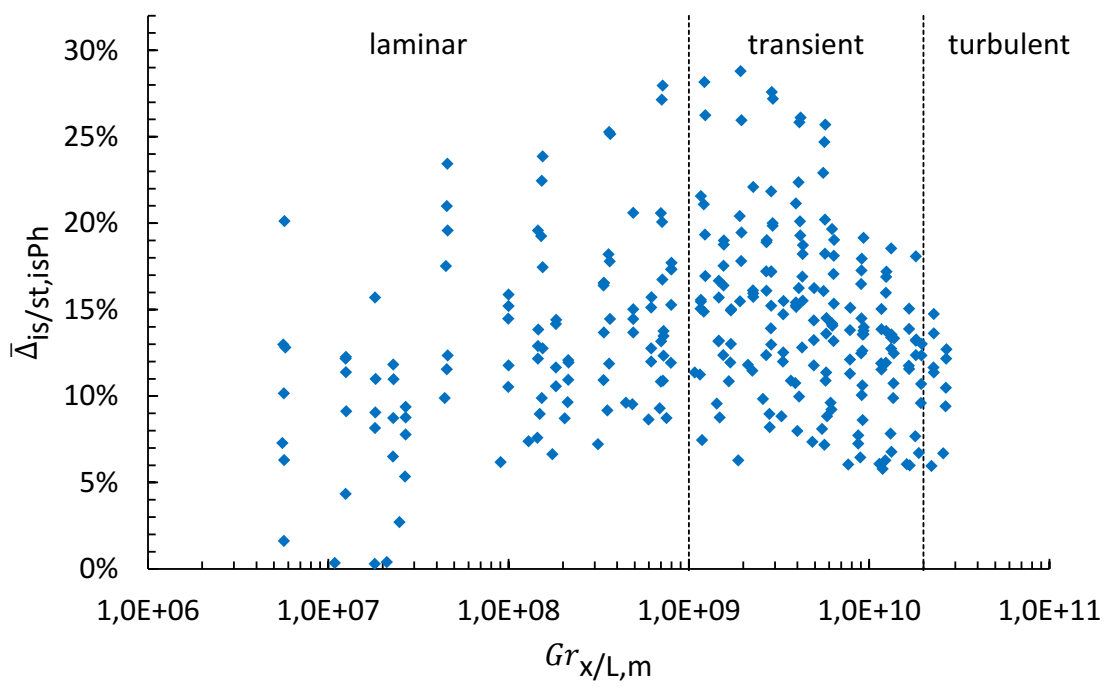

Abbildung 3.27: Zusätzliche konvektive Wärmeübertragung bei sprungartigen Änderungen (isPh)

Die Anordnung der Datenpunkte stellt sich in prinzipieller Ähnlichkeit zu den Versuchsreihen mit zyklisch variierenden Randbedingungen dar. Die Höchstwerte der mittleren Unterschiede zwischen instationären Messwerten und der quasi-stationären Bilanzierung $\bar{\Delta}_{\text {is/st,isph }}$ treten für die sprungartigen Änderungen mit Beharrung jedoch leicht oberhalb der kritischen Grashof-Zahl von $G r_{\mathrm{x} / L, \mathrm{~m}} \approx 10^{9}$, also im Transitionsbereich, auf. Diese Verschiebung wird primär auf die Art und Weise der Versuchsdurchführung zurückgeführt. So werden bei den hier betrachteten Versuchsreihen nach Abschluss des eigentlichen Aufheizvorgangs für die verbleibende Dauer der instationären Phase die Beharrungsniveaus bilanziert, während bei den zyklischen Variationen ein Teil des 
Auskühlvorgangs innerhalb der instationären Phase vollzogen wird. Für mittlere Grashof-Zahlen $G r_{\mathrm{x} / \mathrm{L}, \mathrm{m}}$ außerhalb des Transitionsbereichs fällt die Steigerung der konvektiven Wärmeübertragung durch die instationäre Betriebsweise

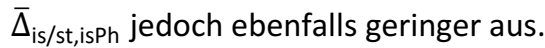

Da Auskühlvorgänge für diese Versuchsreihen ohne Relevanz sind, treten ferner keine bilanziellen Unterschreitungen der quasi-stationären Vergleichswerte $\overline{\dot{q}}_{\mathrm{c}, \mathrm{st}, 90 \mathrm{~s}}$ durch gemessene instationäre Momentanwerte $\overline{\dot{q}}_{\mathrm{c}, \mathrm{is}, 90 \mathrm{~s}}$ am untersten und obersten Heizelement auf (vgl. Kapitel 3.4.1).

\subsection{Schlussfolgerungen zur instationären Wärme- übertragung}

Die im instationären Fall gemessene Intensität der konvektiven Wärmeübertragung nach einer plötzlichen Erhöhung der Wärmestromzufuhr zeigt signifikante Abweichungen zu den Erwartungswerten eines quasi-stationären Verlaufs. Die dabei beobachtete direkte Kopplung dieser Abweichungen an die jeweilige mittlere Grashof-Zahl wird auf die zu Beginn des Transitionsbereichs besonders starke Anfälligkeit der Auftriebsströmung für Stabilitätsstörungen und das dortige lokale Minimum der stationären Wärmeübertragungsintensität (vgl. Kapitel 3.2.3 und 3.2.4) zurückgeführt. Das Auftreten der maximalen Momentanwerte der Abweichungen zum Zeitpunkt einer starken oder vollständigen Reduzierung der Wärmestromzufuhr und die anschließende asymptotische Angleichung an einen quasi-stationären Verlauf implizieren zudem eine Kausalität zwischen der instationären Wärmeübertragungs-Intensität und der jeweiligen Beschaffenheit eines Wärmestrom-Impulses. 


\section{Phänomenologische Betrachtung}

In Ergänzung zur quantitativen Bewertung von instationären Strömungsphänomenen in Kapitel 3 wird nachfolgend eine phänomenologische Beschreibung des Strömungsgebiets bei gezielten Variationen der Strömungs-Randbedingungen vorgenommen. Dies erfolgt mit Hilfe von Strömungsvisualisierungen (Kapitel 4.1) sowie beispielhaft anhand von Messungen der Geschwindigkeits-Fluktuationen innerhalb der Strömungsgrenzschicht (Kapitel 4.2).

\subsection{Strömungsvisualisierung}

Zur Visualisierung der Strömungsverhältnisse des in Kapitel 3.1 beschriebenen Versuchsaufbaus wird an der unteren Kante der beheizten vertikalen Platte ein Propylenglykol-Dampfgemisch (Theater-Nebel) in die Auftriebsströmung eingebracht.

\subsubsection{Versuchsaufbau zur Strömungsvisualisierung}

Die Einbringung des Dampf-Gemisches erfolgt mit Hilfe eines handelsüblichen Schlitzdurchlasses, wie er in der Raumlufttechnik verwendet wird (siehe Abbildung 4.1).

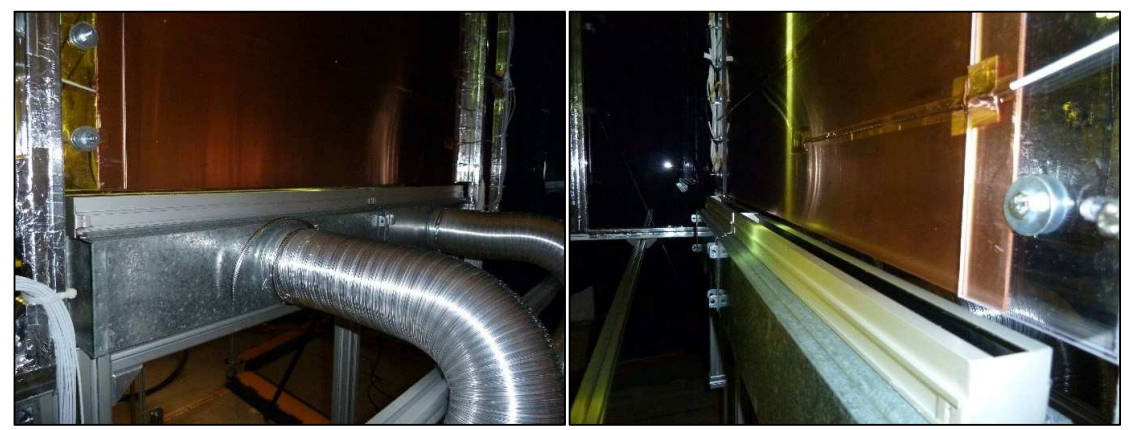

Abbildung 4.1: Dampf-Einbringung zur Strömungsvisualisierung

Zur Reduktion des Freistrahl-Impulses des verwendeten Dampferzeugers (Typ hazebase base*prime) und zur Temperaturanpassung wird das Dampfgemisch vor der Einbringung in die Auftriebsströmung durch ein insgesamt ca. $25 \mathrm{~m}$ langes Aluminium-Wickelfalzrohr (DN 160) geleitet, das außenseitig an die Umge- 
bungsluft der Versuchshalle grenzt. Die Rohrlänge wird dabei in einem iterativen Prozess solange variiert, bis sich eine nahezu dichteneutrale und möglichst impulsarme Austritts-Situation an der Plattenunterkante einstellt. Für eine Erhöhung des optischen Kontrastes zwischen der dampfhaltigen Grenzschichtströmung und der Umgebungsluft werden die Innenwände des Versuchsaufbaus zudem schwarz ausgekleidet und eine Linienprojektion (Lichtschnitt, Typ Philips PCV740) orthogonal zur beheizten Plattenoberfläche implementiert. Darüber hinaus wird aus konstruktiven Gründen die untere Ebene der vertikalen Strömungs-Gleichrichter (vgl. Kapitel 3.1.1) für die Dauer der Strömungsvisualisierungen entfernt.

\subsubsection{Strömungsstrukturen}

Zunächst wird die Auftriebsströmung an der vertikalen beheizten Platte im strömungsmechanisch vollständig ausgebildeten (statistisch stationären) Fall betrachtet. Wie aus Abbildung 4.2 hervor geht, entsprechen die auftretenden Strömungsstrukturen dabei der in Kapitel 1.2 erläuterten Phänomenologie. So folgen auf die geordneten Stromlinien im unteren Plattenbereich zunehmend mehrdimensionale Strukturen, die schließlich in vollturbulente Elemente zerfallen.

Bei einer Veränderung der stationären Strömungs-Randbedingungen in Analogie zu Kapitel 3.4 sind hingegen temporär deutliche Veränderungen der Grenzschichtdicke und der lokal auftretenden Strömungsstrukturen festzustellen. Abbildung 4.3 und Abbildung 4.4 zeigen dazu die Entwicklung einer zunächst stationären Auftriebsströmung (Abbildung 4.3, links) bei einer Übertemperatur der isothermen Plattenoberfläche zur Umgebung von ca. $\Delta \vartheta_{\mathrm{W}-\infty}=7,5 \mathrm{~K}$, der für eine Dauer von $\Delta t_{\text {Impuls }}=30 \mathrm{~s}$ eine sprungartig erhöhte Wärmestromdichte von etwa $\dot{q}_{\text {Impuls }}=1.430 \mathrm{~W} / \mathrm{m}^{2}$ aufgeprägt wird.

Der plötzliche Energieeintrag in den Heizelementen wird zunächst durch Wärmeleitung an die wandnächsten Fluidteilchen abgeführt, wodurch sich deren Temperatur erhöht und sich deren Dichte entsprechend reduziert. Die gesteigerte potentielle Energie der betreffenden Fluidteilchen wird daraufhin durch Beschleunigung (Abbildung 4.3, Mitte) in kinetische Energie umgewandelt. Die beschleunigten Teilchen stehen dabei im Impulsaustausch mit ruhendem oder langsamerem Umgebungsfluid. 


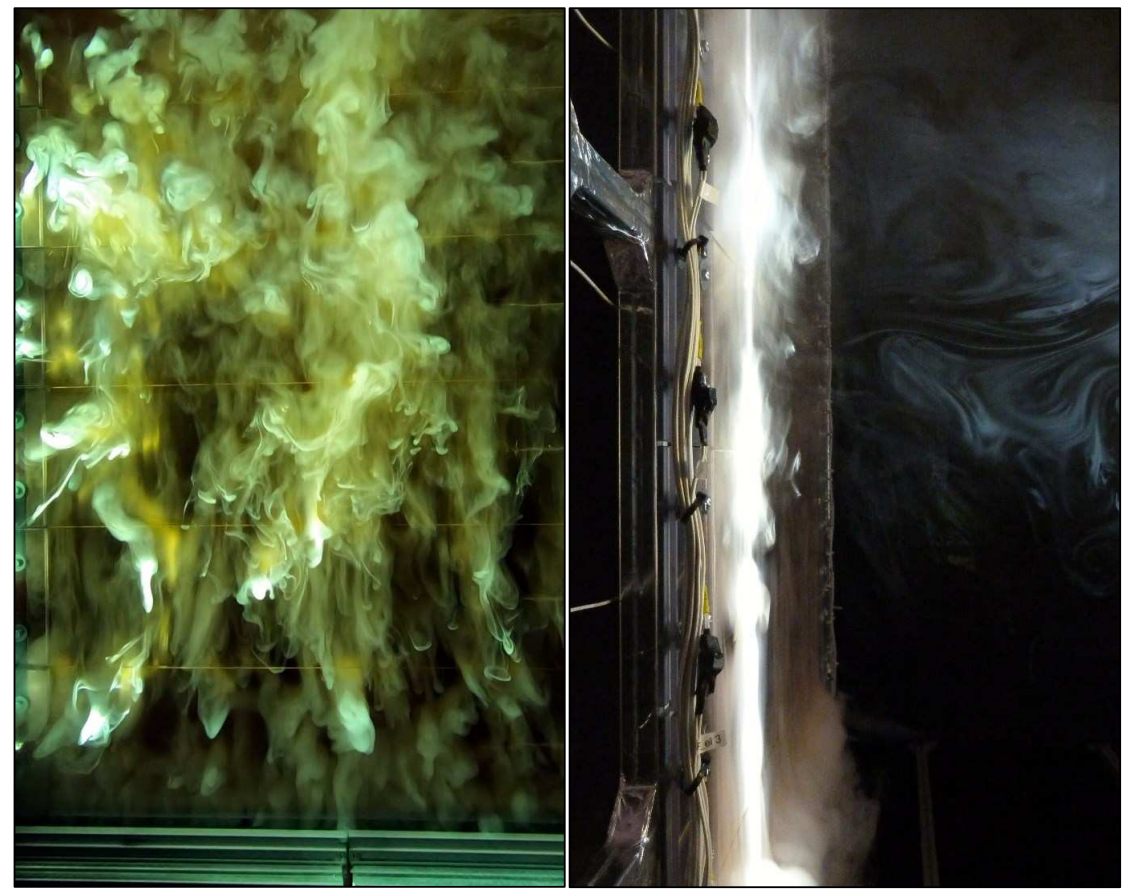

Abbildung 4.2: Visualisierte Grenzschichtströmung im stationären Zustand (links: Plattenansicht; rechts: Strömungsprofil)

Durch Viskositätskräfte und lokale Druckunterschiede aufgrund der verschiedenen Strömungsgeschwindigkeiten kommt es zu Scherbewegungen (Abbildung 4.3, rechts), die sich im weiteren Verlauf zu rotierenden Strukturen fortpflanzen (Abbildung 4.4, links). Besonders häufig sind dabei die für Scherschichten zwischen unterschiedlich schnellen Fluiden typischen KelvinHelmholtz-Wirbel zu beobachten (Abbildung 4.4, Mitte). Die verstärkte Durchmischung der Strömungsgrenzschicht bewirkt eine erhöhte Induktion von kaltem Umgebungsfluid in die Auftriebsströmung, was als ursächlich für die in Kapitel 3.4 festgestellte temporäre Intensivierung der konvektiven Wärmeübertragung interpretiert wird. Mit zunehmendem Abbau des kurzfristig eingebrachten Energieüberschusses durch konvektive Transportvorgänge wird das stationäre Verhältnis zwischen potentieller und kinetischer Energie schließlich wiederhergestellt und die zusätzlichen, großskaligen Rotationsstrukturen bilden sich zurück (Abbildung 4.4, rechts). 


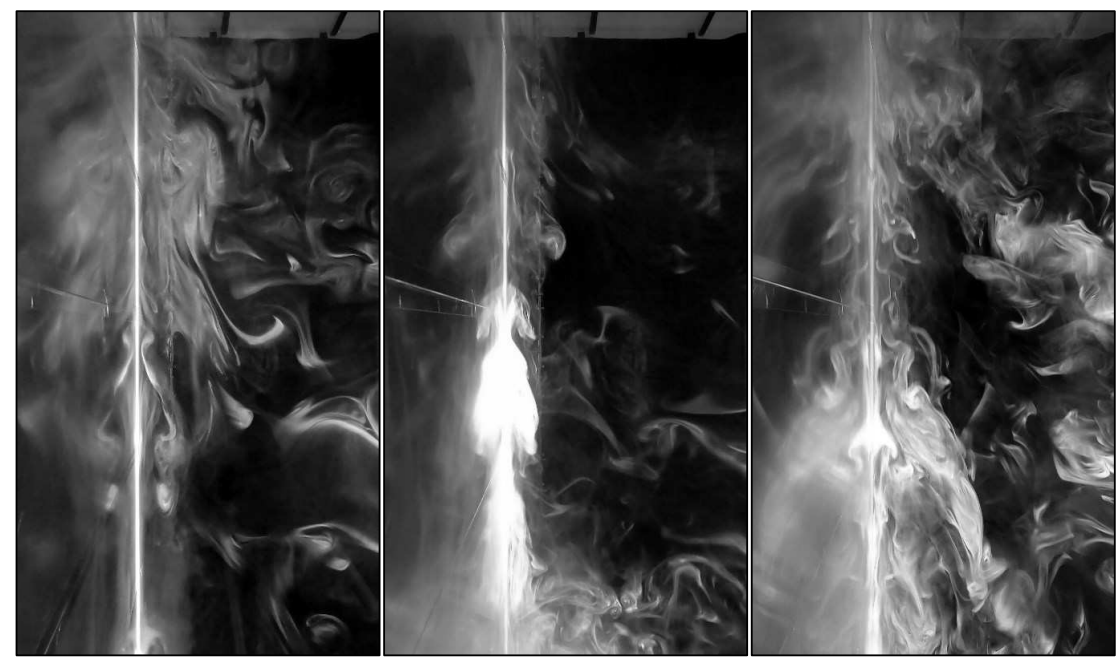

Abbildung 4.3: Strömungsstrukturen bei Wärmestromimpuls

(mit $\dot{q}_{\text {Impuls }}=1.430 \mathrm{~W} / \mathrm{m}^{2}$ und $\Delta t_{\text {Impuls }}=30 \mathrm{~s}$ )

(links: $27,5{ }^{\circ} \mathrm{C}$ stationär; Mitte: Impulsbeginn +13 s; rechts: Impulsbeginn +25 s)

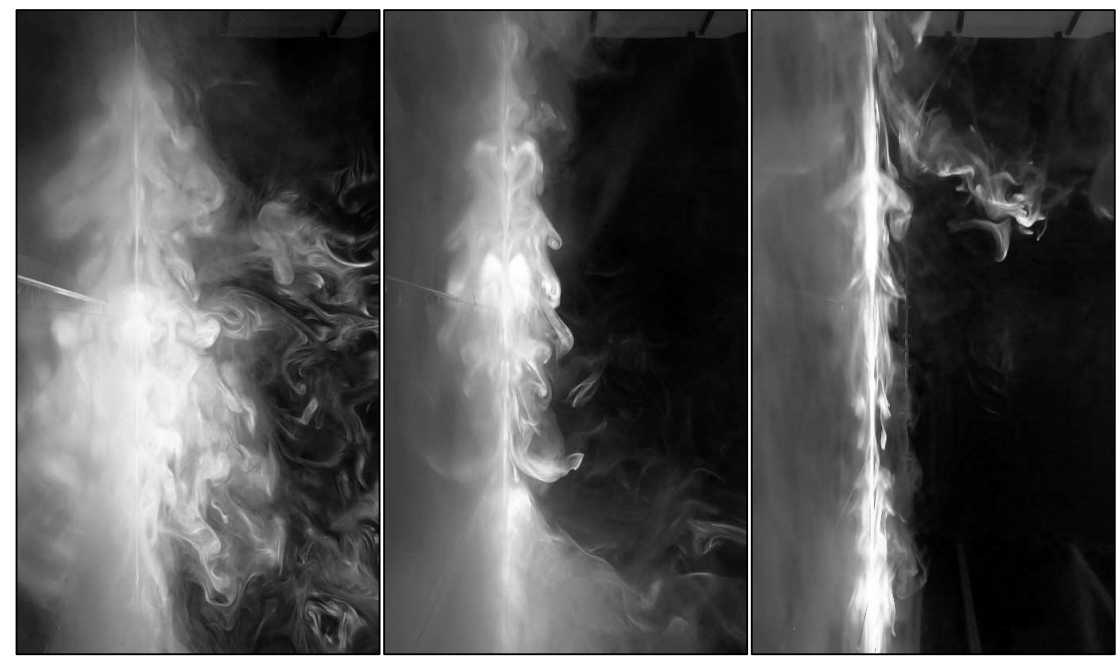

Abbildung 4.4: Strömungsstrukturen nach Wärmestromimpuls (mit $\dot{q}_{\text {Impuls }}=1.430 \mathrm{~W} / \mathrm{m}^{2}$ und $\Delta t_{\text {Impuls }}=30 \mathrm{~s}$ )

(links: Impulsende +2 s; Mitte: Impulsende +34 s; rechts: Impulsende +164 s) 


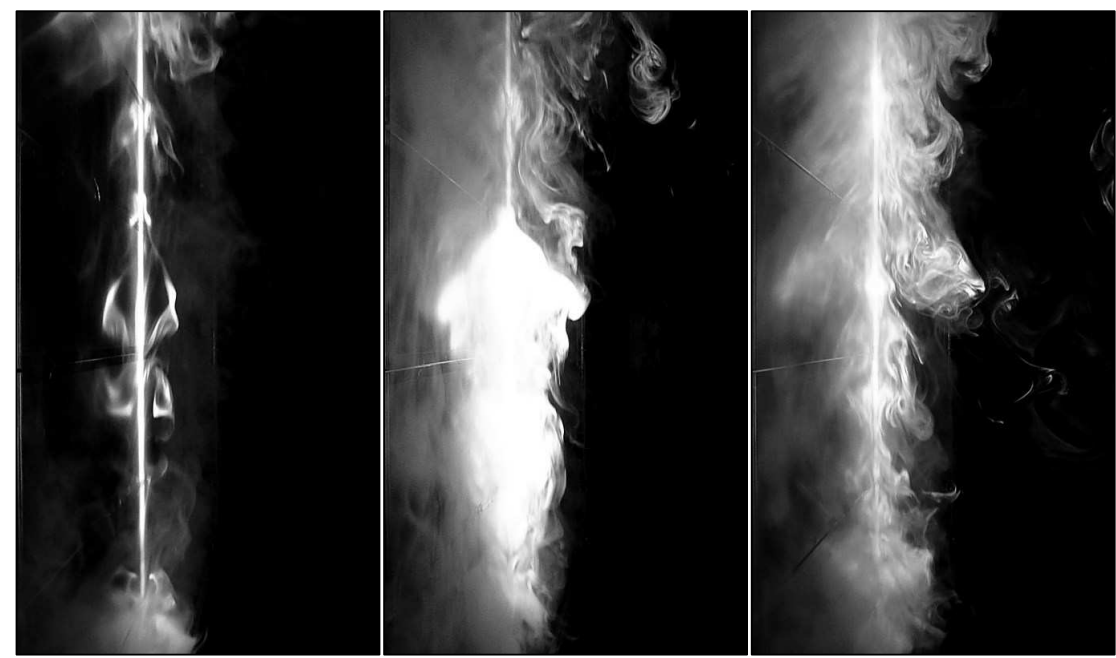

Abbildung 4.5: Strömungsstrukturen bei Temperatursprung (mit $\dot{q}_{\max }=711 \mathrm{~W} / \mathrm{m}^{2}$ und $\Delta \vartheta_{\text {Sprung }}=40 \mathrm{~K}$ ) (links: $30^{\circ} \mathrm{C}$ stationär; Mitte: Impulsbeginn +9 s; rechts: Impulsbeginn +45 s)

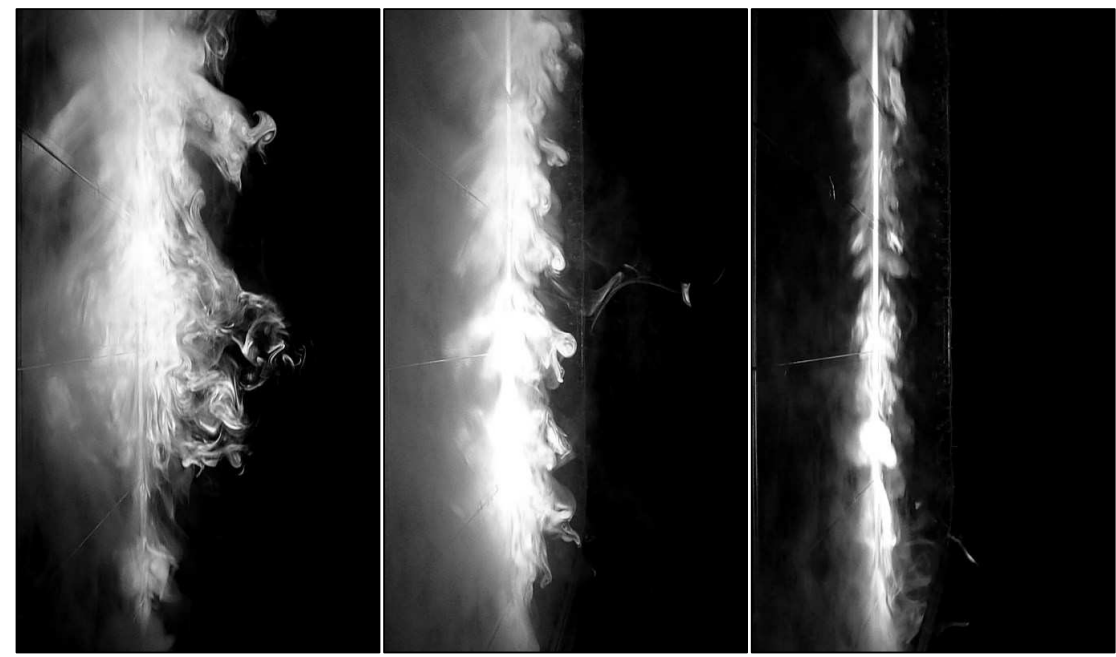

Abbildung 4.6: Strömungsstrukturen nach Temperatursprung (mit $\dot{q}_{\text {max }}=711 \mathrm{~W} / \mathrm{m}^{2}$ und $\Delta \vartheta_{\text {Sprung }}=40 \mathrm{~K}$ )

(links: Impulsbeginn +119 s; Mitte: Impulsbeginn $+168 \mathrm{~s}$; rechts: $70{ }^{\circ} \mathrm{C}$ stationär)

In Analogie zu Kapitel 3.4 wird neben zyklischen Variationen der Randbedingungen auch die Entwicklung der Strömungsstrukturen bei sprungartigen Rand- 
bedingungs-Änderungen mit anschließender Beharrung betrachtet. Abbildung 4.5 und Abbildung 4.6 zeigen dazu vergleichbare Entwicklungsstadien für eine Versuchsreihe mit einer anfangs isothermen Übertemperatur der beheizten Platte von $\Delta \vartheta_{\mathrm{W}-\infty}=10 \mathrm{~K}$ (Abbildung 4.5, links), die dann sprungartig mit einer maximalen Wärmestromdichte von ca. $\dot{q}_{\max }=711 \mathrm{~W} / \mathrm{m}^{2}$ um ca. $\Delta \vartheta_{\text {Sprung }}=40 \mathrm{~K}$ erwärmt wird. Grundsätzlich sind dabei die gleichen temporären Durchmischungseffekte der Strömungsgrenzschicht zu beobachten, wie im Falle der zyklischen Variationen (Abbildung 4.5, Mitte bis Abbildung 4.6, Mitte). In Übereinstimmung mit den gemessenen Momentanwerten der konvektiven Wärmeübertragung der Abbildung 3.26 in Kapitel 3.4.2 ist für sprungartige Änderungen der Strömungs-Randbedingungen mit anschließender Beharrung aufgrund der fortdauernden Wärmestrom-Zufuhr jedoch eine länger andauernde instationäre Phase zu beobachten, bevor die Auftriebsströmung wieder ausschließlich quasi-stationäre Strukturen aufweist (Abbildung 4.6, rechts).

Die beschriebene zeitliche Entwicklung der Strömungsphänomenologie kann für die betrachteten Randbedingungs-Variationen zuverlässig reproduziert werden. Für unterschiedliche Impuls-Intensitäten, Zyklusdauern und Beharrungsniveaus zeigen sich insbesondere Variationen in der Aus- und Rückbildungsdauer sowie in der räumlichen Ausdehnung der temporären Rotationsstrukturen.

Darüber hinaus zeigen Visualisierungen von [Joshi und Gebhart 1987] sowie [Joshi und Gebhart 1988] (siehe auch Kapitel 2.1) für eine Auftriebsströmung in Wasser nach einer sprungartigen Erhöhung oder Reduzierung der zugeführten Wärmestromdichte eine ähnliche strukturelle und zeitliche Entwicklung.

\subsection{Strömungsgeschwindigkeiten}

Eine messtechnische Erfassung des zeitlichen Verlaufs von Geschwindigkeiten innerhalb der Auftriebsströmung an der vertikalen Platte erfolgt mit Hilfe von omnidirektionalen Hitzkugel-Anemometern (Typ Dantec 54T21S). Aufgrund einer möglichen Beeinflussung der auftretenden Strömungsstrukturen durch die invasive Mess-Methodik liegt der Fokus der Betrachtungen jedoch auf einer exemplarischen Darstellung von Fluktuationen. Absolute Geschwindigkeiten sind im stationären Fall bereits aus zahlreichen Betrachtungen bekannt (vgl. 
z.B. [Miyamoto und Okayama 1982], [Tsuji und Nagano 1989] sowie zusammenfassend [Oertel jr. 2017]). Für eine darüber hinaus gehende quantitative Bestimmung des zeitlichen Verlaufs von Absolut-Geschwindigkeiten bei einer gezielten Variation der Strömungs-Randbedingungen erscheint neben einer nicht-invasiven Mess-Methodik zudem eine statistische Überprüfung der zeitlichen und örtlichen Reproduzierbarkeit sinnvoll.

\subsubsection{Versuchsaufbau zur Messung von Strömungsgeschwindigkeiten}

Die Hitzkugel-Anemometer werden im experimentellen Aufbau gemäß Kapitel 3.1 mit unterschiedlichen Abständen zur beheizten vertikalen Platte ( $y=5 \mathrm{~mm}$, $y=10 \mathrm{~mm}, y=25 \mathrm{~mm}, y=50 \mathrm{~mm}$ und $y=75 \mathrm{~mm}$ ) sowie bei verschiedenen Strömungsweglängen $(x / L=700 \mathrm{~mm}, x / L=1.300 \mathrm{~mm}$ und $x / L=1.900 \mathrm{~mm})$ positioniert. Die Anordnung in $z$-Richtung erfolgt in Plattenmitte. Abbildung 4.7 veranschaulicht die entsprechende Implementierung im Versuchsaufbau.

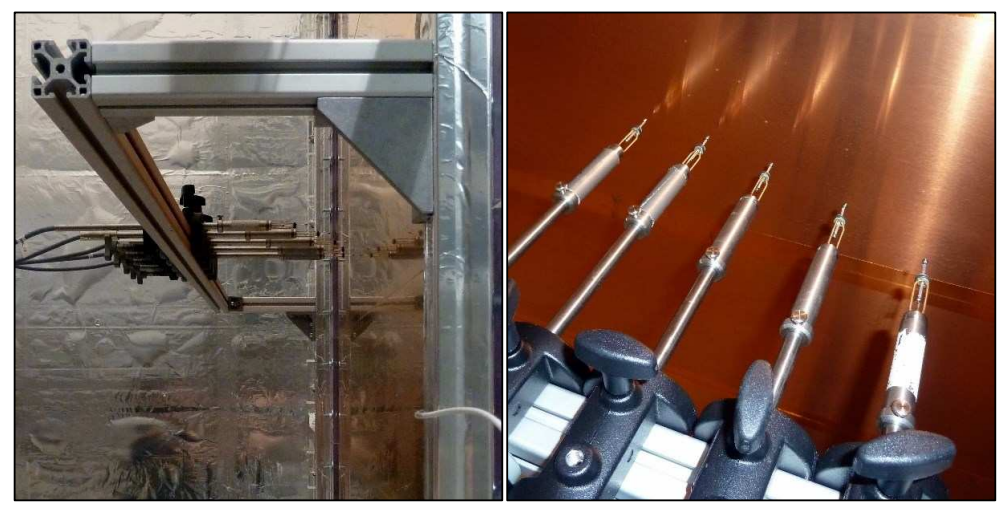

Abbildung 4.7: Anordnung der Hitzkugel-Anemometer

Aufgrund der Richtungsunabhängigkeit der verwendeten Hitzkugel-Anemometer beinhalten die dargestellten Geschwindigkeitsfluktuationen Anteile aller drei Richtungs-Komponenten ( $x, y$ und $z$ ). Aus Gründen der Lesbarkeit werden die kombinierten Schwankungsgrößen nachfolgend hingegen als Geschwindigkeitskomponente $u$ der dominierenden $x$-Richtung bezeichnet. Ein quantitativer Vergleich der gemessenen Geschwindigkeitsfluktuationen mit den in Kapitel 2.1 genannten Untersuchungen ist dementsprechend jedoch nicht möglich. 


\subsubsection{Geschwindigkeits-Fluktuationen}

Abbildung 4.8 zeigt beispielhaft den Verlauf der Oberflächentemperatur der beheizten Platte $\vartheta_{\mathrm{W}}$ sowie der dimensionslosen Lokalgeschwindigkeit $u_{\mathrm{x}}^{+}$gemäß Gleichung 12 und des zugehörigen Turbulenzgrades $T u_{\mathrm{x}}$ nach Gleichung 11 an der Strömungsposition $x=1.300 \mathrm{~mm}$ bei einem Plattenabstand von $y=50 \mathrm{~mm}$ für eine zyklische Variation der Wärmestromdichte. Dabei wird für $\Delta t_{\text {Impuls }}=120 \mathrm{~s}$ ein Wärmestromimpuls von ca. $\dot{q}_{\text {Impuls }}=1.430 \mathrm{~W} / \mathrm{m}^{2}$ aufgeprägt, dem eine Auskühlphase von $\Delta t_{\text {Auskühlung }}=1.800 \mathrm{~s}$ folgt.

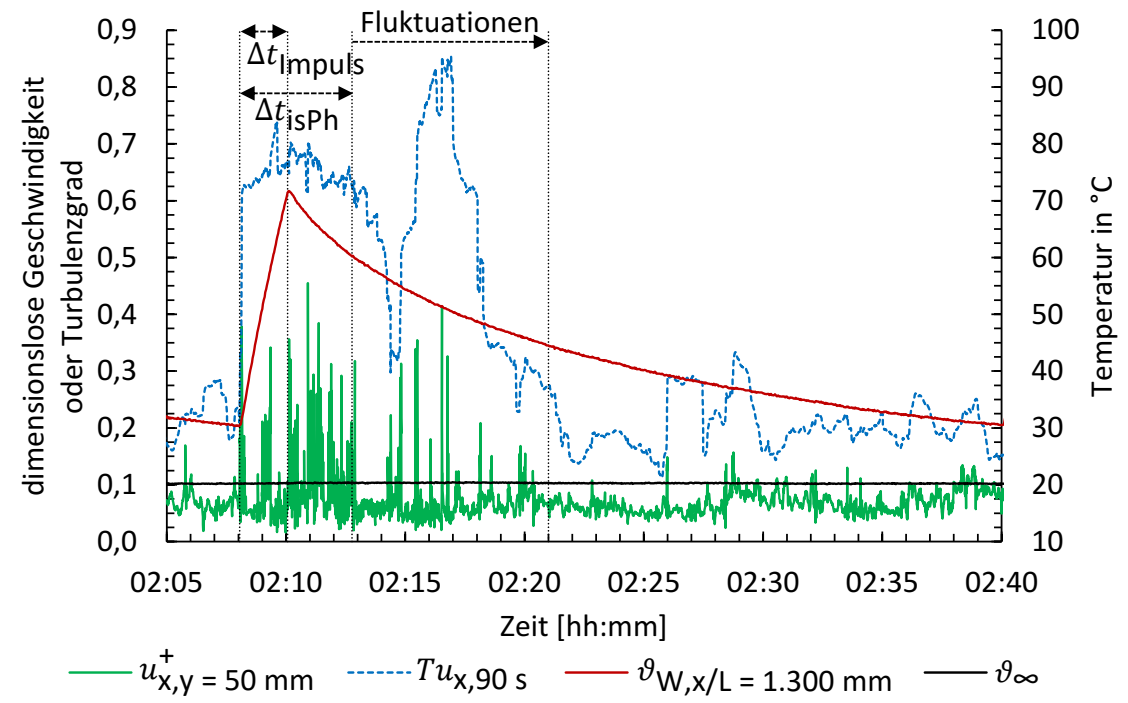

Abbildung 4.8: Zeitlicher Verlauf der Strömungsgrößen bei $x=1.300 \mathrm{~mm}$ (mit $\dot{q}_{\text {Impuls }}=1.430 \mathrm{~W} / \mathrm{m}^{2}, \Delta t_{\text {Impuls }}=120 \mathrm{~s}$ und $\Delta t_{\text {Auskühlung }}=1.800 \mathrm{~s}$ )

Die Differenz zwischen der mittleren Oberflächentemperatur des StrömungsTeilgebietes und der ungestörten Fluidtemperatur $\Delta \vartheta_{\mathrm{W}-\infty}$ variiert in der betrachteten Versuchsreihe von 9,3 K bis $51 \mathrm{~K}$; das zeitliche Mittel liegt bei ca. 23,1 K. Die zugehörigen Grashof-Zahlen variieren dementsprechend in einem Bereich von $4,3 \cdot 10^{11}<G r_{x / L}^{*}<2,9 \cdot 10^{12}$, wobei der zeitliche Mittelwert $\overline{G r}_{\mathrm{x} / L, \text { Zyklus }}^{*}=1,6 \cdot 10^{12}$ beträgt.

Die zu erwartende Dicke der lokalen Strömungsgrenzschicht kann im stationären Fall für eine laminare (siehe z.B. [Oertel jr. 2017]) oder turbulente (vgl. z.B. 
[Cheeswright 1968]) Strömungscharakteristik abgeschätzt werden. Für die minimale Platten-Übertemperatur von $\Delta \vartheta_{\mathrm{W}-\infty}=9,3 \mathrm{~K}$ liegt diese an der dargestellten Stelle des Strömungsgebiets $(x / L=1.300 \mathrm{~mm}$ ) zwischen $\delta=44 \mathrm{~mm}$ (laminar) und $\delta=192 \mathrm{~mm}$ (turbulent). Bei der maximalen Übertemperatur von $\Delta \vartheta_{\mathrm{W}-\infty}=51 \mathrm{~K}$ ist von einer lokalen Grenzschichtdicke zwischen $\delta=31 \mathrm{~mm}$ und $\delta=166 \mathrm{~mm}$ auszugehen. Die betrachtete Messposition $x=1.300 \mathrm{~mm}$ mit einem Plattenabstand von $y=50 \mathrm{~mm}$ liegt für die minimale PlattenÜbertemperatur von $\Delta \vartheta_{\mathrm{W}-\infty}=9,3 \mathrm{~K}$ im stationären Fall folglich eher im äußeren Bereich der dann überwiegend laminaren Strömungsgrenzschicht. Während der (stationär) als überwiegend turbulent einzustufenden Strömung der maximalen Übertemperatur von $\Delta \vartheta_{\mathrm{W}-\infty}=51 \mathrm{~K}$ befindet sich das HitzkugelAnemometer hingegen eher im inneren Grenzschichtbereich.

Wie aus Abbildung 4.8 deutlich ersichtlich wird, schwankt die dimensionslose Geschwindigkeit $u_{x}^{+}$für die betrachtete zyklische Variation der StrömungsRandbedingungen überwiegend in einem Bereich von unter 0,1. Mit Beginn des Aufheiz-Impulses $\Delta t_{\text {Impuls }}$ sowie einige Zeit danach sind hingegen deutlich intensivere Geschwindigkeits-Fluktuationen zu erkennen, deren Momentanwerte das zeitliche Mittel des gesamten Zyklus um ein Vielfaches (im Maximum ca. Faktor 6,6) überschreiten.

Der Zeitraum mit den stärksten Geschwindigkeits-Fluktuationen steht in guter Übereinstimmung mit der Dauer der instationären Phase $\Delta t_{\text {isPh, }}$ die gemäß Gleichung 31 eine Abweichung von mehr als einem Prozent zwischen dem Momentanwert der konvektiven Wärmeübertragung und dem quasistationären Vergleichswert aufweist. Für die gesamte instationäre Phase der betrachteten Versuchsreihe von ca. $\Delta t_{\text {isph }}=282 \mathrm{~s}$ resultiert nach Gleichung 33 eine mittlere Abweichung der instationären konvektiven Wärmeübertragung gegenüber der quasi-stationären Betrachtung von $\bar{\Delta}_{\text {is/st,isph }}=16 \%$.

In Anlehnung an Kapitel 3.3.2 wird die Grenzschichtgeschwindigkeit $u_{\mathrm{x}}^{+}$zur Bestimmung des lokalen Turbulenzgrads $T u_{\mathrm{x}}$ ebenfalls für einen Bilanz-Zeitschritt von $\Delta t=90 \mathrm{~s}$ ausgewertet. In Abbildung 4.8 ist dementsprechend der zeitliche Verlauf des Turbulenzgrades der jeweils letzten $90 \mathrm{~s} T u_{\mathrm{x}, 90 \mathrm{~s}}$ dargestellt. Bezogen auf die Dauer der instationären Phase $\Delta t_{\text {isph }}$ liegt der mittlere Turbulenzgrad bei $\overline{T u} \bar{u}_{\text {,isph }}=68 \%$ und damit deutlich über dem zeitlichen Mittel der restlichen Zyklusdauer von $\overline{T u}_{\mathrm{x}}=36 \%$. Darüber hinaus treten auch nach Abschluss 
der instationären Phase noch vereinzelte, jedoch ähnlich intensive Geschwindigkeits-Fluktuationen auf. In der danach verbleibenden Zyklusdauer (ca. 02:21 bis Zyklusende) reduziert sich der mittlere Turbulenzgrad auf $\overline{T u}_{\mathrm{x}}=28 \%$. Während der Fluktuationen, die nach Abschluss der instationären Phase auftreten, liegen an der betrachteten Strömungsposition $(x / L=1.300 \mathrm{~mm})$ GrashofZahlen von $2,3 \cdot 10^{12}>G r_{\mathrm{x} / \mathrm{L}}^{*}>1,2 \cdot 10^{12}$ vor. Im betreffenden Zeitraum befindet sich die Messstelle also im Kernbereich der laminar-turbulenten Transition. Es liegt daher die Vermutung nahe, dass die beschriebenen nachgelagerten Fluktuationen auf die lokale, quasi-stationäre Strömungstransition zurückzuführen sind.

Abbildung 4.9 zeigt in Ergänzung die zeitliche Entwicklung der lokalen Strömungsgeschwindigkeit $u_{\mathrm{x}}^{+}$und des zugehörigen Turbulenzgrades $T u_{\mathrm{x}, 90 \mathrm{~s}}$ für eine identische Versuchsreihe, jedoch bei einer Messposition von $x=700 \mathrm{~mm}$ und einem Plattenabstand von $y=5 \mathrm{~mm}$. Irreguläre Schwankungen treten dort ausschließlich innerhalb der instationären Phase auf. Eine quasi-stationäre Betrachtungsweise ließe an der dargestellten Strömungsposition $(x / L=700 \mathrm{~mm})$ hingegen selbst für die maximale Temperaturdifferenz von $\Delta \vartheta_{\mathrm{W}-\infty}=51 \mathrm{~K}$ noch eine nahezu vollständig laminare Strömung erwarten $\left(G r_{\mathrm{x} / L \text {, } \max }^{*} \approx 2,5 \cdot 10^{11}\right)$.

Die in Abbildung 4.9 ersichtliche Entwicklung einer zunehmenden Lokalgeschwindigkeit $u_{\mathrm{x}}^{+}$bei abnehmender Temperaturdifferenz zwischen Plattenoberfläche und Umgebungsfluid $\Delta \vartheta_{\mathrm{W}-\infty}$ ist auf die zeitliche Veränderung des Geschwindigkeitsprofils (vgl. Abbildung 1.1) an der Messstelle zurückzuführen. So verschiebt sich die im stationären Fall zu erwartende Position des Geschwindigkeitsmaximums innerhalb der Strömungsgrenzschicht von ca. $y=4,2 \mathrm{~mm}$ bei der maximalen Temperaturdifferenz von $\Delta \vartheta_{\mathrm{W}-\infty}=51 \mathrm{~K}$ auf etwa $y=5,9 \mathrm{~mm}$ bei der minimalen Platten-Übertemperatur von $\Delta \vartheta_{\mathrm{W}-\infty}=9,3 \mathrm{~K}$ (vgl. z.B. [Oertel jr. 2017]). Dementsprechend liegt die Messposition der dargestellten Versuchsreihe $(y=5 \mathrm{~mm}$ ) bei den hohen Oberflächentemperaturen weiter von der Plattenoberfläche entfernt, als der Punkt der maximalen Grenzschichtgeschwindigkeit. Mit abnehmender Temperaturdifferenz nimmt die Dicke der laminaren Strömungsgrenzschicht zu, wodurch sich deren Geschwindigkeitsmaximum zunehmend in Richtung der Messposition (und darüber hinaus) verlagert. In der Folge steigt die Lokalgeschwindigkeit an der Messposition kontinuierlich an. 


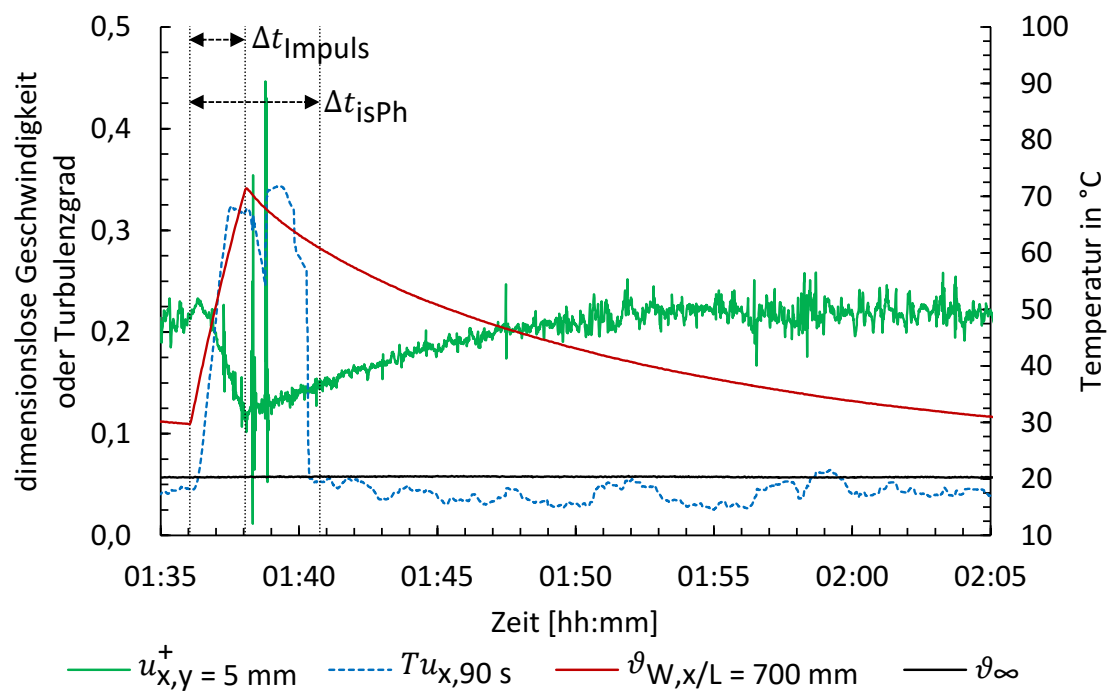

Abbildung 4.9: Zeitlicher Verlauf der Strömungsgrößen bei $x=700 \mathrm{~mm}$ (mit $\dot{q}_{\text {Impuls }}=1.430 \mathrm{~W} / \mathrm{m}^{2}, \Delta t_{\text {lmpuls }}=120 \mathrm{~s}$ und $\Delta t_{\text {Auskühlung }}=1.800 \mathrm{~s}$ )

Qualitativ sowie bezüglich der zeitlichen Entwicklung zeigen die erfassten Geschwindigkeits-Fluktuationen darüber hinaus eine gute Kongruenz zu bereits bekannten Messungen (vgl. [Joshi und Gebhart 1987], [Joshi und Gebhart 1988] sowie [Zhao et al. 2015] in Abbildung 2.1).

\subsection{Schlussfolgerungen zu instationären Strömungs- strukturen}

Sowohl die Visualisierung der verstärkten Grenzschicht-Durchmischung, als auch die gute zeitliche Übereinstimmung der gemessenen GeschwindigkeitsFluktuationen mit der instationären Phase der konvektiven Wärmeübertragung deuten auf eine signifikante Beeinflussung des Strömungsfeldes durch eine sprungartige Erhöhung der Wärmestromzufuhr hin. In Abhängigkeit von der Art und Weise der Wärmestromänderung sind dabei temporär instationäre Phänomene zu beobachten, die deutlich von einer quasi-stationären Strömungsentwicklung abweichen. 


\section{Analytische Prognose der Wärmeübertragung durch instationäre freie Konvektion}

Für die in Kapitel 4 beobachtete zeitliche Entwicklung von Auftriebsströmungen nach einer plötzlichen Veränderung der Strömungs-Randbedingungen wird nachfolgend zunächst eine physikalische Interpretation vorgestellt (Kapitel 5.1), auf deren Grundlage ein Modellansatz zur Prognose der Wärmeübertragung durch instationäre freie Konvektion an der vertikalen Platte erarbeitet wird. Anhand der in Kapitel 3 für verschiedenartige Randbedingungs-Variationen experimentell erfassten Abweichungen zwischen einer instationären Betriebsweise und einer quasi-stationären Betrachtung erfolgt im Anschluss die mathematische Gestaltung einer analytischen Berechnungsmethodik. Die relevanten Modellparameter werden schließlich mit Hilfe von mehrfach reproduzierten, systematischen Validierungsmessungen bestimmt (Kapitel 5.2 und 5.3).

\subsection{Physikalische Modellvorstellung}

Die in Kapitel 2.2 dargestellten Transport-Gleichungen für Masse, Impuls und Energie setzen für die Beschreibung von freien Konvektionsströmungen sowohl im stationären, als auch im instationären Fall eine unmittelbare Kopplung zwischen Geschwindigkeits- und Temperaturfeld voraus. So wird ein Teil der durch Wärmeleitung von der beheizten Oberfläche abgeführten Energie im Fluid von potentieller in kinetische Energie umgewandelt (vgl. Kapitel 4.1.2). Die verbleibende Energiemenge dissipiert aufgrund von Zähigkeits- und Trägheitskräften.

\subsubsection{Potentielle und kinetische Energie der freien Konvektion}

Für den Fall einer vollständig ausgebildeten, statistisch stationären Strömung ist von einem konstanten Verhältnis zwischen potentieller und kinetischer Energie der Fluidteilchen auszugehen (vgl. z.B. [Wagner 2011]). Die phänomenologischen Beobachtungen aus Kapitel 4.1.2 begründen demgegenüber die Annahme, dass das Verhältnis von potentieller zu kinetischer Energie im Falle einer plötzlichen Änderung der Strömungs-Randbedingungen temporär variiert. So muss die zusätzlich in das Fluid eingebrachte potentielle Energie zunächst durch Beschleunigung in kinetische Energie umgewandelt werden, bevor sich 
das stationäre Verhältnis (wieder) einstellt. Vereinfacht ausgedrückt wird dies als Abbau von überschüssiger potentieller Energie durch das vorübergehende Aufkommen von instationären Strömungsstrukturen interpretiert.

Zur Beschreibung der Wärmeübertragung durch eine solche instationäre freie Konvektionsströmung mit Hilfe einer analytischen Berechnungsmethodik liegt es daher nahe, neben der in Kapitel 3.4 als Regressionsparameter identifizierten mittleren Grashof-Zahl auch die zeitliche Variabilität des Verhältnisses von potentieller zu kinetischer Energie zu berücksichtigen.

Die spezifische potentielle Energie im Gravitationsfeld $e_{\text {pot }}$ kann dabei gemäß Gleichung 37 als Funktion der Dichtedifferenz zwischen dem ungestörten Umgebungsfluid und der Wandoberfläche $\Delta \rho_{\infty \text {-W }}$ bestimmt werden. Eine Beschreibung der spezifischen kinetischen Energie $e_{\text {kin }}$ gelingt in Abhängigkeit der Fluiddichte $\rho_{\infty}$ und der Strömungsgeschwindigkeit $u$ gemäß Gleichung 38.

$$
\begin{aligned}
& e_{\mathrm{pot}}=g \cdot L \cdot\left(\rho_{\infty}-\rho_{\mathrm{W}}\right) \\
& e_{\mathrm{kin}}=\frac{\rho_{\infty}}{2} \cdot u^{2}
\end{aligned}
$$

Für die charakteristische Geschwindigkeit $u_{\text {char }}$ nach Gleichung 13 resultiert aus den Gleichungen 37 und 38 unmittelbar ein konstantes Verhältnis der potentiellen zur kinetischen Energie von $e_{\text {pot }} / e_{\text {kin }}=2$. In einer idealen (d.h. reibungsund trägheitsfreien) Strömung würde die von der wärmeübertragenden Oberfläche eingebrachte potentielle Energie hingegen vollständig in kinetische Energie umgewandelt werden, was gleichbedeutend mit einem Verhältnis von $e_{\text {pot }} / e_{\text {kin }}=1$ ist. Für Auftriebsströmungen in Luft wurden tatsächliche Werte von $e_{\text {pot }} / e_{\text {kin }}$ im stationären Fall mit 4,88 (vgl. [Kast 1972]) bis 5 (vgl. [Schlünder 1970]) ermittelt.

Zur Bestimmung des im instationären Fall als variabel angenommenen Verhältnisses von potentieller zu kinetischer Energie erscheint entsprechend den Gleichungen 37 und 38 insbesondere die örtliche und zeitliche Variation der Fluiddichte von zentraler Relevanz, da bei einer Beschleunigung der Auftriebsströmung durch das Gravitationsfeld Änderungen der Strömungsgeschwindigkeit $u$ ebenfalls durch Dichtevariationen initiiert werden. 


\subsubsection{Zeitliche Entwicklung der Wärmeübertragung durch freie Konvektion}

Im Umkehrschluss wird eine Änderung der mittleren Geschwindigkeit von instationären freien Konvektionsströmungen als direkte Auswirkung der Umwandlung von potentieller in kinetische Energie interpretiert. Daher wird für die analytische Prognose der zeitlichen Änderung der konvektiven Wärmeübertragung ergänzend der Zusammenhang zwischen der WärmeübertragungsIntensität und der Strömungsgeschwindigkeit betrachtet. Abbildung 5.1 zeigt dazu die dimensionslose konvektive Wärmeübertragung $N u_{\mathrm{x} / \mathrm{L}}$ gemäß den Gleichungen 8 und 9 in Abhängigkeit der charakteristischen Geschwindigkeit $u_{\text {char }}$ nach Gleichung 13 sowie als Funktion des Auftriebs-Volumenstroms $\dot{V}$ auf der Basis von empirisch ermittelten Korrelationsgleichungen (vgl. [Kriegel 1973]).

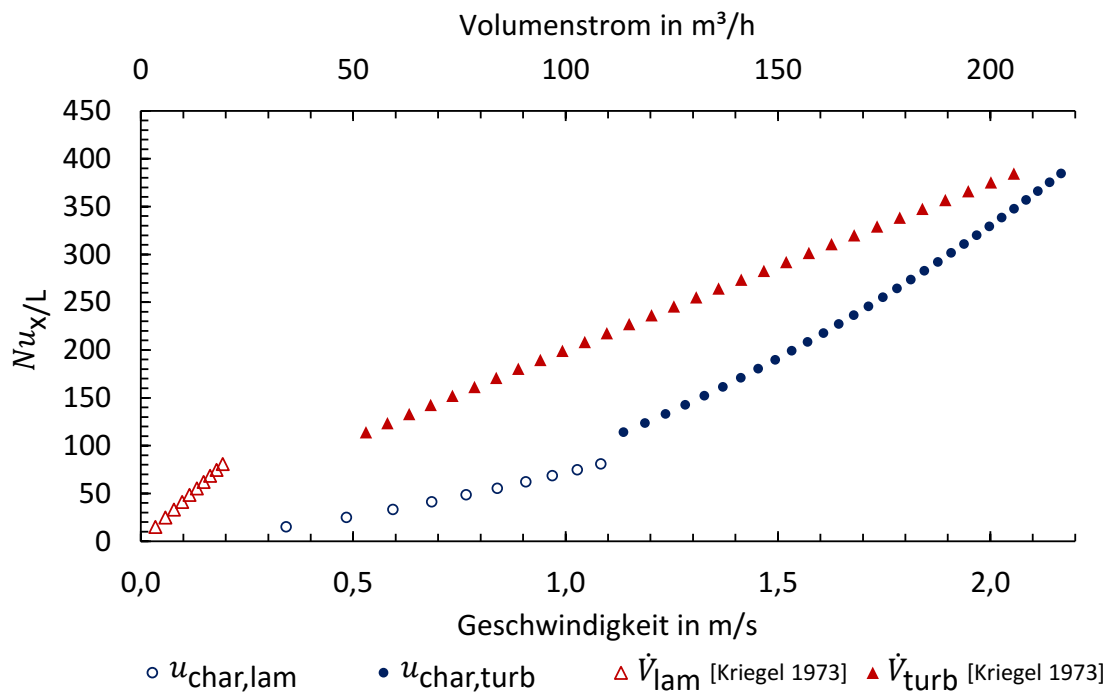

Abbildung 5.1: Konvektive Wärmeübertragung in Abhängigkeit der StrömungsIntensität

Für die dargestellten Strömungsgrößen $\left(G r_{\mathrm{L}}<5,5 \cdot 10^{10}\right)$ ist jeweils eine annähernd lineare Kopplung der konvektiven Wärmeübertragungs-Intensität erkennbar. Da für Geschwindigkeitsänderungen bei konstanter Schwerebeschleunigung ebenfalls von einer kontinuierlichen (d.h. zeitlich linearen) Entwicklung auszugehen ist, wird in den nachfolgend beschriebenen Prognosemo- 
dellen (siehe Kapitel 5.2 und 5.3) in erster Näherung auch die zeitliche Änderung der konvektiven Wärmeübertragungs-Intensität als linear betrachtet.

Sowohl die messtechnisch erfassten Momentanwerte der konvektiven Wärmeübertragung (vgl. Abbildung 3.22 und Abbildung 3.26), als auch die phänomenologischen Beobachtungen in den Kapiteln 4.1.2 und 4.2.2 lassen darüber hinaus das Maximum der instationären Wärmeübertragungs-Intensität zum Ende eines Wärmestrom-Impulses oder bei einer starken Reduzierung der zugeführten Wärmestromdichte erwarten.

\subsection{Prognosemodell zur instationären Wärmeübertragung bei zyklischer Variation der Randbedingungen}

Aus den in Kapitel 3.4 beschriebenen experimentellen Versuchsreihen wird deutlich, dass für die Prognose von Abweichungen zwischen der tatsächlichen konvektiven Wärmeübertragung innerhalb der instationären Phase und der quasi-stationären Betrachtungsweise neben dem zeitlichen Mittel der GrashofZahl weitere Einflussgrößen von Relevanz sind.

Für die grundlegende Gestaltung eines Regressionsmodells kommen daher als mögliche charakteristische Größen sowohl minimale, maximale und mittlere Werte sowie zeitliche Gradienten von Grashof-Zahlen, Wärmestromdichten und Temperaturdifferenzen, als auch diverse dimensionslose und -behaftete Parameter-Kombinationen in Betracht. Ein eindeutiger Kausalzusammenhang zeigt sich jedoch erst für den in Kapitel 5.1 vorgestellten physikalischen Ansatz. Abbildung 5.2 stellt dazu nochmals die Ergebnisse der Versuchsreihen aus Abbildung 3.24 dar, jedoch kategorisiert in Abhängigkeit der dimensionslosen Größe $\Pi^{*}$ entsprechend Gleichung 39.

$$
\Pi^{*}=\frac{\bar{\rho}_{\text {Zyklus,st }} \cdot \Delta t_{\text {Zyklus }}}{\rho_{\text {Impuls,st }} \cdot \Delta t_{\text {isph }}}
$$

In der Größe $\Pi^{*}$ werden die Fluiddichte, die sich für den Wärmestrom-Impuls im stationären Fall einstellen würde $\rho_{\text {Impuls,st }}$, dessen Pendant für den zeitlichen Mittelwert der Wärmezufuhr innerhalb der zyklischen Variation $\bar{\rho}_{\text {zyklus,st }}$ sowie die Dauer der instationären Phase $\Delta t_{\text {isPh }}$ und die gesamte Zyklusdauer $\Delta t_{\text {Zyklus }}$ ausgedrückt. 


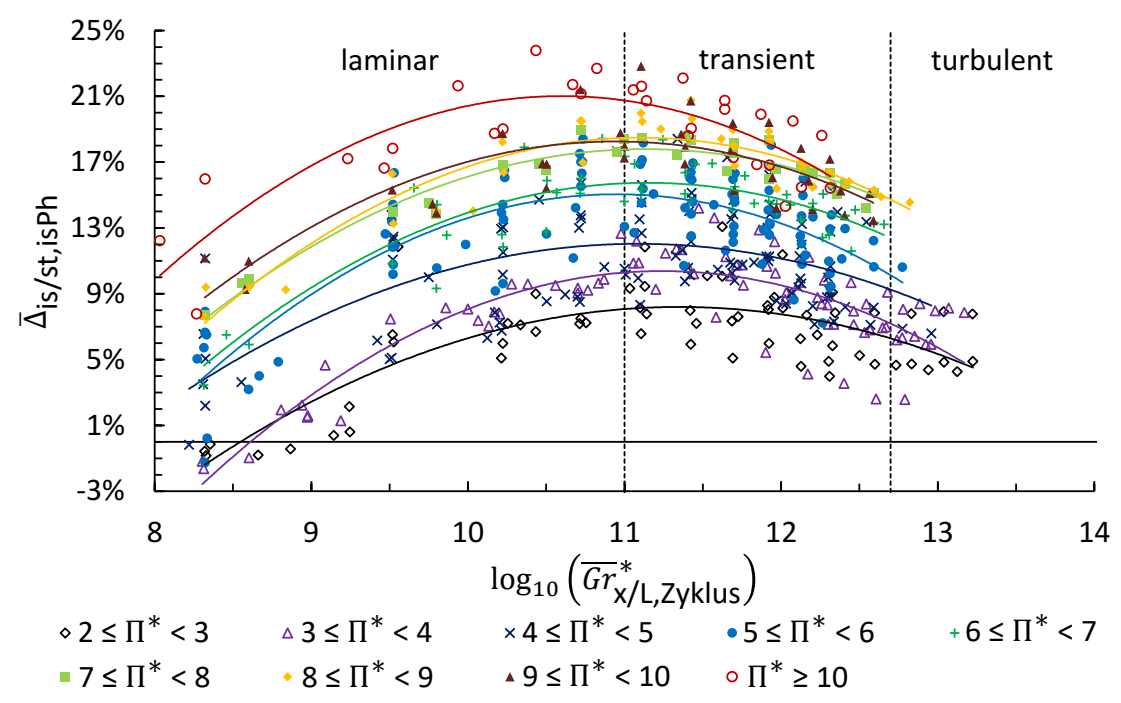

Abbildung 5.2: Zusätzliche konvektive Wärmeübertragung für $\Pi^{*}$-Kategorien

Für ansteigende Werte von $\Pi^{*}$ ist in Abbildung 5.2 auch bei gleicher mittlerer Grashof-Zahl $\overline{G r}_{\mathrm{x} / L, \text { Zyklus }}^{*}$ eine deutliche Tendenz zu größeren Abweichungen zwischen der im instationären Fall gemessenen Wärmeübertragung und der quasistationären Betrachtungsweise $\bar{\Delta}_{\text {is/st,isph }}$ zu erkennen. So zeigt sich für die jeweiligen quadratischen Regressionsfunktionen der $\Pi^{*}$-Kategorien eine deutlich ausgeprägte Verschiebung entlang der Ordinate.

Eine derartige Betrachtung von Impulsgrößen im Verhältnis zu mittleren Größen steht in prinzipieller Analogie zur Definition des Turbulenzgrades nach Gleichung 11. Gemäß der physikalischen Modellvorstellung aus Kapitel 5.1 wird $\Pi^{*}$ dabei als Maß für die Abweichung des mittleren Verhältnisses von potentieller zu kinetischer Energie innerhalb der instationären Phase vom Verhältnis $e_{\text {pot }} / e_{\text {kin }}$ im stationären Fall interpretiert.

\subsubsection{Validierungsmessungen für zyklische Variationen}

Zur Überprüfung einer direkten Abhängigkeit zwischen den festgestellten Auswirkungen von instationären Strömungsstrukturen auf die konvektive Wärmeübertragung und der Größe $\Pi^{*}$ nach Gleichung 39 werden in Ergänzung zu den Versuchsreihen aus Kapitel 3.4.1 insgesamt 17 Validierungsmessungen mit zyk- 
lisch variierten Randbedingungen durchgeführt. Die Definition der VariationsRandbedingungen erfolgt dabei anhand der zu erwartenden Verhältnisse von Impulsgrößen zu mittleren Größen, sodass eine möglichst große Bandbreite von $\Pi^{*}$ berücksichtigt wird. So werden $\Pi^{*}$-Werte zwischen 2 und ca. 10,8 betrachtet, wobei die Untersuchungs-Schrittweite bei ca. $\Delta \Pi^{*} \approx 0,5$ liegt. Zur Reduktion der zufälligen Messunsicherheits-Anteile der in Kapitel 3.3.4 aufgeführten messtechnischen Gesamtunsicherheit sowie zur Überprüfung der Reproduzierbarkeit der Ergebnisse werden sämtliche Validierungsmessungen jeweils fünffach wiederholt.

Abbildung 5.3 zeigt die arithmetischen Mittel der resultierenden Abweichungen zwischen der im instationären Fall gemessenen konvektiven Wärmeübertragung und der quasi-stationären Betrachtung $\bar{\Delta}_{\text {is/st,isph }}$ für die 17 Validierungsmessungen sowie die jeweilige Standardabweichung $s$.

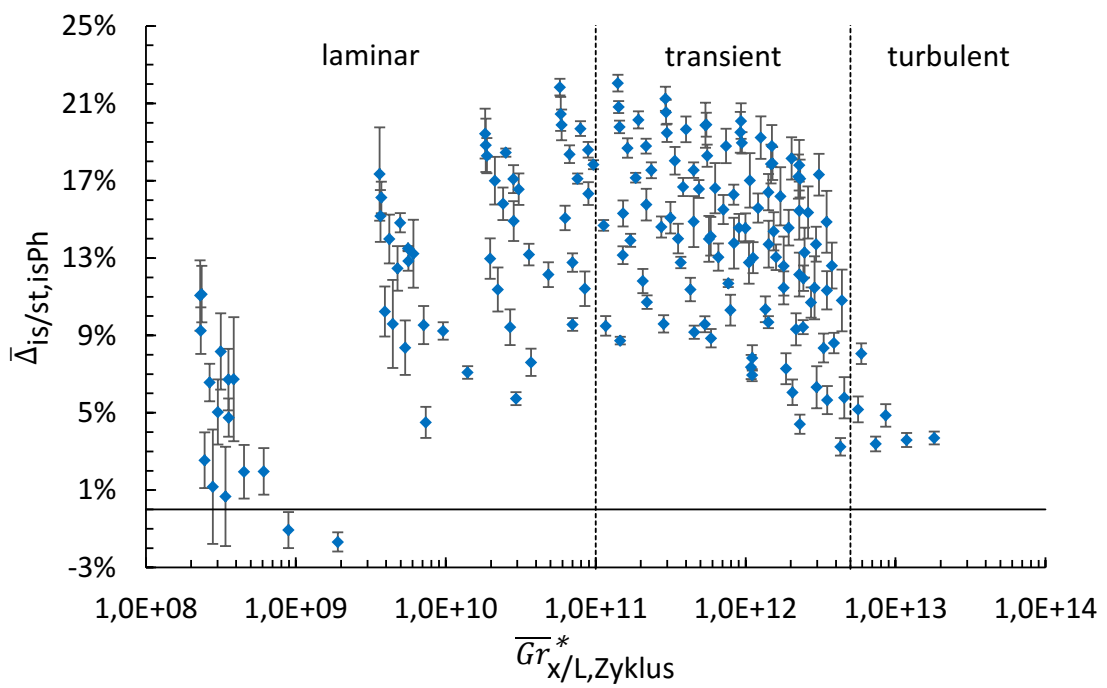

Abbildung 5.3: Ergebnisübersicht der Wiederholungsmessungen zu zyklischen Variationen

Die festgestellte Standardabweichung der fünffachen Wiederholungsmessungen liegt dabei zwischen $0,1 \% \leq s \leq 3,2 \%$, ihr arithmetisches Mittel für alle 170 Einzelergebnisse beträgt $s_{\mathrm{m}}=0,9 \%$. In Analogie zur MessunsicherheitsFortpflanzung (vgl. Kapitel 3.3.4) ist dabei eine Tendenz zu großen relativen Ge- 
samtunsicherheiten bei geringen Grashof-Zahlen $\overline{G r}_{\text {x/L,Zyklus }}^{*}$ festzustellen. Erhöhte Standardabweichungen zeigen sich außerdem im Übergangsbereich (bei ca. $5 \cdot 10^{11}<\overline{G r}_{x / L, \text { zyklus }}^{*}<10^{13}$ ).

Abbildung 5.4 zeigt die zugehörigen Abweichungen der instationären konvektiven Wärmeübertragung der Validierungsmessungen gegenüber der quasistationären Betrachtungsweise $\bar{\Delta}_{\text {is/st,isph }}$ in Abhängigkeit des Verhältnisses von Impulsgrößen zu mittleren Größen $\Pi^{*}$.

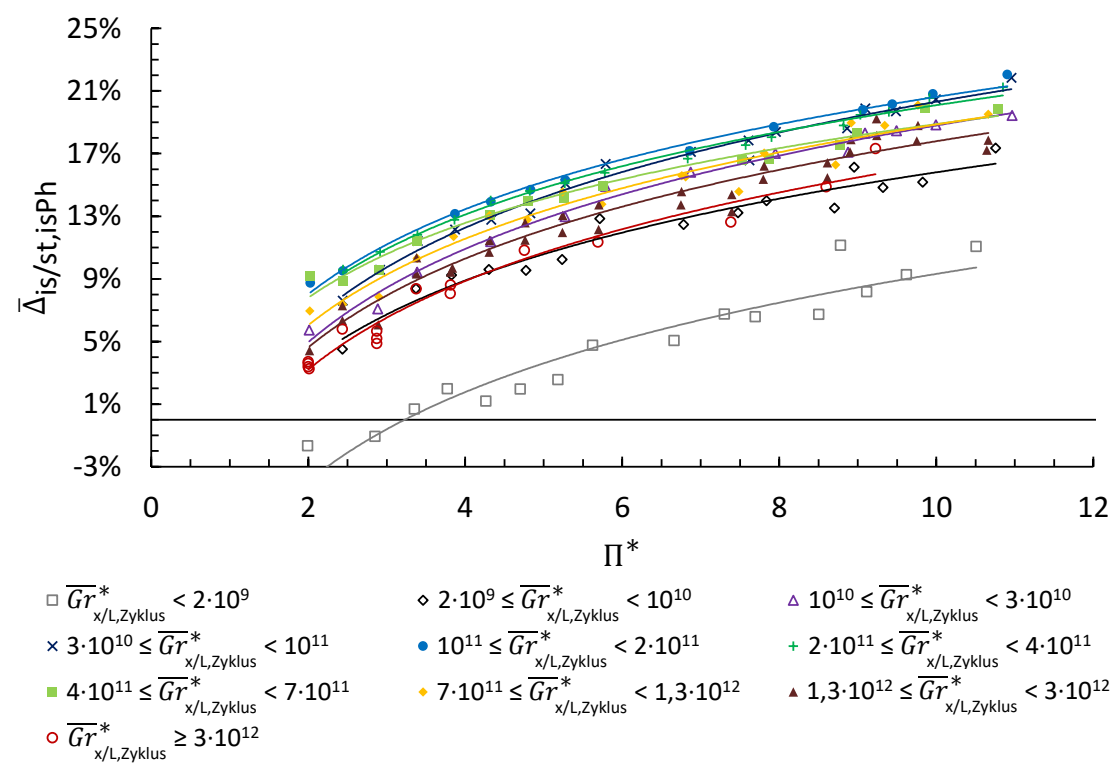

Abbildung 5.4: Zusätzliche konvektive Wärmeübertragung als Funktion von $\Pi^{*}$

Für ähnliche mittlere Grashof-Zahlen $\overline{G r}_{\mathrm{x} / L, \text { Zyklus }}^{*}$ ist dabei in Analogie zu Abbildung 5.2 für zunehmende $\Pi^{*}$ ein monotoner Anstieg des Einflusses der instationären Betriebsweise $\bar{\Delta}_{\text {is/st,isph }}$ zu erkennen.

Zur Prognose der Abweichungen zwischen der tatsächlichen konvektiven Wärmeübertragung innerhalb der instationären Phase einer zyklischen Variation der Randbedingungen und der quasi-stationären Betrachtungsweise $\bar{\Delta}_{\mathrm{is} / \mathrm{st} \text {,isPh }}$ wird daher ein logarithmisches Regressionsmodell entsprechend Gleichung 40 vorgeschlagen. 


$$
\bar{\Delta}_{\mathrm{is} / \mathrm{st}, \mathrm{isPh}}=m \cdot \ln \left(\Pi^{*}\right)+b
$$

Für den Regressionsparameter $m$ liegt im Falle der zyklisch variierten Randbedingungen ein konstanter Wert von ca. 0,0798 vor, während $b$ eine Abhängigkeit von $\overline{G r}_{\mathrm{x} / L, \text { Zyklus }}^{*}$ entsprechend Gleichung 41 aufweist.

$$
b=\left\{\begin{array}{cl}
0,0208 \cdot \ln \left(\overline{G r}_{\text {x/L,Zyklus }}^{*}\right)-0,4974 & \text { für } \overline{G r}_{\text {x/L,Zyklus }}^{*} \leq 10^{11} \\
-0,0129 \cdot \ln \left(\overline{G r}_{\text {x/L,Zyklus }}^{*}\right)+0,3591 & \text { für } \overline{G r}_{\text {x/L,Zyklus }}^{*}>10^{11}
\end{array}\right.
$$

Eine umfangreiche Ergebnisübersicht der Validierungsmessungen bei zyklisch variierten Strömungs-Randbedingungen ist zudem in Anhang A.1 aufgeführt.

\subsubsection{Instationäre Phase bei zyklischen Variationen}

Für die Dauer der instationären Phase $\Delta t_{\text {isPh }}$, innerhalb der die Momentanwerte der konvektiven Wärmeübertragung $\overline{\dot{q}}_{\mathrm{c}, \mathrm{is}, 90 \mathrm{~s}}$ entsprechend Gleichung $31 \mathrm{um}$ mehr als ein Prozent vom quasi-stationären Vergleichswert $\overline{\dot{q}}_{\mathrm{c}, \mathrm{st}, 90 \mathrm{~s}}$ abweichen, zeigt sich eine Abhängigkeit von der Dauer des jeweiligen WärmestromImpulses $\Delta t_{\text {Impuls }}$ und der Zyklus-Gesamtdauer $\Delta t_{\text {Zyklus }}$ gemäß Gleichung 42 .

$$
\frac{\Delta t_{\text {isph }}}{\Delta t_{\text {Zyklus }}}=7,0716 \cdot\left(\frac{\Delta t_{\text {Impuls }}}{\Delta t_{\text {Zyklus }}^{2}}\right)^{0,3563}
$$

Abbildung 5.5 zeigt die zugrunde liegenden Regressionsdaten der Validierungsmessungen aus Kapitel 5.2.1. Die Dauer der instationären Phase variiert bei einem arithmetischen Mittel von $\Delta t_{\text {isph,m }}=24 \%$ zwischen $13 \% \leq$ $\Delta t_{\text {isph }} \leq 53 \%$ und steht somit in sehr guter Übereinstimmung zu den Versuchsreihen in Kapitel 3.4.1.

Gemäß der in Kapitel 5.1 .1 beschriebenen physikalischen Modellvorstellung erscheint für die instationäre Wärmeübertragung insbesondere die Änderung der Fluiddichte von Relevanz. Dabei liegt zunächst die Vermutung nahe, dass auch ein unmittelbarer Zusammenhang zwischen der Dauer der instationären Phase und dem zeitlichen Gradienten der Oberflächentemperatur an der wärmeübertragenden Platte besteht. Letzterer wird maßgeblich durch die zugeführte Wärmestromdichte, die Wärmekapazität der Platte und durch die aktuelle Wärmeabfuhr selbst geprägt (vgl. Gleichung 29). 


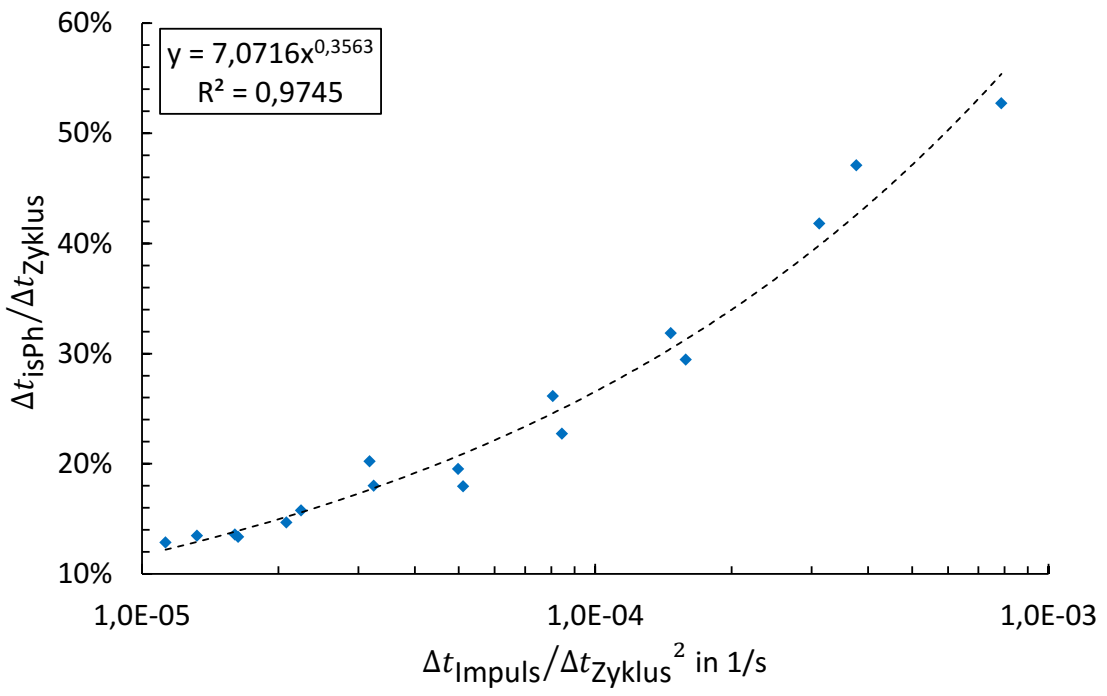

Abbildung 5.5: Dauer der instationären Phase bei zyklischen Variationen

Dementsprechend werden Versuchsreihen mit sehr unterschiedlichen zeitlichen Gradienten der Oberflächentemperatur durchgeführt. Dabei treten wäh-

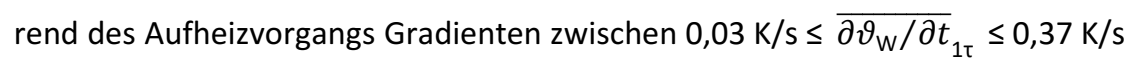
auf und während des Abkühlens Werte zwischen $0,01 \mathrm{~K} / \mathrm{s} \leq$

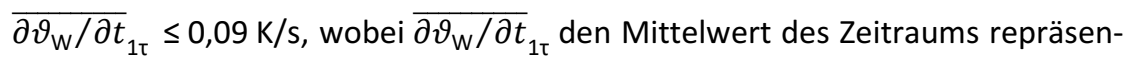
tiert, in dem $63,2 \%$ der Temperaturänderung $\Delta \vartheta_{\mathrm{W}}$ des Zyklus vollzogen werden $(1 \tau)$. Für die Dauer der instationären Phase $\Delta t_{\text {isph }}$ zeigt sich jedoch keinerlei Abhängigkeit vom zeitlichen Temperaturgradienten ${\overline{\partial \vartheta_{\mathrm{w}} / \partial t}}_{1 \mathrm{r}}$. In Abbildung 5.6 ist dazu die Dauer der instationären Phase $\Delta t_{\text {isPh }}$ für zwei unterschiedliche Impuls-Wärmestromdichten $\dot{q}_{\text {Impuls }}$ bei gleicher Impulsdauer $\left(\Delta t_{\text {Impuls }}=120 \mathrm{~s}\right)$ und gleichem Auskühlzeitraum $\left(\Delta t_{\text {Auskühlung }}=1.800 \mathrm{~s}\right)$ dargestellt. Für den Wärmestromimpuls von $\dot{q}_{\text {Impuls }}=356 \mathrm{~W} / \mathrm{m}^{2}$ liegt während der Aufheizphase ein zeitlicher Oberflächentemperaturgradient von ${\overline{\partial \vartheta_{\mathrm{W}} / \partial t_{1 \tau}}}_{1 \mathrm{c}}=0,09 \mathrm{~K} / \mathrm{s}$ vor; bei $\dot{q}_{\text {Impuls }}=1.430 \mathrm{~W} / \mathrm{m}^{2}$ sind dies ${\overline{\partial \vartheta_{\mathrm{W}} / \partial t_{1 \tau}}}_{1 \mathrm{~s}}=0,36 \mathrm{~K} / \mathrm{s}$. Unabhängig von der Intensität der Abweichung der instationären Wärmeübertragung von der quasistationären Betrachtungsweise $\bar{\Delta}_{\text {is/st,isph }}$ zeigt sich die Dauer der instationären Phase dabei mit $\Delta t_{\text {isph }}=286 \mathrm{~s}$ bei $\dot{q}_{\text {Impuls }}=356 \mathrm{~W} / \mathrm{m}^{2}$ und $\Delta t_{\text {isPh }}=282 \mathrm{~s}$ bei 
$\dot{q}_{\text {Impuls }}=1.430 \mathrm{~W} / \mathrm{m}^{2}$ vor dem Hintergrund eines Messintervalls von $2 \mathrm{~s}$ als nahezu identisch.

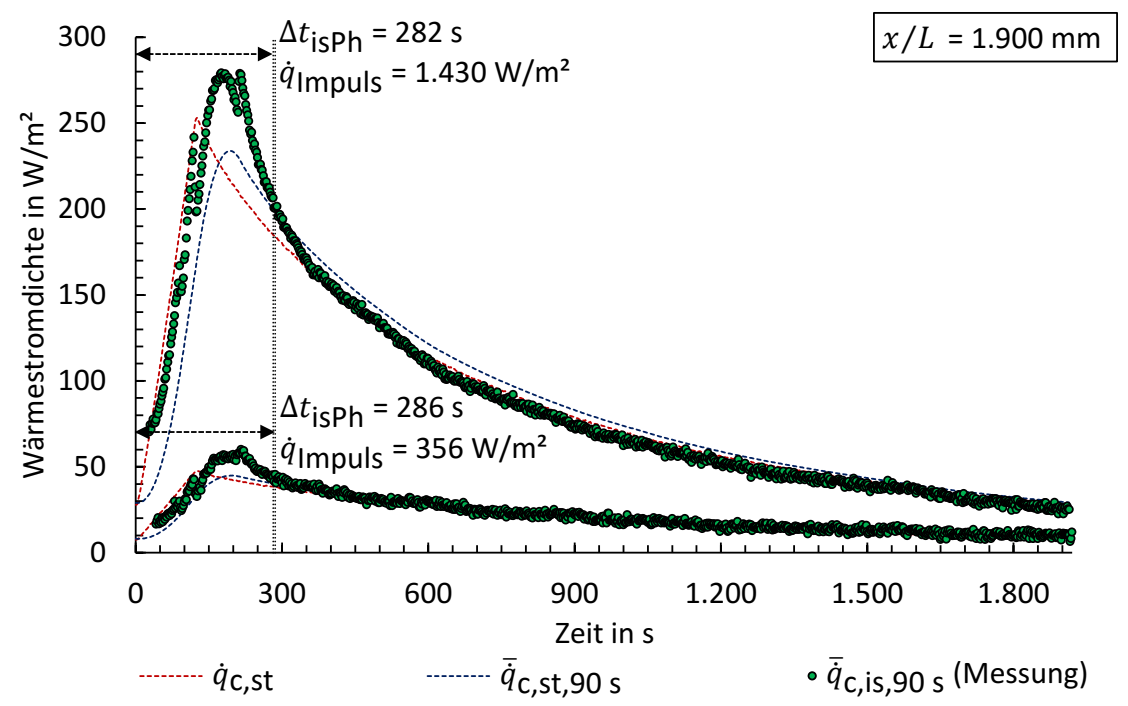

Abbildung 5.6: Dauer der instationären Phase bei variabler Wärmestromdichte (mit $\Delta t_{\text {Impuls }}=120 \mathrm{~s}$ und $\Delta t_{\text {Auskühlung }}=1.800 \mathrm{~s}$ )

Für zyklische Variationen der Randbedingungen ist daher von einer Unabhängigkeit der Regressionsfunktion nach Gleichung 42 von der thermischen Kapazität der beheizten Platte und der Impuls-Wärmestromdichte auszugehen.

Ferner verhält sich die Dauer der instationären Phase unabhängig von der mittleren Grashof-Zahl. So weisen alle betrachteten Strömungs-Teilgebiete (vgl. Kapitel 1.1.1) einer Versuchsreihe stets den gleichen Anteil der instationären Phase an der gesamten Zyklusdauer auf.

Der in Kapitel 5.1 vorgestellten physikalischen Modellvorstellung folgend, wird die beobachtete (ausschließliche) Abhängigkeit der Dauer der instationären Phase von der Impulsdauer und der gesamten Zyklusdauer derart interpretiert, dass für die Dauer des Wärmestrom-Impulses ein Aufbau des Überschusses an potentieller Energie im Fluid stattfindet und dieser darüber hinaus kontinuierlich wieder abgebaut wird. Ein Gedankenexperiment, bei dem nach dem Ende des Wärmestrom-Impulses plötzlich die beheizte Oberfläche aus dem Fluid entfernt wird, verdeutlicht ferner, dass der darauffolgende Ausgleichsvorgang zwi- 
schen potentieller und kinetischer Energie lediglich eine Funktion des Fluids und der Zeit sein muss.

Aufgrund des medienspezifischen Verhältnisses von Impuls- und Wärmetransport liegt für die Dauer der instationären Phase jedoch eine Fluid-Abhängigkeit, also ein Einfluss der Prandtl-Zahl nahe. So erscheint die Temperaturleitfähigkeit $a$ insbesondere für den Aufbau des Überschusses an potentieller Energie von Relevanz, während für den anschließenden Ausgleichsvorgang von einem besonderen Einfluss der kinematischen Viskosität $v$ auszugehen ist.

\subsubsection{Momentanwerte von zyklischen Variationen}

Entsprechend der in Kapitel 5.1.2 dargestellten Annahme einer linearen zeitlichen Zuwachs- und Abklingrate der Intensität der konvektiven Wärmeübertragung wird zur Bestimmung der Momentanwerte innerhalb der instationären Phase ein vereinfachtes lineares Berechnungsmodell vorgeschlagen. Darin wird die mittlere Abweichung der instationären konvektiven Wärmeübertragung von der quasi-stationären Betrachtungsweise $\bar{\Delta}_{\text {is/st,isph }}$ gemäß Gleichung 33 so auf die Dauer der instationären Phase $\Delta t_{\text {isph }}$ verteilt, dass für die Momentanwerte $\Delta_{\text {is/st }}$ vom Beginn eines jeweiligen Zyklus ein kontinuierlicher zeitlicher Zuwachs bis zum Ende des Wärmestrom-Impulses $\Delta t_{\text {Impuls }}$ resultiert. Vom dort befindlichen Maximum $\Delta_{\text {is/st,max }}$ wird wiederum ein kontinuierliches Abklingen der Momentanwerte der (zusätzlich durch die instationäre Betriebsweise verursachten) Wärmeübertragungs-Intensität $\Delta_{\text {is/st }}$ bis zum Ende der instationären Phase $\Delta t_{\text {isph }}$ angenommen, wie in Abbildung 5.7 schematisch dargestellt.

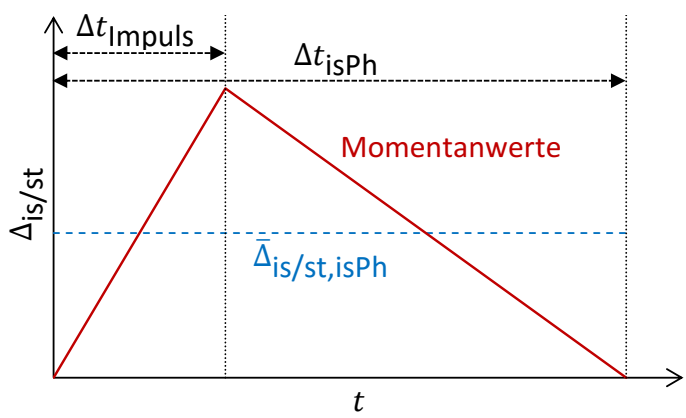

Abbildung 5.7: Prognose-Modell zur instationären konvektiven Wärmeübertragung 
Der beschriebene zeitliche Verlauf wird entsprechend durch Gleichung 43 ausgedrückt.

$$
\Delta_{\mathrm{is} / \mathrm{st}}=\left\{\begin{array}{cc}
\frac{2 \cdot \bar{\Delta}_{\mathrm{is} / \mathrm{st}, \mathrm{sph}}}{\Delta t_{\mathrm{Impuls}}} \cdot t & \text { für } t \leq \Delta t_{\text {Impuls }} \\
2 \cdot \bar{\Delta}_{\mathrm{is} / \mathrm{st}, \mathrm{isPh}}-\left[\frac{2 \cdot \bar{\Delta}_{\mathrm{is} / \mathrm{t} \mathrm{isph}}}{\Delta t_{\mathrm{isph}}-\Delta t_{\mathrm{Impuls}}} \cdot\left(t-\Delta t_{\text {Impuls }}\right)\right] & \text { für } t>\Delta t_{\text {Impuls }}
\end{array}\right.
$$

Die insgesamt prognostizierte konvektive Wärmeübertragungs-Intensität des instationären Falls $\dot{q}_{\mathrm{c} \text {,is }}$ resultiert schließlich für jeden Zeitpunkt gemäß Gleichung 44 .

$$
\dot{q}_{\mathrm{c}, \mathrm{is}}=\dot{q}_{\mathrm{c}, \mathrm{st}}+\dot{q}_{\mathrm{c}, \mathrm{st}} \cdot \Delta_{\mathrm{is} / \mathrm{st}}
$$

Darin repräsentiert $\dot{q}_{\mathrm{c}, \text { st }}$ den quasi-stationären Momentanwert der konvektiven Wärmestromdichte entsprechend Kapitel 3.3.1. Für beliebige Randbedingungen kann $\dot{q}_{c, s t}$ alternativ aus den Gleichungen 2 bis 10 ermittelt werden.

Abbildung 5.8 zeigt die Versuchsreihe aus Abbildung 3.22 nochmals, jedoch ergänzt um die Prognose der Momentanwerte der instationären konvektiven Wärmeübertragung $\dot{q}_{\mathrm{c}, \text { is }}$ gemäß Gleichung 44 .

In Analogie zu Kapitel 3.4.1 handelt es sich bei den in Grün dargestellten, gemessenen Momentanwerten $\overline{\dot{q}}_{\mathrm{c}, \mathrm{is}, 90 \mathrm{~s}}$ jeweils um gleitende Mittelwerte der letzten 90 s. Ein direkter Vergleich zu dem in Blau dargestellten Pendant der prognostizierten Momentanwerte $\overline{\dot{q}}_{c, i s, 90 \text { s }}$ zeigt eine sehr gute Übereinstimmung für den gesamten zeitlichen Verlauf des betrachteten Zyklus.

Für eine derartige Prognose der Momentanwerte der konvektiven Wärmeübertragung innerhalb der instationären Phase zeigt sich darüber hinaus für sämtliche der durchgeführten Versuchsreihen mit zyklisch variierten Randbedingungen eine gute grafische Übereinstimmung zu den gemessenen Momentanwerten. Lediglich für sehr kurze Impulsdauern $\left(\Delta t_{\text {Impuls }} \leq 60 \mathrm{~s}\right)$ übersteigen die gemessenen Momentanwerte die Prognose zum Zeitpunkt des Impulsendes nennenswert. Aufgrund des in Kapitel 3.3.2 beschriebenen Mittelungs-Intervalls von $90 \mathrm{~s}$ zur Messung von Momentanwerten der konvektiven Wärmeübertragung ist für Versuchsreihen mit Impulsdauern $\Delta t_{\text {Impuls }}<90 \mathrm{~s}$ jedoch eher von einer eingeschränkten Aussagekraft der gemessenen Momentanwerte auszugehen. 


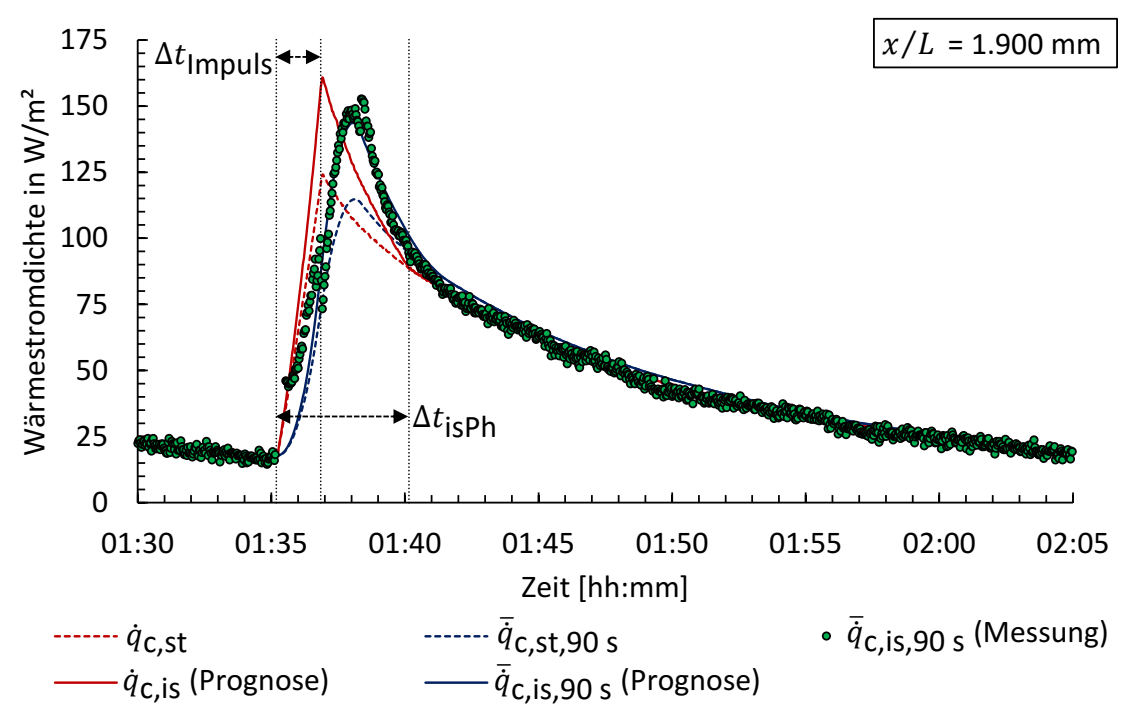

Abbildung 5.8: Momentanwert-Prognose für zyklische Variationen (mit $\dot{q}_{\text {Impuls }}=913 \mathrm{~W} / \mathrm{m}^{2}, \Delta t_{\text {Impuls }}=102 \mathrm{~s}$ und $\Delta t_{\text {Auskühlung }}=1.800 \mathrm{~s}$ )

\subsubsection{Modellanwendung und Prognosegüte für zyklische Variationen}

Für das in den Gleichungen 39 bis 44 vorgestellte Modell zur Prognose der instationären konvektiven Wärmeübertragung bei zyklisch variierten StrömungsRandbedingungen zeigt Abbildung 5.9 eine schematische Übersicht der zugrunde liegenden Vorgehensweise.

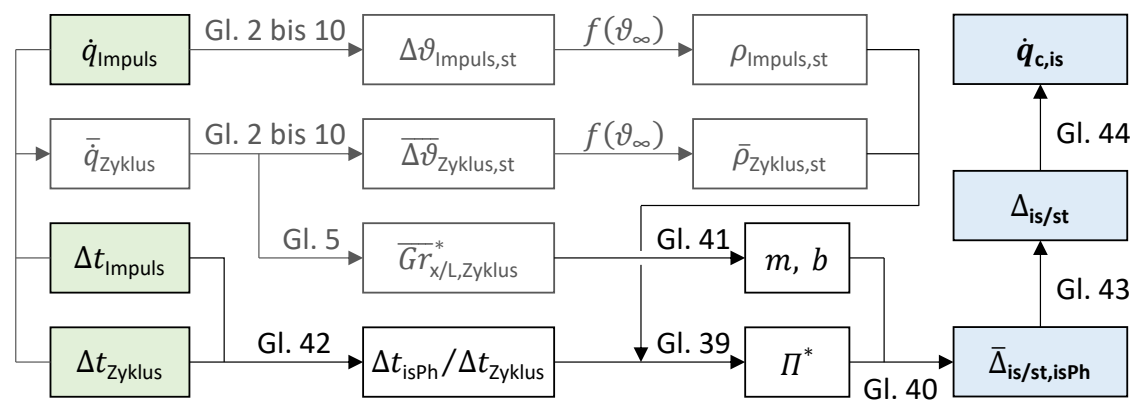

Abbildung 5.9: Anwendungsschema des Regressionsmodells für zyklische Variationen 
Dabei stellen die Wärmestromdichte $\dot{q}_{\text {Impuls }}$ und die Dauer des Aufheizimpulses $\Delta t_{\text {Impuls }}$ sowie die gesamte Zyklusdauer $\Delta t_{\text {zyklus }}$ die bekannten Eingangsgrößen dar. Mit Hilfe der etablierten Berechnungskorrelationen (vgl. Kapitel 1.1) werden zunächst die Temperaturdifferenzen $\Delta \vartheta_{\text {Impuls,st }}$ und $\overline{\Delta \vartheta}_{\text {zyklus,st }}$ ermittelt, die sich im stationären Fall für den Impulswärmestrom $\dot{q}_{\text {Impuls }}$ und den mittleren Wärmestrom $\dot{q}_{\text {zyklus }}$ einstellen würden. Die zugehörigen mittleren Fluiddichten $\rho_{\text {Impuls,st }}$ und $\bar{\rho}_{\text {Zyklus,st }}$ werden im Anschluss mit der Dauer der instationären Phase $\Delta t_{\text {isPh }}$ in der dimensionslosen Kennzahl $\Pi^{*}$ ausgedrückt. Zusammen mit den an das zeitliche Mittel der Grashof-Zahl $\overline{G r}_{\text {x/L,Zyklus }}^{*}$ angepassten Regressionsparametern $m$ und $b$ folgt daraus die mittlere Abweichung der konvektiven Wärmeübertragungs-Intensität des instationären Falles von der quasistationären Betrachtungsweise innerhalb der instationären Phase $\bar{\Delta}_{\text {is/st,isPh. Die }}$ mittlere Abweichung $\bar{\Delta}_{\text {is/st,isph }}$ kann durch einen charakteristischen zeitlichen Verlauf in Momentanwerten $\Delta_{\text {is/st }}$ ausgedrückt werden. Durch Addition der quasi-stationären Vergleichswerte resultiert schließlich die Prognose der für jeden Zeitpunkt insgesamt vorhandenen konvektiven WärmeübertragungsIntensität im instationären Fall $\dot{q}_{\mathrm{c}, \text { is }}$.

Abbildung 5.10 zeigt einen Vergleich zwischen den Berechnungsergebnissen des analytischen Regressionsmodells und den jeweiligen Validierungsmessungen des Kapitels 5.2.1.

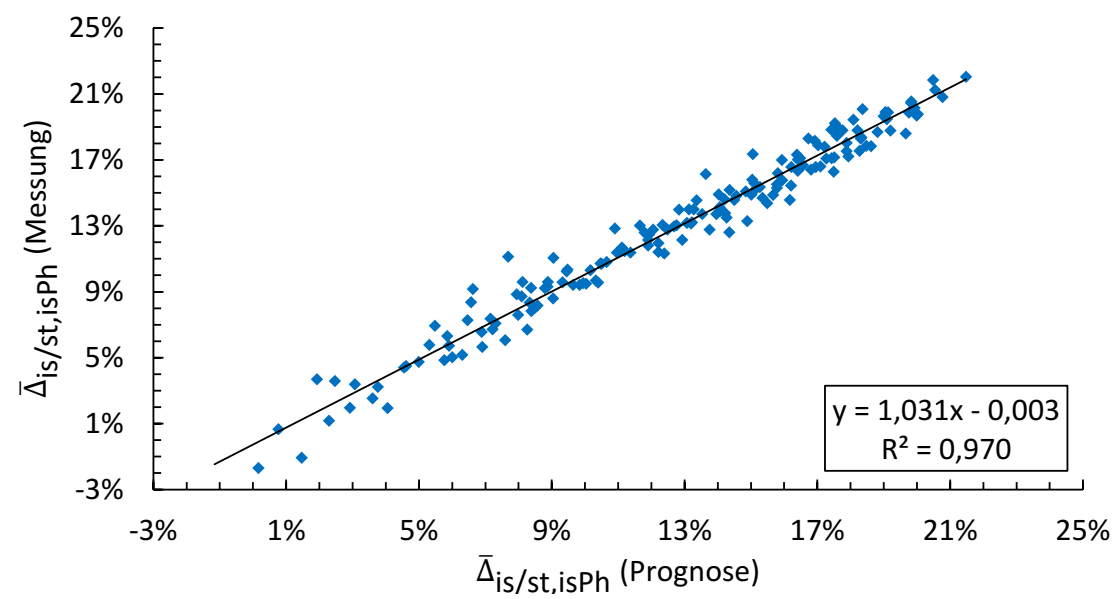

Abbildung 5.10: Prognosegüte des Regressionsmodells für zyklische Variationen 
Wie aus der Anordnung der Vergleichspunkte ersichtlich wird, zeigt sich dabei insgesamt eine gute Übereinstimmung. So beträgt die Differenz zwischen Prognose und Messwerten für die 170 Einzelergebnisse der Validierungsmessungen zwischen $0 \%$ und maximal 3,5\%, das arithmetische Mittel beläuft sich auf $0,7 \%$. Zwischen dem Ausmaß der Prognosefehler und den Regressionsparametern $\Pi^{*}$ oder $\overline{G r}_{\mathrm{x} / L, \text { Zyklus }}^{*}$ ist dabei keine eindeutige Kausalität erkennbar. Die größten Differenzen treten jedoch entsprechend der Gesamtunsicherheit (vgl. Kapitel 5.2.1) bei sehr kleinen Grashof-Zahlen $\left(\overline{G r}_{\text {x/L,Zyklus }}^{*}<10^{10}\right)$ auf.

\subsection{Prognosemodell zur instationären Wärmeübertragung bei sprungartigen Änderungen mit anschließender Beharrung}

Für die Prognose der konvektiven Wärmeübertragung nach einer sprungartigen Änderung der Strömungs-Randbedingungen mit anschließender Beharrung wird im Grundsatz der gleiche Regressionsansatz vorgeschlagen, wie im Falle der zyklischen Variationen (vgl. Kapitel 5.2). Im Unterschied dazu existiert jedoch keine definierte Zyklusdauer $\Delta t_{\text {Zyklus, }}$, weshalb ersatzweise der Zeitraum vom Beginn der Wärmestromzufuhr bis zum Erreichen des Oberflächentemperatur-Sollwertes $\Delta t_{\text {Sprung }}$ herangezogen wird. Ferner beziehen sich die Angaben zu mittleren Größen anstelle einer zeitlichen Mittelung auf das arithmetische Mittel der Oberflächentemperatur vor und nach dem Sprung $\vartheta_{\mathrm{W}, \mathrm{m}}$. Der resultierende Regressionsparameter $\Pi$ wird durch Gleichung 45 ausgedrückt.

$$
\Pi=\frac{\rho_{\mathrm{m}, \mathrm{st}} \cdot \Delta t_{\mathrm{isph}}}{\rho_{\mathrm{max}, \mathrm{st}} \cdot \Delta t_{\mathrm{sprung}}}
$$

Abbildung 5.11 zeigt dazu die entsprechende Kategorisierung der Messdaten aus Abbildung 3.27 in Abhängigkeit der dimensionslosen Größe $\Pi$. In Analogie zu Abbildung 5.2 ist mit zunehmenden Werten von $\Pi$ bei gleicher mittlerer Grashof-Zahl $G r_{\mathrm{x} / L, \mathrm{~m}}$ eine kontinuierliche Verschiebung der Regressionsfunktionen für $\bar{\Delta}_{\mathrm{is} / \mathrm{st} \text {,isph }}$ entlang der Ordinate zu erkennen. Aufgrund des geringeren Stichprobenumfangs der sprungartigen Randbedingungs-Variationen (vgl. Kapitel 3.4.2) wird im Vergleich zu Kapitel 5.2 eine entsprechend gröbere Kategorisierung vorgenommen. 


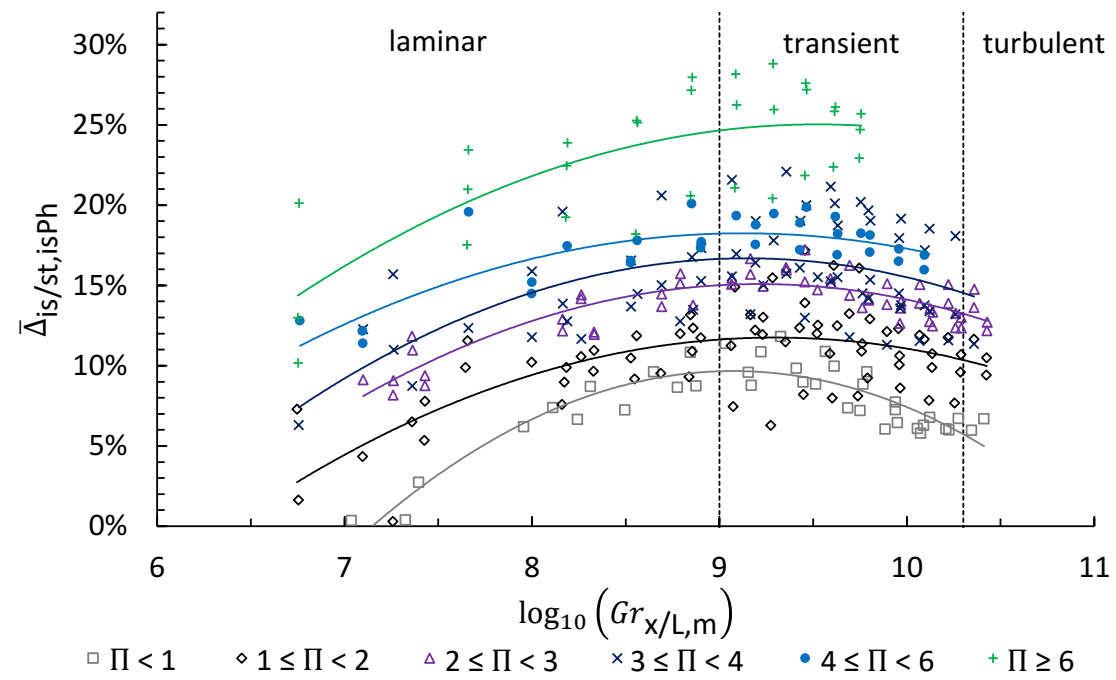

Abbildung 5.11: Zusätzliche konvektive Wärmeübertragung für $\Pi$-Kategorien

\subsubsection{Validierungsmessungen für sprungartige Änderungen}

Zur Bestimmung der Regressionsparameter für ein Prognosemodell der instationären konvektiven Wärmeübertragung bei einer sprungartigen Variation der Randbedingungen werden elf Validierungsmessungen durchgeführt, die gemäß der in Kapitel 1.1.1 beschriebenen Bilanzierung von Strömungs-Teilgebieten zu einer Regressions-Grundlage von insgesamt 110 Einzelergebnissen führen.

Betrachtet werden dabei $\Pi$-Werte zwischen etwa 0,8 und ca. 11,4. Die Untersuchungs-Schrittweite beträgt ca. $\Delta \Pi \approx 1$. Zu Zwecken der Reduktion von zufälligen Messunsicherheiten und zur Überprüfung der Reproduzierbarkeit der Ergebnisse werden die Validierungsmessungen für sprungartige RandbedingungsVariationen ebenfalls fünffach wiederholt.

Die so erfassten Unterschiede zwischen instationärer Wärmeübertragung und dem quasi-stationären Vergleichsfall $\bar{\Delta}_{\text {is/st,isph }}$ sowie deren Standardabweichungen $s$ sind für sprungartige Änderungen mit anschließender Beharrung in Abbildung 5.12 dargestellt. Die Standardabweichung bewegt sich dabei zwischen $0,3 \% \leq s \leq 3,4 \%$, der Mittelwert beträgt $s_{\mathrm{m}}=1,2 \%$. Auch hier zeigen sich die größten relativen Unsicherheiten bei geringen Grashof-Zahlen $\left(G r_{\mathrm{x} / \mathrm{L}, \mathrm{m}}<10^{8}\right)$ und im transienten Übergangsbereich (ca. $10^{9}<G r_{\mathrm{x} / \mathrm{L}, \mathrm{m}}<2 \cdot 10^{11}$ ). 


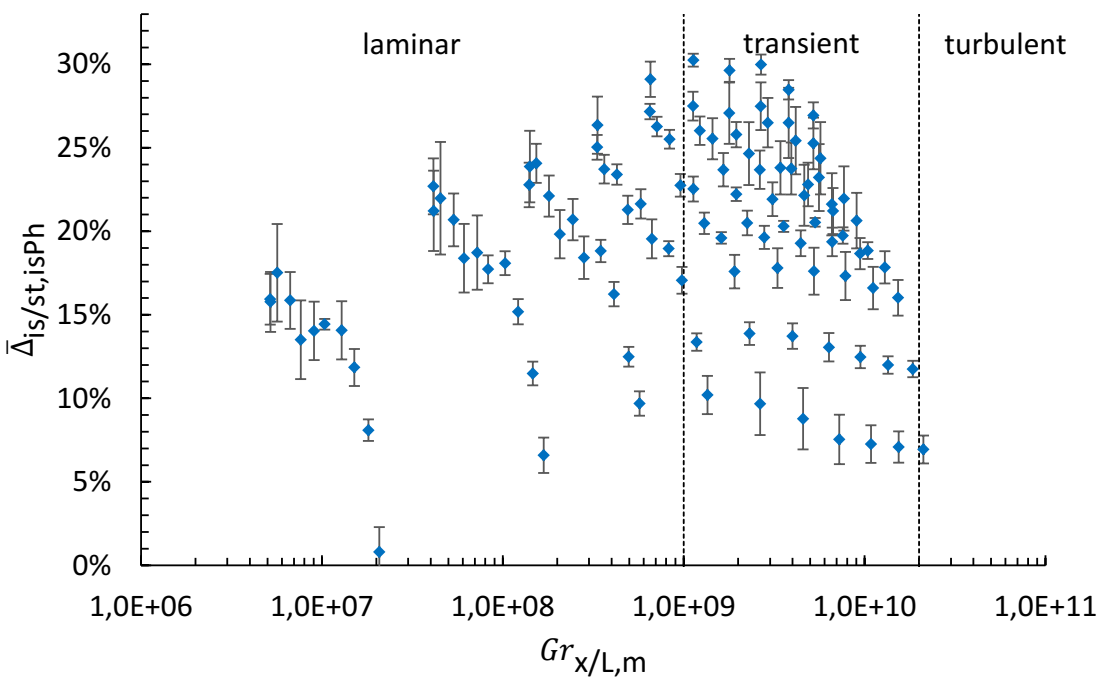

Abbildung 5.12: Ergebnisübersicht der Wiederholungsmessungen zu sprungartigen Änderungen

Die Beeinflussung der konvektiven Wärmeübertragung durch eine instationäre

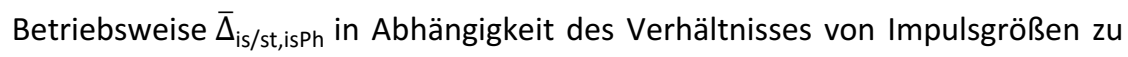
mittleren Größen $\Pi$ zeigt Abbildung 5.13. Für ähnliche mittlere Grashof-Zahlen $G r_{\mathrm{x} / L, \mathrm{~m}}$ wird dabei erneut eine direkte Korrelation zur Größe $\Pi$ deutlich.

Zur Prognose der instationären konvektiven Wärmeübertragung bei sprungartig veränderten Oberflächentemperaturen an der vertikalen Platte wird daher ebenfalls ein logarithmisches Regressionsmodell entsprechend Gleichung 46 vorgeschlagen.

$$
\bar{\Delta}_{\mathrm{is} / \mathrm{st}, \mathrm{isPh}}=m \cdot \ln (\Pi)+b
$$

Der Regressionsparameter $m$ resultiert dabei mit einem konstanten Wert von etwa 0,0715, während für $b$ ebenfalls eine Abhängigkeit von der Grashof-Zahl $G r_{\mathrm{x} / \mathrm{L}, \mathrm{m}}$ auftritt (Gleichung 47).

$$
b=\left\{\begin{array}{cc}
0,0178 \cdot \ln \left(G r_{\mathrm{x} / L, \mathrm{~m}}\right)-0,2570 & \text { für } G r_{\mathrm{x} / \mathrm{L}, \mathrm{m}} \leq 10^{9} \\
-0,0102 \cdot \ln \left(G r_{\mathrm{x} / L, \mathrm{~m}}\right)+0,3289 & \text { für } G r_{\mathrm{x} / L, \mathrm{~m}}>10^{9}
\end{array}\right.
$$




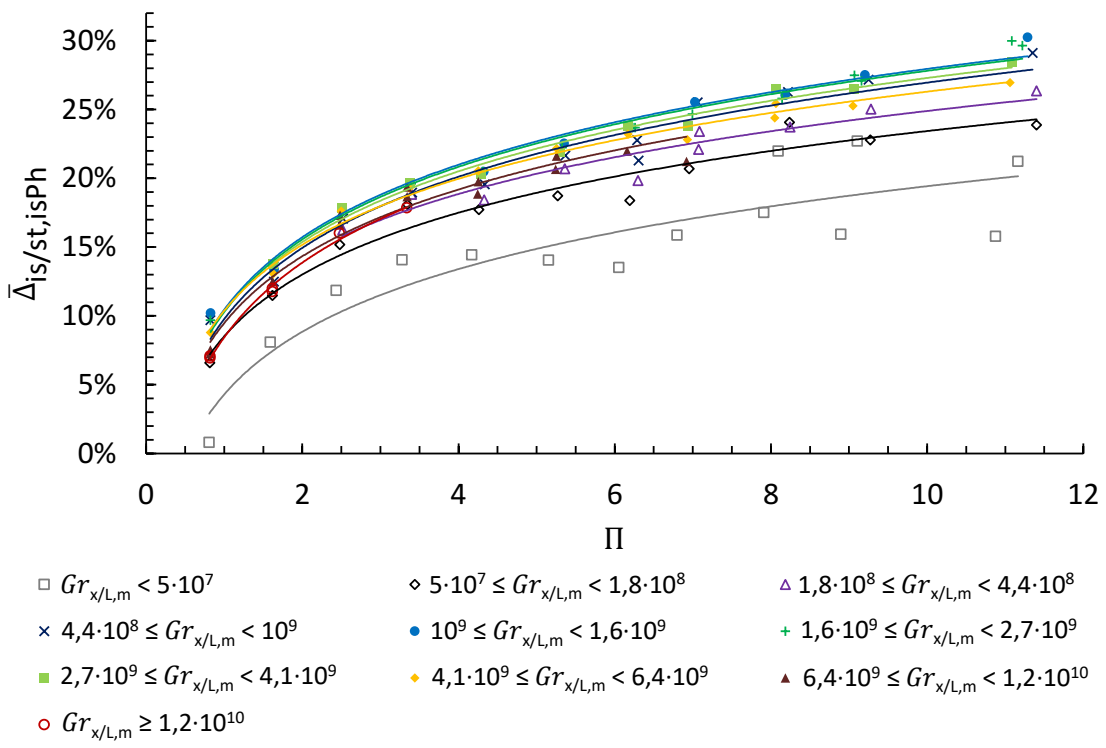

Abbildung 5.13: Zusätzliche konvektive Wärmeübertragung als Funktion von $\Pi$

Zu den Validierungsmessungen mit sprungartiger Änderung der Randbedingungen und anschließender Beharrung ist eine ausführliche Ergebnisübersicht im Anhang A.2 aufgeführt.

\subsubsection{Instationäre Phase bei sprungartigen Änderungen}

Die Dauer der instationären Phase $\Delta t_{\text {isPh, }}$ in der die konvektive Wärmeübertragung gemäß Gleichung 31 um mehr als ein Prozent vom quasi-stationären Vergleichsniveau der Randbedingungen eines Zeitpunktes abweicht, kann für sprungartige Änderungen im Verhältnis zur jeweiligen Sprungdauer $\Delta t_{\text {Sprung }}$ gemäß Gleichung 48 bestimmt werden.

$$
\frac{\Delta t_{\text {isph }}}{\Delta t_{\text {sprung }}}=98,0279 \cdot\left(\frac{\dot{q}_{\max }}{\Delta \vartheta_{\text {Sprung }} \cdot C_{\mathrm{W}} / A_{\mathrm{W}}}\right)^{0,7624}
$$

Der verwendete Regressionsparameter berücksichtigt die maximale ImpulsWärmestromdichte $\dot{q}_{\text {max }}$, die Temperaturdifferenz des Sprunges $\Delta \vartheta_{\text {Sprung }}$ und die flächenbezogene Wärmekapazität der vertikalen Platte $C_{\mathrm{W}} / A_{\mathrm{W}}$. Er weist in Analogie zum Regressionsparameter der instationären Phase für zyklische 
Randbedingungs-Variationen (vgl. Gleichung 42) die Einheit 1/s auf und repräsentiert im physikalischen Sinne die (reziproke) Dauer des Temperatursprunges $1 / \Delta t_{\text {Sprung. }}$. Die dabei getroffene Vereinfachung einer Vernachlässigung der wärmeabgebenden Terme der Gleichung 29 ist nur dann zulässig, wenn die zugeführte elektrische Leistung $\dot{q}_{\mathrm{el}}$ vergleichsweise groß ist. So beträgt die mittlere elektrische Leistungszufuhr $\dot{\dot{q}}_{\text {el }}$ während des Temperatursprunges $\Delta t_{\text {Sprung }}$ in den durchgeführten Validierungsmessungen stets mindestens das Doppelte der mittleren Wärmeabgabe aufgrund von Konvektion $\overline{\dot{q}}_{\mathrm{c}}$ und Strahlung $\overline{\dot{q}}_{\mathrm{r}}$. Kann diese Voraussetzung nicht erfüllt werden, ist eine iterative Vorgehensweise erforderlich, die die tatsächliche (instationäre) Wärmeabgabe $\dot{q}_{c, \text { is }}$ bei der Ermittlung der Dauer des Temperatursprunges $\Delta t_{\text {Sprung }}$ berücksichtigt.

Für die in Abbildung 5.14 dargestellten Regressionsdaten der Validierungsmessungen beläuft sich die Dauer der instationären Phase $\Delta t_{\text {isPh }}$ in etwa auf das 0,7 bis 8,0-fache der Sprungdauer $\Delta t_{\text {Sprung; }}$; das arithmetische Mittel beträgt $\Delta t_{\text {isPh }} / \Delta t_{\text {Sprung }}=4,25$.

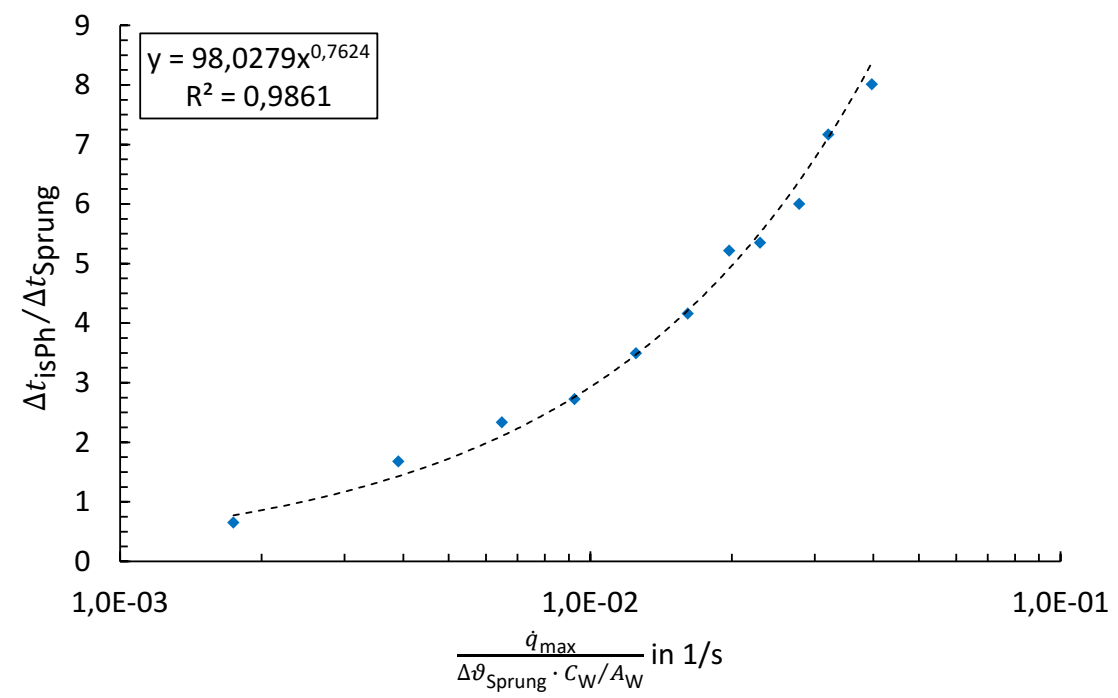

Abbildung 5.14: Dauer der instationären Phase bei sprungartigen Änderungen 
Eine Abschätzung der dimensionsbehafteten Dauer des Temperatursprunges $\Delta t_{\text {Sprung }}$ gelingt unter den genannten Vereinfachungen mit Hilfe desselben Regressionsparameters nach Gleichung 49.

$$
\Delta t_{\text {Sprung }}=0,6617 \cdot\left(\frac{\dot{q}_{\max }}{\Delta \vartheta_{\text {sprung }} \cdot C_{\mathrm{W}} / A_{\mathrm{W}}}\right)^{-1,1492}
$$

Gemäß der physikalischen Modellvorstellung aus Kapitel 5.1.1 wird die Sprungdauer in Analogie zur Impulsdauer (vgl. Kapitel 5.2.2) als der Zeitraum interpretiert, in dem ein Überschuss an potentieller Energie im Fluid aufgebaut wird. Die Angleichung von $e_{\text {pot }} / e_{\text {kin }}$ an das stationäre Verhältnis erfolgt wiederum nach einer (starken) Reduzierung der Leistungszufuhr. Dementsprechend liegt auch für die Versuchsreihen mit sprungartiger Änderung der Randbedingungen keine direkte Abhängigkeit der Dauer der instationären Phase von den Eigenschaften der wärmeübertragenden Oberfläche vor.

Ferner zeigt sich für die sprungartigen Änderungen in Übereinstimmung mit Kapitel 5.2.2 kein Zusammenhang zwischen der Dauer der instationären Phase und der mittleren Grashof-Zahl. Voraussetzung hierfür ist ebenfalls, dass die Leistungszufuhr während des Temperatursprunges hinreichend groß ist (d.h. im Vergleich zur Wärmeabgabe dominiert), um an jeder Stelle der beheizten Oberfläche die gleiche Sprungdauer zu ermöglichen. Als mögliche Einschränkung der Regressionsfunktionen der Gleichungen 48 und 49 auf die individuellen Gegebenheiten des konkreten Versuchsaufbaus verbleibt daher auch für die sprungartigen Änderungen primär die Prandtl-Zahl.

\subsubsection{Momentanwerte von sprungartigen Änderungen}

Zur Prognose des zeitlichen Verlaufs der instationären konvektiven Wärmeübertragung bei sprungartigen Änderungen der Strömungs-Randbedingungen wird ebenfalls die bereits in Kapitel 5.2.3 für zyklische Variationen beschriebene Methodik vorgeschlagen. Dabei ist die Impulsdauer $\Delta t_{\text {Impuls }}$ jedoch gemäß Gleichung 50 durch die Dauer des Temperatur-Sprunges $\Delta t_{\text {sprung }}$ zu ersetzen.

$$
\Delta_{\text {is } / \mathrm{st}}=\left\{\begin{array}{cl}
\frac{2 \cdot \bar{\Delta}_{\text {is/st,isph }}}{\Delta t_{\text {Sprung }}} \cdot t & \text { für } t \leq \Delta t_{\text {Sprung }} \\
2 \cdot \bar{\Delta}_{\mathrm{is} / \mathrm{st}, \mathrm{isPh}}-\left[\frac{2 \cdot \bar{\Delta}_{\mathrm{is} / \mathrm{st} \text {,ish }}}{\Delta t_{\text {isph }}-\Delta t_{\text {Sprung }}} \cdot\left(t-\Delta t_{\text {Sprung }}\right)\right] & \text { für } t>\Delta t_{\text {Sprung }}
\end{array}\right. \text { (GI. 50) }
$$


Den Beobachtungen aus den Kapiteln 3.4.2 und 4.1.2 entsprechend, wird das Maximum der Momentanwerte $\Delta_{\text {is/st }}$ dabei zum Ende eines TemperaturSprunges $\Delta t_{\text {Sprung }}$ erwartet, da zu diesem Zeitpunkt eine starke Reduzierung der Wärmestromzufuhr $\dot{q}_{\text {Impuls }}$ erfolgt.

Eine Prognose der Momentanwerte der insgesamt vorliegenden instationären konvektiven Wärmeübertragungs-Intensität $\dot{q}_{\mathrm{c}, \text { is }}$ gelingt in Übereinstimmung mit Kapitel 5.2.3 gemäß Gleichung 44. Abbildung 5.15 zeigt die resultierende Ergänzung der Versuchsreihe aus Abbildung 3.26 um die prognostizierten Momentanwerte der konvektiven Wärmeübertragung $\dot{q}_{c, \text { is }}$ gemäß Gleichung 44 .

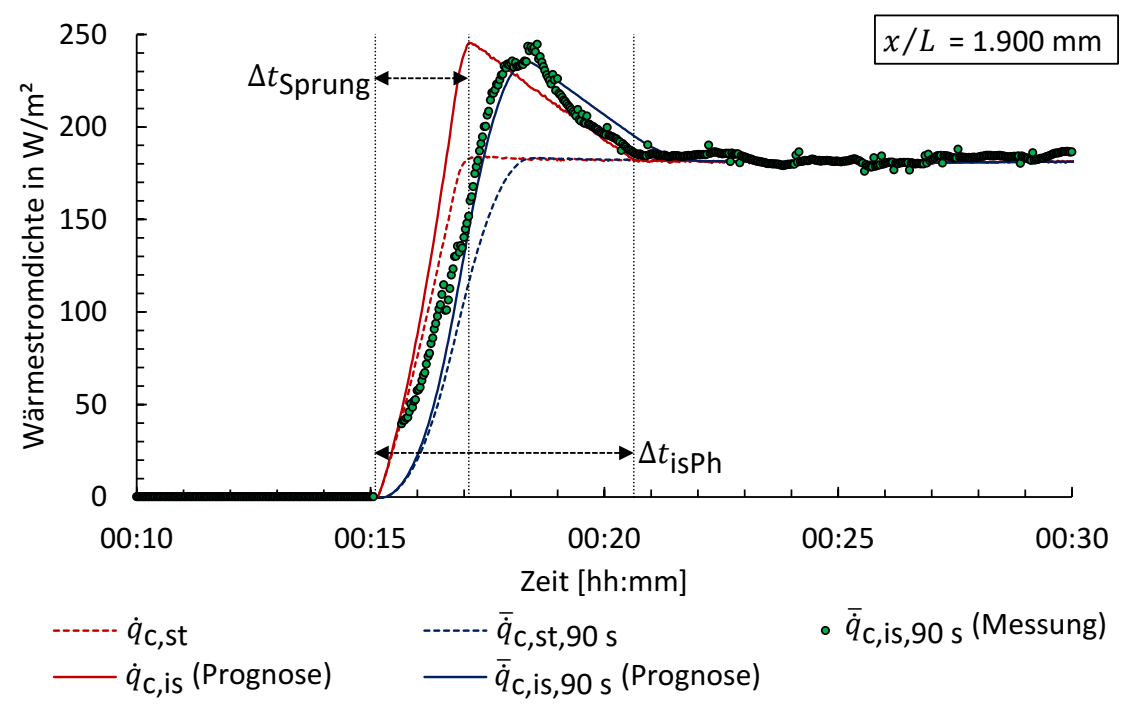

Abbildung 5.15: Momentanwert-Prognose für sprungartige Änderungen (mit $\dot{q}_{\text {max }}=1.429 \mathrm{~W} / \mathrm{m}^{2}$ und $\Delta \vartheta_{\text {Sprung }}=40 \mathrm{~K}$ )

Wie im Falle der Versuchsreihen mit zyklischen Variationen ist für den dargestellten Beispielfall sowie für alle betrachteten Versuchsreihen zu sprungartigen Randbedingungs-Änderungen eine gute grafische Übereinstimmung der prognostizierten Momentanwerte $\overline{\dot{q}}_{c, i s, 90 \text { s }}$ mit den jeweils gemessenen Werten $\overline{\dot{q}}_{\mathrm{c}, \mathrm{is}, 90 \mathrm{~s}}$ festzustellen. Dabei gilt die in Kapitel 5.2.3 diskutierte eingeschränkte Vergleichbarkeit von Prognose- und Messwerten für Impulse mit $\Delta t_{\text {Impuls }} \leq 60 \mathrm{~s}$ analog für Sprung-Dauern $\Delta t_{\text {Sprung }} \leq 60 \mathrm{~s}$. 
In Gleichung 50 bleibt hingegen unberücksichtigt, dass der konvektive Wärmeübergang $\alpha_{\mathrm{c}}$ im Falle einer anfangs vollständig ausgekühlten Plattenoberfläche $\left(\vartheta_{W}=\vartheta_{\infty}\right)$ gemäß Gleichung 2 unmittelbar nach dem Beginn einer Wärmestromzufuhr (sobald $\vartheta_{\mathrm{W}}>\vartheta_{\infty}$ ) unendlich groß bilanziert wird. Dieser physikalisch unbegründete Effekt, der dem Konzept des Bezugs von Wärmestromdichten auf Temperaturdifferenzen im stationären Fall geschuldet ist, wird durch das verwendete Mittelungsintervall von $90 \mathrm{~s}$ jedoch ohnehin egalisiert.

\subsubsection{Modellanwendung und Prognosegüte für sprungartige Änderungen}

Abbildung 5.16 zeigt schematisch die Vorgehensweise des vorgestellten Prognosemodells zur instationären konvektiven Wärmeübertragung bei sprungartigen Änderungen der Randbedingungen mit anschließender Beharrung.

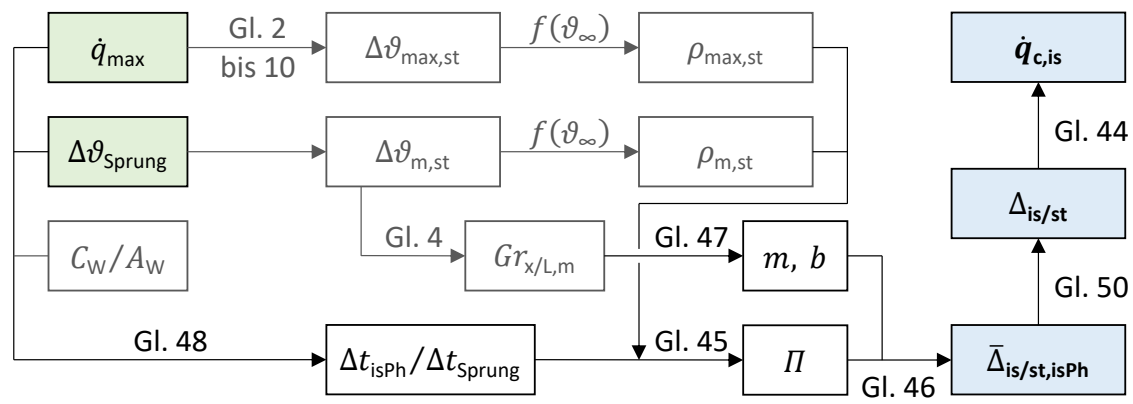

Abbildung 5.16: Anwendungsschema des Regressionsmodells für sprungartige Änderungen

Als Eingangsgrößen dienen dabei die maximale Wärmestromdichte $\dot{q}_{\max }$, die Temperaturdifferenz des Sprunges $\Delta \vartheta_{\text {Sprung }}$ sowie die flächenspezifische Wärmekapazität der Plattenoberfläche $C_{\mathrm{W}} / A_{\mathrm{W}}$. In Übereinstimmung mit Kapitel 5.2.4 werden zunächst die maximalen und mittleren Temperaturdifferenzen $\Delta \vartheta_{\text {max,st }}$ und $\Delta \vartheta_{\text {m,st }}$ des stationären Vergleichsfalls mit bekannten BerechnungsKorrelationen ermittelt, aus denen anschließend die zugehörigen Fluiddichten $\rho_{\text {max }, \text { st }}$ und $\rho_{\mathrm{m}, \mathrm{st}}$ resultieren. Unter Hinzunahme der Dauer der instationären Phase im Verhältnis zur Dauer des Sprunges $\Delta t_{\text {isph }} / \Delta t_{\text {sprung }}$ kann die dimensi- 
onslose Größe $\Pi$ berechnet werden, die zusammen mit den Regressionsparametern $m$ und $b$ zur mittleren Abweichung zwischen der instationären konvektiven Wärmeübertragung und dem quasi-stationären Vergleichsfall $\bar{\Delta}_{\text {is/st,isph }}$ führt. Unter der Annahme einer kontinuierlichen, linearen zeitlichen Zuwachsund Abklingrate der konvektiven Wärmeübertragungs-Intensität können die zugehörigen Momentanwerte $\Delta_{\text {is/st }}$ bestimmt werden. Wie im Falle der zyklisch variierten Randbedingungen werden für die Prognose der insgesamt vorhandenen instationären konvektiven Wärmestromdichte eines Zeitpunkts $\dot{q}_{c, \text { is }}$ schließlich die quasi-stationären Vergleichswerte addiert.

Ein Vergleich zwischen den Validierungsmessungen für sprungartige Änderungen und den zugehörigen Prognosewerten des Berechnungsmodells der Gleichungen 45 bis 50 ist in Abbildung 5.17 dargestellt.

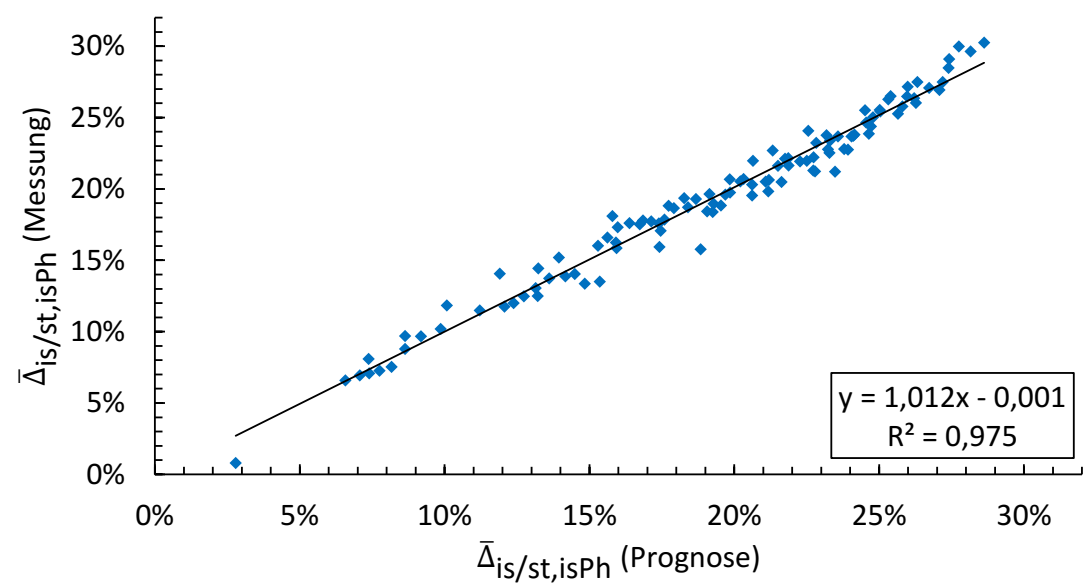

Abbildung 5.17: Prognosegüte des Regressionsmodells für sprungartige Änderungen

Dabei wird insgesamt ebenfalls eine gute Übereinstimmung deutlich. Die absoluten Differenzen zwischen Prognose und Messung liegen für die 110 Einzelergebnisse zwischen $0 \%$ im Minimum und 3,1\% im Maximum, wobei das arithmetische Mittel 0,8\% beträgt. Die größten Prognosefehler treten in Übereinstimmung mit der Standardabweichung der Wiederholungsmessungen (vgl. Kapitel 5.3.1) wiederum für kleine Grashof-Zahlen $\left(G r_{x / L, m}<10^{8}\right)$ auf. 


\section{Zusammenfassung}

Im Rahmen der dargestellten Untersuchungen wird die konvektive Wärmeübertragung durch instationäre, natürliche Auftriebsströmungen an der vertikalen Platte betrachtet. Für derartige Strömungsformen ist aufgrund einer Kopplung von Energie- und Impulstransport bislang keine Möglichkeit zur analytischen Berechnung des Temperatur- und Geschwindigkeitsfeldes bekannt. Im Falle von vollständig ausgebildeten Strömungen werden daher semi-empirische Näherungs-Korrelationen zur Berechnung der Wärmeübertragungs-Intensität herangezogen.

Bisherige Betrachtungen von zeitlich veränderlichen freien Konvektionsströmungen deuten bei hinreichend langsamen Änderungen auf ein quasistationäres Verhalten der Wärmeübertragung hin. Für schnelle Änderungen werden hingegen irreguläre Schwankungen der Grenzschichtgrößen beschrieben (vgl. Kapitel 2.1). Der Fokus dieser Ausarbeitung liegt daher auf einer Quantifizierung der Auswirkungen derartiger instationärer Strömungsfluktuationen auf die konvektive Wärmeübertragung.

Mit Ausnahme von direkten Ansätzen lassen numerische Simulationen bei einer Analyse der beschriebenen Strömungsart aufgrund von verschiedenen Einschränkungen der zugrundeliegenden Modellannahmen größere Unsicherheiten erwarten (vgl. Kapitel 2.2). Die durchgeführten Betrachtungen beruhen daher auf einer experimentellen Herangehensweise. Dazu wird die Intensität der konvektiven Wärmeübertragung an einer direkt-elektrisch beheizten Kupferplatte messtechnisch erfasst (vgl. Kapitel 3.1). Eine symmetrische Wärmeabgabe und eine geringe thermische Eigenkapazität der beheizten Platte ermöglichen dabei auch die Messung von Momentanwerten der WärmeübertragungsIntensität im instationären Fall. Der insgesamt $2 \mathrm{~m}$ hohen und $1 \mathrm{~m}$ breiten Platte werden Impuls-Wärmestromdichten von bis zu $1.430 \mathrm{~W} / \mathrm{m}^{2}$ aufgeprägt, was kurzfristigen Grashof-Zahlen von etwa $G r_{\mathrm{L}}^{*} \approx 4,2 \cdot 10^{13}$ entspricht. Oberflächentemperaturen von maximal $100^{\circ} \mathrm{C}$ im Dauerbetrieb führen im Maximum zu stationären Grashof-Zahlen von $G r_{\mathrm{L}} \approx 5,8 \cdot 10^{10}$. Betrachtet werden dementsprechend sämtliche Strömungsformen (laminar, transient und vollturbulent). Eine Validierung der stationären Wärmeübertragungs-Intensität zeigt eine sehr gute Übereinstimmung zu bisherigen Literaturdaten. 
Die instationäre Bewertung erfolgt im Vergleich zu einer quasi-stationären Betrachtungsweise, bei der der Strömung zu jedem Zeitpunkt die Wärmeübertragungs-Intensität unterstellt wird, die sich für die jeweils aktuellen Randbedingungen im stationären Fall einstellen würde (vgl. Kapitel 3.3).

Für eine grundlegende Analyse von Kausalzusammenhängen zwischen der konvektiven Wärmeübertragung und variablen Strömungs-Randbedingungen einer zyklischen Betriebsweise werden zunächst 52 Versuchsreihen mit Wärmestromdichten zwischen $57 \mathrm{~W} / \mathrm{m}^{2}$ und $1.430 \mathrm{~W} / \mathrm{m}^{2}$ bei Impulszeiten von $15 \mathrm{~s}$ bis $1.800 \mathrm{~s}$ und einer Gesamt-Zyklusdauer von $510 \mathrm{~s}$ bis $2.700 \mathrm{~s}$ durchgeführt. Der Verlauf der Momentanwerte der konvektiven Wärmeabfuhr zeigt dabei während eines plötzlichen Wärmestrom-Impulses sowie einige Zeit danach eine deutliche Überschreitung der quasi-stationären Vergleichswerte. Im zeitlichen Mittel dieser instationären Phase wird eine Intensivierung der konvektiven Wärmeübertragung von bis zu 23,8 \% festgestellt. Nach dem Ende eines Wärmestromimpulses zeigt sich zunächst eine asymptotische Annäherung der Momentanwerte an das quasi-stationäre Vergleichsniveau. Der Auskühlvorgang erfolgt schließlich in sämtlichen Versuchsreihen aufgrund der verhältnismäßig langsamen zeitlichen Änderung der Oberflächentemperatur mit guter Näherung quasi-stationär.

Für sprungartige Änderungen der Strömungs-Randbedingungen mit anschließender Beharrung zeigt sich grundsätzlich eine sehr ähnliche Beeinflussung der Wärmeübertragungs-Intensität. Aufgrund der Beharrung der Wärmezufuhr verläuft die asymptotische Annäherung der Momentanwerte an das quasistationäre Vergleichsniveau jedoch langsamer, als bei der plötzlichen Beendigung der Beheizung im Falle der zyklischen Variationen. In 29 Versuchsreihen mit maximalen Wärmestromdichten zwischen $74 \mathrm{~W} / \mathrm{m}^{2}$ und $1.430 \mathrm{~W} / \mathrm{m}^{2}$ und Beharrungsniveaus der Oberflächentemperatur von $30^{\circ} \mathrm{C}$ bis $90{ }^{\circ} \mathrm{C}$ bei einer Umgebungstemperatur von $20^{\circ} \mathrm{C}$ zeigen sich für die sprungartigen Änderungen mittlere Abweichungen der Wärmeübertragungs-Intensität gegenüber dem quasi-stationären Vergleichsniveau von bis zu 30,2 \% innerhalb der instationären Phase.

Für beide Arten der Randbedingungsvariation liegt eine ausgeprägte Abhängigkeit der Intensivierung der konvektiven Wärmeübertragung von der mittleren Grashof-Zahl vor. So treten die Maximalwerte der instationären Wärmeüber- 
tragung stets zu Beginn des laminar-turbulenten Transitionsgebiets auf, da die Strömung dort am anfälligsten für Stabilitätsstörungen ist und ein lokales Minimum in der Wärmeübertragungsintensität aufweist.

Die Beeinflussung der freien Konvektionsströmung durch eine instationäre Betriebsweise wird darüber hinaus phänomenologisch mit Hilfe von Strömungsvisualisierungen und Messungen der Geschwindigkeitsfluktuationen untersucht (vgl. Kapitel 4). Auch dabei zeigen sich nach einer impulsartigen Variation der Wärmestromzufuhr der beheizten Platte temporär deutliche Veränderungen der Strömungsstrukturen. So bewirkt eine Beschleunigung von wandnahem Fluid im Übergangsbereich zu ruhendem Umgebungsfluid Scherbewegungen, die sich zu großskaligen Rotationsstrukturen (insbesondere Kelvin-HelmholtzWirbeln) fortpflanzen und so eine verstärkte Durchmischung der Strömungsgrenzschicht verursachen. Im weiteren zeitlichen Verlauf bilden sich die instationären Strukturen wieder vollständig zurück, sodass eine quasi-stationäre Strömung verbleibt.

Die exemplarische Messung von Strömungsgeschwindigkeiten innerhalb der Grenzschicht lässt während eines Wärmestrom-Impulses sowie einige Zeit danach ebenfalls stark ausgeprägte, irreguläre Fluktuationen erkennen. Diese stehen sowohl in guter Übereinstimmung mit den bisherigen Literaturdaten, als auch mit der gemessenen Dauer der instationären Phase der konvektiven Wärmeübertragung.

Die experimentellen Erkenntnisse legen die physikalische Modellvorstellung nahe, dass nach einer plötzlichen Erhöhung der Wärmeübertragung von der Wand an das Fluid ein Überschuss an potentieller Energie in der Auftriebsströmung vorliegt, der durch das vorübergehende Aufkommen von instationären Strömungsstrukturen abgebaut wird, bis sich wieder ein stationäres Verhältnis zwischen potentieller und kinetischer Energie einstellt.

Zur Prognose des Einflusses der instationären Strömungsstrukturen auf die mittlere konvektive Wärmeübertragung wird eine dimensionslose Kennzahl $\Pi$ vorgestellt (vgl. Kapitel 5), die gemäß der physikalischen Modellvorstellung den mittleren Überschusses der potentiellen Energie innerhalb der instationären Phase ausdrückt. Für das Berechnungsmodell, das außerdem die jeweilige mittlere Grashof-Zahl berücksichtigt, werden die erforderlichen Regressionsparameter anhand von fünffach wiederholten Versuchsreihen bestimmt. Die erar- 
beiteten Prognosemodelle weisen gegenüber den Messwerten mittlere Gesamtfehler von ca. 1,1\% bei zyklisch variierten Randbedingungen und von etwa $0,9 \%$ bei sprungartigen Änderungen auf.

Neben der Prognose von mittleren Werten wird auch ein Modell zur Berechnung von Momentanwerten der instationären konvektiven Wärmeübertragung vorgestellt. Dies beruht auf der Annahme, dass die zeitliche Änderung der Wärmeübertragungs-Intensität bei konstanter Beschleunigung im Gravitationsfeld in erster Näherung linear verläuft. Der so prognostizierte zeitliche Verlauf der instationären Wärmeübertragungs-Intensität zeigt eine gute grafische Übereinstimmung mit gemessenen Momentanwerten. 


\section{Ausblick}

Die dargestellten Erkenntnisse zur Wärmeübertragung durch instationäre freie Konvektion an der vertikalen Platte beruhen ausschließlich auf Untersuchungen, die in Luft durchgeführt werden. Dementsprechend stellt sich für weiterführende Betrachtungen zunächst die grundsätzliche Frage, ob die vorgestellten analytischen Berechnungsmethoden in abweichenden Fluiden ihre Gültigkeit behalten.

Damit einhergehend erscheint insbesondere eine Betrachtung zur Abhängigkeit der Dauer der instationären Phase der konvektiven Wärmeübertragung von der Prandtl-Zahl sinnvoll. So zeigt sich im Rahmen der hier durchgeführten Versuchsreihen eine ausschließliche Abhängigkeit der Dauer der instationären Phase von der Dauer eines Wärmestrom-Impulses und von der Gesamtdauer eines Zyklus oder Sprungs (vgl. Kapitel 5.2.2 und 5.3.2). Aufgrund des individuellen Verhältnisses von Impuls- und Wärmetransport innerhalb eines Fluids liegt jedoch auch eine individuelle Dauer der Umwandlung von plötzlich eingebrachter potentieller Energie in kinetische Energie durch instationäre Strömungsstrukturen nahe.

Weitere Fragestellungen resultieren zudem bezüglich des Vorkommens und der Intensität einer instationären konvektiven Wärmeübertragung bei abweichenden Strömungs-Geometrien und Strömungs-Arten (z.B. Mischkonvektion).

Zur Entwicklung von Modellen für die numerische Simulation von instationären freien Konvektionsströmungen ist ferner eine Untersuchung der zeitlichen Entwicklung der Geschwindigkeiten im Strömungsfeld erforderlich, da im Rahmen dieser Untersuchung aufgrund einer invasiven Messmethodik lediglich eine phänomenologische Analyse der Fluktuationen erfolgt (vgl. Kapitel 4.2).

Für die vertikale Platte erscheint darüber hinaus eine Betrachtung von weiteren Randbedingungs-Variationen sinnvoll. Beispielsweise wäre die gezielt instationäre Betriebsweise einer Raumheizfläche mit wasserbasierter Wärmezufuhr vergleichbar mit der impulsartigen Aufprägung einer inhomogenen Wärmestromdichte. Die verzögerte asymptotische Annäherung der Momentanwerte der instationären konvektiven Wärmeübertragung an das quasi-stationäre Vergleichsniveau bei sprungartigen Änderungen mit anschließender Beharrung im Vergleich zur vollständigen Beendigung der Wärmestromzufuhr bei zykli- 
schen Variationen (vgl. Kapitel 3.4.2) legt außerdem eine Betrachtung weiterer Signalformen nahe. Beispielhaft sei hier eine Kombination aus zyklischen Variationen und einer zeitlich begrenzten Beharrung genannt. 


\section{A Anhang}

Nachfolgend werden die Ergebnisse der Validierungs-Versuchsreihen zur instationären Betriebsweise in tabellarischer Form aufgeführt. Angegeben ist jeweils das arithmetische Mittel aus fünf Wiederholungsmessungen (vgl. Kapitel 5.2.1 und 5.3.1). Gemäß der in Kapitel 1.1.1 beschriebenen Konvention zur Auswertung von Teilbereichen des Strömungsgebiets liegen für jede Versuchsreihe zehn Bilanzergebnisse vor. Die Zeilen der aufgelisteten Versuchsreihen beziehen sich somit jeweils auf die Strömungs-Teilbereiche $x / L=100 \mathrm{~mm}$, $x / L=300 \mathrm{~mm}, x / L=500 \mathrm{~mm}, x / L=700 \mathrm{~mm}, x / L=900 \mathrm{~mm}, x / L=1.100 \mathrm{~mm}$, $x / L=1.300 \mathrm{~mm}, x / L=1.500 \mathrm{~mm}, x / L=1.700 \mathrm{~mm}$ und $x / L=1.900 \mathrm{~mm}$.

\section{A.1 Ergebnisse der Validierungsmessungen für zyklische Variationen}

Tabelle A.1 enthält die Betrachtungsergebnisse der Validierungs-Versuchsreihen für zyklisch variierte Randbedingungen (Kapitel 5.2.1). Die Randbedingungen werden im Format hh:mm:ss sowie in den Einheiten $\mathrm{W} / \mathrm{m}^{2}, \mathrm{~K}$ und $\mathrm{K} / \mathrm{s}$ angegeben.

Tabelle A.1: Ergebnisübersicht der Validierungsmessungen für zyklische Variationen

\begin{tabular}{|c|c|c|c|c|c|c|c|}
\hline \multicolumn{2}{|c|}{ Randbedingungen } & $\overline{G r}_{\mathrm{x} / \mathrm{L}, \mathrm{Zykl} .}$ & $\overline{\boldsymbol{G}}_{\mathrm{x} / \mathrm{L}, \mathrm{Zykl} .}^{*}$ & $\Pi^{*}$ & $\overline{N u}_{\text {st, }, \mathrm{L} / \mathrm{Z}, \mathrm{yk} \mathrm{l}}$ & \multicolumn{2}{|c|}{$\bar{\Delta}_{\text {is } / \text { st,isPh }} \stackrel{S}{\text { (5 Wdh.) }}$} \\
\hline \multicolumn{2}{|c|}{$\Delta t_{\text {Impuls }}=180 \mathrm{~s}, \Delta t_{\text {Auskühlung }}=300 \mathrm{~s}$} & $4,44 \mathrm{E}+07$ & $1,90 \mathrm{E}+09$ & 1,99 & 45 & $-1,7 \%$ & $\pm 0,5 \%$ \\
\hline$\Delta t_{\text {Zyklus }}$ & 00:07:58 & $3,74 \mathrm{E}+08$ & $2,94 \mathrm{E}+10$ & 2,01 & 76 & $5,7 \%$ & $\pm 0,3 \%$ \\
\hline$\Delta t_{\text {isPh }}$ & 00:04:12 & $1,31 E+09$ & $1,46 \mathrm{E}+11$ & 2,03 & 105 & $8,7 \%$ & $\pm 0,2 \%$ \\
\hline$\overline{\dot{q}}_{\text {Zyklus }}$ & 316 & $3,17 E+09$ & $4,54 \mathrm{E}+11$ & 2,03 & 135 & $9,2 \%$ & $\pm 0,3 \%$ \\
\hline$\overline{\dot{q}}_{\text {isPh }}$ & 358 & $6,24 \mathrm{E}+09$ & $1,10 \mathrm{E}+12$ & 2,02 & 170 & $6,9 \%$ & $\pm 0,3 \%$ \\
\hline$\dot{q}_{\text {Impuls }}$ & 843,1 & $1,07 \mathrm{E}+10$ & $2,30 E+12$ & 2,02 & 212 & $4,4 \%$ & $\pm 0,5 \%$ \\
\hline$\Delta \vartheta_{\mathrm{L}, \min }$ & 49,0 & $1,69 \mathrm{E}+10$ & $4,29 E+12$ & 2,01 & 254 & $3,2 \%$ & $\pm 0,5 \%$ \\
\hline$\Delta \vartheta_{L, \max }$ & 73,1 & $2,49 E+10$ & $7,36 \mathrm{E}+12$ & 2,00 & 294 & $3,4 \%$ & $\pm 0,4 \%$ \\
\hline$d \vartheta_{\mathrm{W}, \mathrm{L}} / d t_{1 \tau, \mathrm{Impuls}}$ & 0,145 & $3,53 E+10$ & $1,18 \mathrm{E}+13$ & 2,00 & 334 & $3,6 \%$ & $\pm 0,4 \%$ \\
\hline$d \vartheta_{\mathrm{W}, \mathrm{L}} / d t_{1 \tau, \text { Auskühlung }}$ & $-0,091$ & $4,81 E+10$ & $1,81 \mathrm{E}+13$ & 2,00 & 374 & $3,7 \%$ & $\pm 0,3 \%$ \\
\hline
\end{tabular}


Tabelle A.1 (Fortsetzung): Ergebnisübersicht der Validierungsmessungen für zyklische Variationen

\begin{tabular}{|c|c|c|c|c|c|c|c|}
\hline \multicolumn{2}{|c|}{ Randbedingungen } & $\overline{\boldsymbol{G r}}_{\mathrm{x} / \mathrm{L}, \mathrm{zykl}}$ & $\overline{\boldsymbol{G}}_{\mathrm{x} / \mathrm{L}, \mathrm{Z} \mathrm{ykl} .}^{*}$ & $\Pi^{*}$ & $\overline{N u}_{\text {st }, \mathrm{x} / \mathrm{L}, \mathrm{zykl} .}$ & \multicolumn{2}{|c|}{$\bar{\Delta}_{\mathrm{is} / \mathrm{st}, \mathrm{isPh}} \stackrel{S}{\text { (5 Wdh.) }}$} \\
\hline \multicolumn{2}{|c|}{$\bar{c} \Delta t_{\text {Impuls }}=292 \mathrm{~s}, \Delta t_{\text {Auskühlung }}=589 \mathrm{~s}$} & $1,42 \mathrm{E}+07$ & $4,67 E+08$ & 2,42 & 36 & $-3,5 \%$ & $\pm 0,9 \%$ \\
\hline$\Delta t_{\text {Zyklus }}$ & $00: 14: 40$ & $1,21 \mathrm{E}+08$ & $7,36 \mathrm{E}+09$ & 2,43 & 61 & $4,5 \%$ & $\pm 0,8 \%$ \\
\hline$\Delta t_{\text {isPh }}$ & 00:06:55 & $4,28 \mathrm{E}+08$ & $3,70 \mathrm{E}+10$ & 2,44 & 83 & $7,6 \%$ & $\pm 0,7 \%$ \\
\hline$\overline{\dot{q}}_{\text {Zyklus }}$ & 58 & $1,05 \mathrm{E}+09$ & $1,17 \mathrm{E}+11$ & 2,44 & 105 & $9,5 \%$ & $\pm 0,5 \%$ \\
\hline$\overline{\dot{q}}_{\text {isPh }}$ & 69 & $2,11 E+09$ & $2,84 \mathrm{E}+11$ & 2,44 & 128 & $9,6 \%$ & $\pm 0,4 \%$ \\
\hline$\dot{q}_{\text {Impuls }}$ & 175,6 & $3,69 E+09$ & $5,88 \mathrm{E}+11$ & 2,44 & 153 & $8,9 \%$ & $\pm 0,5 \%$ \\
\hline$\Delta \vartheta_{\mathrm{L}, \min }$ & 12,6 & $5,91 E+09$ & $1,09 \mathrm{E}+12$ & 2,44 & 179 & $7,4 \%$ & $\pm 0,6 \%$ \\
\hline$\Delta \vartheta_{L, \max }$ & 21,5 & $8,85 E+09$ & $1,86 \mathrm{E}+12$ & 2,44 & 205 & $7,3 \%$ & $\pm 0,8 \%$ \\
\hline$d \vartheta_{\mathrm{W}, \mathrm{L}} / d t_{1 \tau, \mathrm{Impuls}}$ & 0,033 & $1,26 \mathrm{E}+10$ & $2,98 \mathrm{E}+12$ & 2,44 & 234 & $6,3 \%$ & $\pm 1,1 \%$ \\
\hline$d \vartheta_{\mathrm{W}, \mathrm{L}} / d t_{1 \tau, \text { Auskühlung }}$ & $-0,018$ & $1,72 \mathrm{E}+10$ & $4,54 \mathrm{E}+12$ & 2,44 & 263 & $5,8 \%$ & $\pm 1,1 \%$ \\
\hline \multicolumn{2}{|c|}{$\Delta t_{\text {Impuls }}=87 \mathrm{~s}, \Delta t_{\text {Auskühlung }}=442 \mathrm{~s}$} & $2,41 \mathrm{E}+07$ & $8,93 E+08$ & 2,85 & 40 & $-1,1 \%$ & $\pm 0,9 \%$ \\
\hline$\Delta t_{\text {Zyklus }}$ & 00:08:48 & $2,04 \mathrm{E}+08$ & $1,40 \mathrm{E}+10$ & 2,89 & 67 & $7,1 \%$ & $\pm 0,3 \%$ \\
\hline$\Delta t_{\text {isPh }}$ & 00:03:41 & $7,21 E+08$ & $7,01 \mathrm{E}+10$ & 2,92 & 93 & $9,6 \%$ & $\pm 0,3 \%$ \\
\hline$\overline{\dot{q}}_{\text {Zyklus }}$ & 121 & $1,77 E+09$ & $2,20 E+11$ & 2,92 & 117 & $10,7 \%$ & $\pm 0,4 \%$ \\
\hline$\overline{\dot{q}}_{\text {isPh }}$ & 149 & $3,52 E+09$ & $5,35 E+11$ & 2,91 & 145 & $9,6 \%$ & $\pm 0,4 \%$ \\
\hline$\dot{q}_{\text {Impuls }}$ & 726,7 & $6,13 E+09$ & $1,11 \mathrm{E}+12$ & 2,90 & 175 & $7,8 \%$ & $\pm 0,7 \%$ \\
\hline$\Delta \vartheta_{L, \min }$ & 23,0 & $9,75 E+09$ & $2,05 \mathrm{E}+12$ & 2,89 & 207 & $6,1 \%$ & $\pm 0,7 \%$ \\
\hline$\Delta \vartheta_{L, \max }$ & 37,3 & $1,45 \mathrm{E}+10$ & $3,51 \mathrm{E}+12$ & 2,88 & 240 & $5,7 \%$ & $\pm 0,7 \%$ \\
\hline$d \vartheta_{\mathrm{W}, \mathrm{L}} / d t_{1 \tau, \mathrm{Impuls}}$ & 0,166 & $2,05 E+10$ & $5,63 \mathrm{E}+12$ & 2,88 & 275 & $5,2 \%$ & $\pm 0,7 \%$ \\
\hline$d \vartheta_{\mathrm{W}, \mathrm{L}} / d t_{1 \tau, \text { Auskühlung }}$ & $-0,038$ & $2,79 E+10$ & $8,60 \mathrm{E}+12$ & 2,87 & 309 & $4,9 \%$ & $\pm 0,6 \%$ \\
\hline \multicolumn{2}{|c|}{$\Delta t_{\text {Impuls }}=225 \mathrm{~s}, \Delta t_{\text {Auskühlung }}=968 \mathrm{~s}$} & $1,08 \mathrm{E}+07$ & $3,39 \mathrm{E}+08$ & 3,35 & 35 & $0,7 \%$ & $\pm 2,6 \%$ \\
\hline$\Delta t_{\text {zyklus }}$ & $00: 19: 52$ & $9,25 \mathrm{E}+07$ & $5,36 \mathrm{E}+09$ & 3,37 & 58 & $8,4 \%$ & $\pm 1,4 \%$ \\
\hline$\Delta t_{\text {isPh }}$ & 00:05:51 & $3,28 \mathrm{E}+08$ & $2,68 \mathrm{E}+10$ & 3,39 & 80 & $9,4 \%$ & $\pm 0,9 \%$ \\
\hline$\overline{\dot{q}}_{\text {Zyklus }}$ & 41 & $8,08 \mathrm{E}+08$ & $8,46 \mathrm{E}+10$ & 3,39 & 101 & $11,4 \%$ & $\pm 0,9 \%$ \\
\hline$\overline{\dot{q}}_{\text {isPh }}$ & 56 & $1,62 E+09$ & $2,06 \mathrm{E}+11$ & 3,39 & 122 & $11,8 \%$ & $\pm 0,6 \%$ \\
\hline$\dot{q}_{\text {Impuls }}$ & 217,3 & $2,85 E+09$ & $4,28 \mathrm{E}+11$ & 3,39 & 145 & $11,4 \%$ & $\pm 0,6 \%$ \\
\hline$\Delta \vartheta_{L, \min }$ & 8,3 & $4,57 E+09$ & $7,93 E+11$ & 3,39 & 170 & $10,3 \%$ & $\pm 0,8 \%$ \\
\hline$\Delta \vartheta_{L, \max }$ & 18,7 & $6,87 E+09$ & $1,35 \mathrm{E}+12$ & 3,38 & 192 & $10,4 \%$ & $\pm 0,7 \%$ \\
\hline$d \vartheta_{\mathrm{W}, \mathrm{L}} / d t_{1 \tau, \mathrm{Impuls}}$ & 0,049 & $9,77 E+09$ & $2,17 \mathrm{E}+12$ & 3,38 & 219 & $9,3 \%$ & $\pm 0,8 \%$ \\
\hline$d \vartheta_{\mathrm{W}, \mathrm{L}} / d t_{1 \tau, \text { Auskühlung }}$ & $-0,014$ & $1,34 \mathrm{E}+10$ & $3,31 \mathrm{E}+12$ & 3,38 & 247 & $8,3 \%$ & $\pm 0,7 \%$ \\
\hline \multicolumn{2}{|c|}{$\Delta t_{\text {Impuls }}=80 \mathrm{~s}, \Delta t_{\text {Auskühlung }}=660 \mathrm{~s}$} & $1,77 \mathrm{E}+07$ & $6,11 \mathrm{E}+08$ & 3,77 & 37 & $2,0 \%$ & $\pm 1,2 \%$ \\
\hline$\Delta t_{\text {zyklus }}$ & $00: 12: 18$ & $1,50 E+08$ & $9,61 \mathrm{E}+09$ & 3,83 & 63 & $9,2 \%$ & $\pm 0,5 \%$ \\
\hline$\Delta t_{\mathrm{isPh}}$ & 00:03:55 & $5,31 E+08$ & $4,83 E+10$ & 3,87 & 87 & $12,1 \%$ & $\pm 0,6 \%$ \\
\hline$\overline{\dot{q}}_{\text {Zyklus }}$ & 78 & $1,30 E+09$ & $1,52 \mathrm{E}+11$ & 3,87 & 111 & $13,1 \%$ & $\pm 0,5 \%$ \\
\hline$\overline{\dot{q}}_{\text {isPh }}$ & 108 & $2,61 E+09$ & $3,70 E+11$ & 3,86 & 135 & $12,8 \%$ & $\pm 0,3 \%$ \\
\hline$\dot{q}_{\text {Impuls }}$ & 724,5 & $4,57 E+09$ & $7,66 \mathrm{E}+11$ & 3,85 & 162 & $11,7 \%$ & $\pm 0,2 \%$ \\
\hline$\Delta \vartheta_{L, \min }$ & 15,3 & $7,29 E+09$ & $1,42 \mathrm{E}+12$ & 3,84 & 191 & $9,7 \%$ & $\pm 0,3 \%$ \\
\hline$\Delta \vartheta_{\mathrm{L}, \max }$ & 29,3 & $1,09 E+10$ & $2,42 E+12$ & 3,81 & 219 & $9,4 \%$ & $\pm 0,4 \%$ \\
\hline$d \vartheta_{\mathrm{W}, \mathrm{L}} / d t_{1 \tau, \mathrm{Impuls}}$ & 0,177 & $1,55 \mathrm{E}+10$ & $3,88 \mathrm{E}+12$ & 3,81 & 250 & $8,6 \%$ & $\pm 0,5 \%$ \\
\hline$d \vartheta_{\mathrm{W}, \mathrm{L}} / d t_{1 \tau, \text { Auskühlung }}$ & $-0,026$ & $2,10 E+10$ & $5,92 \mathrm{E}+12$ & 3,81 & 282 & $8,1 \%$ & $\pm 0,5 \%$ \\
\hline
\end{tabular}


Tabelle A.1 (Fortsetzung): Ergebnisübersicht der Validierungsmessungen für zyklische Variationen

\begin{tabular}{|c|c|c|c|c|c|c|c|}
\hline \multicolumn{2}{|c|}{ Randbedingungen } & $\overline{\boldsymbol{G r}}_{\mathrm{x} / \mathrm{L}, \mathrm{Zyk} \mathrm{l}}$ & $\overline{\boldsymbol{G r}}_{\mathrm{x} / \mathrm{L}, \mathrm{zykl}}^{*}$ & $\Pi^{*}$ & $\overline{N u}_{\text {st, } \mathrm{x} / \mathrm{L}, \mathrm{Zyk} \mathrm{l}}$ & \multicolumn{2}{|c|}{\begin{tabular}{cc}
$\bar{\Delta}_{\text {is } / \text { st, isPh }}$ & \multicolumn{1}{c}{ (5 Wdh.) }
\end{tabular}} \\
\hline \multicolumn{2}{|c|}{ 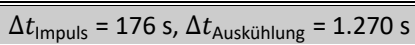 } & $9,21 E+06$ & $2,79 E+08$ & 4,27 & 34 & $1,2 \%$ & $\pm 3,0 \%$ \\
\hline$\Delta t_{\text {Zyklus }}$ & $00: 24: 04$ & $7,87 \mathrm{E}+07$ & $4,43 E+09$ & 4,30 & 57 & $9,6 \%$ & $\pm 2,3 \%$ \\
\hline$\Delta t_{\text {isPh }}$ & $00: 05: 28$ & $2,79 E+08$ & $2,23 E+10$ & 4,32 & 78 & $11,4 \%$ & $\pm 1,1 \%$ \\
\hline$\overline{\dot{q}}_{\text {Zyklus }}$ & 34 & $6,89 E+08$ & $7,00 \mathrm{E}+10$ & 4,33 & 98 & $12,8 \%$ & $\pm 0,5 \%$ \\
\hline$\overline{\dot{q}}_{\mathrm{isPh}}$ & 52 & $1,38 \mathrm{E}+09$ & $1,71 \mathrm{E}+11$ & 4,33 & 119 & $13,9 \%$ & $\pm 0,3 \%$ \\
\hline$\dot{q}_{\text {Impuls }}$ & 276,3 & $2,43 \mathrm{E}+09$ & $3,55 \mathrm{E}+11$ & 4,32 & 142 & $14,0 \%$ & $\pm 0,8 \%$ \\
\hline$\Delta \vartheta_{\mathrm{L}, \min }$ & 6,2 & $3,91 E+09$ & $6,58 \mathrm{E}+11$ & 4,32 & 165 & $13,0 \%$ & $\pm 0,7 \%$ \\
\hline$\Delta \vartheta_{L, \max }$ & 17,5 & $5,88 \mathrm{E}+09$ & $1,12 \mathrm{E}+12$ & 4,31 & 186 & $13,0 \%$ & $\pm 0,8 \%$ \\
\hline$d \vartheta_{\mathrm{W}, \mathrm{L}} / d t_{1 \tau, \mathrm{Impuls}}$ & 0,067 & $8,37 E+09$ & $1,79 \mathrm{E}+12$ & 4,31 & 211 & $11,5 \%$ & $\pm 0,9 \%$ \\
\hline$d \vartheta_{\mathrm{W}, \mathrm{L}} / d t_{1 \tau, \text { Auskühlung }}$ & $-0,012$ & $1,14 \mathrm{E}+10$ & $2,73 \mathrm{E}+12$ & 4,30 & 238 & $10,7 \%$ & $\pm 0,8 \%$ \\
\hline \multicolumn{2}{|c|}{$\Delta t_{\text {Impuls }}=73 \mathrm{~s}, \Delta t_{\text {Auskühlung }}=879 \mathrm{~s}$} & $1,37 \mathrm{E}+07$ & $4,51 \mathrm{E}+08$ & 4,70 & 36 & $1,9 \%$ & $\pm 1,4 \%$ \\
\hline$\Delta t_{\text {Zyklus }}$ & $00: 15: 52$ & $1,17 \mathrm{E}+08$ & $7,11 \mathrm{E}+09$ & 4,77 & 61 & $9,5 \%$ & $\pm 1,0 \%$ \\
\hline$\Delta t_{\text {isPh }}$ & 00:04:09 & $4,14 \mathrm{E}+08$ & $3,58 \mathrm{E}+10$ & 4,83 & 84 & $13,2 \%$ & $\pm 0,6 \%$ \\
\hline$\overline{\dot{q}}_{\text {zyklus }}$ & 56 & $1,02 E+09$ & $1,13 E+11$ & 4,83 & 106 & $14,7 \%$ & $\pm 0,3 \%$ \\
\hline$\overline{\dot{q}}_{\text {isPh }}$ & 85 & $2,04 E+09$ & $2,75 \mathrm{E}+11$ & 4,82 & 129 & $14,6 \%$ & $\pm 0,5 \%$ \\
\hline$\dot{q}_{\text {Impuls }}$ & 723,5 & $3,58 \mathrm{E}+09$ & $5,69 \mathrm{E}+11$ & 4,81 & 154 & $14,0 \%$ & $\pm 1,2 \%$ \\
\hline$\Delta \vartheta_{\mathrm{L}, \min }$ & 10,8 & $5,73 E+09$ & $1,05 \mathrm{E}+12$ & 4,79 & 180 & $12,8 \%$ & $\pm 1,1 \%$ \\
\hline$\Delta \vartheta_{\mathrm{L}, \max }$ & 24,3 & $8,58 \mathrm{E}+09$ & $1,80 \mathrm{E}+12$ & 4,76 & 205 & $12,6 \%$ & $\pm 1,5 \%$ \\
\hline$d \vartheta_{\mathrm{W}, \mathrm{L}} / d t_{1 \tau, \mathrm{Impuls}}$ & 0,182 & $1,22 \mathrm{E}+10$ & $2,88 \mathrm{E}+12$ & 4,76 & 235 & $11,5 \%$ & $\pm 1,6 \%$ \\
\hline$d \vartheta_{\mathrm{W}, \mathrm{L}} / d t_{1 \tau, \text { Auskühlung }}$ & $-0,020$ & $1,66 \mathrm{E}+10$ & $4,39 E+12$ & 4,76 & 265 & $10,8 \%$ & $\pm 1,6 \%$ \\
\hline \multicolumn{2}{|c|}{$\Delta t_{\text {Impuls }}=145 \mathrm{~s}, \Delta t_{\text {Auskühlung }}=1.540 \mathrm{~s}$} & $8,27 E+06$ & $2,47 E+08$ & 5,18 & 33 & $2,5 \%$ & $\pm 1,4 \%$ \\
\hline$\Delta t_{\text {Zyklus }}$ & $00: 28: 04$ & $7,07 \mathrm{E}+07$ & $3,92 \mathrm{E}+09$ & 5,23 & 56 & $10,2 \%$ & $\pm 1,3 \%$ \\
\hline$\Delta t_{\text {isPh }}$ & 00:05:02 & $2,51 E+08$ & $1,98 \mathrm{E}+10$ & 5,27 & 77 & $13,0 \%$ & $\pm 1,0 \%$ \\
\hline$\overline{\dot{q}}_{\text {Zyklus }}$ & 30 & $6,21 E+08$ & $6,24 \mathrm{E}+10$ & 5,28 & 97 & $15,1 \%$ & $\pm 0,6 \%$ \\
\hline$\overline{\dot{q}}_{\mathrm{isPh}}$ & 51 & $1,25 E+09$ & $1,52 E+11$ & 5,28 & 117 & $15,3 \%$ & $\pm 0,7 \%$ \\
\hline$\dot{q}_{\text {Impuls }}$ & 343,3 & $2,20 E+09$ & $3,16 \mathrm{E}+11$ & 5,27 & 140 & $15,1 \%$ & $\pm 0,8 \%$ \\
\hline$\Delta \vartheta_{L, \min }$ & 5,0 & $3,53 E+09$ & $5,85 \mathrm{E}+11$ & 5,26 & 163 & $14,1 \%$ & $\pm 1,0 \%$ \\
\hline$\Delta \vartheta_{L, \max }$ & 17,2 & $5,32 E+09$ & $9,98 \mathrm{E}+11$ & 5,24 & 182 & $14,6 \%$ & $\pm 0,8 \%$ \\
\hline$d \vartheta_{\mathrm{W}, \mathrm{L}} / d t_{1 \tau, \mathrm{Impuls}}$ & 0,086 & 7,57E+09 & $1,60 \mathrm{E}+12$ & 5,24 & 207 & $13,0 \%$ & $\pm 0,7 \%$ \\
\hline$d \vartheta_{\mathrm{W}, \mathrm{L}} / d t_{1 \tau, \text { Auskühlung }}$ & $-0,011$ & $1,04 \mathrm{E}+10$ & $2,43 \mathrm{E}+12$ & 5,24 & 233 & $12,0 \%$ & $\pm 0,7 \%$ \\
\hline \multicolumn{2}{|c|}{$\Delta t_{\text {Impuls }}=67 \mathrm{~s}, \Delta t_{\text {Auskühlung }}=1.094 \mathrm{~s}$} & $1,12 \mathrm{E}+07$ & $3,56 \mathrm{E}+08$ & 5,62 & 36 & $4,7 \%$ & $\pm 1,0 \%$ \\
\hline$\Delta t_{\text {Zyklus }}$ & $00: 19: 20$ & $9,59 E+07$ & $5,62 E+09$ & 5,72 & 60 & $12,8 \%$ & $\pm 0,5 \%$ \\
\hline$\Delta t_{\mathrm{isPh}}$ & 00:03:46 & $3,40 E+08$ & $2,83 \mathrm{E}+10$ & 5,79 & 82 & $14,9 \%$ & $\pm 1,0 \%$ \\
\hline$\overline{\dot{q}}_{\text {Zyklus }}$ & 43 & $8,40 E+08$ & $8,93 E+10$ & 5,79 & 103 & $16,3 \%$ & $\pm 0,6 \%$ \\
\hline$\overline{\dot{q}}_{\text {isPh }}$ & 72 & $1,69 \mathrm{E}+09$ & $2,17 \mathrm{E}+11$ & 5,78 & 125 & $15,8 \%$ & $\pm 0,8 \%$ \\
\hline$\dot{q}_{\text {Impuls }}$ & 743,6 & $2,96 \mathrm{E}+09$ & $4,51 \mathrm{E}+11$ & 5,76 & 148 & $14,9 \%$ & $\pm 1,3 \%$ \\
\hline$\Delta \vartheta_{\mathrm{L}, \min }$ & 8,2 & $4,75 E+09$ & $8,35 \mathrm{E}+11$ & 5,74 & 173 & $13,8 \%$ & $\pm 1,3 \%$ \\
\hline$\Delta \vartheta_{L, \max }$ & 21,2 & $7,14 \mathrm{E}+09$ & $1,42 \mathrm{E}+12$ & 5,70 & 196 & $13,7 \%$ & $\pm 1,2 \%$ \\
\hline$d \vartheta_{\mathrm{W}, \mathrm{L}} / d t_{1 \tau, \mathrm{Impuls}}$ & 0,191 & $1,02 \mathrm{E}+10$ & $2,28 \mathrm{E}+12$ & 5,70 & 225 & $12,1 \%$ & $\pm 1,1 \%$ \\
\hline$d \vartheta_{\mathrm{W}, \mathrm{L}} / d t_{1 \tau, \text { Auskühlung }}$ & $-0,016$ & $1,39 E+10$ & $3,48 \mathrm{E}+12$ & 5,69 & 253 & $11,3 \%$ & $\pm 1,0 \%$ \\
\hline
\end{tabular}


Tabelle A.1 (Fortsetzung): Ergebnisübersicht der Validierungsmessungen für zyklische Variationen

\begin{tabular}{|c|c|c|c|c|c|c|c|}
\hline \multicolumn{2}{|c|}{ Randbedingungen } & $\overline{\boldsymbol{G r}}_{\mathrm{x} / \mathrm{L}, \mathrm{zykl}}$ & $\overline{\boldsymbol{G}}_{\mathrm{x} / \mathrm{L}, \mathrm{Z} \mathrm{ykl} .}^{*}$ & $\Pi^{*}$ & $\overline{N u}_{\text {st }, \mathrm{x} / \mathrm{L}, \mathrm{zykl} .}$ & \multicolumn{2}{|c|}{$\bar{\Delta}_{\mathrm{is} / \mathrm{st}, \mathrm{isPh}} \stackrel{S}{\text { (5 Wdh.) }}$} \\
\hline \multicolumn{2}{|c|}{$\bar{c} \Delta t_{\text {Impuls }}=60 \mathrm{~s}, \Delta t_{\text {Auskühlung }}=1.301 \mathrm{~s}$} & $9,76 \mathrm{E}+06$ & $3,02 E+08$ & 6,66 & 34 & $5,0 \%$ & $\pm 1,7 \%$ \\
\hline$\Delta t_{\text {Zyklus }}$ & $00: 22: 40$ & $8,34 \mathrm{E}+07$ & $4,77 \mathrm{E}+09$ & 6,78 & 57 & $12,5 \%$ & $\pm 1,2 \%$ \\
\hline$\Delta t_{\text {isPh }}$ & 00:04:05 & $2,96 \mathrm{E}+08$ & $2,41 \mathrm{E}+10$ & 6,87 & 79 & $15,8 \%$ & $\pm 0,8 \%$ \\
\hline$\overline{\dot{q}}_{\text {Zyklus }}$ & 36 & $7,31 \mathrm{E}+08$ & $7,58 \mathrm{E}+10$ & 6,88 & 99 & $17,1 \%$ & $\pm 0,3 \%$ \\
\hline$\overline{\dot{q}}_{\text {isPh }}$ & 65 & $1,47 E+09$ & $1,85 \mathrm{E}+11$ & 6,86 & 120 & $17,2 \%$ & $\pm 0,2 \%$ \\
\hline$\dot{q}_{\text {Impuls }}$ & 828,0 & $2,58 \mathrm{E}+09$ & $3,83 \mathrm{E}+11$ & 6,83 & 143 & $16,7 \%$ & $\pm 0,5 \%$ \\
\hline$\Delta \vartheta_{\mathrm{L}, \min }$ & 6,6 & $4,14 \mathrm{E}+09$ & $7,09 \mathrm{E}+11$ & 6,81 & 167 & $15,5 \%$ & $\pm 0,8 \%$ \\
\hline$\Delta \vartheta_{L, \max }$ & 19,6 & $6,22 E+09$ & $1,21 \mathrm{E}+12$ & 6,76 & 188 & $15,6 \%$ & $\pm 0,8 \%$ \\
\hline$d \vartheta_{\mathrm{W}, \mathrm{L}} / d t_{1 \tau, \mathrm{Impuls}}$ & 0,216 & $8,86 \mathrm{E}+09$ & $1,94 \mathrm{E}+12$ & 6,76 & 214 & $14,6 \%$ & $\pm 0,9 \%$ \\
\hline$d \vartheta_{\mathrm{W}, \mathrm{L}} / d t_{1 \tau, \text { Auskühlung }}$ & $-0,014$ & $1,21 \mathrm{E}+10$ & $2,95 \mathrm{E}+12$ & 6,75 & 241 & $13,7 \%$ & $\pm 0,9 \%$ \\
\hline \multicolumn{2}{|c|}{$\Delta t_{\text {Impuls }}=36 \mathrm{~s}, \Delta t_{\text {Auskühlung }}=1.030 \mathrm{~s}$} & $1,21 \mathrm{E}+07$ & $3,85 \mathrm{E}+08$ & 7,30 & 35 & $6,7 \%$ & $\pm 3,2 \%$ \\
\hline$\Delta t_{\text {Zyklus }}$ & $00: 17: 44$ & $1,03 E+08$ & $6,08 \mathrm{E}+09$ & 7,47 & 59 & $13,2 \%$ & $\pm 1,8 \%$ \\
\hline$\Delta t_{\mathrm{isPh}}$ & 00:03:35 & $3,65 E+08$ & $3,07 E+10$ & 7,62 & 82 & $16,6 \%$ & $\pm 0,8 \%$ \\
\hline$\overline{\dot{q}}_{\text {Zyklus }}$ & 47 & $8,99 E+08$ & $9,66 \mathrm{E}+10$ & 7,61 & 103 & $17,8 \%$ & $\pm 0,2 \%$ \\
\hline$\overline{\dot{q}}_{\text {isPh }}$ & 80 & $1,80 E+09$ & $2,35 E+11$ & 7,57 & 125 & $17,5 \%$ & $\pm 0,4 \%$ \\
\hline$\dot{q}_{\text {Impuls }}$ & $1.405,7$ & $3,17 E+09$ & $4,88 \mathrm{E}+11$ & 7,53 & 149 & $16,6 \%$ & $\pm 0,5 \%$ \\
\hline$\Delta \vartheta_{L, \min }$ & 9,0 & $5,09 E+09$ & $9,03 E+11$ & 7,49 & 175 & $14,6 \%$ & $\pm 0,7 \%$ \\
\hline$\Delta \vartheta_{L, \max }$ & 22,7 & $7,64 \mathrm{E}+09$ & $1,54 \mathrm{E}+12$ & 7,40 & 199 & $14,4 \%$ & $\pm 1,0 \%$ \\
\hline$d \vartheta_{\mathrm{W}, \mathrm{L}} / d t_{1 \tau, \mathrm{Impuls}}$ & 0,362 & $1,09 \mathrm{E}+10$ & $2,47 \mathrm{E}+12$ & 7,40 & 227 & $13,3 \%$ & $\pm 1,3 \%$ \\
\hline$d \vartheta_{\mathrm{W}, \mathrm{L}} / d t_{1 \tau, \text { Auskühlung }}$ & $-0,017$ & $1,48 \mathrm{E}+10$ & $3,76 \mathrm{E}+12$ & 7,38 & 255 & $12,6 \%$ & $\pm 1,2 \%$ \\
\hline \multicolumn{2}{|c|}{$\Delta t_{\text {lmpuls }}=55 \mathrm{~s}, \Delta t_{\text {Auskühlung }}=1.511 \mathrm{~s}$} & $8,76 E+06$ & $2,66 \mathrm{E}+08$ & 7,69 & 34 & $6,6 \%$ & $\pm 1,0 \%$ \\
\hline$\Delta t_{\text {zyklus }}$ & 00:26:06 & $7,49 E+07$ & $4,20 \mathrm{E}+09$ & 7,84 & 57 & $14,0 \%$ & $\pm 1,3 \%$ \\
\hline$\Delta t_{\text {isPh }}$ & 00:04:07 & $2,66 \mathrm{E}+08$ & $2,12 \mathrm{E}+10$ & 7,95 & 78 & $17,0 \%$ & $\pm 1,2 \%$ \\
\hline$\overline{\dot{q}}_{\text {Zyklus }}$ & 32 & $6,57 E+08$ & $6,69 \mathrm{E}+10$ & 7,96 & 97 & $18,4 \%$ & $\pm 0,5 \%$ \\
\hline$\overline{\dot{q}}_{\text {isPh }}$ & 61 & $1,32 E+09$ & $1,63 E+11$ & 7,93 & 118 & $18,7 \%$ & $\pm 0,5 \%$ \\
\hline$\dot{q}_{\text {Impuls }}$ & 893,3 & $2,33 E+09$ & $3,38 \mathrm{E}+11$ & 7,90 & 141 & $18,0 \%$ & $\pm 0,7 \%$ \\
\hline$\Delta \vartheta_{\mathrm{L}, \min }$ & 5,5 & $3,74 \mathrm{E}+09$ & $6,26 \mathrm{E}+11$ & 7,87 & 164 & $16,6 \%$ & $\pm 1,3 \%$ \\
\hline$\Delta \vartheta_{\mathrm{L}, \max }$ & 18,8 & $5,63 E+09$ & $1,07 \mathrm{E}+12$ & 7,81 & 183 & $17,0 \%$ & $\pm 1,4 \%$ \\
\hline$d \vartheta_{\mathrm{W}, \mathrm{L}} / d t_{1 \tau, \mathrm{Impuls}}$ & 0,234 & $8,02 E+09$ & $1,71 \mathrm{E}+12$ & 7,81 & 208 & $16,2 \%$ & $\pm 1,5 \%$ \\
\hline$d \vartheta_{\mathrm{W}, \mathrm{L}} / d t_{1 \tau, \text { Auskühlung }}$ & $-0,013$ & $1,10 E+10$ & $2,61 \mathrm{E}+12$ & 7,80 & 233 & $15,4 \%$ & $\pm 1,3 \%$ \\
\hline \multicolumn{2}{|c|}{$\Delta t_{\text {Impuls }}=46 \mathrm{~s}, \Delta t_{\text {Auskühlung }}=1.442 \mathrm{~s}$} & $1,12 \mathrm{E}+07$ & $3,55 \mathrm{E}+08$ & 8,50 & 36 & $6,7 \%$ & $\pm 1,6 \%$ \\
\hline$\Delta t_{\text {zyklus }}$ & $00: 24: 47$ & $9,54 \mathrm{E}+07$ & $5,60 E+09$ & 8,70 & 60 & $13,5 \%$ & $\pm 0,1 \%$ \\
\hline$\Delta t_{\text {isPh }}$ & 00:03:38 & $3,39 E+08$ & $2,83 \mathrm{E}+10$ & 8,88 & 82 & $17,1 \%$ & $\pm 0,7 \%$ \\
\hline$\overline{\dot{q}}_{\text {Zyklus }}$ & 43 & $8,36 \mathrm{E}+08$ & $8,91 \mathrm{E}+10$ & 8,86 & 103 & $18,6 \%$ & $\pm 0,4 \%$ \\
\hline$\overline{\dot{q}}_{\text {isPh }}$ & 87 & $1,68 \mathrm{E}+09$ & $2,17 E+11$ & 8,82 & 125 & $18,8 \%$ & $\pm 0,4 \%$ \\
\hline$\dot{q}_{\text {Impuls }}$ & $1.405,3$ & $2,95 \mathrm{E}+09$ & $4,50 E+11$ & 8,77 & 149 & $17,5 \%$ & $\pm 0,4 \%$ \\
\hline$\Delta \vartheta_{L, \min }$ & 6,9 & $4,73 E+09$ & $8,33 E+11$ & 8,72 & 174 & $16,3 \%$ & $\pm 0,5 \%$ \\
\hline$\Delta \vartheta_{L, \max }$ & 24,3 & $7,09 E+09$ & $1,42 E+12$ & 8,61 & 198 & $16,4 \%$ & $\pm 0,9 \%$ \\
\hline$d \vartheta_{\mathrm{W}, \mathrm{L}} / d t_{1 \tau, \mathrm{Impuls}}$ & 0,368 & $1,01 \mathrm{E}+10$ & $2,28 \mathrm{E}+12$ & 8,61 & 227 & $15,5 \%$ & $\pm 1,5 \%$ \\
\hline$d \vartheta_{\mathrm{W}, \mathrm{L}} / d t_{1 \tau, \text { Auskühlung }}$ & $-0,018$ & $1,38 \mathrm{E}+10$ & $3,47 \mathrm{E}+12$ & 8,60 & 256 & $14,9 \%$ & $\pm 1,6 \%$ \\
\hline
\end{tabular}


Tabelle A.1 (Fortsetzung): Ergebnisübersicht der Validierungsmessungen für zyklische Variationen

\begin{tabular}{|c|c|c|c|c|c|c|c|}
\hline \multicolumn{2}{|c|}{ Randbedingungen } & $\overline{\boldsymbol{G r}}_{\mathrm{x} / \mathrm{L}, \mathrm{zykl}}$ & $\overline{\boldsymbol{G r}}_{\mathrm{x} / \mathrm{L}, \mathrm{Zyk} \mathrm{l}}^{*}$ & $\Pi^{*}$ & $\overline{N u}_{\text {st, } \mathrm{x} / \mathrm{L}, \mathrm{zyk} \mathbf{}}$ & \multicolumn{2}{|c|}{$\begin{array}{cc}\bar{\Delta}_{\text {is/st,isPh }} & s \\
\text { (5 Wdh.) }\end{array}$} \\
\hline \multicolumn{2}{|c|}{$\Delta t_{\text {Impuls }}=51 \mathrm{~s}, \Delta t_{\text {Auskühlung }}=1.734 \mathrm{~s}$} & $7,83 \mathrm{E}+06$ & $2,36 \mathrm{E}+08$ & 8,78 & 33 & $11,1 \%$ & $\pm 1,5 \%$ \\
\hline$\Delta t_{\text {Zyklus }}$ & 00:29:44 & $6,70 \mathrm{E}+07$ & $3,72 E+09$ & 8,95 & 56 & $16,1 \%$ & $\pm 0,8 \%$ \\
\hline$\Delta t_{\text {isPh }}$ & 00:04:02 & $2,38 \mathrm{E}+08$ & $1,88 \mathrm{E}+10$ & 9,09 & 77 & $18,3 \%$ & $\pm 0,9 \%$ \\
\hline$\overline{\dot{q}}_{\text {Zyklus }}$ & 28 & $5,89 E+08$ & $5,92 \mathrm{E}+10$ & 9,10 & 97 & $19,9 \%$ & $\pm 0,8 \%$ \\
\hline$\overline{\dot{q}}_{\mathrm{isPh}}$ & 59 & $1,18 \mathrm{E}+09$ & $1,44 \mathrm{E}+11$ & 9,07 & 118 & $19,8 \%$ & $\pm 0,3 \%$ \\
\hline$\dot{q}_{\text {Impuls }}$ & 961,8 & $2,08 \mathrm{E}+09$ & $2,99 \mathrm{E}+11$ & 9,03 & 140 & $19,5 \%$ & $\pm 0,5 \%$ \\
\hline$\Delta \vartheta_{\mathrm{L}, \min }$ & 4,4 & $3,34 \mathrm{E}+09$ & $5,54 \mathrm{E}+11$ & 8,99 & 163 & $18,3 \%$ & $\pm 0,6 \%$ \\
\hline$\Delta \vartheta_{L, \max }$ & 18,0 & $5,03 E+09$ & $9,45 \mathrm{E}+11$ & 8,92 & 181 & $19,0 \%$ & $\pm 0,6 \%$ \\
\hline$d \vartheta_{\mathrm{W}, \mathrm{L}} / d t_{1 \tau, \mathrm{Impuls}}$ & 0,255 & $7,17 \mathrm{E}+09$ & $1,51 \mathrm{E}+12$ & 8,91 & 205 & $17,9 \%$ & $\pm 0,9 \%$ \\
\hline$d \vartheta_{\mathrm{W}, \mathrm{L}} / d t_{1 \tau, \text { Auskühlung }}$ & $-0,012$ & $9,82 \mathrm{E}+09$ & $2,31 \mathrm{E}+12$ & 8,90 & 230 & $17,1 \%$ & $\pm 1,0 \%$ \\
\hline \multicolumn{2}{|c|}{$\Delta t_{\text {lmpuls }}=56 \mathrm{~s}, \Delta t_{\text {Auskühlung }}=1.800 \mathrm{~s}$} & $1,00 \mathrm{E}+07$ & $3,15 \mathrm{E}+08$ & 9,12 & 35 & $8,2 \%$ & $\pm 2,0 \%$ \\
\hline$\Delta t_{\text {Zyklus }}$ & $00: 30: 54$ & $8,55 E+07$ & 4,97E+09 & 9,32 & 60 & $14,8 \%$ & $\pm 0,5 \%$ \\
\hline$\Delta t_{\text {isPh }}$ & 00:04:08 & $3,04 \mathrm{E}+08$ & $2,51 \mathrm{E}+10$ & 9,49 & 81 & $18,5 \%$ & $\pm 0,2 \%$ \\
\hline$\overline{\dot{q}}_{\text {Zyklus }}$ & 38 & $7,51 \mathrm{E}+08$ & $7,91 \mathrm{E}+10$ & 9,48 & 102 & $19,7 \%$ & $\pm 0,4 \%$ \\
\hline$\overline{\dot{q}}_{\text {isPh }}$ & 86 & $1,51 \mathrm{E}+09$ & $1,93 \mathrm{E}+11$ & 9,44 & 124 & $20,1 \%$ & $\pm 0,4 \%$ \\
\hline$\dot{q}_{\text {Impuls }}$ & $1.268,0$ & $2,65 E+09$ & $4,00 E+11$ & 9,39 & 148 & $19,7 \%$ & $\pm 0,7 \%$ \\
\hline$\Delta \vartheta_{L, \min }$ & 5,4 & $4,25 E+09$ & $7,40 E+11$ & 9,34 & 172 & $18,8 \%$ & $\pm 0,9 \%$ \\
\hline$\Delta \vartheta_{L, \max }$ & 24,3 & $6,38 \mathrm{E}+09$ & $1,26 \mathrm{E}+12$ & 9,24 & 194 & $19,2 \%$ & $\pm 1,1 \%$ \\
\hline$d \vartheta_{\mathrm{W}, \mathrm{L}} / d t_{1 \tau, \mathrm{Impuls}}$ & 0,333 & $9,08 \mathrm{E}+09$ & $2,02 \mathrm{E}+12$ & 9,24 & 222 & $18,2 \%$ & $\pm 1,1 \%$ \\
\hline$d \vartheta_{\mathrm{W}, \mathrm{L}} / d t_{1 \tau, \text { Auskühlung }}$ & $-0,017$ & $1,24 \mathrm{E}+10$ & $3,09 \mathrm{E}+12$ & 9,23 & 251 & $17,3 \%$ & $\pm 1,1 \%$ \\
\hline \multicolumn{2}{|c|}{$\Delta t_{\text {Impuls }}=45 \mathrm{~s}, \Delta t_{\text {Auskühlung }}=1.800 \mathrm{~s}$} & $7,72 E+06$ & $2,32 E+08$ & 9,62 & 33 & $9,2 \%$ & $\pm 1,2 \%$ \\
\hline$\Delta t_{\text {Zyklus }}$ & $00: 30: 44$ & $6,61 \mathrm{E}+07$ & $3,66 \mathrm{E}+09$ & 9,83 & 56 & $15,2 \%$ & $\pm 1,3 \%$ \\
\hline$\Delta t_{\mathrm{isPh}}$ & 00:04:09 & $2,35 E+08$ & $1,85 \mathrm{E}+10$ & 10,00 & 76 & $18,8 \%$ & $\pm 1,4 \%$ \\
\hline$\overline{\dot{q}}_{\text {Zyklus }}$ & 28 & $5,82 E+08$ & $5,84 \mathrm{E}+10$ & 9,99 & 95 & $20,5 \%$ & $\pm 0,9 \%$ \\
\hline$\overline{\dot{q}}_{\mathrm{isPh}}$ & 60 & $1,17 E+09$ & $1,42 E+11$ & 9,96 & 115 & $20,8 \%$ & $\pm 0,3 \%$ \\
\hline$\dot{q}_{\text {Impuls }}$ & $1.109,0$ & $2,06 E+09$ & $2,95 \mathrm{E}+11$ & 9,91 & 137 & $20,5 \%$ & $\pm 0,6 \%$ \\
\hline$\Delta \vartheta_{\mathrm{L}, \min }$ & 4,3 & $3,31 E+09$ & $5,46 E+11$ & 9,86 & 159 & $19,9 \%$ & $\pm 0,6 \%$ \\
\hline$\Delta \vartheta_{\mathrm{L}, \max }$ & 18,2 & $4,98 E+09$ & $9,32 E+11$ & 9,77 & 178 & $20,1 \%$ & $\pm 0,9 \%$ \\
\hline$d \vartheta_{\mathrm{W}, \mathrm{L}} / d t_{1 \tau, \mathrm{Impuls}}$ & 0,292 & $7,10 E+09$ & $1,49 E+12$ & 9,77 & 202 & $18,8 \%$ & $\pm 1,1 \%$ \\
\hline$d \vartheta_{\mathrm{W}, \mathrm{L}} / d t_{1 \tau, \text { Auskühlung }}$ & $-0,012$ & $9,72 \mathrm{E}+09$ & $2,28 \mathrm{E}+12$ & 9,75 & 228 & $17,8 \%$ & $\pm 1,3 \%$ \\
\hline \multicolumn{2}{|c|}{$\Delta t_{\text {Impuls }}=38 \mathrm{~s}, \Delta t_{\text {Auskühlung }}=1.800 \mathrm{~s}$} & $7,62 E+06$ & $2,30 \mathrm{E}+08$ & 10,51 & 32 & $11,1 \%$ & $\pm 1,8 \%$ \\
\hline$\Delta t_{\text {Zyklus }}$ & $00: 30: 36$ & $6,53 E+07$ & $3,62 E+09$ & 10,76 & 54 & $17,3 \%$ & $\pm 2,4 \%$ \\
\hline$\Delta t_{\text {isPh }}$ & 00:03:56 & $2,32 E+08$ & $1,83 E+10$ & 10,97 & 75 & $19,4 \%$ & $\pm 1,3 \%$ \\
\hline$\overline{\dot{q}}_{\text {Zyklus }}$ & 27 & $5,75 \mathrm{E}+08$ & $5,77 \mathrm{E}+10$ & 10,96 & 93 & $21,8 \%$ & $\pm 0,4 \%$ \\
\hline$\overline{\dot{q}}_{\text {isPh }}$ & 59 & $1,16 \mathrm{E}+09$ & $1,41 \mathrm{E}+11$ & 10,91 & 113 & $22,0 \%$ & $\pm 0,4 \%$ \\
\hline$\dot{q}_{\text {Impuls }}$ & $1.319,2$ & $2,04 \mathrm{E}+09$ & $2,92 E+11$ & 10,85 & 135 & $21,2 \%$ & $\pm 0,6 \%$ \\
\hline$\Delta \vartheta_{L, \min }$ & 4,2 & $3,27 E+09$ & $5,40 E+11$ & 10,79 & 157 & $19,9 \%$ & $\pm 1,2 \%$ \\
\hline$\Delta \vartheta_{L, \max }$ & 18,0 & $4,92 E+09$ & $9,21 \mathrm{E}+11$ & 10,66 & 177 & $19,5 \%$ & $\pm 1,1 \%$ \\
\hline$d \vartheta_{\mathrm{W}, \mathrm{L}} / d t_{1 \tau, \mathrm{Impuls}}$ & 0,347 & $7,02 E+09$ & $1,48 \mathrm{E}+12$ & 10,66 & 201 & $17,9 \%$ & $\pm 1,0 \%$ \\
\hline$d \vartheta_{\mathrm{W}, \mathrm{L}} / d t_{1 \tau, \text { Auskühlung }}$ & $-0,012$ & $9,60 E+09$ & $2,25 \mathrm{E}+12$ & 10,65 & 226 & $17,2 \%$ & $\pm 1,1 \%$ \\
\hline
\end{tabular}




\section{A.2 Ergebnisse der Validierungsmessungen für sprungartige Änderungen}

Tabelle A.2 zeigt die Ergebnisse der Validierungs-Versuchsreihen für sprungartig veränderte Randbedingungen (Kapitel 5.3.1). Die Randbedingungen werden hier im Format hh:mm:ss sowie in den Einheiten W/m², $\mathrm{K}, 1 / \mathrm{s}$ und $\mathrm{K} / \mathrm{s}$ angegeben.

Tabelle A.2: Ergebnisübersicht der Validierungsmessungen für sprungartige Änderungen

\begin{tabular}{|c|c|c|c|c|c|c|c|}
\hline \multicolumn{2}{|c|}{ Randbedingungen } & \multirow{2}{*}{\begin{tabular}{|c|}
$\overline{\boldsymbol{G r}}_{\mathrm{x} / \mathrm{L}, \mathrm{Spr}}$ \\
$3,09 \mathrm{E}+07$
\end{tabular}} & \multirow{2}{*}{$\frac{\boldsymbol{G} \boldsymbol{r}_{\mathrm{x} / \mathrm{L}, \mathrm{m}}}{2,08 \mathrm{E}+07}$} & \multirow{2}{*}{$\begin{array}{l}\text { П } \\
0,81\end{array}$} & \multirow{2}{*}{$\begin{array}{c}\overline{\boldsymbol{N u}}_{\text {st, } \mathbf{x} / \mathrm{L}, \mathrm{Spr}} \\
42\end{array}$} & \multicolumn{2}{|c|}{$\bar{\Delta}_{\mathrm{is} / \mathrm{st}, \mathrm{isPh}} \underset{\text { (5 Wdh.) }}{S}$} \\
\hline$\Delta \vartheta_{\text {Sprung }}=51 \mathrm{~K}$ & & & & & & $0,8 \%$ & $\pm 1,5 \%$ \\
\hline$\Delta t_{\text {Sprung }}$ & $00: 23: 44$ & $2,54 \mathrm{E}+08$ & $1,68 \mathrm{E}+08$ & 0,82 & 72 & $6,6 \%$ & $\pm 1,1 \%$ \\
\hline$\Delta t_{\text {isPh }}$ & $00: 15: 26$ & $8,72 E+08$ & $5,69 E+08$ & 0,82 & 99 & $9,7 \%$ & $\pm 0,7 \%$ \\
\hline$\overline{\dot{q}}_{\text {sprung }}$ & 168 & $2,09 E+09$ & $1,35 E+09$ & 0,83 & 125 & $10,2 \%$ & $\pm 1,1 \%$ \\
\hline$\overline{\dot{q}}_{\mathrm{isPh}}$ & 129 & $4,09 E+09$ & $2,64 \mathrm{E}+09$ & 0,83 & 154 & $9,7 \%$ & $\pm 1,9 \%$ \\
\hline$\dot{q}_{\max }$ & 316,2 & $7,09 E+09$ & $4,57 E+09$ & 0,82 & 189 & $8,8 \%$ & $\pm 1,8 \%$ \\
\hline$\Delta \vartheta_{L, \min }$ & 0,0 & $1,12 \mathrm{E}+10$ & $7,26 \mathrm{E}+09$ & 0,82 & 227 & $7,5 \%$ & $\pm 1,5 \%$ \\
\hline$\Delta \vartheta_{\mathrm{L}, \max }$ & 50,1 & $1,68 E+10$ & $1,08 \mathrm{E}+10$ & 0,82 & 263 & $7,3 \%$ & $\pm 1,1 \%$ \\
\hline$\dot{q}_{\max } /\left(\Delta \vartheta_{\text {Sprung }} \cdot C_{\mathrm{W}} / A_{\mathrm{W}}\right)$ & $1,74 \mathrm{E}-03$ & $2,38 \mathrm{E}+10$ & $1,54 \mathrm{E}+10$ & 0,82 & 300 & $7,1 \%$ & $\pm 0,9 \%$ \\
\hline$d \vartheta_{\mathrm{W}, \mathrm{L}} / d t_{1 \mathrm{t}, \mathrm{Sprung}}$ & 0,065 & $3,26 E+10$ & $2,12 \mathrm{E}+10$ & 0,82 & 337 & $6,9 \%$ & $\pm 0,8 \%$ \\
\hline$\Delta \vartheta_{\text {Sprung }}=42 \mathrm{~K}$ & & $2,20 E+07$ & $1,81 \mathrm{E}+07$ & 1,59 & 41 & $8,1 \%$ & $\pm 0,6 \%$ \\
\hline$\Delta t_{\text {Sprung }}$ & $00: 05: 27$ & $1,78 \mathrm{E}+08$ & $1,46 E+08$ & 1,62 & 68 & $11,5 \%$ & $\pm 0,7 \%$ \\
\hline$\Delta t_{\text {isPh }}$ & 00:09:08 & $6,05 E+08$ & $4,97 E+08$ & 1,64 & 93 & $12,5 \%$ & $\pm 0,6 \%$ \\
\hline$\overline{\dot{q}}_{\text {sprung }}$ & 113 & $1,44 E+09$ & $1,18 \mathrm{E}+09$ & 1,64 & 116 & $13,4 \%$ & $\pm 0,5 \%$ \\
\hline$\overline{\dot{q}}_{\text {isPh }}$ & 149 & $2,82 E+09$ & $2,31 E+09$ & 1,64 & 141 & $13,9 \%$ & $\pm 0,7 \%$ \\
\hline$\dot{q}_{\max }$ & 587,0 & $4,88 E+09$ & $3,99 E+09$ & 1,63 & 170 & $13,7 \%$ & $\pm 0,8 \%$ \\
\hline$\Delta \vartheta_{L, \min }$ & 0,0 & $7,75 E+09$ & $6,34 \mathrm{E}+09$ & 1,63 & 202 & $13,1 \%$ & $\pm 0,9 \%$ \\
\hline$\Delta \vartheta_{L, \max }$ & 41,4 & $1,16 E+10$ & $9,47 E+09$ & 1,62 & 235 & $12,5 \%$ & $\pm 0,7 \%$ \\
\hline$\dot{q}_{\max } /\left(\Delta \vartheta_{\text {Sprung }} \cdot C_{\mathrm{W}} / A_{\mathrm{W}}\right)$ & 0,004 & $1,65 E+10$ & $1,35 \mathrm{E}+10$ & 1,62 & 270 & $12,0 \%$ & $\pm 0,5 \%$ \\
\hline$d \vartheta_{\mathrm{W}, \mathrm{L}} / d t_{1 \mathrm{~T}, \mathrm{Sprung}}$ & 0,143 & $2,26 E+10$ & $1,85 \mathrm{E}+10$ & 1,62 & 305 & $11,8 \%$ & $\pm 0,5 \%$ \\
\hline$\Delta \vartheta_{\text {Sprung }}=33 \mathrm{~K}$ & & $1,75 E+07$ & $1,51 \mathrm{E}+07$ & 2,44 & 40 & $11,8 \%$ & $\pm 1,1 \%$ \\
\hline$\Delta t_{\text {Sprung }}$ & 00:03:00 & $1,41 E+08$ & $1,22 \mathrm{E}+08$ & 2,48 & 65 & $15,2 \%$ & $\pm 0,8 \%$ \\
\hline$\Delta t_{\text {isPh }}$ & 00:07:01 & $4,78 E+08$ & $4,12 E+08$ & 2,52 & 89 & $16,2 \%$ & $\pm 0,7 \%$ \\
\hline$\overline{\dot{q}}_{\text {sprung }}$ & 91 & $1,14 \mathrm{E}+09$ & $9,78 \mathrm{E}+08$ & 2,52 & 111 & $17,1 \%$ & $\pm 0,8 \%$ \\
\hline$\overline{\dot{q}}_{\text {isPh }}$ & 125 & $2,22 E+09$ & $1,91 E+09$ & 2,52 & 135 & $17,6 \%$ & $\pm 1,0 \%$ \\
\hline$\dot{q}_{\max }$ & 766,5 & $3,84 E+09$ & $3,30 E+09$ & 2,51 & 161 & $17,8 \%$ & $\pm 1,2 \%$ \\
\hline$\Delta \vartheta_{\mathrm{L}, \min }$ & 0,0 & $6,10 E+09$ & $5,25 \mathrm{E}+09$ & 2,50 & 189 & $17,6 \%$ & $\pm 1,4 \%$ \\
\hline$\Delta \vartheta_{L, \max }$ & 32,6 & $9,12 E+09$ & $7,84 \mathrm{E}+09$ & 2,48 & 218 & $17,3 \%$ & $\pm 1,4 \%$ \\
\hline$\dot{q}_{\max } /\left(\Delta \vartheta_{\text {Sprung }} \cdot C_{\mathrm{W}} / A_{\mathrm{W}}\right)$ & 0,006 & $1,30 \mathrm{E}+10$ & $1,12 \mathrm{E}+10$ & 2,48 & 251 & $16,6 \%$ & $\pm 1,3 \%$ \\
\hline$d \vartheta_{\mathrm{W}, \mathrm{L}} / d t_{1 \mathrm{t}, \mathrm{Sprung}}$ & 0,196 & $1,78 \mathrm{E}+10$ & $1,53 \mathrm{E}+10$ & 2,48 & 284 & $16,0 \%$ & $\pm 1,1 \%$ \\
\hline
\end{tabular}


Tabelle A.2 (Fortsetzung): Ergebnisübersicht der Validierungsmessungen für sprungartige Änderungen

\begin{tabular}{|c|c|c|c|c|c|c|c|}
\hline \multicolumn{2}{|l|}{ Randbedingungen } & \multirow{2}{*}{$\begin{array}{c}\overline{\boldsymbol{G}}_{\mathrm{x} / \mathrm{L}, \mathrm{Spr}} . \\
1,46 \mathrm{E}+07\end{array}$} & \multirow{2}{*}{$\begin{array}{l}\boldsymbol{G} \boldsymbol{r}_{\mathrm{x} / \mathrm{L}, \mathrm{m}} \\
1,29 \mathrm{E}+07\end{array}$} & \multirow{2}{*}{$\frac{\pi}{3,28}$} & \multirow{2}{*}{$\frac{\overline{\boldsymbol{N u}}_{\text {st, } \mathrm{x} / \mathrm{L}, \mathrm{Spr} .}}{38}$} & \multicolumn{2}{|c|}{$\bar{\Delta}_{\text {is/st,isPh }} \begin{array}{c}S \\
\text { (5 Wdh.) }\end{array}$} \\
\hline$\Delta \vartheta_{\text {Sprung }}=27 \mathrm{~K}$ & & & & & & $14,1 \%$ & $\pm 1,7 \%$ \\
\hline$\Delta t_{\text {Sprung }}$ & 00:02:02 & $1,17 \mathrm{E}+08$ & $1,03 E+08$ & 3,35 & 63 & $18,1 \%$ & $\pm 0,7 \%$ \\
\hline$\Delta t_{\text {isPh }}$ & 00:05:32 & $3,96 \mathrm{E}+08$ & $3,48 \mathrm{E}+08$ & 3,40 & 87 & $18,8 \%$ & $\pm 0,7 \%$ \\
\hline$\overline{\dot{q}}_{\text {sprung }}$ & 69 & $9,43 E+08$ & $8,27 \mathrm{E}+08$ & 3,41 & 108 & $19,0 \%$ & $\pm 0,5 \%$ \\
\hline$\overline{\dot{q}}_{\text {isPh }}$ & 102 & $1,83 E+09$ & $1,61 \mathrm{E}+09$ & 3,40 & 132 & $19,6 \%$ & $\pm 0,3 \%$ \\
\hline$\dot{q}_{\max }$ & 893,3 & $3,17 E+09$ & $2,79 E+09$ & 3,38 & 157 & $19,6 \%$ & $\pm 0,7 \%$ \\
\hline$\Delta \vartheta_{L, \min }$ & 0,0 & $5,04 \mathrm{E}+09$ & $4,44 \mathrm{E}+09$ & 3,37 & 183 & $19,3 \%$ & $\pm 0,8 \%$ \\
\hline$\Delta \vartheta_{\mathrm{L}, \max }$ & 26,6 & $7,55 E+09$ & $6,63 E+09$ & 3,34 & 208 & $19,4 \%$ & $\pm 0,9 \%$ \\
\hline$\dot{q}_{\max } /\left(\Delta \vartheta_{\text {sprung }} \cdot C_{\mathrm{W}} / A_{\mathrm{W}}\right)$ & 0,009 & $1,08 \mathrm{E}+10$ & $9,44 E+09$ & 3,34 & 239 & $18,7 \%$ & $\pm 0,9 \%$ \\
\hline$d \vartheta_{\mathrm{W}, \mathrm{L}} / d t_{1 \mathrm{t}, \mathrm{Sprung}}$ & 0,236 & $1,48 \mathrm{E}+10$ & $1,29 E+10$ & 3,34 & 271 & $17,8 \%$ & $\pm 1,0 \%$ \\
\hline$\Delta \vartheta_{\text {Sprung }}=21 \mathrm{~K}$ & & $1,16 \mathrm{E}+07$ & $1,04 \mathrm{E}+07$ & 4,17 & 36 & $14,4 \%$ & $\pm 0,3 \%$ \\
\hline$\Delta t_{\text {Sprung }}$ & 00:01:30 & $9,34 \mathrm{E}+07$ & $8,29 E+07$ & 4,26 & 61 & $17,7 \%$ & $\pm 0,8 \%$ \\
\hline$\Delta t_{\text {isPh }}$ & 00:05:14 & $3,15 E+08$ & $2,80 E+08$ & 4,33 & 83 & $18,4 \%$ & $\pm 1,3 \%$ \\
\hline$\overline{\dot{q}}_{\text {sprung }}$ & 46 & $7,51 \mathrm{E}+08$ & $6,66 \mathrm{E}+08$ & 4,34 & 104 & $19,5 \%$ & $\pm 1,2 \%$ \\
\hline$\overline{\dot{q}}_{\text {isPh }}$ & 78 & $1,46 E+09$ & $1,30 E+09$ & 4,33 & 126 & $20,5 \%$ & $\pm 0,6 \%$ \\
\hline$\dot{q}_{\max }$ & 937,2 & $2,52 E+09$ & $2,25 E+09$ & 4,31 & 150 & $20,5 \%$ & $\pm 0,7 \%$ \\
\hline$\Delta \vartheta_{L, \min }$ & 0,0 & $4,01 E+09$ & $3,57 E+09$ & 4,29 & 175 & $20,3 \%$ & $\pm 0,3 \%$ \\
\hline$\Delta \vartheta_{L, \max }$ & 20,7 & $6,01 E+09$ & $5,33 E+09$ & 4,26 & 197 & $20,5 \%$ & $\pm 0,3 \%$ \\
\hline$\dot{q}_{\max } /\left(\Delta \vartheta_{\text {Sprung }} \cdot C_{\mathrm{W}} / A_{\mathrm{W}}\right)$ & 0,013 & $8,56 \mathrm{E}+09$ & $7,59 \mathrm{E}+09$ & 4,25 & 226 & $19,7 \%$ & $\pm 0,5 \%$ \\
\hline$d \vartheta_{\mathrm{W}, \mathrm{L}} / d t_{1 \mathrm{r}, \mathrm{Sprung}}$ & 0,252 & $1,17 \mathrm{E}+10$ & $1,04 \mathrm{E}+10$ & 4,25 & 255 & $18,8 \%$ & $\pm 0,5 \%$ \\
\hline$\Delta \vartheta_{\text {Sprung }}=18 \mathrm{~K}$ & & $1,01 \mathrm{E}+07$ & $9,05 E+06$ & 5,15 & 36 & $14,0 \%$ & $\pm 1,7 \%$ \\
\hline$\Delta t_{\text {Sprung }}$ & 00:01:10 & $8,12 \mathrm{E}+07$ & $7,22 \mathrm{E}+07$ & 5,27 & 59 & $18,7 \%$ & $\pm 2,2 \%$ \\
\hline$\Delta t_{\mathrm{isPh}}$ & 00:04:51 & $2,73 E+08$ & $2,44 E+08$ & 5,36 & 81 & $20,7 \%$ & $\pm 1,2 \%$ \\
\hline$\overline{\dot{q}}_{\text {Sprung }}$ & 35 & $6,51 E+08$ & $5,79 E+08$ & 5,37 & 101 & $21,6 \%$ & $\pm 0,9 \%$ \\
\hline$\overline{\dot{q}}_{\text {isPh }}$ & 66 & $1,26 \mathrm{E}+09$ & $1,13 E+09$ & 5,35 & 121 & $22,5 \%$ & $\pm 0,7 \%$ \\
\hline$\dot{q}_{\max }$ & $1.031,2$ & $2,18 E+09$ & $1,95 \mathrm{E}+09$ & 5,33 & 143 & $22,2 \%$ & $\pm 0,4 \%$ \\
\hline$\Delta \vartheta_{L, \min }$ & 0,0 & $3,47 E+09$ & $3,10 E+09$ & 5,30 & 167 & $21,9 \%$ & $\pm 1,0 \%$ \\
\hline$\Delta \vartheta_{L, \max }$ & 17,6 & $5,20 \mathrm{E}+09$ & $4,63 E+09$ & 5,26 & 187 & $22,2 \%$ & $\pm 1,8 \%$ \\
\hline$\dot{q}_{\max } /\left(\Delta \vartheta_{\text {Sprung }} \cdot C_{\mathrm{W}} / A_{\mathrm{W}}\right)$ & 0,016 & $7,42 E+09$ & $6,59 E+09$ & 5,25 & 214 & $21,6 \%$ & $\pm 1,9 \%$ \\
\hline$d \vartheta_{\mathrm{W}, \mathrm{L}} / d t_{1 \mathrm{r}, \mathrm{Sprung}}$ & 0,279 & $1,02 \mathrm{E}+10$ & $9,04 \mathrm{E}+09$ & 5,25 & 242 & $20,6 \%$ & $\pm 1,7 \%$ \\
\hline$\Delta \vartheta_{\text {Sprung }}=15 \mathrm{~K}$ & & $8,52 E+06$ & $7,63 E+06$ & 6,05 & 34 & $13,5 \%$ & $\pm 2,4 \%$ \\
\hline$\Delta t_{\text {Sprung }}$ & $00: 00: 58$ & $6,88 \mathrm{E}+07$ & $6,11 E+07$ & 6,19 & 57 & $18,4 \%$ & $\pm 2,1 \%$ \\
\hline$\Delta t_{\text {isPh }}$ & 00:05:02 & $2,31 E+08$ & $2,07 E+08$ & 6,30 & 78 & $19,8 \%$ & $\pm 1,4 \%$ \\
\hline$\overline{\dot{q}}_{\text {sprung }}$ & 22 & $5,51 E+08$ & $4,91 E+08$ & 6,31 & 97 & $21,3 \%$ & $\pm 0,8 \%$ \\
\hline$\overline{\dot{q}}_{\mathrm{isPh}}$ & 55 & $1,07 E+09$ & $9,58 \mathrm{E}+08$ & 6,29 & 117 & $22,8 \%$ & $\pm 0,7 \%$ \\
\hline$\dot{q}_{\max }$ & $1.054,3$ & $1,85 \mathrm{E}+09$ & $1,66 \mathrm{E}+09$ & 6,26 & 139 & $23,7 \%$ & $\pm 1,0 \%$ \\
\hline$\Delta \vartheta_{L, \min }$ & 0,0 & $2,93 E+09$ & $2,63 E+09$ & 6,23 & 162 & $23,7 \%$ & $\pm 1,1 \%$ \\
\hline$\Delta \vartheta_{L, \max }$ & 14,7 & $4,41 E+09$ & $3,93 E+09$ & 6,17 & 181 & $23,8 \%$ & $\pm 1,6 \%$ \\
\hline$\dot{q}_{\max } /\left(\Delta \vartheta_{\text {Sprung }} \cdot C_{\mathrm{W}} / A_{\mathrm{W}}\right)$ & 0,020 & $6,28 \mathrm{E}+09$ & $5,60 \mathrm{E}+09$ & 6,17 & 205 & $23,2 \%$ & $\pm 2,0 \%$ \\
\hline$d \vartheta_{\mathrm{W}, \mathrm{L}} / d t_{1 \tau, \text { Sprung }}$ & 0,285 & $8,62 E+09$ & $7,68 \mathrm{E}+09$ & 6,16 & 231 & $22,0 \%$ & $\pm 1,9 \%$ \\
\hline
\end{tabular}


Tabelle A.2 (Fortsetzung): Ergebnisübersicht der Validierungsmessungen für sprungartige Änderungen

\begin{tabular}{|c|c|c|c|c|c|c|c|}
\hline \multicolumn{2}{|l|}{ Randbedingungen } & \multirow{2}{*}{$\frac{\overline{\boldsymbol{G r}}_{\mathbf{x} / \mathrm{L}, \mathrm{Spr}}}{\mathrm{7,35 \textrm {E } + 0 6}}$} & \multirow{2}{*}{$\begin{array}{c}\boldsymbol{G} \boldsymbol{r}_{\mathrm{x} / \mathrm{L}, \mathrm{m}} \\
6,67 \mathrm{E}+06\end{array}$} & \multirow{2}{*}{$\frac{\Pi}{6,80}$} & \multirow{2}{*}{\begin{tabular}{|c|}
$\overline{\boldsymbol{N u}}_{\text {st, } \mathbf{x} / \mathrm{L}, \mathrm{Spr}}$ \\
33
\end{tabular}} & \multicolumn{2}{|c|}{$\bar{\Delta}_{\text {is/st,isph }} \begin{array}{c}s \\
(5 \text { Wdh. })\end{array}$} \\
\hline$\Delta \vartheta_{\text {Sprung }}=13 \mathrm{~K}$ & & & & & & $15,9 \%$ & $\pm 1,7 \%$ \\
\hline$\Delta t_{\text {Sprung }}$ & 00:00:50 & $5,94 \mathrm{E}+07$ & $5,34 \mathrm{E}+07$ & 6,95 & 55 & $20,7 \%$ & $\pm 1,6 \%$ \\
\hline$\Delta t_{\text {isPh }}$ & 00:04:30 & $1,99 \mathrm{E}+08$ & $1,80 \mathrm{E}+08$ & 7,08 & 75 & $22,1 \%$ & $\pm 1,2 \%$ \\
\hline$\overline{\dot{q}}_{\text {sprung }}$ & 15 & $4,76 E+08$ & $4,27 E+08$ & 7,08 & 93 & $23,4 \%$ & $\pm 0,6 \%$ \\
\hline$\overline{\dot{q}}_{\text {isPh }}$ & 45 & $9,20 E+08$ & $8,34 \mathrm{E}+08$ & 7,06 & 112 & $25,5 \%$ & $\pm 0,6 \%$ \\
\hline$\dot{q}_{\max }$ & $1.053,8$ & $1,59 E+09$ & $1,44 \mathrm{E}+09$ & 7,03 & 134 & $25,5 \%$ & $\pm 1,2 \%$ \\
\hline$\Delta \vartheta_{\mathrm{L}, \min }$ & 0,0 & $2,53 E+09$ & $2,29 E+09$ & 7,00 & 157 & $24,7 \%$ & $\pm 1,9 \%$ \\
\hline$\Delta \vartheta_{L, \max }$ & 12,7 & $3,80 E+09$ & $3,42 E+09$ & 6,93 & 175 & $23,8 \%$ & $\pm 1,6 \%$ \\
\hline$\dot{q}_{\max } /\left(\Delta \vartheta_{\text {sprung }} \cdot C_{\mathrm{W}} / A_{\mathrm{W}}\right)$ & 0,023 & $5,43 E+09$ & $4,87 E+09$ & 6,93 & 198 & $22,8 \%$ & $\pm 1,3 \%$ \\
\hline$d \vartheta_{\mathrm{W}, \mathrm{L}} / d t_{1 \mathrm{\tau}, \text { Sprung }}$ & 0,284 & 7,44E+09 & $6,69 E+09$ & 6,92 & 222 & $21,2 \%$ & $\pm 1,4 \%$ \\
\hline$\Delta \vartheta_{\text {Sprung }}=11 \mathrm{~K}$ & & $6,25 E+06$ & $5,66 E+06$ & 7,91 & 32 & $17,5 \%$ & $\pm 2,9 \%$ \\
\hline$\Delta t_{\text {Sprung }}$ & $00: 00: 43$ & $5,07 E+07$ & $4,52 E+07$ & 8,09 & 53 & $22,0 \%$ & $\pm 3,4 \%$ \\
\hline$\Delta t_{\text {isPh }}$ & $00: 04: 17$ & $1,70 E+08$ & $1,53 E+08$ & 8,24 & 72 & $24,1 \%$ & $\pm 1,2 \%$ \\
\hline$\overline{\dot{q}}_{\text {Sprung }}$ & 7 & $4,07 E+08$ & $3,64 \mathrm{E}+08$ & 8,25 & 89 & $23,7 \%$ & $\pm 0,9 \%$ \\
\hline$\dot{\dot{q}}_{\text {isPh }}$ & 38 & $7,86 \mathrm{E}+08$ & $7,12 E+08$ & 8,22 & 108 & $26,3 \%$ & $\pm 0,6 \%$ \\
\hline$\dot{q}_{\max }$ & $1.076,7$ & $1,36 \mathrm{E}+09$ & $1,23 E+09$ & 8,18 & 130 & $26,0 \%$ & $\pm 0,9 \%$ \\
\hline$\Delta \vartheta_{\mathrm{L}, \min }$ & 0,0 & $2,16 E+09$ & $1,95 \mathrm{E}+09$ & 8,14 & 152 & $25,8 \%$ & $\pm 0,8 \%$ \\
\hline$\Delta \vartheta_{L, \max }$ & 10,7 & $3,25 E+09$ & $2,92 E+09$ & 8,07 & 169 & $26,5 \%$ & $\pm 1,5 \%$ \\
\hline$\dot{q}_{\max } /\left(\Delta \vartheta_{\text {sprung }} \cdot C_{\mathrm{W}} / A_{\mathrm{W}}\right)$ & 0,028 & $4,64 \mathrm{E}+09$ & $4,16 \mathrm{E}+09$ & 8,06 & 191 & $25,4 \%$ & $\pm 2,0 \%$ \\
\hline$d \vartheta_{\mathrm{W}, \mathrm{L}} / d t_{1 \mathrm{\tau}, \text { Sprung }}$ & 0,289 & $6,36 \mathrm{E}+09$ & $5,71 E+09$ & 8,05 & 214 & $24,4 \%$ & $\pm 2,2 \%$ \\
\hline$\Delta \vartheta_{\text {Sprung }}=10 \mathrm{~K}$ & & $5,77 E+06$ & $5,18 \mathrm{E}+06$ & 8,90 & 31 & $15,9 \%$ & $\pm 1,5 \%$ \\
\hline$\Delta t_{\text {Sprung }}$ & $00: 00: 38$ & $4,67 E+07$ & $4,13 E+07$ & 9,11 & 51 & $22,7 \%$ & $\pm 1,7 \%$ \\
\hline$\Delta t_{\text {isPh }}$ & $00: 04: 35$ & $1,56 \mathrm{E}+08$ & $1,40 E+08$ & 9,27 & 70 & $22,8 \%$ & $\pm 1,3 \%$ \\
\hline$\dot{\bar{q}}_{\text {Sprung }}$ & 7 & $3,75 E+08$ & $3,32 E+08$ & 9,28 & 87 & $25,0 \%$ & $\pm 0,7 \%$ \\
\hline$\overline{\dot{q}}_{\text {isPh }}$ & 34 & $7,24 \mathrm{E}+08$ & $6,51 E+08$ & 9,25 & 105 & $27,2 \%$ & $\pm 0,5 \%$ \\
\hline$\dot{q}_{\max }$ & $1.128,0$ & $1,25 \mathrm{E}+09$ & $1,13 E+09$ & 9,21 & 127 & $27,5 \%$ & $\pm 0,9 \%$ \\
\hline$\Delta \vartheta_{L, \min }$ & 0,0 & $1,99 \mathrm{E}+09$ & $1,79 E+09$ & 9,16 & 149 & $27,1 \%$ & $\pm 1,8 \%$ \\
\hline$\Delta \vartheta_{L, \max }$ & 9,7 & $2,99 E+09$ & $2,67 E+09$ & 9,07 & 164 & $27,5 \%$ & $\pm 1,4 \%$ \\
\hline$\dot{q}_{\max } /\left(\Delta \vartheta_{\text {sprung }} \cdot C_{\mathrm{W}} / A_{\mathrm{W}}\right)$ & 0,032 & $4,27 E+09$ & $3,80 E+09$ & 9,07 & 186 & $26,5 \%$ & $\pm 2,1 \%$ \\
\hline$d \vartheta_{\mathrm{W}, \mathrm{L}} / d t_{1 \tau, \text { Sprung }}$ & 0,298 & $5,86 \mathrm{E}+09$ & $5,22 \mathrm{E}+09$ & 9,05 & 208 & $25,3 \%$ & $\pm 1,5 \%$ \\
\hline$\Delta \vartheta_{\text {Sprung }}=10 \mathrm{~K}$ & & $6,04 \mathrm{E}+06$ & $5,20 \mathrm{E}+06$ & 10,88 & 31 & $15,8 \%$ & $\pm 1,8 \%$ \\
\hline$\Delta t_{\text {Sprung }}$ & $00: 00: 34$ & $4,87 E+07$ & $4,15 E+07$ & 11,16 & 52 & $21,2 \%$ & $\pm 2,4 \%$ \\
\hline$\Delta t_{\text {isPh }}$ & $00: 04: 32$ & $1,63 E+08$ & $1,41 E+08$ & 11,40 & 71 & $23,9 \%$ & $\pm 2,1 \%$ \\
\hline$\dot{\dot{q}}_{\text {sprung }}$ & -3 & $3,91 E+08$ & $3,34 \mathrm{E}+08$ & 11,40 & 87 & $26,4 \%$ & $\pm 1,7 \%$ \\
\hline$\overline{\dot{q}}_{\text {isPh }}$ & 35 & $7,56 \mathrm{E}+08$ & $6,54 \mathrm{E}+08$ & 11,35 & 105 & $29,1 \%$ & $\pm 1,1 \%$ \\
\hline$\dot{q}_{\max }$ & $1.393,9$ & $1,31 \mathrm{E}+09$ & $1,13 E+09$ & 11,29 & 126 & $30,2 \%$ & $\pm 0,4 \%$ \\
\hline$\Delta \vartheta_{\mathrm{L}, \min }$ & 0,0 & $2,08 E+09$ & $1,79 E+09$ & 11,22 & 148 & $29,6 \%$ & $\pm 0,7 \%$ \\
\hline$\Delta \vartheta_{L, \max }$ & 9,7 & $3,12 E+09$ & $2,67 E+09$ & 11,09 & 164 & $30,0 \%$ & $\pm 0,6 \%$ \\
\hline$\dot{q}_{\max } /\left(\Delta \vartheta_{\text {sprung }} \cdot C_{\mathrm{W}} / A_{\mathrm{W}}\right)$ & 0,040 & $4,46 \mathrm{E}+09$ & $3,80 E+09$ & 11,08 & 186 & $28,5 \%$ & $\pm 0,6 \%$ \\
\hline$d \vartheta_{\mathrm{W}, \mathrm{L}} / d t_{1 \mathrm{\tau}, \text { Sprung }}$ & 0,361 & $6,11 \mathrm{E}+09$ & $5,21 \mathrm{E}+09$ & 11,06 & 208 & $26,9 \%$ & $\pm 0,8 \%$ \\
\hline
\end{tabular}




\section{Literatur}

[Aberra et al. 2012] Aberra, T. et al.: Boundary layer instability of the natural convection flow on a uniformly heated vertical plate. In: Int. J. of Heat and Mass Transfer 55 (2012), Hefte 21 - 22, S. 6097 - 6108.

[Aydin und

Guessous 2001]

[Bejan und Lage 1990]

[Brooker et al. 2000]

[Cheeswright 1968]

[Cheeswright und Doan 1978]

[Churchill und

Chu 1975]

[Fröhlich 2006]
Aydin, O.; Guessous, L.: Fundamental correlations for laminar and turbulent free convection from a uniformly heated vertical plate. In: Int. J. of Heat and Mass Transfer 44 (2001), Heft 24, S. 4605 - 4611.

Bejan, A.; Lage, L.: The Prandtl Number Effect on the Transition in Natural Convection Along a Vertical Surface. In: J. of Heat Transfer 112 (1990), Heft 3, S. $787-790$.

Brooker, A. M. H. et al.: Convective instability in a time-dependent buoyancy driven boundary layer. In: Int. J. of Heat and Mass Transfer 43 (2000), Heft 2, S. 297 - 310.

Cheeswright, R.: Turbulent Natural Convection from a Vertical Plane Surface. In: J. of Heat Transfer 90 (1968), Heft 1, S. 1 - 6.

Cheeswright, R.; Doan, K. S.: Space-time correlation measurements in a turbulent natural convection boundary layer. In: Int. J. of Heat and Mass Transfer 21 (1978), Heft 7, S. 911 - 921.

Churchill, S. W.; Chu, H. H. S.: Correlating equations for laminar and turbulent free convection from a vertical plate. In: Int. J. of Heat and Mass Transfer 18 (1975), Heft 11, S. 1323 - 1329.

Fröhlich, J.: Large Eddy Simulation turbulenter Strömungen. 1. Aufl. Wiesbaden: B.G. Teubner, 2006. 
[Gebhart 1961]

[Gebhart 1963]

[Gebhart 1969]

[Gebhart und Adams 1963]

[Hattori et al. 2006]

[Henkes und Hoogendoorn 1990]

[Herwig und Schmandt 2015]

[Hölling 2006]

[Inagaki und Komori 1995]
Gebhart, B.: Transient Natural Convection From Vertical Elements. In: J. of Heat Transfer 83 (1961), Heft 1, S. 61 - 70.

Gebhart, B.: Transient Natural Convection From Vertical Elements - Appreciable Thermal Capacity. In: J. of Heat Transfer 85 (1963), Heft 1, S. 10 - 14.

Gebhart, B.: Natural Convection Flow, Instability, and Transition. In: J. of Heat Transfer 91 (1969), Heft 3, S. 293 - 309.

Gebhart, B.; Adams, D. E.: Measurements of Transient Natural Convection on Flat Vertical Surfaces. In: J. of Heat Transfer 85 (1963), Heft 1, S. 25 - 28.

Hattori, Y. et al.: Turbulence characteristics of natural-convection boundary layer in air along a vertical plate heated at high temperatures. In: Int. J. of Heat and Fluid Flow 27 (2006), Heft 3, S. 445 - 455.

Henkes, R. A. W. M.; Hoogendoorn, C. J.: Numerical determination of wall functions for the turbulent natural convection boundary layer. In: Int. J. of Heat and Mass Transfer 33 (1990), Heft 6, S. 1087 - 1097.

Herwig, H.; Schmandt, B.: Strömungsmechanik. 3. Aufl. Berlin, Heidelberg: Springer Verlag, 2015.

Hölling, M.: Asymptotische Analyse von turbulenten Strömungen bei hohen Rayleigh-Zahlen. Dissertation: TU Hamburg-Harburg, 2006.

Inagaki, T.; Komori, K.: Heat transfer and fluid flow of natural convection along a vertical flat plate in the transition region: experimental analysis of the wall temperature field. In: Int. J. of Heat and Mass Transfer 38 (1995), Heft 18, S. 3485 - 3495. 
[Joshi und

Gebhart 1987]

[Joshi und

Gebhart 1988]

[Kast 1972]

[Khalifa 2001]

[Kitamura et al. 1985]

[Kriegel 1973]

[Kriegel 2005]

[Lin et al. 2008]

[Lin und

Armfield 2017]
Joshi, Y.; Gebhart, B.: Transition of transient vertical natural-convection flows in water. In: J. of Fluid Mechanics 179 (1987), S. 407 - 438.

Joshi, Y.; Gebhart, B.: Transient response of a steady vertical flow subject to a change in surface heating rate. In: Int. J. of Heat and Mass Transfer 31 (1988), Heft 4, S. $743-757$.

Kast, W.: Konvektive Wärme- und Stoffübertragung in einheitlicher Betrachtung. In: Verfahrenstechnik 6 (1972), Heft 10, S. 346 - 354.

Khalifa, A.-J. N.: Natural convective heat transfer coefficient - a review: I. Isolated vertical and horizontal surfaces. In: Energy Conversion and Management 42 (2001), Heft 4, S. 491 - 504.

Kitamura, K. et al.: Large eddy structure and heat transfer of turbulent natural convection along a vertical flat plate. In: Int. J. of Heat and Mass Transfer 28 (1985), Heft 4, S. 837 - 850.

Kriegel, B.: Fallströmungen vor Abkühlflächen in Gebäuden und mögliche Schutzmaßnahmen. Dissertation: TU Berlin, 1973.

Kriegel, M.: Numerische Simulation von Quellluftsystemen. Dissertation: TU Berlin, 2005.

Lin, W. et al.: Unsteady natural convection boundary-layer flow of a linearly-stratified fluid with $\operatorname{Pr}<1$ on an evenly heated semi-infinite vertical plate. In: Int. J. of Heat and Mass Transfer 51 (2008), Hefte 1 2, S. $327-343$.

Lin, W.; Armfield, S. W.: Scalings for unsteady natural convection boundary layers on a vertical plate at time-dependent temperature. In: Int. J. of Thermal Sciences 111 (2017), S. 78 - 99. 
[Maranzana

et al. 2002]

[Martin 1984]

[Martynenko und Khramtsov 2005]

[Mollendorf und Gebhart 1970]

[Miyamoto und Okayama 1982]

[Oertel jr. 2017]

[Patterson et al. 2002] Patterson, J. C. et al.: Boundary layer development on a semi-infinite suddenly heated vertical plate. In: J. of Fluid Mechanics 453 (2002), S. 39 - 55.

[Paul und Rees 2008] Paul, M. C.; Rees, D. A. S.: Numerical Investigation of the Linear Stability of a Free Convection Boundary Layer Flow Using a Thermal Disturbance With a Slowly Increasing Frequency. In: J. of Heat Transfer 130 (2008), Heft 12, S. 122501-1 - 122501-8. 
[Pirovano et al 1970] Pirovano, A. et al.: Convection Naturelle en Regime Turbulent le long d'une Plaque Plane Verticale. In: IHTC (1970), Proceedings of the 4th Int. Heat Transfer Conference, Paris-Versaille, Beitrag NC1.8.

[Saha et al. 2012]

Saha, S. C. et al.: Prandtl number scaling of the unsteady natural convection boundary layer adjacent to a vertical flat plate for $\mathrm{Pr}>1$ subject to ramp surface heat flux. In: Int. J. of Heat and Mass Transfer 55 (2012), Heft 23/24, S. 7046 - 7055.

[Sammakia et al. 1985] Sammakia, B. et al.: Measurements and calculations of transient mixed convection in air. In: Int. J. of Heat and Mass Transfer 28 (1985), Heft 10, S. 1837 1846.

[Saunders 1936]

Saunders, O. A.: The effect of pressure upon natural convection in air. In: Proceedings of the Royal Society of London, Series A 157 (1936), Heft 891, S. 278 291.

[Schlichting und Gersten 2006]

[Schlünder 1970]

[Siebers et al. 1985]

[Tsuji und

Nagano 1988]
Schlichting, H.; Gersten, K.: Grenzschicht-Theorie. 10. Aufl. Berlin, Heidelberg: Springer-Verlag, 2006.

Schlünder, E.-U.: Über eine zusammenfassende Darstellung der Grundgesetze des konvektiven Wärmeüberganges. In: Verfahrenstechnik 4 (1970), Heft 1, S. 11 - 16.

Siebers, D. L. et al.: Experimental, Variable Properties Natural Convection From a Large, Vertical, Flat Surface. In: J. of Heat Transfer 107 (1985), Heft 1, S. $124-132$.

Tsuji, T.; Nagano, Y.: Characteristics of a turbulent natural convection boundary layer along a vertical flat plate. In: Int. J. of Heat and Mass Transfer 31 (1988), Heft 8, S. 1723 - 1734. 
[Tsuji und

Nagano 1989]

[VDI 2013]

[Vitharana und

Lykodis 1994]

[Vliet und Ross 1975]

[Wagner 2011]

[Warner und

Arpaci 1968]

[Wilcox 2002]

[Xu et al. 1998]

[Yang 2001]
Tsuji, T.; Nagano, Y.: Velocity and temperature measurements in a natural convection boundary layer along a vertical flat plate. In: Experimental Thermal and Fluid Science 2 (1989), Heft 2, S. 208 215.

VDI e.V. - Gesellschaft Verfahrenstechnik und Chemieingenieurwesen (Hrsg.): VDI-Wärmeatlas. 11. Aufl. Berlin, Heidelberg: Springer-Verlag, 2013.

Vitharana, V. L.; Lykoudis, P. S.: Criteria for Predicting the Transition to Turbulence in Natural Convection Along a Vertical Surface. In: J. of Heat Transfer 116 (1994), Heft 3, S. 633 - 638.

Vliet, G. C.; Ross, D. C.: Turbulent Natural Convection on Upward and Downward Facing Inclined Constant Heat Flux Surfaces. In: In: J. of Heat Transfer 97 (1975), Heft 4, S. 549 - 554.

Wagner, W.: Wärmeübertragung. 7. Aufl. Würzburg: Vogel Business Media, 2011.

Warner, C. Y.; Arpaci, V. S.: An experimental investigation of turbulent natural convection in air at low pressure along a vertical heated flat plate. In: Int. J. of Heat and Mass Transfer 11 (1968), Heft 3, S. 397 406.

Wilcox, D.C.: Turbulence Modeling for CFD. 2. Aufl. La Canada: DCW Industries, 2002.

$\mathrm{Xu}, \mathrm{W}$. et al.: A new turbulence model for near-wall natural convection. In: Int. J. of Heat and Mass Transfer 41 (1998), Heft 21, S. 3161 - 3176.

Yang, S.-M.: Improvement of the basic correlating equations and transition criteria of natural convection heat transfer. In: Heat Transfer - Asian Research 30 (2001), Heft 4, S. 293 - 300. 
[Zhao et al. 2013]

[Zhao et al. 2015]

[Zhao et al. 2016]

[Zhao et al. 2019]
Zhao, Y. et al.: Resonance of the thermal boundary layer adjacent to an isothermally heated vertical surface. In: J. of Fluid Mechanics 724 (2013), S. 305 336.

Zhao, Y. et al.: A PIV measurement of the natural transition of a natural convection boundary layer. In: Experiments in Fluids 56 (2015), Heft 1, Artikel 9.

Zhao, Y. et al.: Natural transition in natural convection boundary layers. In: Int. Communications in Heat and Mass Transfer 76 (2016), S. 366 - 375.

Zhao, Y. et al.: PIV measurements of the K-type transition in natural convection boundary layers. In: Experimental Thermal and Fluid Science 101 (2019), S. $62-75$. 


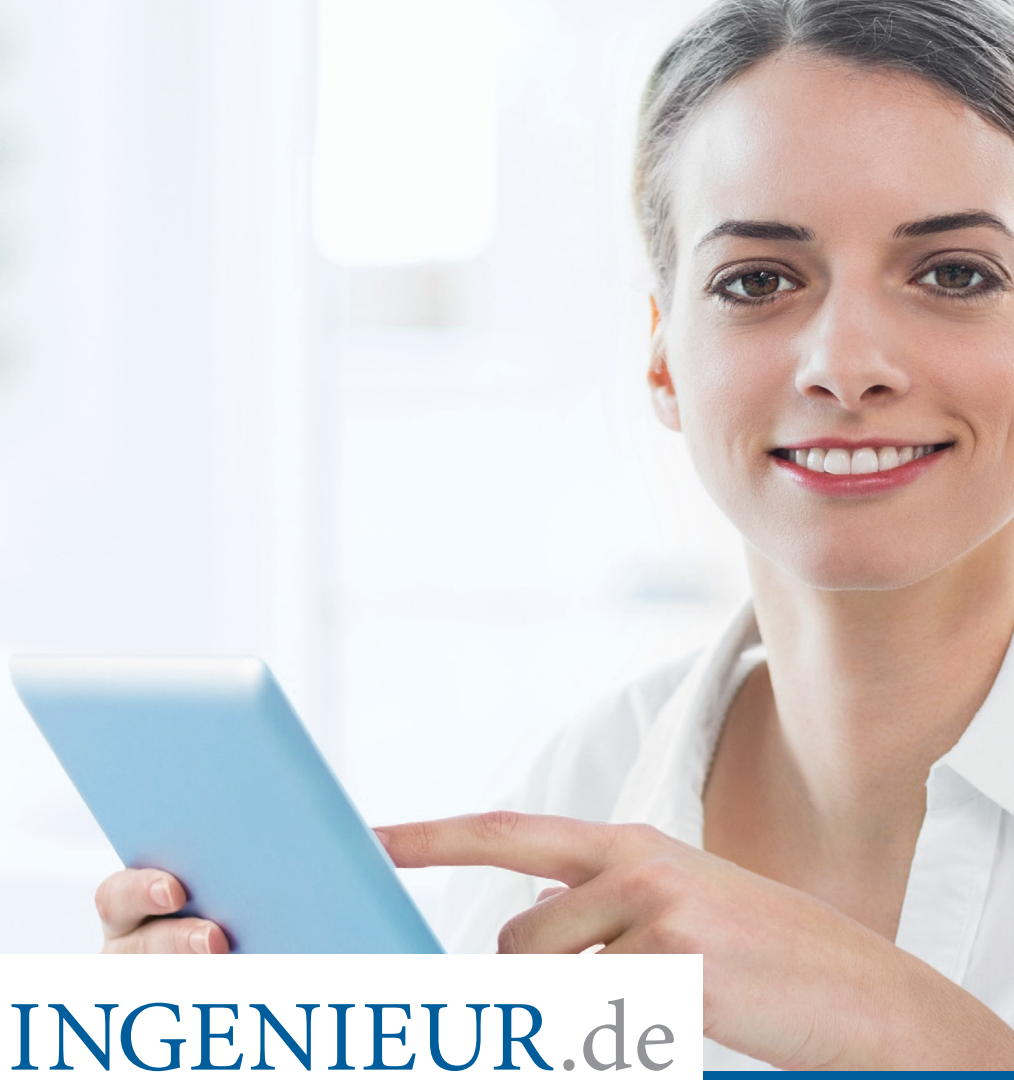

senomp

(x)
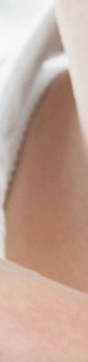


\section{Die Reihen der Fortschritt-Berichte VDI:}

1 Konstruktionstechnik/Maschinenelemente

2 Fertigungstechnik

3 Verfahrenstechnik

4 Bauingenieurwesen

5 Grund- und Werkstoffe/Kunststoffe

6 Energietechnik

7 Strömungstechnik

8 Mess-, Steuerungs- und Regelungstechnik

9 Elektronik/Mikro- und Nanotechnik

10 Informatik/Kommunikation

11 Schwingungstechnik

12 Verkehrstechnik/Fahrzeugtechnik

13 Fördertechnik/Logistik

14 Landtechnik/Lebensmitteltechnik

15 Umwelttechnik

16 Technik und Wirtschaft

17 Biotechnik/Medizintechnik

18 Mechanik/Bruchmechanik

19 Wärmetechnik/Kältetechnik

20 Rechnerunterstützte Verfahren (CAD, CAM, CAE CAQ, CIM ...)

21 Elektrotechnik

22 Mensch-Maschine-Systeme

23 Technische Gebäudeausrüstung 